\title{
Ag-Catalyzed Oxidative ipso-Cyclization via Decarboxylative Acylation/Alkylation: Access to 3-Acyl/Alkyl-spiro[4.5]trienones
}

\author{
Chada Raji Reddy, ${ }^{*,+*}$ Dattahari H. Kolgave, ${ }^{\dagger, *}$ Muppidi Subbarao, ${ }^{\dagger, *}$ Mounika Aila ${ }^{\dagger, *}$ and \\ Santosh Kumar Prajapti*,†
}

$\dagger$ Department of Organic Synthesis \& Process Chemistry, CSIR-Indian Institute of Chemical Technology, Hyderabad 500007, India. \$Academy of Scientific and Innovative Research (AcSIR), Ghaziabad 201 002, India

\section{Table of Contents}

1. General information. S1-S2

2. Optimization of alkylative ipso-cyclizatoin (Table S1)

S3

3. Experimental procedures and characterization data of compounds

4. Gram scale reaction procedure for $\mathbf{3 b}$

S30-S31

5. References

6. ${ }^{1} \mathrm{H}$ NMR and ${ }^{13} \mathrm{C}$ NMR and 19F NMR spectral copies of compounds......

7. HRMS spectral copy of $\mathrm{H}_{2}{ }^{18} \mathrm{O}$ experiment

S125-S126

8. HRMS spectral copy of TEMPO experiment

\section{General information:}

All the reactions were performed in oven-dried glass apparatus, the air and moisture sensitive reactions were carried out under inert atmosphere (nitrogen) using freshly distilled anhydrous solvents. Commercially available reagents were used as such without further purification. All reactions were monitored by thin-layer chromatography carried out on silica plates using UV-light and anisaldehyde for visualization. Column chromatography was performed on silica gel (100-200 mesh) using hexane and ethyl acetate as eluent. ${ }^{1} \mathrm{H}$ NMR was recorded in $\mathrm{CDCl}_{3}$ on $500 \mathrm{MHz}, 400$ $\mathrm{MHz}$ and $300 \mathrm{MHz}$ and ${ }^{13} \mathrm{C}$ NMR was recorded on $125 \mathrm{MHz}, 100 \mathrm{MHz}$ and $75 \mathrm{MHz}$. $\delta 7.26$ and $\delta 77$ are corresponding to $\mathrm{CDCl}_{3}$ in ${ }^{1} \mathrm{H}$ NMR and ${ }^{13} \mathrm{C}$ NMR respectively, $\delta 1.56$ is related to moisture present in $\mathrm{CDCl}_{3}$. Chemical shifts were reported in $\delta(\mathrm{ppm})$ relative to TMS as an internal standard and $J$ values were given in $\mathrm{Hz}$ (hertz). Multiplicity is indicated as, s (singlet); d (doublet); $\mathrm{t}$ (triplet); $\mathrm{m}$ (multiplet); dd (doublet of doublets), etc. FTIR spectra were recorded on Alpha (Bruker) Infrared Spectrophotometer. High resolution mass spectra (HRMS) [ESI+] were obtain using either a TOF or a double focusing spectrometer. $\alpha$-Keto acids $2 \mathbf{a}$ to $\mathbf{2 e}$ and alkyl carboxylic acids $\mathbf{4 a}-\mathbf{4 h}$ were purchased from commercial sources. 
Procedures for the Preparation of Starting Materials:

All the starting materials $(1 \mathrm{a} \text { to } \mathbf{1 f})^{\mathbf{1 a}},(\mathbf{1 h}, \mathbf{1 j}, \mathbf{1 k}, \mathbf{1 n}, \mathbf{1 p}, \mathbf{1 q}, \mathbf{1 s} \text { and } 1 \mathrm{t})^{\mathbf{1 a}},(\mathbf{1 g}, \mathbf{1 l} \text { and } 1 \mathrm{~m})^{\mathbf{1 b}}$ and $\mathbf{6 a}^{1 \mathbf{c}}$ were prepared based on literature reports and the spectral data was compared.<smiles>COc1ccc(N(C)C(=O)C#Cc2ccccc2)cc1</smiles>

$1 \mathbf{a}$<smiles>COc1ccc(N(C)C(=O)C#Cc2ccc(F)cc2)cc1</smiles>

1f

$\mathbf{1 b}$<smiles>COc1ccc(C#CC(=O)N(C)c2ccc(OC)cc2)cc1</smiles>

$1 c$<smiles>COc1ccc(C#CC(=O)N(C)c2ccc(OC)cc2)cc1</smiles>

$1 d$
1 e<smiles>COc1ccc(N(C)C(=O)C#Cc2ccc(C#N)cc2)cc1</smiles>

$1 \mathrm{~g}$<smiles>COc1ccc(N(C)C(=O)C#Cc2cccc(C(F)(F)F)c2)cc1</smiles>

$1 \mathbf{i}$<smiles>COc1ccc(N(C)C(=O)C#Cc2cccc3ccccc23)cc1</smiles>

$1 \mathbf{j}$<smiles>COc1ccc(N(C)C(=O)C#Cc2cccs2)cc1</smiles>

$1 \mathrm{k}$<smiles>C#CC(=O)N(C)c1ccc(OC)cc1</smiles>

11<smiles>CC#CC(=O)N(C)c1ccc(OC)cc1</smiles>

$1 \mathrm{~m}$<smiles>COc1ccc(N(C)C(=O)C#Cc2ccccc2)cc1C</smiles>

1 n<smiles>COc1ccc(N(C)C(=O)C#Cc2ccccc2)cc1F</smiles>

10<smiles>COc1ccc(N(C)C(=O)C#Cc2ccccc2)c(C)c1</smiles>

$1 \mathrm{p}$<smiles>COc1ccc(N(Cc2ccccc2)C(=O)C#Cc2ccccc2)cc1</smiles>

$1 q$<smiles></smiles>

1r<smiles>COc1ccc(N(C)C(=O)C#Cc2ccccc2)cc1</smiles>

$1 s$<smiles>Cc1ccc(N(Cc2ccccc2I)C(=O)C#Cc2ccccc2)cc1</smiles>

$1 t$<smiles>COc1ccc2c(c1)CCCN2C(=O)C#Cc1ccccc1</smiles>

$6 a$<smiles>COc1ccc2c(c1)CCCN2C(=O)C#Cc1ccc(Cl)cc1</smiles>

6b<smiles>COc1ccc2c(c1)CCCN2C(=O)C#Cc1ccc(I)cc1</smiles> 
Table S1: Optimization table:

Optimization of Reaction Conditions for 3-alkylspiro[4,5]trienones ${ }^{a}$<smiles>COc1ccc(N(C)C(=O)C#Cc2ccccc2)cc1</smiles>

1a

$\begin{array}{ccccc}\text { entry } & \begin{array}{c}\text { catalyst } \\ (10 \mathrm{~mol} \%)\end{array} & \begin{array}{l}\text { oxidant } \\ \text { (5 equiv) }\end{array} & \text { solvent } & \text { yield }(\%)^{\mathrm{b}} \\ 1 & \mathrm{AgNO}_{3} & \mathrm{~K}_{2} \mathrm{~S}_{2} \mathrm{O}_{8} & \mathrm{CH}_{3} \mathrm{CN}: \mathrm{H}_{2} \mathrm{O}(1: 1) & 0 \\ 2 & \mathrm{Ag}_{2} \mathrm{CO}_{3} & \mathrm{~K}_{2} \mathrm{~S}_{2} \mathrm{O}_{8} & \mathrm{CH}_{3} \mathrm{CN}: \mathrm{H}_{2} \mathrm{O}(9: 1) & 40 \\ 3 & \mathrm{Ag}_{2} \mathrm{CO}_{3} & \mathrm{~K}_{2} \mathrm{~S}_{2} \mathrm{O}_{8} & \mathrm{CH}_{3} \mathrm{CN}: \mathrm{H}_{2} \mathrm{O}(8: 2) & 55 \\ 4 & \mathrm{Ag}_{2} \mathrm{CO}_{3} & \mathrm{~K}_{2} \mathrm{~S}_{2} \mathrm{O}_{8} & \mathrm{CH}_{3} \mathrm{CN}: \mathrm{H}_{2} \mathrm{O}(1: 1) & \mathbf{7 4}\end{array}$

aUnless otherwise stated, all the reactions were performed using 0.3 $\mathrm{mmol}$ of $1 \mathrm{a}$ with $0.6 \mathrm{mmol}$ of $\mathbf{4 a}$ in $3 \mathrm{~mL}$ of solvent mixture (1:1); ${ }^{b}$ Isolated yield.

\section{Experimental procedures and characterization data of compounds:}

\section{General Procedure for the Synthesis of propiolamides:}

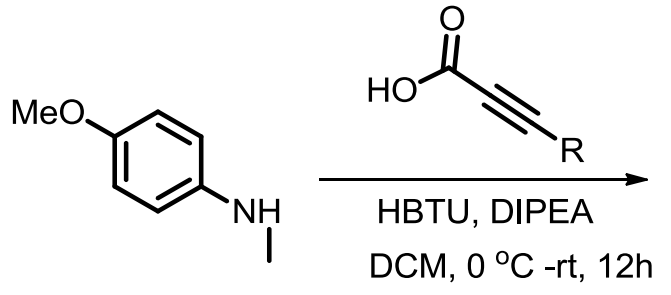<smiles>[R]C#CC(=O)N(C)c1ccc(OC)cc1</smiles>

To a stirred solution of Acid (1.1 equiv) in DCM under nitrogen condition at $0{ }^{\circ} \mathrm{C}$ then add HBTU (1.5 equiv). After 30 mins add aniline (1 equiv) followed by addition of DIPEA drop by drop period of 5 mins at $0{ }^{\circ} \mathrm{C}$. The reaction mixture was allowed to stir for $12 \mathrm{~h}$ at room temperature. The reaction quenched with water. The aqueous solution was extracted with DCM $(3 X 50 \mathrm{~mL})$. The combined organic layers were washed with brine solution $(50 \mathrm{~mL})$. After the organic layer was dried with $\mathrm{Na}_{2} \mathrm{SO}_{4}$, the solvent was removed under reduced pressure to get crude. The crude was purified by column chromatography on silica gel (100-200) using petroleum ether and EtOAc as eluent to obtain $\mathrm{N}$-aryl propiolamides. 
N-(4-Methoxyphenyl)-N-methyl-3-(3-(trifluoromethyl)phenyl)propiolamide (1i):

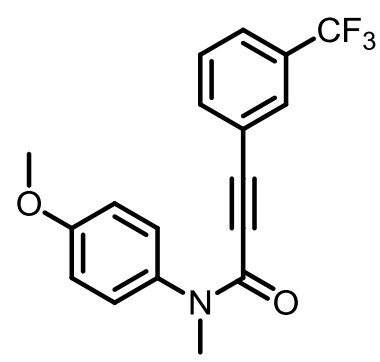

Following the general procedure, to a stirred solution of 3-(3-(trifluoromethyl)phenyl)propiolic acid (859 mg, 1.1 equiv) in DCM under nitrogen condition at $0{ }^{\circ} \mathrm{C}$ then add $\mathrm{HBTU}$ (1.3 g, 1.5 equiv). After 30 mins add 4-methoxy-N-methyl aniline (500 mg, 1 equiv) followed by addition of DIPEA( $2 \mathrm{ml}, 3$ equiv) drop by drop period of 5 mins at $0{ }^{\circ} \mathrm{C}$. The reaction mixture was allowed to stir for $12 \mathrm{~h}$ at room temperature. The reaction quenched with water. The aqueous solution was extracted with DCM ( 3 X $50 \mathrm{~mL}$ ). The combined organic layers were washed with brine solution $(50 \mathrm{~mL})$. After the organic layer was dried with $\mathrm{Na}_{2} \mathrm{SO}_{4}$, the solvent was removed under reduced pressure to get crude. The crude was purified by column chromatography on silica gel (100-200) using petroleum ether and EtOAc as eluent to obtain rotomers N-(4-methoxyphenyl)-N-methyl-3-(3(trifluoromethyl)phenyl)propiolamide 1i, $950 \mathrm{mg}$, 78\% yield, Yellow solid, mp: $45-46{ }^{\circ} \mathrm{C} ; \mathrm{R}_{f}=0.4$ ( Petroleum ether : EtOAc = 7:3); ${ }^{1} \mathrm{H}$ NMR $\left(400 \mathrm{MHz}, \mathrm{CDCl}_{3}\right) \delta 7.60-7.54(\mathrm{~m}, 1 \mathrm{H}), 7.42-7.38(\mathrm{~m}$, $2 \mathrm{H}), 7.34(\mathrm{~d}, 1 \mathrm{H}), 7.29-7.24(\mathrm{~m}, 3 \mathrm{H}), 7.03-6.92(\mathrm{~m}, 2 \mathrm{H}), 3.85$ (s, 3H), $3.36(\mathrm{~s}, 3 \mathrm{H}) ;{ }^{13} \mathrm{C}$ NMR $(101$ $\left.\mathrm{MHz}, \mathrm{CDCl}_{3}\right) \delta 159.3,154.1,135.9,135.2,131.2,129.3,129.3,128.0,128.6,126.7,126.4,126.3$, 121.5, 114.3, 88.9, 83.8, 55.5, 36.6; ${ }^{19} \mathrm{~F}$ NMR (376 $\left.\mathrm{MHz}, \mathrm{CDCl}_{3}\right) \delta-63.21$ (s, 3F); IR (KBr): vmax $=3469,2935,2221,1637,1124,696 \mathrm{~cm}^{-1}$; HRMS (ESI) $: \mathrm{m} / \mathrm{z}$ calcd for $\mathrm{C}_{18} \mathrm{H}_{14} \mathrm{~F}_{3} \mathrm{NO}_{2}(\mathrm{M}+\mathrm{H})^{+}$: 334.1055, Found: 334.1065.

N-(3-Fluoro-4-methoxyphenyl)-N-methyl-3-phenylpropiolamide (10):<smiles>COc1ccc(N(C)C(=O)C#Cc2ccccc2)cc1F</smiles>

Following the general procedure, to a stirred solution of 3-phenylpropiolic acid (518 mg, 1.1 equiv) in DCM under nitrogen condition at $0{ }^{\circ} \mathrm{C}$ then add HBTU ( $1.8 \mathrm{~g}, 1.5$ equiv). After 30 mins add 3 -fluoro4-methoxy-N-methyl aniline (500 mg, 1 equiv) followed by addition of DIPEA (1.7 ml, 3 equiv) drop by drop period of 5 mins at $0{ }^{\circ} \mathrm{C}$. The reaction mixture was allowed to stir for $12 \mathrm{~h}$ at room temperature. The reaction quenched with water. The aqueous solution was extracted with DCM (3X50 
$\mathrm{mL})$. The combined organic layers were washed with brine solution $(50 \mathrm{~mL})$. After the organic layer was dried with $\mathrm{Na}_{2} \mathrm{SO}_{4}$, the solvent was removed under reduced pressure to get crude. The crude was purified by column chromatography on silica gel (100-200) using petroleum ether and EtOAc as eluent to obtain rotomers of N-(3-fluoro-4-methoxyphenyl)-N-methyl-3-phenylpropiolamide 1o, 700 $\mathrm{mg}, 77 \%$ yield, white solid, mp: $114-116{ }^{\circ} \mathrm{C} ; \mathrm{R}_{f}=0.6$ ( Petroleum ether : EtOAc $=7: 3$ ); ${ }^{1} \mathrm{H} \mathrm{NMR}$ $\left(400 \mathrm{MHz}, \mathrm{CDCl}_{3}\right) \delta 7.38-7.32(\mathrm{~m}, 1 \mathrm{H}), 7.29-7.24(\mathrm{~m}, 2 \mathrm{H}), 7.24-7.19(\mathrm{~m}, 2 \mathrm{H}), 7.16-7.07$ (m, $2 \mathrm{H}), 7.02-6.97(\mathrm{~m}, 1 \mathrm{H}), 3.94(\mathrm{~s}, 3 \mathrm{H}), 3.35(\mathrm{~s}, 3 \mathrm{H}) ;{ }^{13} \mathrm{C} \mathrm{NMR}\left(101 \mathrm{MHz}, \mathrm{CDCl}_{3}\right) \delta 154.4,153.1$, 150.6, 147.5, 147.4, 136.0, 136.0, 132.4, 130.3, 130.0, 128.6, 128.4, 123.5, 123.4, 120.3, 115.8, 115.6, 113.2, 91.2, 82.3, 56.5, 36.5; ${ }^{19} \mathrm{~F} \mathrm{NMR}\left(376 \mathrm{MHz}, \mathrm{CDCl}_{3}\right) \delta-132.85$ (s, 1F); IR (KBr): vmax =3506, 2936, 2216, 1641,1111, $694 \mathrm{~cm}^{-1}$; HRMS (ESI) : m/z calcd for $\mathrm{C}_{17} \mathrm{H}_{14} \mathrm{FNO}_{2}(\mathrm{M}+\mathrm{H})^{+}$: 284.1087, Found: 284.1095.

\section{3-(4-Chlorophenyl)-1-(6-methoxy-3,4-dihydroquinolin-1(2H)-yl)prop-2-yn-1-one (6b):}

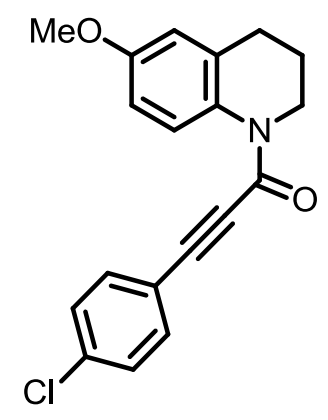

Following the general procedure, to a stirred solution of 3-(4-chlorophenyl) propiolic acid (600 mg, 1.1 equiv) in DCM under nitrogen condition at $0{ }^{\circ} \mathrm{C}$ then add HBTU (1.74 g, 1.5 equiv). After 30 mins add 6-methoxy-1,2,3,4-tetrahydroquinoline (500 mg, 1 equiv) followed by addition of DIPEA (1.65 $\mathrm{ml}, 3$ equiv) drop by drop period of 5 mins at $0{ }^{\circ} \mathrm{C}$. The reaction mixture was allowed to stir for $12 \mathrm{~h}$ at room temperature. The reaction quenched with water. The aqueous solution was extracted with DCM (3 X $50 \mathrm{~mL}$ ). The combined organic layers were washed with brine solution (50 mL). After the organic layer was dried with $\mathrm{Na}_{2} \mathrm{SO}_{4}$, the solvent was removed under reduced pressure to get crude. The crude was purified by column chromatography on silica gel (100-200) using petroleum ether and EtOAc as eluant to obtain equimolar ratio of rotomers of 3-(4-chlorophenyl)-1-(6-methoxy-3,4dihydroquinolin-1(2H)-yl)prop-2-yn-1-one $6 \mathbf{b}, 804 \mathrm{mg}, 80 \%$ yield, Yellow solid, $\mathrm{R}_{f}=0.4$ ( Petroleum ether : EtOAc = 7:3), mp: $103-105{ }^{\circ} \mathrm{C} ;{ }^{1} \mathrm{H}$ NMR $\left(400 \mathrm{MHz}, \mathrm{CDCl}_{3}\right) \delta 7.98(\mathrm{~d}, J=9.0 \mathrm{~Hz}, 1 \mathrm{H}), 7.55$ (dd, $J=21.4,8.5 \mathrm{~Hz}, 3 \mathrm{H}), 7.35$ (dd, $J=11.1,8.7 \mathrm{~Hz}, 4 \mathrm{H}), 7.29$ (d, $J=8.5 \mathrm{~Hz}, 2 \mathrm{H}), 4.18-4.03$ (m, 2H), $3.88(\mathrm{t}, J=6.5 \mathrm{~Hz}, 2 \mathrm{H}), 3.82(\mathrm{~s}, \mathrm{H}), 3.79(\mathrm{~s}, 3 \mathrm{H}), 2.87(\mathrm{t}, J=6.7 \mathrm{~Hz}, 2 \mathrm{H}), 2.76(\mathrm{t}, J=6.5 \mathrm{~Hz}$, $2 \mathrm{H}), 2.13-2.03(\mathrm{~m}, 2 \mathrm{H}), 2.03-1.95(\mathrm{~m}, 2 \mathrm{H}) ;{ }^{13} \mathrm{C} \mathrm{NMR}\left(101 \mathrm{MHz}, \mathrm{CDCl}_{3}\right) \delta$ 157.6, 156.6, 153.1, 152.8, 136.3, 134.4, 133.6, 130.9, 130.9, 130.2, 129.0, 128.9, 125.6, 125.5, 119.1, 113.7, 113.0, 111.7, $111.3,90.1,88.6,84.2,83.3,55.5,55.4,47.5,42.8,27.2,27.2,23.6,23.6$; IR $(\mathrm{KBr}): v \max =3906$, 
3614, 3574, 1631, 1562, $775 \mathrm{~cm}^{-1}$; HRMS (ESI): $\mathrm{m} / \mathrm{z}$ calcd for $\mathrm{C}_{19} \mathrm{H}_{17} \mathrm{NO}_{2} \mathrm{Cl}(\mathrm{M}+\mathrm{H})^{+}: 326.0948$, Found: 326.0950.

\section{3-(4-Acetylphenyl)-1-(6-methoxy-3,4-dihydroquinolin-1(2H)-yl)prop-2-yn-1-one (6c):}

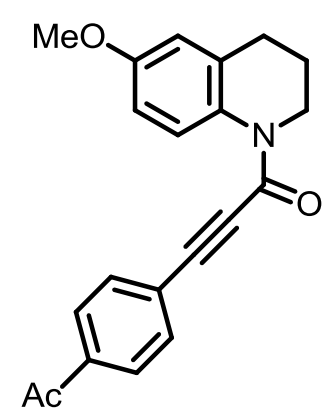

Following the general procedure, to a stirred solution of 3-(4-acetylphenyl)propiolic acid (600 mg, 1.1 equiv) in DCM under nitrogen condition at $0{ }^{\circ} \mathrm{C}$ then add HBTU (1.74 g, 1.5 equiv). After 30 mins add 6-methoxy-1,2,3,4-tetrahydroquinoline ( $500 \mathrm{mg}$, 1 equiv) followed by addition of DIPEA (1.65 $\mathrm{ml}, 3$ equiv). drop by drop period of $5 \mathrm{mins}$ at $0{ }^{\circ} \mathrm{C}$. The reaction mixture was allowed to stir for $12 \mathrm{~h}$ at room temperature. The reaction quenched with water. The aqueous solution was extracted with DCM ( 3 X $50 \mathrm{~mL}$ ). The combined organic layers were washed with brine solution (50 mL). After the organic layer was dried with $\mathrm{Na}_{2} \mathrm{SO}_{4}$, the solvent was removed under reduced pressure to get crude. The crude was purified by column chromatography on silica gel (100-200) using petroleum ether and EtOAc as eluent to obtain rotomers of 3-(4-acetylphenyl)-1-(6-methoxy-3,4-dihydroquinolin-1(2H)yl)prop-2-yn-1-one 6c, $800 \mathrm{mg}$, 78\% yield, Yellow solid, $\mathrm{R}_{f}=0.4$ ( Petroleum ether : EtOAc = 7:3), mp: $103-105{ }^{\circ} \mathrm{C} ;{ }^{1} \mathrm{H}$ NMR $\left(400 \mathrm{MHz}, \mathrm{CDCl}_{3}\right) \delta 8.05-7.86(\mathrm{~m}, 5 \mathrm{H}), 7.67(\mathrm{~d}, J=8.1 \mathrm{~Hz}, 2 \mathrm{H}), 7.58(\mathrm{~d}$, $J=8.7 \mathrm{~Hz}, 1 \mathrm{H}), 7.49(\mathrm{~d}, J=8.2 \mathrm{~Hz}, 2 \mathrm{H}), 7.42(\mathrm{~d}, J=2.9 \mathrm{~Hz}, 1 \mathrm{H}), 6.78(\mathrm{dd}, J=8.9,2.8 \mathrm{~Hz}, 2 \mathrm{H}), 6.71$ (d, $J=24.9 \mathrm{~Hz}, 2 \mathrm{H}), 4.14-4.09(\mathrm{~m}, 2 \mathrm{H}), 3.90(\mathrm{t}, J=6.5 \mathrm{~Hz}, 2 \mathrm{H}), 3.82(\mathrm{~s}, 3 \mathrm{H}), 3.79(\mathrm{~s}, 3 \mathrm{H}), 2.88(\mathrm{t}, J$ $=6.7 \mathrm{~Hz}, 2 \mathrm{H}), 2.77(\mathrm{t}, J=6.5 \mathrm{~Hz}, 2 \mathrm{H}), 2.63(\mathrm{~s}, 3 \mathrm{H}), 2.59(\mathrm{~s}, 3 \mathrm{H}), 2.17-1.93(\mathrm{~m}, 4 \mathrm{H}) ;{ }^{13} \mathrm{C} \mathrm{NMR}(101$ $\left.\mathrm{MHz}, \mathrm{CDCl}_{3}\right) \delta 197.2,157.7,156.7,152.9,152.6,137.6,137.5,134.5,132.5,131.0,130.7,130.1$, $128.3,128.2,125.6,125.5,125.3,113.8,113.1,111.8,111.3,90.1,88.6,85.7,84.8,55.5,55.4,47.5$, 42.9, 27.2, 27.1, 26.7, 23.6, 23.5; IR (KBr): $v \max =3353,2944,2832,1681,1560,778 \mathrm{~cm}^{-1}$; HRMS (ESI) : $\mathrm{m} / \mathrm{z}$ calcd for $\mathrm{C}_{21} \mathrm{H}_{20} \mathrm{NO}_{3}(\mathrm{M}+\mathrm{H})^{+}: 334.1443$, Found: 334.1446 .

\section{Synthesis of N-(4-methoxyphenyl)-3-phenyl-N-tosylpropiolamide (1r):}
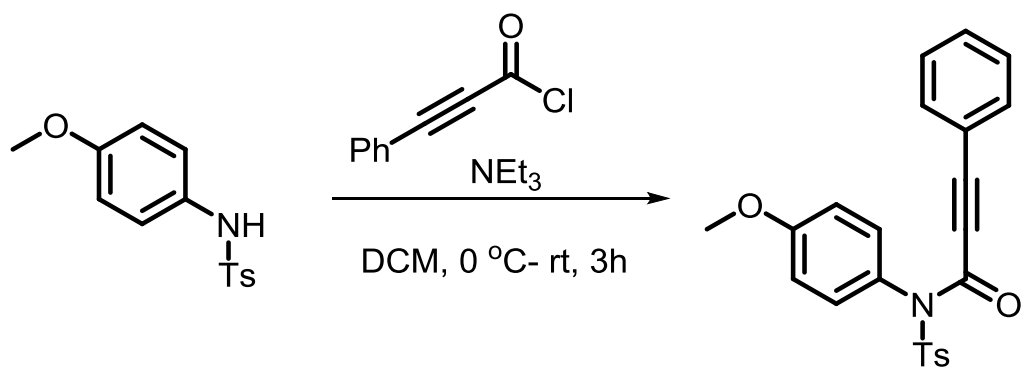
To a stirred solution of Tosyl amine ( $500 \mathrm{mg}, 1$ equiv) in DCM under nitrogen condition at $0{ }^{\circ} \mathrm{C}$ then add 3-phenylpropioloyl chloride (444 mg, 1.5 equiv), after 10 mins add Triethyl amine $(0.5 \mathrm{ml}$, 2equiv) at $0{ }^{\circ} \mathrm{C}$. The reaction mixture was allowed to stir for $3 \mathrm{~h}$ at room temperature. The reaction quenched with water and extracted with DCM $(3 X 50 \mathrm{~mL})$. The combined organic layers were washed with brine solution $(50 \mathrm{~mL})$. After the organic layer was dried with $\mathrm{Na}_{2} \mathrm{SO}_{4}$, the solvent was removed under reduced pressure to get crude. The crude was purified by column chromatography on silica gel (100- 200) using petroleum ether and EtOAc as eluent to obtain N-(4-methoxyphenyl)-3-phenyl-Ntosylpropiolamide, $600 \mathrm{mg}, 82 \%$ yield, white solid, mp: $85-87^{\circ} \mathrm{C} ; \mathrm{R}_{f}=0.4$ (Petroleum ether : EtOAc = 8:2); ${ }^{1} \mathrm{H} \mathrm{NMR}\left(400 \mathrm{MHz}, \mathrm{CDCl}_{3}\right){ }^{1} \mathrm{H}$ NMR $\left(400 \mathrm{MHz}, \mathrm{CDCl}_{3}\right) \delta 7.97(\mathrm{~d}, J=8.4 \mathrm{~Hz}, 2 \mathrm{H}), 7.39-$ $7.33(\mathrm{~m}, 3 \mathrm{H}), 7.31-7.22(\mathrm{~m}, 4 \mathrm{H}), 7.09$ (dd, $J=8.3,1.2 \mathrm{~Hz}, 2 \mathrm{H}), 7.02-6.98$ (m, 2H), $3.88(\mathrm{~s}, 3 \mathrm{H})$, 2.47 (s, 3H); ${ }^{13} \mathrm{C}$ NMR $\left(101 \mathrm{MHz}, \mathrm{CDCl}_{3}\right) \delta 160.8,152.8,145.3,135.6,132.9,131.7,130.8,129.5$, 129.2, 128.6, 128.4, 119.3, 114.6, 94.4, 81.9, 55.6, 21.7; IR (KBr): $v \max =3745,3307,2923,2212$, 1511, 1168, $766 \mathrm{~cm}^{-1}$; HRMS (ESI) : m/z calcd for $\mathrm{C}_{23} \mathrm{H}_{19} \mathrm{NO}_{4} \mathrm{~S}(\mathrm{M}+\mathrm{H})^{+}:$406.1113, Found: 406.1116.

\section{B. General procedure for synthesis of 3-acyl $[4,5]$ spirotrienones:}

\section{General reaction:}<smiles>[R]C#CC(=O)N(C)c1ccc(OC)cc1</smiles>

$$
\begin{gathered}
\begin{array}{c}
\text { Pyruvic acid (2 equiv) } \\
\mathrm{K}_{2} \mathrm{~S}_{2} \mathrm{O}_{8}(5 \text { equiv) }
\end{array} \\
\underset{\mathrm{Ag}_{2} \mathrm{CO}_{3}(10 \mathrm{~mol} \%)}{\text { Acetonitrile:water }}
\end{gathered}
$$

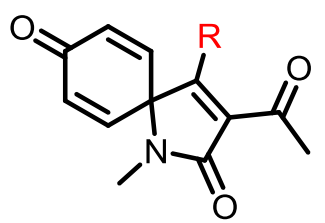

(9:1), $70^{\circ} \mathrm{C}, 6-9 \mathrm{~h}$

To a stirred solution of N-(4-methoxyaryl)-propiolamide $\mathbf{1}(0.3 \mathrm{mmol})$, pyruvic acid $\mathbf{2}(0.6 \mathrm{mmol})$ and $\mathrm{K}_{2} \mathrm{~S}_{2} \mathrm{O}_{8}(1.5 \mathrm{mmol})$ in $5 \mathrm{~mL}$ of Acetonitrile: water (9:1) in a reaction vial. Then, silver carbonate (10 mol \%) was added at room temperature. The reaction mixture stirred at $70{ }^{\circ} \mathrm{C}$ (oil bath temperature) for 6-9 h. After completion of the reaction (monitored by TLC) the mixture was concentrated under reduced pressure. The crude product was purified by column chromatography on silica gel by using EtOAc: hexanes as an eluent to afford the corresponding 3-acyl[4,5] spirotrienones. 


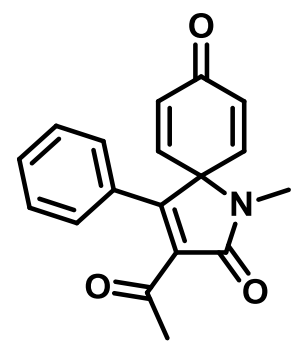

Following the general procedure, to the solution of $\mathrm{N}$-(4-methoxyphenyl)-N-methyl-3phenylpropiolamide 1a (95 mg, $0.3 \mathrm{mmol}$ ), Pyruvic acid 2a ( $53 \mathrm{mg}, 0.6 \mathrm{mmol}$ ) and $\mathrm{K}_{2} \mathrm{~S}_{2} \mathrm{O}_{8}(482 \mathrm{mg}$, $1.5 \mathrm{mmol})$ in $5 \mathrm{~mL}$ of Acetonitrile: water (9:1), silver carbonate $(8.3 \mathrm{mg}, 10 \mathrm{~mol} \%)$ was added at room temperature. The reaction mixture was stirred at $70{ }^{\circ} \mathrm{C}$ for $8 \mathrm{~h}$. After completion of the reaction (monitored by TLC), the reaction mixture was concentrated under reduced pressure. The crude product was purified by column chromatography on silica gel (EtOAc-hexanes) to afford the corresponding product 3-acetyl-1-methyl-4-phenyl-1-azaspiro[4.5]deca-3,6,9-triene-2,8-dione (3a), $98 \mathrm{mg}$, 94\% yield, off-white solid; $R_{f}=0.4$ (hexanes: EtOAc $\left.=9: 1\right)$; mp: $101-103{ }^{\circ} \mathrm{C} ;{ }^{1} \mathrm{H}$ NMR $(500$ $\left.\mathrm{MHz}, \mathrm{CDCl}_{3}\right) \delta 7.43-7.36(\mathrm{~m}, 1 \mathrm{H}), 7.37-7.30(\mathrm{~m}, 2 \mathrm{H}), 7.19(\mathrm{ddd}, J=7.5,3.3,1.8 \mathrm{~Hz}, 2 \mathrm{H}), 6.56-$ 6.46 (m, 4H), 2.90 (s, 3H), 2.43 (s, 3H); $\left.{ }^{13} \mathrm{C} \mathrm{NMR} \mathrm{(101} \mathrm{MHz,} \mathrm{CDCl}_{3}\right) \delta$ 196.0, 183.6, 167.0, 158.0, 143.8, 135.7, 133.7, 130.4, 130.2, 128.6, 127.8, 67.4, 30.8, 26.0; IR (KBr): vmax = 3520, 3054, 1702, 1632, 1380, 1223, $767 \mathrm{~cm}^{-1}$; HRMS (ESI): $\mathrm{m} / z$ calcd for $\mathrm{C}_{18} \mathrm{H}_{16} \mathrm{NO}_{3}(\mathrm{M}+\mathrm{H})^{+}:$294.1130, found: 294.1130.

\section{3-Acetyl-4-(3,5-dimethylphenyl)-1-methyl-1-azaspiro[4.5]deca-3,6,9-triene-2,8-dione (3b):}

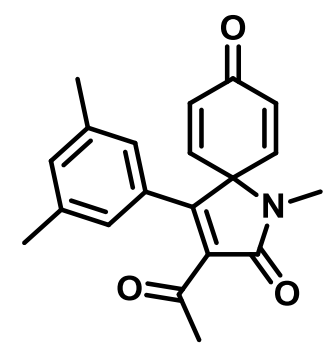

Following the general procedure, to the solution of 3-(3,5-dimethylphenyl)-N-(4-methoxyphenyl)-Nmethylpropiolamide $\mathbf{1 b}$ (90 mg, $0.3 \mathrm{mmol}$ ), Pyruvic acid $\mathbf{2 a}$ ( $53 \mathrm{mg}, 0.6 \mathrm{mmol}$ ) and $\mathrm{K}_{2} \mathrm{~S}_{2} \mathrm{O}_{8}$ ( $413 \mathrm{mg}$, $1.5 \mathrm{mmol}$ ) in $5 \mathrm{~mL}$ of Acetonitrile: water (9:1), silver carbonate $(8.3 \mathrm{mg}, 10 \mathrm{~mol} \%)$ was added at room temperature. The reaction mixture was stirred at $70{ }^{\circ} \mathrm{C}$ for $7 \mathrm{~h}$. After completion of the reaction (monitored by TLC), the reaction mixture was concentrated under reduced pressure. The crude product was purified by column chromatography on silica gel (EtOAc-hexanes) to afford the corresponding product 3-acetyl-4-(3,5-dimethylphenyl)-1-methyl-1-azaspiro[4.5]deca-3,6,9-triene2,8-dione 3b, 83 mg, 83\% yield, off-white solid; $R_{f}=0.4$ (hexanes: EtOAc $=1: 1$ ); mp: $160-162{ }^{\circ} \mathrm{C} ;{ }^{1} \mathrm{H}$ 
NMR (400 MHz, $\left.\mathrm{CDCl}_{3}\right) \delta 7.01(\mathrm{~s}, 1 \mathrm{H}), 6.75(\mathrm{~s}, 2 \mathrm{H}), 6.53-6.45(\mathrm{~m}, 4 \mathrm{H}), 2.89(\mathrm{~s}, 3 \mathrm{H}), 2.39(\mathrm{~s}, 3 \mathrm{H})$, $2.26(\mathrm{~s}, 6 \mathrm{H}) ;{ }^{13} \mathrm{C}$ NMR $\left(101 \mathrm{MHz}, \mathrm{CDCl}_{3}\right) \delta$ 196.2, 183.7, 167.1, 158.4, 144.0, 138.3, 135.5, 133.6, 132.2, 130.1, 125.3, 67.4, 30.8, 26.0, 21.3; IR (KBr): vmax = 3021, 2357, 1703, 1380, 1215, 739, 670 $\mathrm{cm}^{-1}$; HRMS (ESI): $\mathrm{m} / z$ calcd for $\mathrm{C}_{20} \mathrm{H}_{20} \mathrm{NO}_{3}(\mathrm{M}+\mathrm{H})^{+}: 322.1443$, found: 322.1453 .

\section{3-Acetyl-4-(4-methoxyphenyl)-1-methyl-1-azaspiro[4.5]deca-3,6,9-triene-2,8-dione (3c):}

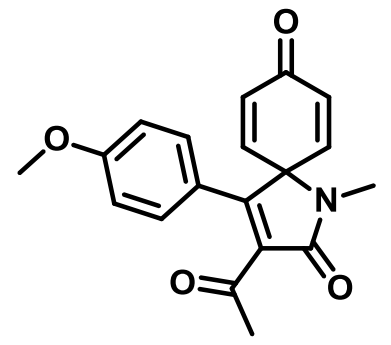

Following the general procedure, to the solution of N,3-bis(4-methoxyphenyl)-N-methylpropiolamide 1c (90 mg, $0.3 \mathrm{mmol}$ ), pyruvic acid $2 \mathrm{a}$ ( $53 \mathrm{mg}, 0.6 \mathrm{mmol}$ ) and $\mathrm{K}_{2} \mathrm{~S}_{2} \mathrm{O}_{8}(410 \mathrm{mg}, 1.5 \mathrm{mmol}$ ) in $5 \mathrm{~mL}$ of Acetonitrile: water $(9: 1)$, silver carbonate $(8.3 \mathrm{mg}, 10 \mathrm{~mol} \%)$ was added at room temperature. The reaction mixture was stirred at $70{ }^{\circ} \mathrm{C}$ for $6 \mathrm{~h}$. After completion of the reaction (monitored by TLC), the reaction mixture was concentrated under reduced pressure. The crude product was purified by column chromatography on silica gel (EtOAc-hexanes) to afford the corresponding product 3-acetyl-4-(4(methoxyphenyl)-1-methyl-azaspiro[4.5]deca-3,6,9-triene-2,8-dione 3c, $87 \mathrm{mg}, 88 \%$ yield, off-white solid; $R_{f}=0.4$ (hexanes: EtOAc $\left.=9: 1\right) ; \mathrm{mp}: 184-186{ }^{\circ} \mathrm{C} ;{ }^{1} \mathrm{H} \mathrm{NMR}\left(400 \mathrm{MHz}, \mathrm{CDCl}_{3}\right) \delta 7.16-7.11$ (m, 2H), $6.79-6.74(\mathrm{~m}, 2 \mathrm{H}), 6.47-6.43(\mathrm{~m}, J=4.2 \mathrm{~Hz}, 4 \mathrm{H}), 3.73(\mathrm{~s}, 3 \mathrm{H}), 2.81(\mathrm{~s}, 3 \mathrm{H}), 2.38$ (s, 3H); ${ }^{13} \mathrm{C}$ NMR (101 MHz, $\left.\mathrm{CDCl}_{3}\right) \delta 196.8,183.8,167.3,161.4,157.3,144.4,134.6,133.5,129.5,122.3$, 114.2, 67.1, 55.3, 30.8, 25.9; IR (KBr): vmax $=2922,2853,1685,1612,1374,763 \mathrm{~cm}^{-1}$; HRMS (ESI): $m / z$ calcd for $\mathrm{C}_{19} \mathrm{H}_{18} \mathrm{NO}_{4}(\mathrm{M}+\mathrm{H})^{+}: 324.1236$, found: 324.1232 .

3-Acetyl-1-methyl-4-(p-tolyl)-1-azaspiro[4.5]deca-3,6,9-triene-2,8-dione (3d):

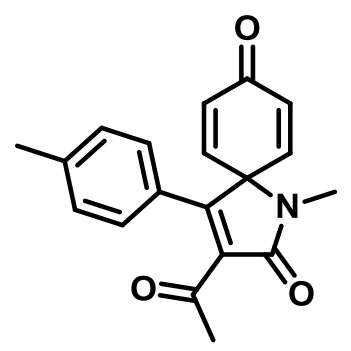

Following the general procedure, to the solution of $\mathrm{N}$-(4-methoxyphenyl)-N-methyl-3-(ptolyl)propiolamide 1d ( $85 \mathrm{mg}, 0.3 \mathrm{mmol}$ ), Pyruvic acid 2a ( $53.6 \mathrm{mg}, 0.6 \mathrm{mmol}$ ) and $\mathrm{K}_{2} \mathrm{~S}_{2} \mathrm{O}_{8}(409 \mathrm{mg}, 1.5$ mmol) in $5 \mathrm{~mL}$ of Acetonitrile: water (9:1), silver carbonate $(8.3 \mathrm{mg}, 10 \mathrm{~mol} \%)$ was added at room temperature. The reaction mixture was stirred at $70{ }^{\circ} \mathrm{C}$ for $8 \mathrm{~h}$. After completion of the reaction (monitored by TLC), the reaction mixture was concentrated under reduced pressure. The crude product was purified by column chromatography on silica gel (EtOAc-hexanes) to afford the 
corresponding product 3-acetyl-1-methyl-4-(p-tolyl)-1-azaspiro[4.5]deca-3,6,9-triene-2,8-dione 3d, $80 \mathrm{mg}, 86 \%$ yield, off-white solid; $R_{f}=0.4$ (hexanes: EtOAc $\left.=1: 1\right) ; \mathrm{mp} 142-146{ }^{\circ} \mathrm{C} ;{ }^{1} \mathrm{H}$ NMR $(400$ $\left.\mathrm{MHz}, \mathrm{CDCl}_{3}\right) \delta 7.10-6.99(\mathrm{~m}, 4 \mathrm{H}), 6.50-6.35(\mathrm{~m}, 4 \mathrm{H}), 2.81(\mathrm{~s}, 3 \mathrm{H}), 2.35(\mathrm{~s}, 3 \mathrm{H}), 2.27(\mathrm{~s}, 3 \mathrm{H}) ;{ }^{13} \mathrm{C}$ NMR $\left(101 \mathrm{MHz}, \mathrm{CDCl}_{3}\right) \delta 196.4,183.7,167.1,158.0,144.1,140.9,135.3,133.6,129.4,127.7$, 127.2, 67.3, 30.8, 25.9, 21.4; IR (KBr): vmax = 3016, 2928, 1675, 1626, 1374, 829, $755 \mathrm{~cm}^{-1}$; HRMS (ESI): $\mathrm{m} / z$ calcd for $\mathrm{C}_{19} \mathrm{H}_{17} \mathrm{NO}_{3} \mathrm{Na}(\mathrm{M}+\mathrm{Na})^{+}: 330.1106$, found: 330.1112 .

3-Acetyl-4-(4-chlorophenyl)-1-methyl-1-azaspiro[4.5]deca-3,6,9-triene-2,8-dione (3e):

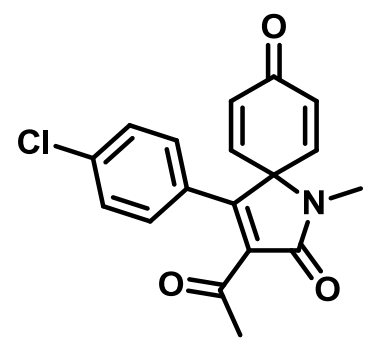

Following the general procedure, to the solution of 3-(4-chlorophenyl)-N-(4-methoxyphenyl)-Nmethylpropiolamide 1e (90 mg, $0.3 \mathrm{mmol}$ ), Pyruvic acid 2a ( $53 \mathrm{mg}, 0.6 \mathrm{mmol}$ ) and $\mathrm{K}_{2} \mathrm{~S}_{2} \mathrm{O}_{8}(404 \mathrm{mg}$, $1.5 \mathrm{mmol})$ in $5 \mathrm{~mL}$ of Acetonitrile: water (9:1), silver carbonate $(8.3 \mathrm{mg}, 10 \mathrm{~mol} \%)$ was added at room temperature. The reaction mixture was stirred at $70{ }^{\circ} \mathrm{C}$ for $9 \mathrm{~h}$. After completion of the reaction (monitored by TLC), the reaction mixture was concentrated under reduced pressure. The crude product was purified by column chromatography on silica gel (EtOAc-hexanes) to afford the corresponding product 3-acetyl-4-(4-chlorophenyl)-1-methyl-1-azaspiro[4.5]deca-3,6,9-triene-2,8dione 3e, $84 \mathrm{mg}, 85 \%$ yield, off-white solid; $R_{f}=0.4$ (hexanes: EtOAc $=1: 1$ ); mp 94-96 ${ }^{\circ} \mathrm{C} ;{ }^{1} \mathrm{H}$ NMR $\left(400 \mathrm{MHz}, \mathrm{CDCl}_{3}\right) \delta 7.36-7.34(\mathrm{~m}, 1 \mathrm{H}), 7.33-7.31(\mathrm{~m}, 1 \mathrm{H}), 7.18-7.16(\mathrm{~m}, 1 \mathrm{H}), 7.16-7.13(\mathrm{~m}$, $1 \mathrm{H}), 6.58-6.45(\mathrm{~m}, 4 \mathrm{H}), 2.92(\mathrm{~s}, 3 \mathrm{H}), 2.51(\mathrm{~s}, 3 \mathrm{H}) ;{ }^{13} \mathrm{C} \mathrm{NMR}\left(101 \mathrm{MHz}, \mathrm{CDCl}_{3}\right) \delta 195.7,183.3$, 166.9, 157.1, 143.5, 136.7, 135.9, 134.0, 129.2, 128.9, 128.5, 67.4, 30.8, 26.1; IR (KBr): vmax = 3686, 3021, 2362, 1702, 1215, 739, $670 \mathrm{~cm}^{-1}$; HRMS (ESI): $\mathrm{m} / z$ calcd for $\mathrm{C}_{18} \mathrm{H}_{15} \mathrm{NO}_{3} \mathrm{Cl}(\mathrm{M}+\mathrm{H})^{+}$: 328.0740, found: 328.0749 .

3-Acetyl-4-(4-fluorophenyl)-1-methyl-1-azaspiro[4.5]deca-3,6,9-triene-2,8-dione (3f):

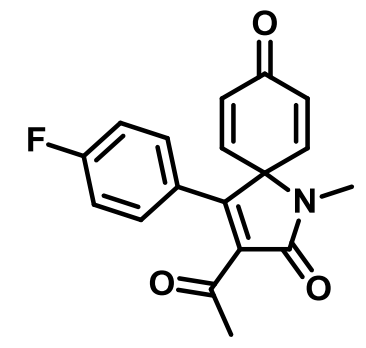

Following the general procedure, to the solution of 3-(4-fluorophenyl)-N-(4-methoxyphenyl)- $\mathrm{N}$ methylpropiolamide 1f ( $85 \mathrm{mg}, 0.3 \mathrm{mmol}$ ), Pyruvic acid 2a ( $53 \mathrm{mg}, 0.6 \mathrm{mmol}$ ) and $\mathrm{K}_{2} \mathrm{~S}_{2} \mathrm{O}_{8}$ ( $403 \mathrm{mg}$, $1.5 \mathrm{mmol}$ ) in $5 \mathrm{~mL}$ of Acetonitrile: water (9:1), silver carbonate ( $8.3 \mathrm{mg}, 10 \mathrm{~mol} \%$ ) was added at room 
temperature. The reaction mixture was stirred at $70{ }^{\circ} \mathrm{C}$ for $8 \mathrm{~h}$. After completion of the reaction (monitored by TLC), the reaction mixture was concentrated under reduced pressure. The crude product was purified by column chromatography on silica gel (EtOAc-hexanes) to afford the corresponding product 3-acetyl-4-(4-fluorophenyl)-1-methyl-1-azaspiro[4.5]deca-3,6,9-triene-2,8dione 3f, $81 \mathrm{mg}, 87 \%$ yield, off-white solid; $R_{f}=0.4$ (hexanes: EtOAc $=1: 1$ ); mp: $176-178{ }^{\circ} \mathrm{C} ;{ }^{1} \mathrm{H}$ NMR (500 MHz, $\left.\mathrm{CDCl}_{3}\right) \delta 7.21(\mathrm{dd}, J=8.6,5.2 \mathrm{~Hz}, 2 \mathrm{H}), 7.03(\mathrm{t}, J=8.5 \mathrm{~Hz}, 2 \mathrm{H}), 6.51(\mathrm{~s}, 4 \mathrm{H}), 2.90$ (s, 3H), 2.48 (s, 3H); ${ }^{13} \mathrm{C}$ NMR (101 MHz, $\left.\mathrm{CDCl}_{3}\right) \delta 195.9,183.4,166.9,163.72(\mathrm{~d}, \mathrm{~J}=252.1 \mathrm{~Hz})$, 157.1, 143.7, 135.8, 133.9, 130.04 (d, J = 8.5 Hz), 126.1, 115.90 (d, J = 21.9 Hz), 67.4, 30.8, 26.1; ${ }^{19} \mathrm{~F}$ NMR (376 MHz, $\left.\mathrm{CDCl}_{3}\right) \delta$-109.02 (s, 1F); IR (KBr): vmax = 3017, 2923, 2856, 1586, 1691, 1372, $745 \mathrm{~cm}^{-1}$; HRMS (ESI): $\mathrm{m} / z$ calcd for $\mathrm{C}_{18} \mathrm{H}_{14} \mathrm{NO}_{3} \mathrm{NaF}(\mathrm{M}+\mathrm{Na})^{+}: 334.0855$, found: 334.0859 .

\section{4-(3-Acetyl-1-methyl-2,8-dioxo-1-azaspiro[4.5]deca-3,6,9-trien-4-yl)benzonitrile (3g):}

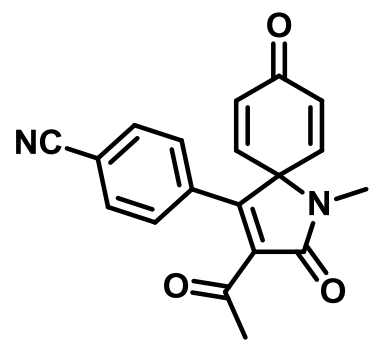

Following the general procedure, to the solution of 3-(4-cyanophenyl)-N-(4-methoxyphenyl)-Nmethylpropiolamide $1 \mathrm{~g}$ ( $87 \mathrm{mg}, 0.3 \mathrm{mmol}$ ), Pyruvic acid $\mathbf{2 a}$ ( $53 \mathrm{mg}, 0.6 \mathrm{mmol}$ ) and $\mathrm{K}_{2} \mathrm{~S}_{2} \mathrm{O}_{8}(403 \mathrm{mg}, 1.5$ $\mathrm{mmol})$ in $5 \mathrm{~mL}$ of Acetonitrile: water (9:1), silver carbonate $(8 \mathrm{mg}, 10 \mathrm{~mol} \%)$ was added at room temperature. The reaction mixture was stirred at $70{ }^{\circ} \mathrm{C}$ for $9 \mathrm{~h}$. After completion of the reaction (monitored by TLC), the reaction mixture was concentrated under reduced pressure. The crude product was purified by column chromatography on silica gel (EtOAc-hexanes) to afford the corresponding product 4-(3-acetyl-1-methyl-2,8-dioxo-1-azaspiro[4.5]deca-3,6,9-trien-4yl)benzonitrile 3g, $88 \mathrm{mg}$, 92\% yield, off-white solid; $R_{f}=0.4$ (hexanes: EtOAc $=1: 1$ ); $\mathrm{mp} 173-175{ }^{\circ} \mathrm{C}$; ${ }^{1} \mathrm{H}$ NMR (400 MHz, $\left.\mathrm{CDCl}_{3}\right) \delta 7.66-7.64(\mathrm{~m}, J=1.9 \mathrm{~Hz}, 1 \mathrm{H}), 7.63-7.61(\mathrm{~m}, 1 \mathrm{H}), 7.30-7.28(\mathrm{~m}$, $1 \mathrm{H}), 7.27-7.26(\mathrm{~m}, 1 \mathrm{H}), 6.50(\mathrm{~s}, 4 \mathrm{H}), 2.93(\mathrm{~s}, 3 \mathrm{H}), 2.55(\mathrm{~s}, 3 \mathrm{H}) ;{ }^{13} \mathrm{C} \mathrm{NMR}\left(101 \mathrm{MHz}, \mathrm{CDCl}_{3}\right) \delta$ 194.9, 182.9, 166.5, 157.0, 142.8, 136.3, 134.8, 134.3, 132.1, 128.7, 117.8, 114.0, 67.4, 30.6, 26.2; IR $(\mathrm{KBr}): \quad v \max =3018,2230,1681,1372,849,753 \mathrm{~cm}^{-1}$; HRMS (ESI): $\mathrm{m} / z$ calcd for $\mathrm{C}_{19} \mathrm{H}_{15} \mathrm{~N}_{2} \mathrm{O}_{3}(\mathrm{M}+\mathrm{H})^{+}: 319.1083$, found: 319.1088 .

3-Acetyl-4-(4-acetylphenyl)-1-methyl-1-azaspiro[4.5]deca-3,6,9-triene-2,8-dione (3h):<smiles>CC(=O)C1=C(c2ccc(C(C)=O)cc2)C2(C=CC(=O)C=C2)N(C)C1=O</smiles> 
Following the general procedure, to the solution of 3-(4-acetylphenyl)-N-(4-methoxyphenyl)-Nmethylpropiolamide $\mathbf{1 h}$ (93 mg, $0.3 \mathrm{mmol}$ ), Pyruvic acid $\mathbf{2 a}$ ( $53.3 \mathrm{mg}, 0.6 \mathrm{mmol}$ ) and $\mathrm{K}_{2} \mathrm{~S}_{2} \mathrm{O}_{8}(407 \mathrm{mg}$, $1.5 \mathrm{mmol}$ ) in $5 \mathrm{~mL}$ of Acetonitrile: water (9:1), silver carbonate (8.3 $\mathrm{mg}, 10 \mathrm{~mol} \%$ ) was added at room temperature. The reaction mixture was stirred at $70{ }^{\circ} \mathrm{C}$ for $8 \mathrm{~h}$. After completion of the reaction (monitored by TLC), the reaction mixture was concentrated under reduced pressure. The crude product was purified by column chromatography on silica gel (EtOAc-hexanes) to afford the corresponding product 3-acetyl-4-(4-acetylphenyl)-1-methyl-1-azaspiro[4.5]deca-3,6,9-triene-2,8dione 3h, $95 \mathrm{mg}$, 94\% yield, yellow semi solid; $R_{f}=0.4$ (hexanes: EtOAc $\left.=1: 1\right) ;{ }^{1} \mathrm{H} \mathrm{NMR}(400 \mathrm{MHz}$, $\left.\mathrm{CDCl}_{3}\right) \delta 7.95-7.87(\mathrm{~m}, 2 \mathrm{H}), 7.28-7.27(\mathrm{~m}, 1 \mathrm{H}), 7.26-7.24(\mathrm{~m}, 1 \mathrm{H}), 6.56-6.47(\mathrm{~m}, 4 \mathrm{H}), 2.92(\mathrm{~s}$, $\mathrm{H}), 2.59(\mathrm{~s}, 3 \mathrm{H}), 2.52(\mathrm{~s}, 3 \mathrm{H}) ;{ }^{13} \mathrm{C} \mathrm{NMR}\left(101 \mathrm{MHz}, \mathrm{CDCl}_{3}\right) \delta 196.9,195.3,183.2,166.8,157.7$, 143.2, 138.0, 136.1, 134.8, 134.1, 128.3, 128.2, 67.4, 30.7, 26.6, 26.2; IR (KBr): vmax = 2924, 2854, 1698, 1381, 1249, 1047, $762 \mathrm{~cm}^{-1}$; HRMS (ESI): $\mathrm{m} / z$ calcd for $\mathrm{C}_{20} \mathrm{H}_{18} \mathrm{NO}_{4}(\mathrm{M}+\mathrm{H})^{+}: 336.1236$, found: 336.1236.

3-Acetyl-1-methyl-4-(3-(trifluoromethyl)phenyl)-1-azaspiro[4.5]deca-3,6,9-triene-2,8-dione (3i):

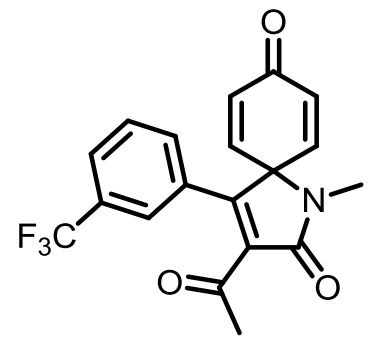

Following the general procedure, to the solution of N-(4-methoxyphenyl)-N-methyl-3-(3(trifluoromethyl)phenyl)propiolamide 1i (100 mg, $0.3 \mathrm{mmol}$ ), Pyruvic acid 2a ( $53 \mathrm{mg}, 0.6 \mathrm{mmol}$ ) and $\mathrm{K}_{2} \mathrm{~S}_{2} \mathrm{O}_{8}$ ( $399 \mathrm{mg}, 1.5 \mathrm{mmol}$ ) in $5 \mathrm{~mL}$ of Acetonitrile: water (9:1), silver carbonate (8.3 mg, $10 \mathrm{~mol} \%$ ) was added at room temperature. The reaction mixture was stirred at $70{ }^{\circ} \mathrm{C}$ for $8 \mathrm{~h}$. After completion of the reaction (monitored by TLC), the reaction mixture was concentrated under reduced pressure. The crude product was purified by column chromatography on silica gel (EtOAc-hexanes) to afford the corresponding product 3-acetyl-1-methyl-4-(3-(trifluoromethyl)phenyl)-1-azaspiro[4.5]deca-3,6,9triene-2,8-dione, 3i, $80 \mathrm{mg}, 91 \%$ yield, off-white solid; $R_{f}=0.4$ (hexanes: EtOAc = 9:1); mp 184-186 ${ }^{\circ} \mathrm{C} ;{ }^{1} \mathrm{H}$ NMR (400 MHz, $\left.\mathrm{CDCl}_{3}\right) \delta 7.66(\mathrm{~d}, J=7.8 \mathrm{~Hz}, 1 \mathrm{H}), 7.48(\mathrm{t}, J=7.8 \mathrm{~Hz}, 1 \mathrm{H}), 7.43(\mathrm{~s}, 1 \mathrm{H}), 7.37$ $(\mathrm{d}, J=7.8 \mathrm{~Hz}, 1 \mathrm{H}), 6.56-6.45(\mathrm{~m}, 4 \mathrm{H}), 2.93(\mathrm{~s}, 3 \mathrm{H}), 2.53(\mathrm{~s}, 3 \mathrm{H}) ;{ }^{13} \mathrm{C} \mathrm{NMR}\left(101 \mathrm{MHz}, \mathrm{CDCl}_{3}\right) \delta$ 195.1, 183.1, 166.8, 157.1, 143.1, 136.2, 134.2, 131.3, 131.1, 130.9, 130.8, 130.4, 129.0, 126.9, 124.8, 124.7, 67.5, 30.7, 26.2; ${ }^{19} \mathrm{~F}$ NMR (376 MHz, DMSO) $\delta-67.67$ (s, 3F); IR (KBr): vmax = 3018, 2924 , 2855, 1695, 1324, $753 \mathrm{~cm}^{-1}$; HRMS (ESI): $\mathrm{m} / z$ calcd for $\mathrm{C}_{19} \mathrm{H}_{15} \mathrm{NO}_{3} \mathrm{~F}_{3}(\mathrm{M}+\mathrm{H})^{+}: 362.1004$, found: 362.1013.

3-Acetyl-1-methyl-4-(naphthalen-1-yl)-1-azaspiro[4.5]deca-3,6,9-triene-2,8-dione (3j): 


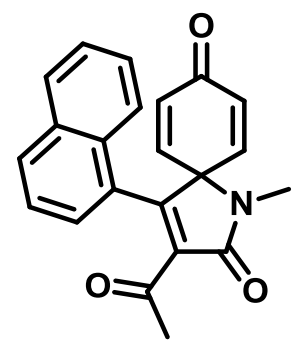

Following the general procedure, to the solution of $\mathrm{N}$-(4-methoxyphenyl)-N-methyl-3-(naphthalen-1yl)propiolamide $\mathbf{1 j}$ (95 mg, $0.3 \mathrm{mmol}$ ), Pyruvic acid $\mathbf{2 a}$ ( $53 \mathrm{mg}, 0.6 \mathrm{mmol}$ ) and $\mathrm{K}_{2} \mathrm{~S}_{2} \mathrm{O}_{8}$ ( $405 \mathrm{mg}, 1.5$ mmol) in $5 \mathrm{~mL}$ of Acetonitrile: water (9:1), silver carbonate (8.3 mg, $10 \mathrm{~mol} \%$ ) was added at room temperature. The reaction mixture was stirred at $70{ }^{\circ} \mathrm{C}$ for $8 \mathrm{~h}$. After completion of the reaction (monitored by TLC), the reaction mixture was concentrated under reduced pressure. The crude product was purified by column chromatography on silica gel (EtOAc-hexanes) to afford the corresponding product 3-acetyl-1-methyl-4-(naphthalen-1-yl)-1-azaspiro[4.5]deca-3,6,9-triene-2,8dione $3 \mathbf{j}, 88 \mathrm{mg}, 85 \%$ yield, off-white solid; $R_{f}=0.4$ (hexanes: EtOAc $=1: 1$ ); mp: $132-136{ }^{\circ} \mathrm{C} ;{ }^{1} \mathrm{H}$ NMR $\left(400 \mathrm{MHz}, \mathrm{CDCl}_{3}\right) \delta 7.79(\mathrm{~d}, J=8.3 \mathrm{~Hz}, 2 \mathrm{H}), 7.46-7.39(\mathrm{~m}, 3 \mathrm{H}), 7.37-7.30(\mathrm{~m}, J=8.3,7.2 \mathrm{~Hz}$, 1H), $7.08(\mathrm{dd}, J=7.1,1.0 \mathrm{~Hz}, 1 \mathrm{H}), 6.68-6.37$ (m, 3H), 6.02 (d, $J=9.7 \mathrm{~Hz}, 1 \mathrm{H}), 2.93(\mathrm{~s}, 3 \mathrm{H}), 2.25$ (s, 3H); ${ }^{13} \mathrm{C}$ NMR $\left(101 \mathrm{MHz}, \mathrm{CDCl}_{3}\right) \delta 194.3,183.3,143.4,134.4,133.4,130.3,129.1,127.2,126.4$, 125.8, 124.3, 123.9, 68.8, 30.2, 26.5; IR (KBr): $v \max =3689,3019,2930,2358,1701,1216,751 \mathrm{~cm}^{-}$ ${ }^{1}$; HRMS (ESI): $\mathrm{m} / z$ calcd for $\mathrm{C}_{22} \mathrm{H}_{18} \mathrm{NO}_{3}(\mathrm{M}+\mathrm{H})^{+}: 344.1287$, found: 344.1292 .

3-Acetyl-1-methyl-4-(thiophen-2-yl)-1-azaspiro[4.5]deca-3,6,9-triene-2,8-dione (3k):

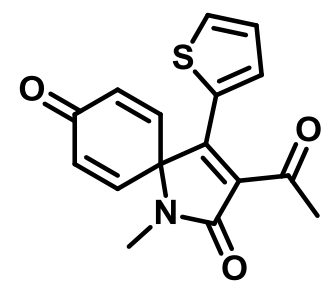

Following the general procedure, to the solution of $\mathrm{N}$-(4-methoxyphenyl)-N-methyl-3-(thiophen-2yl)propiolamide 1k (83 mg, $0.3 \mathrm{mmol}$ ), Pyruvic acid $2 \mathbf{a}$ ( $53 \mathrm{mg}, 0.6 \mathrm{mmol}$ ) and $\mathrm{K}_{2} \mathrm{~S}_{2} \mathrm{O}_{8}(411 \mathrm{mg}, 1.5$ mmol) in $5 \mathrm{~mL}$ of Acetonitrile: water (9:1), silver carbonate $(8.3 \mathrm{mg}, 10 \mathrm{~mol} \%)$ was added at room temperature. The reaction mixture was stirred at $70{ }^{\circ} \mathrm{C}$ for $8 \mathrm{~h}$. After completion of the reaction (monitored by TLC), the reaction mixture was concentrated under reduced pressure. The crude product was purified by column chromatography on silica gel (EtOAc-hexanes) to afford the corresponding product 3-acetyl-1-methyl-4-(thiophen-2-yl)-1-azaspiro[4.5]deca-3,6,9-triene-2,8dione 3k, $70 \mathrm{mg}, 78 \%$ yield, off-white solid; $R_{f}=0.4$ (hexanes: EtOAc $=9: 1$ ); mp: $184-186{ }^{\circ} \mathrm{C} ;{ }^{1} \mathrm{H}$ NMR (400 MHz, $\left.\mathrm{CDCl}_{3}\right) \delta 7.57(\mathrm{dd}, J=3.9,1.1 \mathrm{~Hz}, 1 \mathrm{H}), 7.50(\mathrm{dd}, J=5.1,1.1 \mathrm{~Hz}, 1 \mathrm{H}), 7.05(\mathrm{dd}, J=$ $5.1,3.9 \mathrm{~Hz}, 1 \mathrm{H}), 6.65-6.58(\mathrm{~m}, 2 \mathrm{H}), 6.58-6.51(\mathrm{~m}, 2 \mathrm{H}), 2.86(\mathrm{~s}, 3 \mathrm{H}), 2.61(\mathrm{~s}, 3 \mathrm{H}) ;{ }^{13} \mathrm{C}$ NMR $(101$ 
$\left.\mathrm{MHz}, \mathrm{CDCl}_{3}\right) \delta 197.6,183.8,167.3,148.5,144.7,133.7,132.1,131.4,130.9,127.9,66.4,30.9,25.5 ;$ IR (KBr): $v \max =3296,3111,3017,2924,1678,1380,758 \mathrm{~cm}^{-1}$; HRMS (ESI): $\mathrm{m} / \mathrm{z}$ calcd for $\mathrm{C}_{16} \mathrm{H}_{14} \mathrm{NO}_{3} \mathrm{~S}(\mathrm{M}+\mathrm{H})^{+}: 300.0694$, found: 300.0699 .

3-Acetyl-1,7-dimethyl-4-phenyl-1-azaspiro[4.5]deca-3,6,9-triene-2,8-dione (3I):

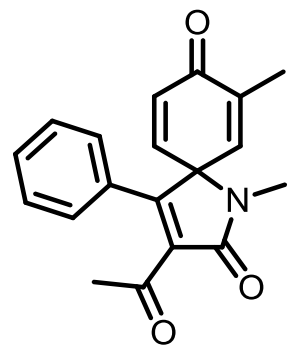

Following the general procedure, to the solution of N-(4-methoxy-3-methylphenyl)-N-methyl-3phenylpropiolamide 11 ( $85 \mathrm{mg}, 0.3 \mathrm{mmol}$ ), Pyruvic acid $\mathbf{2 a}$ ( $53 \mathrm{mg}, 0.6 \mathrm{mmol}$ ) and $\mathrm{K}_{2} \mathrm{~S}_{2} \mathrm{O}_{8}(409 \mathrm{mg}, 1.5$ $\mathrm{mmol})$ in $5 \mathrm{~mL}$ of Acetonitrile: water $(9: 1)$, silver carbonate $(8.3 \mathrm{mg}, 10 \mathrm{~mol} \%)$ was added at room temperature. The reaction mixture was stirred at $70{ }^{\circ} \mathrm{C}$ for $8 \mathrm{~h}$. After completion of the reaction (monitored by TLC), the reaction mixture was concentrated under reduced pressure. The crude product was purified by column chromatography on silica gel (EtOAc-hexanes) to afford the corresponding product 3-acetyl-1,7-dimethyl-4-phenyl-1-azaspiro[4.5]deca-3,6,9-triene-2,8-dione 31, $86 \mathrm{mg}, 83 \%$ yield, brown semi solid; $R_{f}=0.4$ (hexanes: EtOAc $\left.=9: 1\right) ;{ }^{1} \mathrm{H} \mathrm{NMR}\left(400 \mathrm{MHz}, \mathrm{CDCl}_{3}\right) \delta$ $7.41-7.35(\mathrm{~m}, 2 \mathrm{H}), 7.35-7.29(\mathrm{~m}, 2 \mathrm{H}), 7.17-7.11(\mathrm{~m}, 2 \mathrm{H}), 6.50-6.42(\mathrm{~m}, 1 \mathrm{H}), 6.32-6.26(\mathrm{~m}$, $1 \mathrm{H}), 2.88(\mathrm{~s}, 3 \mathrm{H}), 2.43(\mathrm{~s}, 3 \mathrm{H}), 1.93(\mathrm{~d}, J=1.5 \mathrm{~Hz}, 3 \mathrm{H}) ;{ }^{13} \mathrm{C} \mathrm{NMR}\left(101 \mathrm{MHz}, \mathrm{CDCl}_{3}\right) \delta 196.1,184.4$, 167.0, 158.6, 143.6, 141.1, 138.5, 135.5, 133.5, 130.3, 130.2, 128.5, 127.7, 67.9, 30.8, 26.0, 15.9; IR $(\mathrm{KBr}): v \max =3021,2358,1705,1382,1215,745 \mathrm{~cm}^{-1}$; HRMS (ESI): $\mathrm{m} / z$ calcd for $\mathrm{C}_{19} \mathrm{H}_{18} \mathrm{NO}_{3}$ $(\mathrm{M}+\mathrm{H})^{+}:$308.1287, found: 308.1277 .

\section{3-Acetyl-7-fluoro-1-methyl-4-phenyl-1-azaspiro[4.5]deca-3,6,9-triene-2,8-dione (3m):}

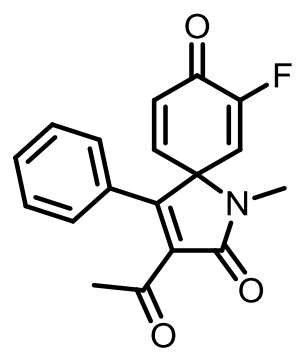

Following the general procedure, to the solution of N-(3-fluoro-4-methoxyphenyl)-N-methyl-3phenylpropiolamide $1 \mathrm{~m}$ (85mg, $0.3 \mathrm{mmol}$ ), Pyruvic acid 2a ( $53 \mathrm{mg}, 0.6 \mathrm{mmol}$ ) and $\mathrm{K}_{2} \mathrm{~S}_{2} \mathrm{O}_{8}(403 \mathrm{mg}$, $1.5 \mathrm{mmol})$ in $5 \mathrm{~mL}$ of Acetonitrile: water (9:1), silver carbonate $(8.3 \mathrm{mg}, 10 \mathrm{~mol} \%)$ was added at room temperature. The reaction mixture was stirred at $70{ }^{\circ} \mathrm{C}$ for $8 \mathrm{~h}$. After completion of the reaction (monitored by TLC), the reaction mixture was concentrated under reduced pressure. The crude product was purified by column chromatography on silica gel (EtOAc-hexanes) to afford the 
corresponding product 3-acetyl-7-fluoro-1-methyl-4-phenyl-1-azaspiro[4.5]deca-3,6,9-triene-2,8dione 3m, $80 \mathrm{mg}, 86 \%$ yield, brown semi solid; $R_{f}=0.4$ (hexanes: EtOAc $\left.=9: 1\right) ;{ }^{1} \mathrm{H} \mathrm{NMR}(400 \mathrm{MHz}$, $\left.\mathrm{CDCl}_{3}\right) \delta 7.45-7.40(\mathrm{~m}, 1 \mathrm{H}), 7.40-7.32(\mathrm{~m}, 2 \mathrm{H}), 7.19-7.13(\mathrm{~m}, 2 \mathrm{H}), 6.58-6.45(\mathrm{~m}, 2 \mathrm{H}), 6.11$ $(\mathrm{dd}, J=11.1,2.5 \mathrm{~Hz}, 1 \mathrm{H}), 2.92(\mathrm{~s}, 3 \mathrm{H}), 2.43(\mathrm{~s}, 3 \mathrm{H}) ;{ }^{13} \mathrm{C} \mathrm{NMR}\left(101 \mathrm{MHz}, \mathrm{CDCl}_{3}\right) \delta 195.6,176.65(\mathrm{~d}$, $\mathrm{J}=22.4 \mathrm{~Hz}$ ), 166.6, 157.6, 155.97 (d, J = 273.5 Hz), 145.1, 135.8, 132.8, 130.6, 129.8, 128.8, 127.7, $119.90(\mathrm{~d}, \mathrm{~J}=14.6 \mathrm{~Hz}), 68.85(\mathrm{~d}, \mathrm{~J}=8.2 \mathrm{~Hz}), 30.8,26.1 ;{ }^{19} \mathrm{~F}$ NMR $\left(376 \mathrm{MHz}, \mathrm{CDCl}_{3}\right) \delta-120.50(\mathrm{~s}$, 1F); IR (KBr): $v \max =3020,2928,1681,1631,1389,1217,740 \mathrm{~cm}^{-1}$; HRMS (ESI): $\mathrm{m} / z$ calcd for $\mathrm{C}_{18} \mathrm{H}_{14} \mathrm{NO}_{3} \mathrm{NaF}(\mathrm{M}+\mathrm{Na})^{+}: 334.0855$, found: 334.0859 .

\section{3-acetyl-1,6-dimethyl-4-phenyl-1-azaspiro[4.5]deca-3,6,9-triene-2,8-dione (3n):}

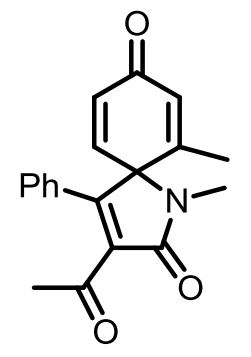

Following the general procedure, to the solution of $\mathrm{N}$-(4-methoxy-2-methylphenyl)-N-methyl-3phenylpropiolamide $\mathbf{1 p}$ ( $80 \mathrm{mg}, 0.3 \mathrm{mmol}$ ), pyruvic acid $\mathbf{2 b}$ ( $50 \mathrm{mg}, 0.6 \mathrm{mmol}$ ) and $\mathrm{K}_{2} \mathrm{~S}_{2} \mathrm{O}_{8}$ ( $385 \mathrm{mg}$, $1.5 \mathrm{mmol}$ ) in $5 \mathrm{~mL}$ of Acetonitrile: water (9:1), silver carbonate ( $8.3 \mathrm{mg}, 10 \mathrm{~mol} \%)$ was added at room temperature. The reaction mixture was stirred at $70{ }^{\circ} \mathrm{C}$ for $7 \mathrm{~h}$. After completion of the reaction (monitored by TLC), the reaction mixture was concentrated under reduced pressure. The crude product was purified by column chromatography on silica gel (EtOAc-hexanes) to afford the corresponding product 3-acetyl-1,6-dimethyl-4-phenyl-1-azaspiro[4.5]deca-3,6,9-triene-2,8-dione 3n, $72 \mathrm{mg}, 78 \%$ yield, yellow semisolid; $R_{f}=0.3$ (hexanes: EtOAc $\left.=5: 5\right) ;{ }^{1} \mathrm{H} \mathrm{NMR}\left(400 \mathrm{MHz}, \mathrm{CDCl}_{3}\right) \delta$ $7.43-7.38$ (m, 1H), $7.36-7.31(\mathrm{~m}, 2 \mathrm{H}), 7.17(\mathrm{dd}, J=5.3,3.3 \mathrm{~Hz}, 2 \mathrm{H}), 6.52-6.48(\mathrm{~m}, 2 \mathrm{H}), 6.39-$ $6.36(\mathrm{~m}, 1 \mathrm{H}), 2.81(\mathrm{~s}, 3 \mathrm{H}), 2.46(\mathrm{~s}, 3 \mathrm{H}), 1.79(\mathrm{~d}, J=1.4 \mathrm{~Hz}, 3 \mathrm{H}) ;{ }^{13} \mathrm{C} \mathrm{NMR}\left(101 \mathrm{MHz}, \mathrm{CDCl}_{3}\right) \delta$ 196.2, 184.3, 167.4, 157.8, 152.2, 144.0, 136.4, 133.2, 132.4, 130.6, 129.9, 128.8, 127.6, 69.5, 30.9, 25.6, 17.8; IR (KBr): vmax $=3746,2917,1730,1554,768 \mathrm{~cm}^{-1}$; HRMS (ESI): $\mathrm{m} / \mathrm{z}$ calcd for $\mathrm{C}_{19} \mathrm{H}_{17} \mathrm{NO}_{3}(\mathrm{M}+\mathrm{H})^{+}:$308.1287, found: 308.1286 .

3-Acetyl-1-benzyl-4-phenyl-1-azaspiro[4.5]deca-3,6,9-triene-2,8-dione (3o):<smiles>CC(=O)C1=C(c2ccccc2)C2(C=CC(=O)C=C2)N(Cc2ccccc2)C1=O</smiles> 
Following the general procedure, to the solution of N-benzyl-N-(4-methoxyphenyl)-3phenylpropiolamide 1q (105 mg, $0.3 \mathrm{mmol}$ ), Pyruvic acid 2a ( $53 \mathrm{mg}, 0.6 \mathrm{mmol}$ ) and $\mathrm{K}_{2} \mathrm{~S}_{2} \mathrm{O}_{8}(414 \mathrm{mg}$, $1.5 \mathrm{mmol}$ ) in $5 \mathrm{~mL}$ of Acetonitrile: water (9:1), silver carbonate (8.3 $\mathrm{mg}, 10 \mathrm{~mol} \%$ ) was added at room temperature. The reaction mixture was stirred at $70{ }^{\circ} \mathrm{C}$ for $8 \mathrm{~h}$. After completion of the reaction (monitored by TLC), the reaction mixture was concentrated under reduced pressure. The crude product was purified by column chromatography on silica gel (EtOAc-hexanes) to afford the corresponding product 3-acetyl-1-benzyl-4-phenyl-1-azaspiro[4.5]deca-3,6,9-triene-2,8-dione 3o, 94 $\mathrm{mg}, 85 \%$ yield, brown semi solid; $R_{f}=0.4$ (hexanes: EtOAc $\left.=9: 1\right) ;{ }^{1} \mathrm{H} \mathrm{NMR}\left(400 \mathrm{MHz}, \mathrm{CDCl}_{3}\right) \delta 7.33$ - $7.26(\mathrm{~m}, 1 \mathrm{H}), 7.23$ (d, J = 7.7 Hz, 2H), $7.21-7.15$ (m, 5H), $7.07-6.99(\mathrm{~m}, 2 \mathrm{H}), 6.32-6.24(\mathrm{~m}$, $2 \mathrm{H}), 6.18(\mathrm{~d}, J=10.2 \mathrm{~Hz}, 2 \mathrm{H}), 4.48(\mathrm{~s}, 2 \mathrm{H}), 2.36(\mathrm{~s}, 3 \mathrm{H}) ;{ }^{13} \mathrm{C} \mathrm{NMR}\left(101 \mathrm{MHz}, \mathrm{CDCl}_{3}\right) \delta 195.8$, 183.8, 167.2, 158.7, 144.0, 137.1, 135.5, 132.9, 130.3, 130.0, 128.9, 128.6, 128.5, 128.0, 127.8, 67.8, 44.8, 30.8; IR (KBr): vmax $=3020,2928,1681,1631,1389,1217,740 \mathrm{~cm}^{-1}$; HRMS (ESI): $\mathrm{m} / z$ calcd for $\mathrm{C}_{24} \mathrm{H}_{20} \mathrm{NO}_{3}(\mathrm{M}+\mathrm{H})^{+}: 370.1437$, found: 370.1443 .

\section{3-acetyl-4-phenyl-1-tosyl-1-azaspiro[4.5]deca-3,6,9-triene-2,8-dione (3p):}

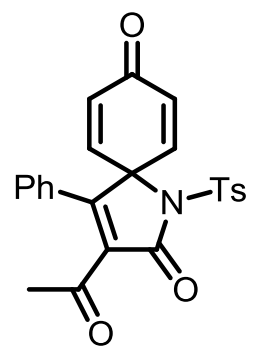

Following the general procedure, to the solution of $\mathrm{N}$-(4-methoxyphenyl)-3-phenyl-N-tosylpropiolamide 1r (122 mg, $0.3 \mathrm{mmol})$, pyruvic acid $2 \mathbf{b}(53 \mathrm{mg}, 0.6 \mathrm{mmol})$ and $\mathrm{K}_{2} \mathrm{~S}_{2} \mathrm{O}_{8}(405 \mathrm{mg}, 1.5 \mathrm{mmol})$ in $5 \mathrm{~mL}$ of Acetonitrile: water (9:1), silver carbonate $(8.3 \mathrm{mg}, 10 \mathrm{~mol} \%)$ was added at room temperature. The reaction mixture was stirred at $70{ }^{\circ} \mathrm{C}$ for $6 \mathrm{~h}$. After completion of the reaction (monitored by TLC), the reaction mixture was concentrated under reduced pressure. The crude product was purified by column chromatography on silica gel (EtOAc-hexanes) to afford the corresponding product 3-acetyl-4phenyl-1-tosyl-1-azaspiro[4.5]deca-3,6,9-triene-2,8-dione 3p, $110 \mathrm{mg}, 84 \%$ yield, white solid; $R_{f}=0.3$ (hexanes: EtOAc = 7:3); mp: $169-171{ }^{\circ} \mathrm{C}{ }^{1} \mathrm{H}$ NMR $\left(400 \mathrm{MHz}, \mathrm{CDCl}_{3}\right) \delta 8.03-7.99(\mathrm{~m}, 2 \mathrm{H}), 7.42-$ $7.37(\mathrm{~m}, 3 \mathrm{H}), 7.34-7.29(\mathrm{~m}, 2 \mathrm{H}), 7.04-7.01(\mathrm{~m}, 2 \mathrm{H}), 6.70-6.64(\mathrm{~m}, 2 \mathrm{H}), 6.48-6.43(\mathrm{~m}, 2 \mathrm{H})$, 2.48 (s, 3H), 2.30 (s, 3H); ${ }^{13} \mathrm{C}$ NMR (101 MHz, $\left.\mathrm{CDCl}_{3}\right) \delta 193.7,183.3,164.4,162.1,146.1,141.9$, 135.2, 134.0, 132.9, 130.6, 129.9, 128.7, 128.6, 128.4, 127.9, 69.0, 30.6, 21.8; IR (KBr): vmax = 3068, 2922, 1739, 1368, 1178, $758 \mathrm{~cm}^{-1}$; HRMS (ESI): $\mathrm{m} / z$ calcd for $\mathrm{C}_{24} \mathrm{H}_{19} \mathrm{NO}_{5} \mathrm{~S}(\mathrm{M}+\mathrm{H})^{+}: 434.1062$, found: 434.1053.

\section{3-Acetyl-1-(2-iodobenzyl)-4-phenyl-1-azaspiro[4.5]deca-3,6,9-triene-2,8-dione (3q):}




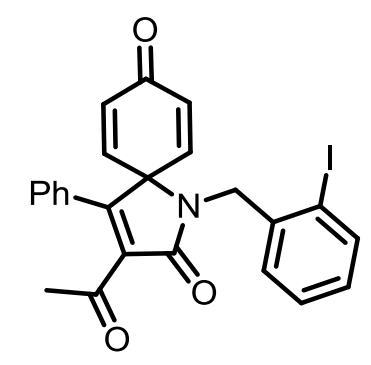

Following the general procedure, to the solution of N-(2-iodobenzyl)-N-(4-methoxyphenyl)-3phenylpropiolamide 1t (141 mg, $0.3 \mathrm{mmol}$ ), Pyruvic acid 2a ( $53 \mathrm{mg}, 0.6 \mathrm{mmol}$ ) and $\mathrm{K}_{2} \mathrm{~S}_{2} \mathrm{O}_{8}(516 \mathrm{mg}$, $1.5 \mathrm{mmol}$ ) in $5 \mathrm{~mL}$ of Acetonitrile: water (9:1), silver carbonate $(8.3 \mathrm{mg}, 10 \mathrm{~mol} \%)$ was added at room temperature. The reaction mixture was stirred at $70{ }^{\circ} \mathrm{C}$ for $8 \mathrm{~h}$. After completion of the reaction (monitored by TLC), the reaction mixture was concentrated under reduced pressure. The crude product was purified by column chromatography on silica gel (EtOAc-hexanes) to afford the corresponding product 3-Acetyl-1-(2-iodobenzyl)-4-phenyl-1-azaspiro[4.5]deca-3,6,9-triene-2,8dione 3q, $120 \mathrm{mg}, 80 \%$ yield, brown semi solid; $R_{f}=0.4$ (hexanes: EtOAc $\left.=9: 1\right) ;{ }^{1} \mathrm{H} \mathrm{NMR}(400 \mathrm{MHz}$, $\left.\mathrm{CDCl}_{3}\right) \delta 7.70(\mathrm{dd}, J=7.9,1.1 \mathrm{~Hz}, 1 \mathrm{H}), 7.34-7.27(\mathrm{~m}, 2 \mathrm{H}), 7.26-7.19(\mathrm{~m}, 3 \mathrm{H}), 7.04$ (dd, $J=5.3$, $3.3 \mathrm{~Hz}, 2 \mathrm{H}), 6.89(\mathrm{td}, J=7.7,1.6 \mathrm{~Hz}, 2 \mathrm{H}), 6.34-6.23(\mathrm{~m}, 1 \mathrm{H}), 6.23-6.13(\mathrm{~m}, 2 \mathrm{H}), 4.65(\mathrm{~s}, 2 \mathrm{H})$, $2.38(\mathrm{~s}, J=5.0 \mathrm{~Hz}, 3 \mathrm{H}) ;{ }^{13} \mathrm{C} \mathrm{NMR}\left(101 \mathrm{MHz}, \mathrm{CDCl}_{3}\right) \delta 195.7,183.8,167.2,159.1,143.1,139.6$, 139.4, 135.3, 133.3, 131.1, 130.3, 130.2, 130.1, 129.9, 129.7, 128.7, 128.5, 127.8, 99.6, 67.7, 49.1, 30.8; IR (KBr): vmax $=3369,3017,2925,2855,1705,1388,755 \mathrm{~cm}^{-1}$; HRMS (ESI): $\mathrm{m} / z$ calcd for $\mathrm{C}_{24} \mathrm{H}_{19} \mathrm{NO}_{3} \mathrm{I}(\mathrm{M}+\mathrm{H})^{+}:$496.0410, found: 496.0414 .

1-Methyl-4-phenyl-3-propionyl-1-azaspiro[4.5]deca-3,6,9-triene-2,8-dione (3r):

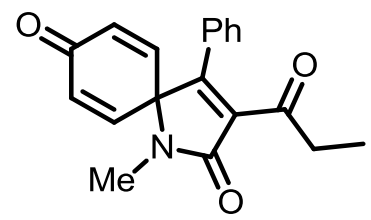

Following the general procedure, to the solution of N-(4-methoxyphenyl)-N-methyl-3phenylpropiolamide $\mathbf{1 a}$ ( $83 \mathrm{mg}, 0.3 \mathrm{mmol}$ ), 2-oxobutanoic acid $\mathbf{2 b}$ ( $62 \mathrm{mg}, 0.6 \mathrm{mmol}$ ) and $\mathrm{K}_{2} \mathrm{~S}_{2} \mathrm{O}_{8}(421$ $\mathrm{mg}, 1.5 \mathrm{mmol}$ ) in $5 \mathrm{~mL}$ of Acetonitrile: water (9:1), silver carbonate (8.3 mg, $10 \mathrm{~mol} \%)$ was added at room temperature. The reaction mixture was stirred at $70{ }^{\circ} \mathrm{C}$ for $8 \mathrm{~h}$. After completion of the reaction (monitored by TLC), the reaction mixture was concentrated under reduced pressure. The crude product was purified by column chromatography on silica gel (EtOAc-hexanes) to afford the corresponding product 1-methyl-4-phenyl-3-propionyl-1-azaspiro[4.5]deca-3,6,9-triene-2,8-dione 3r, 83 $\mathrm{mg}$, 90\% yield, brown semi solid; $R_{f}=0.4$ (hexanes: EtOAc $\left.=9: 1\right) ;{ }^{1} \mathrm{H} \mathrm{NMR}\left(400 \mathrm{MHz}, \mathrm{CDCl}_{3}\right) \delta 7.45$ $-7.38(\mathrm{~m}, 1 \mathrm{H}), 7.38-7.31(\mathrm{~m}, 2 \mathrm{H}), 7.23-7.17(\mathrm{~m}, 2 \mathrm{H}), 6.58-6.46(\mathrm{~m}, 4 \mathrm{H}), 2.91$ (s, 3H), 2.79 (q, $J$ $=7.2 \mathrm{~Hz}, 2 \mathrm{H}), 1.07(\mathrm{t}, J=7.2 \mathrm{~Hz}, 3 \mathrm{H}) ;{ }^{13} \mathrm{C} \mathrm{NMR}\left(101 \mathrm{MHz}, \mathrm{CDCl}_{3}\right) \delta 199.4,183.6,167.1,157.1$, 144.0, 136.0, 133.7, 130.3, 130.2, 128.6, 127.8, 67.4, 36.5, 26.0, 7.3; IR (KBr): vmax = 3008, 2923, 
2855, 1701, 1377, $757 \mathrm{~cm}^{-1}$; HRMS (ESI): $\mathrm{m} / z$ calcd for $\mathrm{C}_{19} \mathrm{H}_{18} \mathrm{NO}_{3}(\mathrm{M}+\mathrm{H})^{+}:$308.1287, found: 308.1281 .

\section{3-Heptanoyl-1-methyl-4-phenyl-1-azaspiro[4.5]deca-3,6,9-triene-2,8-dione (3s):}

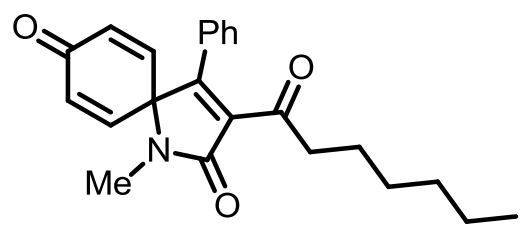

Following the general procedure, to the solution of $\mathrm{N}$-(4-methoxyphenyl)-N-methyl-3phenylpropiolamide 1a (83 mg, $0.3 \mathrm{mmol}$ ),2-oxooctanoic acid $2 \mathbf{c}$ ( $95 \mathrm{mg}, 0.6 \mathrm{mmol}$ ) and $\mathrm{K}_{2} \mathrm{~S}_{2} \mathrm{O}_{8}(421$ $\mathrm{mg}, 1.5 \mathrm{mmol}$ ) in $5 \mathrm{~mL}$ of Acetonitrile: water (9:1), silver carbonate (8.3 mg, $10 \mathrm{~mol} \%$ ) was added at room temperature. The reaction mixture was stirred at $70{ }^{\circ} \mathrm{C}$ for $8 \mathrm{~h}$. After completion of the reaction (monitored by TLC), the reaction mixture was concentrated under reduced pressure. The crude product was purified by column chromatography on silica gel (EtOAc-hexanes) to afford the corresponding product 3-heptanoyl-1-methyl-4-phenyl-1-azaspiro[4.5]deca-3,6,9-triene-2,8-dione 3s, $93 \mathrm{mg}, 85 \%$ yield, off white solid; $R_{f}=0.4$ (hexanes: EtOAc $=9: 1$ ); ${ }^{1} \mathrm{H}$ NMR $\left(400 \mathrm{MHz}, \mathrm{CDCl}_{3}\right) \delta 7.43$ - $7.36(\mathrm{~m}, J=6.4,3.7,1.3 \mathrm{~Hz}, 1 \mathrm{H}), 7.35-7.30(\mathrm{~m}, 2 \mathrm{H}), 7.18(\mathrm{dd}, J=8.3,1.2 \mathrm{~Hz}, 2 \mathrm{H}), 6.56-6.45$ (m, 4H), 2.89 (s, 3H), $2.74(\mathrm{t}, J=7.3 \mathrm{~Hz}, 2 \mathrm{H}), 1.64-1.48(\mathrm{~m}, 2 \mathrm{H}), 1.29-1.19(\mathrm{~m}, 6 \mathrm{H}), 0.84$ (t, $J=$ $6.9 \mathrm{~Hz}, 3 \mathrm{H}) ;{ }^{13} \mathrm{C} \mathrm{NMR}\left(101 \mathrm{MHz}, \mathrm{CDCl}_{3}\right) \delta 199.19,183.63,167.11,156.92,144.03,136.3,133.7$, 130.3, 130.2, 128.6, 127.8, 67.4, 43.2, 31.5, 28.6, 26.0, 23.2, 22.5, 14.0; IR (KBr): $v \max =3016$, 2924, 2840, 1670, 1211, 877, $743 \mathrm{~cm}^{-1}$; HRMS (ESI): $\mathrm{m} / z$ calcd for $\mathrm{C}_{23} \mathrm{H}_{26} \mathrm{NO}_{3}(\mathrm{M}+\mathrm{H})^{+}: 364.1913$, found: 364.1914 .

1-Methyl-3-(3-methylbutanoyl)-4-phenyl-1-azaspiro[4.5]deca-3,6,9-triene-2,8-dione (3t):

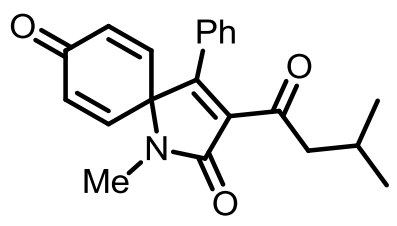

Following the general procedure, to the solution of N-(4-methoxyphenyl)-N-methyl-3phenylpropiolamide 1a $(83 \mathrm{mg}, 0.3 \mathrm{mmol}$ ), 4-methyl-2-oxovaleric acid $2 \mathbf{d}$ ( $71 \mathrm{mg}, 0.6 \mathrm{mmol}$ ) and $\mathrm{K}_{2} \mathrm{~S}_{2} \mathrm{O}_{8}$ ( $421 \mathrm{mg}, 1.5 \mathrm{mmol}$ ) in $5 \mathrm{~mL}$ of Acetonitrile: water (9:1), silver carbonate (8.3 mg, $10 \mathrm{~mol} \%$ ) was added at room temperature. The reaction mixture was stirred at $70{ }^{\circ} \mathrm{C}$ for $8 \mathrm{~h}$. After completion of the reaction (monitored by TLC), the reaction mixture was concentrated under reduced pressure. The crude product was purified by column chromatography on silica gel (EtOAc-hexanes) to afford the corresponding product 1-methyl-3-(3-methylbutanoyl)-4-phenyl-1-azaspiro[4.5]deca-3,6,9-triene-2,8dione 3t, $87 \mathrm{mg}, 86 \%$ yield, brown semi solid; $R_{f}=0.4$ (hexanes: EtOAc $\left.=9: 1\right) ;{ }^{1} \mathrm{H}$ NMR $(500 \mathrm{MHz}$, $\left.\mathrm{CDCl}_{3}\right) \delta 7.38(\mathrm{t}, J=7.4 \mathrm{~Hz}, 1 \mathrm{H}), 7.32(\mathrm{t}, J=7.6 \mathrm{~Hz}, 2 \mathrm{H}), 7.20-7.15(\mathrm{~m}, 2 \mathrm{H}), 6.56-6.45(\mathrm{~m}, J=$ 
10.4, $2.1 \mathrm{~Hz}, 4 \mathrm{H}), 2.89(\mathrm{~s}, 3 \mathrm{H}), 2.60(\mathrm{~d}, J=6.8 \mathrm{~Hz}, 2 \mathrm{H}), 2.18-2.07(\mathrm{~m}, 1 \mathrm{H}), 0.86(\mathrm{~d}, J=6.7 \mathrm{~Hz}$, $6 \mathrm{H}) ;{ }^{13} \mathrm{C}$ NMR $\left(101 \mathrm{MHz}, \mathrm{CDCl}_{3}\right) \delta 198.8,183.6,167.1,156.8,144.0,136.5,133.7,130.3,130.1$, 128.5, 127.8, 67.4, 51.9, 26.0, 24.2, 22.5; IR (KBr): vmax =3019, 2926, 2860, 1680, 1217, 874, 743 $\mathrm{cm}^{-1}$; HRMS (ESI): $\mathrm{m} / z$ calcd for $\mathrm{C}_{21} \mathrm{H}_{22} \mathrm{NO}_{3}(\mathrm{M}+\mathrm{H})^{+}: 336.1600$, found: 336.1605 .

\section{3-Benzoyl-1-methyl-4-phenyl-1-azaspiro[4.5]deca-3,6,9-triene-2,8-dione (3u):}

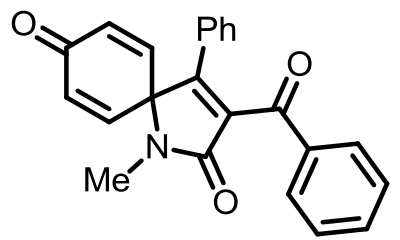

Following the general procedure, to the solution of $\mathrm{N}$-(4-methoxyphenyl)-N-methyl-3phenylpropiolamide 1a ( $83 \mathrm{mg}, 0.3 \mathrm{mmol}$ ), 2-oxo-2-phenylacetic acid $2 \mathbf{e}$ ( $92 \mathrm{mg}, 0.6 \mathrm{mmol}$ ) and $\mathrm{K}_{2} \mathrm{~S}_{2} \mathrm{O}_{8}$ ( $421 \mathrm{mg}, 1.5 \mathrm{mmol})$ in $5 \mathrm{~mL}$ of Acetonitrile: water (9:1), silver carbonate ( $8.3 \mathrm{mg}, 10 \mathrm{~mol} \%$ ) was added at room temperature. The reaction mixture was stirred at $70{ }^{\circ} \mathrm{C}$ for $8 \mathrm{~h}$. After completion of the reaction (monitored by TLC), the reaction mixture was concentrated under reduced pressure. The crude product was purified by column chromatography on silica gel (EtOAc-hexanes) to afford the corresponding product 3-benzoyl-1-methyl-4-phenyl-1-azaspiro[4.5]deca-3,6,9-triene-2,8-dione 3u, $75 \mathrm{mg}, 76 \%$ yield, brown semi solid; $R_{f}=0.4$ (hexanes: EtOAc $\left.=9: 1\right) ;{ }^{1} \mathrm{H} \mathrm{NMR}\left(500 \mathrm{MHz}, \mathrm{CDCl}_{3}\right) \delta$ $7.84(\mathrm{dd}, J=8.3,1.2 \mathrm{~Hz}, 2 \mathrm{H}), 7.57-7.52(\mathrm{~m}, 1 \mathrm{H}), 7.39$ (dd, $J=10.7,4.9 \mathrm{~Hz}, 3 \mathrm{H}), 7.30-7.27(\mathrm{~m}$, $2 \mathrm{H}), 7.22-7.18(\mathrm{~m}, 2 \mathrm{H}), 6.73-6.67(\mathrm{~m}, 2 \mathrm{H}), 6.62-6.56(\mathrm{~m}, 2 \mathrm{H}), 2.93(\mathrm{~s}, 3 \mathrm{H}) ;{ }^{13} \mathrm{C}$ NMR $(101$ $\left.\mathrm{MHz}, \mathrm{CDCl}_{3}\right) \delta 191.3,183.8,167.2,154.5,144.7,136.1,135.6,134.3,133.5,130.6,130.1,129.5$, 128.8, 128.7, 127.8, 67.3, 25.9; IR (KBr): vmax = 3016, 2924, 2856, 1697, 1655, 1376, $757 \mathrm{~cm}^{-1}$; HRMS (ESI): $m / z$ calcd for $\mathrm{C}_{23} \mathrm{H}_{18} \mathrm{NO}_{3}(\mathrm{M}+\mathrm{H})^{+}: 356.1293$, found: 356.1287 .

\section{General procedure for synthesis of 3-alkyl $[4,5]$ spirotrienones:}

To a stirred solution of N-(4-methoxyaryl)-propiolamide 1 (0.3 mmol), Carboxylic acid 4 (0.6 mmol) and $\mathrm{K}_{2} \mathrm{~S}_{2} \mathrm{O}_{8}(1.5 \mathrm{mmol})$, in $5 \mathrm{~mL}$ of Acetonitrile: water (1:1) in a reaction vial. Then, silver carbonate $(10 \mathrm{~mol} \%)$ was added at room temperature. The reaction mixture stirred at $70{ }^{\circ} \mathrm{C}$ (oil bath temperature) for 6-9 h. After completion of the reaction (monitored by TLC) the mixture was concentrated under reduced pressure. The crude product was purified by column chromatography on silica gel by using EtOAc: hexanes as an eluent to afford the corresponding 3-acyl[4,5] spirotrienones.

\section{1,3-Dimethyl-4-phenyl-1-azaspiro[4.5]deca-3,6,9-triene-2,8-dione (5a):}




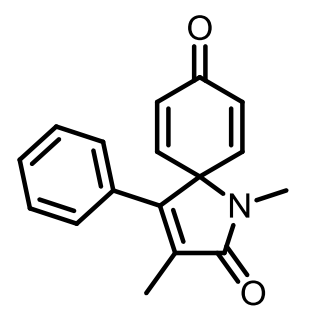

Following the general procedure, to the solution of $\mathrm{N}$-(4-methoxyphenyl)-N-methyl-3phenylpropiolamide 1a ( $83 \mathrm{mg}, 0.3 \mathrm{mmol}$ ), Acetic acid $4 \mathbf{a}$ ( $37 \mathrm{mg}, 0.6 \mathrm{mmol}$ ) and $\mathrm{K}_{2} \mathrm{~S}_{2} \mathrm{O}_{8}(421 \mathrm{mg}, 1.5$ $\mathrm{mmol})$ in $5 \mathrm{~mL}$ of Acetonitrile: water (1:1), silver carbonate $(8.3 \mathrm{mg}, 10 \mathrm{~mol} \%)$ was added at room temperature. The reaction mixture was stirred at $70{ }^{\circ} \mathrm{C}$ for $7 \mathrm{~h}$. After completion of the reaction (monitored by TLC), the reaction mixture was concentrated under reduced pressure. The crude product was purified by column chromatography on silica gel (EtOAc-hexanes) to afford the corresponding product 1,3-dimethyl-4-phenyl-1-azaspiro[4.5]deca-3,6,9-triene-2,8-dione 5a, $59 \mathrm{mg}$, $74 \%$ yield, Brown semisolid, $\mathrm{R}_{f}=0.5$ (Petroleum ether : EtOAc $\left.=7: 3\right) ;{ }^{1} \mathrm{H}$ NMR $\delta(300 \mathrm{MHz}$, CDCl3) $\delta 7.27(\mathrm{~d}, \mathrm{~J}=3.2 \mathrm{~Hz}, 3 \mathrm{H}), 7.12(\mathrm{~d}, \mathrm{~J}=3.6 \mathrm{~Hz}, 2 \mathrm{H}), 6.40(\mathrm{q}, \mathrm{J}=10.2 \mathrm{~Hz}, 4 \mathrm{H}), 2.82$ (s, 3H), $1.92(\mathrm{~s}, 3 \mathrm{H}) ;{ }^{13} \mathrm{C} \mathrm{NMR} \delta\left(101 \mathrm{MHz}, \mathrm{CDCl}_{3}\right) \delta 184.1,170.7,150.6,145.6,138.3,137.2,133.1,131.0$, 129.3, 128.6, 128.5, 128.3, 126.4, 67.7, 30.9, 26.1; IR (KBr): vmax =3409, 3067, 1685, 1389, 1063 , $761 \mathrm{~cm}^{-1}$; MS (ESI): m/z 266(M+H) ${ }^{+}$; HRMS (ESI) : m/z calcd for $\mathrm{C}_{17} \mathrm{H}_{16} \mathrm{NO}_{2}(\mathrm{M}+\mathrm{H})+: 266.1181$, Found :266.1174.

3-Ethyl-1-methyl-4-phenyl-1-azaspiro[4.5]deca-3,6,9-triene-2,8-dione (5b):

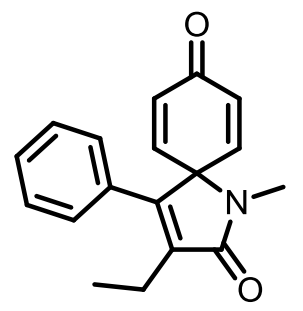

Following the general procedure, to the solution of N-(4-methoxyphenyl)-N-methyl-3phenylpropiolamide 1a ( $83 \mathrm{mg}, 0.3 \mathrm{mmol}$ ), Propionic acid $4 \mathbf{b}$ ( $55 \mathrm{mg}, 0.6 \mathrm{mmol}$ ) and $\mathrm{K}_{2} \mathrm{~S}_{2} \mathrm{O}_{8}(421 \mathrm{mg}$, $1.5 \mathrm{mmol}$ ) in $5 \mathrm{~mL}$ of Acetonitrile: water (1:1), silver carbonate (8.3 $\mathrm{mg}, 10 \mathrm{~mol} \%$ ) was added at room temperature. The reaction mixture was stirred at $70{ }^{\circ} \mathrm{C}$ for $7 \mathrm{~h}$. After completion of the reaction (monitored by TLC), the reaction mixture was concentrated under reduced pressure. The crude product was purified by column chromatography on silica gel (EtOAc-hexanes) to afford the corresponding product 3-ethyl-1-methyl-4-phenyl-1-azaspiro[4.5]deca-3,6,9-triene-2,8-dione 5b, 65 $\mathrm{mg}, 78 \%$ yield, off white solid, mp: $136-138{ }^{\circ} \mathrm{C}, \mathrm{R}_{f}=0.3$ (Petroleum ether : EtOAc $=7: 3$ ); ${ }^{1} \mathrm{H} \mathrm{NMR}$ (400 MHz, CDCl3) $\delta 7.38-7.30(\mathrm{~m}, 3 \mathrm{H}), 7.16-7.12(\mathrm{~m}, 2 \mathrm{H}), 6.52-6.47(\mathrm{~m}, 2 \mathrm{H}), 6.45-6.39(\mathrm{~m}$, 2H), $2.90(\mathrm{~s}, 3 \mathrm{H}), 2.37$ (q, J = 7.5 Hz, 2H), $1.12(\mathrm{t}, \mathrm{J}=9.6,5.5 \mathrm{~Hz}, 3 \mathrm{H}) ;{ }^{13} \mathrm{C} \mathrm{NMR}\left(101 \mathrm{MHz}, \mathrm{CDCl}_{3}\right)$ $\delta 184.2,170.9,148.9,145.9,139.8,132.0,129.1,128.5,128.2,67.4,25.9,18.6,13.2$; IR (KBr): vmax 
$=3479,2929,1685,1389,1064,877,710 \mathrm{~cm}^{-1}$; HRMS $($ ESI $): \mathrm{m} / \mathrm{z}$ calcd forC ${ }_{18} \mathrm{H}_{18} \mathrm{NO}_{2}(\mathrm{M}+\mathrm{H})^{+}$: 280.1338, Found: 280.1331.

\section{3-Isopropyl-1-methyl-4-phenyl-1-azaspiro[4.5]deca-3,6,9-triene-2,8-dione (5c):}

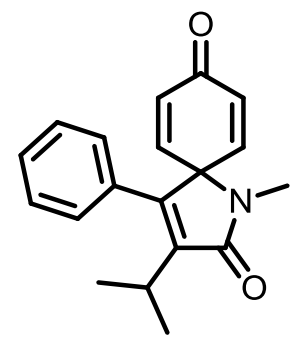

Following the general procedure, to the solution of $\mathrm{N}$-(4-methoxyphenyl)-N-methyl-3phenylpropiolamide1a (83 mg, $0.3 \mathrm{mmol}$ ), Isobutyric acid $4 \mathbf{c}$ ( $55 \mathrm{mg}, 0.6 \mathrm{mmol}$ ) and $\mathrm{K}_{2} \mathrm{~S}_{2} \mathrm{O}_{8}(421 \mathrm{mg}$, $1.5 \mathrm{mmol})$ in $5 \mathrm{~mL}$ of Acetonitrile: water (1:1), silver carbonate $(8.3 \mathrm{mg}, 10 \mathrm{~mol} \%)$ was added at room temperature. The reaction mixture was stirred at $70{ }^{\circ} \mathrm{C}$ for $8 \mathrm{~h}$. After completion of the reaction (monitored by TLC), the reaction mixture was concentrated under reduced pressure. The crude product was purified by column chromatography on silica gel (EtOAc-hexanes) to afford the corresponding product 3-isopropyl-1-methyl-4-phenyl-1-azaspiro[4.5]deca-3,6,9-triene-2,8-dione 5c, $73 \mathrm{mg}, 83 \%$ yield, mp: $106-108{ }^{\circ} \mathrm{C}, \mathrm{R}_{f}=0.4$ (Petroleum ether : EtOAc $=7: 3$ ); ${ }^{1} \mathrm{H}$ NMR $(400 \mathrm{MHz}$, $\left.\mathrm{CDCl}_{3}\right) \delta 7.37-7.28(\mathrm{~m}, 3 \mathrm{H}), 7.10-7.04(\mathrm{~m}, 2 \mathrm{H}), 6.52-6.45(\mathrm{~m}, 2 \mathrm{H}), 6.42-6.35(\mathrm{~m}, 2 \mathrm{H}), 2.88(\mathrm{~s}$, $3 \mathrm{H}), 2.85-2.75(\mathrm{~m}, 1 \mathrm{H}), 1.20(\mathrm{~d}, J=7.0 \mathrm{~Hz}, 6 \mathrm{H}) ;{ }^{13} \mathrm{C} \mathrm{NMR} \delta\left(125 \mathrm{MHz}, \mathrm{CDCl}_{3}\right) \delta 184.2,170.3$, 148.3, 145.9, 142.9, 132.0, 131.6, 128.9, 128.5, 128.3, 67.4, 26.7, 25.9, 20.7; IR (KBr): vmax = 3510, 2966, 1680, 1382, 1051, $875 \mathrm{~cm}^{-1}$ HRMS (ESI) : m/z calcd for $\mathrm{C}_{19} \mathrm{H}_{20} \mathrm{NO}_{2}(\mathrm{M}+\mathrm{H})^{+}: 294.1494$, Found : 294.1505.

\section{3-(tert-butyl)-1-methyl-4-phenyl-1-azaspiro[4.5]deca-3,6,9-triene-2,8-dione (5d):}

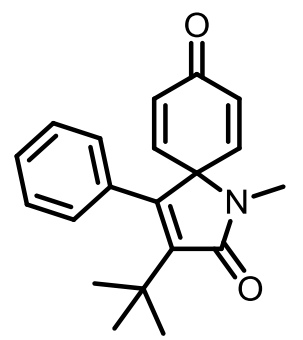

Following the general procedure, to the solution of N-(4-methoxyphenyl)-N-methyl-3phenylpropiolamide 1a ( $83 \mathrm{mg}, 0.3 \mathrm{mmol}$ ), Pivalic acid $4 \mathbf{d}$ ( $63 \mathrm{mg}, 0.6 \mathrm{mmol}$ ) and $\mathrm{K}_{2} \mathrm{~S}_{2} \mathrm{O}_{8}(421 \mathrm{mg}, 1.5$ $\mathrm{mmol})$ in $5 \mathrm{~mL}$ of Acetonitrile: water (1:1), silver carbonate $(8.3 \mathrm{mg}, 10 \mathrm{~mol} \%)$ was added at room temperature. The reaction mixture was stirred at $70{ }^{\circ} \mathrm{C}$ for $7 \mathrm{~h}$. After completion of the reaction (monitored by TLC), the reaction mixture was concentrated under reduced pressure. The crude product was purified by column chromatography on silica gel (EtOAc-hexanes) to afford the corresponding product 3-(tert-butyl)-1-methyl-4-phenyl-1-azaspiro[4.5]deca-3,6,9-triene-2,8-dione 
5d, $80 \mathrm{mg}, 87 \%$ yield, white solid; $\mathrm{mp} 178-180{ }^{\circ} \mathrm{C}, \mathrm{R}_{f}=0.3$ ( Petroleum ether : EtOAc $=7: 3$ ); ${ }^{1} \mathrm{H}$ NMR- (500 MHz, CDCl3) $\delta 7.27$ - $7.09(\mathrm{~m}, 3 \mathrm{H}), 6.92-6.88(\mathrm{~m}, 2 \mathrm{H}), 6.41-6.38(\mathrm{~m}, 2 \mathrm{H}), 6.26-$ $6.22(\mathrm{~m}, 2 \mathrm{H}), 2.81(\mathrm{~s}, 3 \mathrm{H}), 1.08(\mathrm{~s}, 9 \mathrm{H}) ;{ }^{13} \mathrm{C} \mathrm{NMR}(101 \mathrm{MHz}, \mathrm{CDCl} 3) \delta 184.1,170.4,147.6,145.9$, 144.2, 133.0, 132.9, 129.3, 128.4, 127.3, 67.7, 34.5, 29.6, 26.2; IR (KBr): vmax = 3354, 2962, 1680, 1380, 1051, $739 \mathrm{~cm}^{-1}$; HRMS (ESI) : m/z calcd for $\mathrm{C}_{20} \mathrm{H}_{22} \mathrm{NO}_{2}(\mathrm{M}+\mathrm{H})^{+}: 308.1651$, Found: 308.1660 .

\section{3-Benzyl-1-methyl-4-phenyl-1-azaspiro[4.5]deca-3,6,9-triene-2,8-dione (5e):}

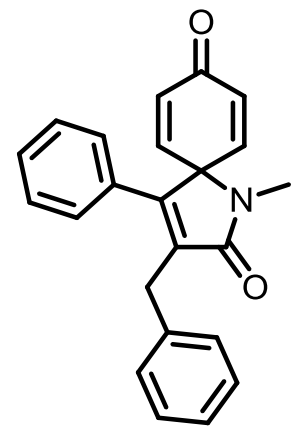

Following the general procedure, to the solution of $\mathrm{N}$-(4-methoxyphenyl)-N-methyl-3phenylpropiolamide 1a ( $83 \mathrm{mg}, 0.3 \mathrm{mmol}$ ), 2 Phenylacetic acid $4 \mathbf{e}\left(85 \mathrm{mg}, 0.6 \mathrm{mmol}\right.$ ) and $\mathrm{K}_{2} \mathrm{~S}_{2} \mathrm{O}_{8}(421$ $\mathrm{mg}, 1.5 \mathrm{mmol}$ ) in $5 \mathrm{~mL}$ of Acetonitrile: water (1:1), silver carbonate $(8.3 \mathrm{mg}, 10 \mathrm{~mol} \%)$ was added at room temperature. The reaction mixture was stirred at $70{ }^{\circ} \mathrm{C}$ for $7 \mathrm{~h}$. After completion of the reaction (monitored by TLC), the reaction mixture was concentrated under reduced pressure. The crude product was purified by column chromatography on silica gel (EtOAc-hexanes) to afford the corresponding product 3-benzyl-1-methyl-4-phenyl-1-azaspiro[4.5]deca-3,6,9-triene-2,8-dione 5e, 74 $\mathrm{mg}, 72 \%$ yield, yellow solid , mp:130 $-132{ }^{\circ} \mathrm{C} ; \mathrm{R}_{f}=0.4$ (Petroleum ether : EtOAc $=7: 3$ ); ${ }^{1} \mathrm{H}$ NMR $\left(400 \mathrm{MHz}, \mathrm{CDCl}_{3}\right) \delta 7.38-7.29(\mathrm{~m}, 3 \mathrm{H}), 7.22(\mathrm{~d}, J=7.4 \mathrm{~Hz}, 2 \mathrm{H}), 7.18(\mathrm{~d}, J=7.0 \mathrm{~Hz}, 1 \mathrm{H}), 7.14(\mathrm{~d}, J$ $=7.0 \mathrm{~Hz}, 2 \mathrm{H}), 7.12-7.07(\mathrm{~m}, 2 \mathrm{H}), 6.50(\mathrm{~d}, J=10.1 \mathrm{~Hz}, 2 \mathrm{H}), 6.41(\mathrm{~d}, J=10.1 \mathrm{~Hz}, 2 \mathrm{H}), 3.68(\mathrm{~s}, 2 \mathrm{H})$, 2.90 (s, H); ${ }^{13} \mathrm{C}$ NMR (101 MHz, CDCl3) $\delta 184.1,170.7,150.6,145.6,138.3,137.2,133.1,131.0$, 129.3, 128.6, 128.5, 128.3, 126.4, 67.7, 30.9, 26.1; IR (KBr): vmax $=3511,2931,1672,1054,700 \mathrm{~cm}^{-}$ ${ }^{1}$; ; HRMS (ESI) : m/z calcd forC ${ }_{23} \mathrm{H}_{20} \mathrm{NO}_{2}(\mathrm{M}+\mathrm{H})^{+}: 342.1494$, Found : 342.1505 .

3-Cyclohexyl-1-methyl-4-phenyl-1-azaspiro[4.5]deca-3,6,9-triene-2,8-dione (5f):

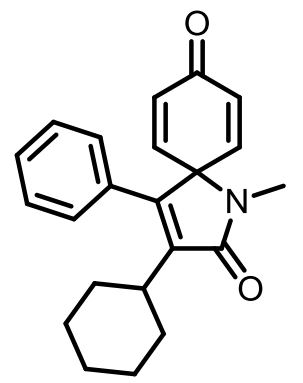


Following the general procedure, to the solution of $\mathrm{N}$-(4-methoxyphenyl)-N-methyl-3phenylpropiolamide 1a ( $83 \mathrm{mg}, 0.3 \mathrm{mmol})$, Cyclohexanecarboxylic acid $\mathbf{1 f}(80 \mathrm{mg}, 0.6 \mathrm{mmol})$ and $\mathrm{K}_{2} \mathrm{~S}_{2} \mathrm{O}_{8}$ ( $421 \mathrm{mg}, 1.5 \mathrm{mmol}$ ) in $5 \mathrm{~mL}$ of Acetonitrile: water (1:1), silver carbonate (8.3 mg, $10 \mathrm{~mol} \%$ ) was added at room temperature. The reaction mixture was stirred at $70{ }^{\circ} \mathrm{C}$ for $9 \mathrm{~h}$. After completion of the reaction (monitored by TLC), the reaction mixture was concentrated under reduced pressure. The crude product was purified by column chromatography on silica gel (EtOAc-hexanes) to afford the corresponding product 3-cyclohexyl-1-methyl-4-phenyl-1-azaspiro[4.5]deca-3,6,9-triene-2,8-dione 5f, $78 \mathrm{mg}, 78 \%$ yield, white solid, mp:130-132 ${ }^{\circ} \mathrm{C} ; \mathrm{R}_{f}=0.3$ (Petroleum ether : EtOAc $=5: 5$ ); ${ }^{1} \mathrm{H}$ NMR$\left(400 \mathrm{MHz}, \mathrm{CDCl}_{3}\right) \delta 7.37-7.29(\mathrm{~m}, 3 \mathrm{H}), 7.05(\mathrm{dd}, J=7.7,1.7 \mathrm{~Hz}, 2 \mathrm{H}), 6.47(\mathrm{dd}, J=10.5,2.2 \mathrm{~Hz}$, 2H), 6.37 (dd, $J=10.5,2.2 \mathrm{~Hz}, 2 \mathrm{H}), 2.87(\mathrm{~s}, 3 \mathrm{H}), 2.56-2.33(\mathrm{~m}, 1 \mathrm{H}), 1.83-1.64(\mathrm{~m}, 4 \mathrm{H}), 1.67-$ $1.48(\mathrm{~m}, 3 \mathrm{H}), 1.29-1.06$ (m, 3H); ${ }^{13} \mathrm{C}$ NMR- (101 MHz, CDCl3) $\delta 184.2,170.5,148.6,146.0,142.2$, 132.9, 131.6, 128.9, 128.6, 128.2, 67.4, 37.0, 30.4, 26.3, 25.9, 25.6; IR (KBr): vmax =3527, 3014, $2928,1678,1382,1064,751 \mathrm{~cm}^{-1}$; HRMS (ESI) : m/z calcd forC ${ }_{22} \mathrm{H}_{24} \mathrm{NO}_{2}(\mathrm{M}+\mathrm{H})^{+}: 334.1807$, Found : 334.1817.

\section{3-(Adamantan-1-yl)-1-methyl-4-phenyl-1-azaspiro[4.5]deca-3,6,9-triene-2,8-dione (5g):}

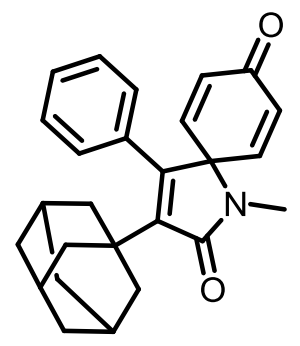

Following the general procedure, to the solution of $\mathrm{N}$-(4-methoxyphenyl)-N-methyl-3phenylpropiolamide 1a ( $83 \mathrm{mg}, 0.3 \mathrm{mmol}$ ), Adamantane 1- carboxylic acid $\mathbf{4 g}$ ( $112 \mathrm{mg}, 0.6 \mathrm{mmol}$ ) and $\mathrm{K}_{2} \mathrm{~S}_{2} \mathrm{O}_{8}(421 \mathrm{mg}, 1.5 \mathrm{mmol})$ in $5 \mathrm{~mL}$ of Acetonitrile: water (1:1), silver carbonate (8.3 $\left.\mathrm{mg}, 10 \mathrm{~mol} \%\right)$ was added at room temperature. The reaction mixture was stirred at $70{ }^{\circ} \mathrm{C}$ for $7 \mathrm{~h}$. After completion of the reaction (monitored by TLC), the reaction mixture was concentrated under reduced pressure. The crude product was purified by column chromatography on silica gel (EtOAc-hexanes) to afford the corresponding product 3-(adamantan-1-yl)-1-methyl-4-phenyl-1-azaspiro[4.5]deca-3,6,9-triene-2,8dione 5g, 95 mg, 82\% yield,off- white solid, mp: 80-82 ${ }^{\circ} \mathrm{C}$; $\mathrm{Rf}=0.3$ (Petroleum ether : EtOAc = 5:5 ); ${ }^{1} \mathrm{H}$ NMR (300 MHz, CDCl3) $\delta 7.31-7.19(\mathrm{~m}, 3 \mathrm{H}), 6.96(\mathrm{~d}, \mathrm{~J}=6.5 \mathrm{~Hz}, 2 \mathrm{H}), 6.46(\mathrm{~d}, \mathrm{~J}=10.0 \mathrm{~Hz}$, $2 \mathrm{H}), 6.29(\mathrm{~d}, \mathrm{~J}=10.0 \mathrm{~Hz}, 2 \mathrm{H}), 2.84(\mathrm{~s}, 3 \mathrm{H}), 1.86(\mathrm{~s}, 9 \mathrm{H}), 1.63(\mathrm{~s}, 6 \mathrm{H}) ;{ }^{13} \mathrm{C}$ NMR $(101 \mathrm{MHz}, \mathrm{CDCl} 3)$ $\delta 184.2,170.3,147.9,146.1,143.6,133.0,129.5,128.3,127.1,67.7,40.5,37.4,36.6,28.3$, 26.2; IR $(\mathrm{KBr}): \mathrm{vmax}=3476,2908,1681,1381,760 \mathrm{~cm}^{-1}$; HRMS (ESI) : m/z calcd for $\mathrm{C}_{26} \mathrm{H}_{28} \mathrm{NO}_{2}(\mathrm{M}+\mathrm{H})^{+}$: 386.2120, Found : 386.2132 .

\section{3-(3-Hydroxyadamantan-1-yl)-1-methyl-4-phenyl-1-azaspiro[4.5]deca-3,6,9-triene-2,8-dione}




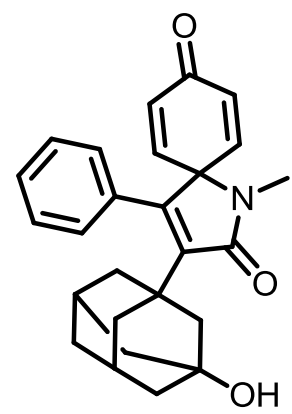

Following the general procedure, to the solution of $\mathrm{N}$-(4-methoxyphenyl)-N-methyl-3phenylpropiolamide 1a (83 mg, $0.3 \mathrm{mmol})$, 3-Hydroxyadamantane 1- carboxylic acid $\mathbf{4 h}$ ( $112 \mathrm{mg}, 0.6$ $\mathrm{mmol}$ ) and $\mathrm{K}_{2} \mathrm{~S}_{2} \mathrm{O}_{8}(421 \mathrm{mg}, 1.5 \mathrm{mmol})$ in $5 \mathrm{~mL}$ of Acetonitrile: water (1:1), silver carbonate $(8.3 \mathrm{mg}$, $10 \mathrm{~mol} \%$ ) was added at room temperature. The reaction mixture was stirred at $70{ }^{\circ} \mathrm{C}$ for $7 \mathrm{~h}$. After completion of the reaction (monitored by TLC), the reaction mixture was concentrated under reduced pressure. The crude product was purified by column chromatography on silica gel (EtOAc-hexanes) to afford the corresponding product 3-(3-hydroxyadamantan-1-yl)-1-methyl-4-phenyl-1azaspiro[4.5]deca-3,6,9-triene-2,8-dione $\mathbf{5 h}, 97 \mathrm{mg}$, 80\% yield, yellow solid, $\mathrm{mp}: 103-105{ }^{\circ} \mathrm{C}$; $\mathrm{Rf}=$ 0.2 (Petroleum ether : EtOAc = 5:5 ); ${ }^{1} \mathrm{H}$ NMR $(300 \mathrm{MHz}, \mathrm{CDCl} 3) \delta 7.33-7.18(\mathrm{~m}, 4 \mathrm{H}), 6.96(\mathrm{~d}, J=$ $6.5 \mathrm{~Hz}, 2 \mathrm{H}), 6.45(\mathrm{~d}, J=10.1 \mathrm{~Hz}, 2 \mathrm{H}), 6.30(\mathrm{~d}, J=10.1 \mathrm{~Hz}, 2 \mathrm{H}), 2.87(\mathrm{~s}, 3 \mathrm{H}), 2.11(\mathrm{~s}, 2 \mathrm{H}), 1.82(\mathrm{~s}$, 2H), $1.76(\mathrm{~s}, 3 \mathrm{H}), 1.61(\mathrm{~s}, 5 \mathrm{H}), 1.54-1.46(\mathrm{~m}, 2 \mathrm{H}) ;{ }^{13} \mathrm{C}$ NMR (101 MHz, CDCl3) $\delta$ 184.1, 170.0, 148.5, 145.8, 143.4, 142.1, 133.1, 132.6, 129.3, 128.5, 127.3, 68.3, 67.8, 48.0, 44.1, 40.6, 39.2, 35.0, 30.4, 26.2; IR (KBr): vmax $=3414,2918,1676,1384,756 \mathrm{~cm}^{-1}$; HRMS (ESI) : m/z calcd for $\mathrm{C}_{26} \mathrm{H}_{28} \mathrm{NO}_{3}(\mathrm{M}+\mathrm{H})^{+}: 402.2069$, Found : 402.2082 .

\section{1-Phenyl-2-propionyl-6,7-dihydro-3H-pyrrolo[2,1-j]quinoline-3,9(5H)-dione (7a):}

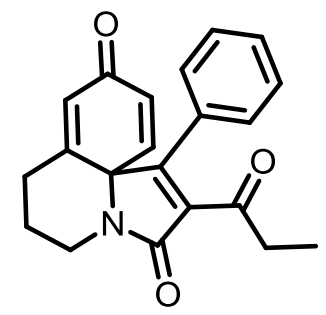

Following the general procedure, to the solution of 1-(6-methoxy-3,4-dihydroquinolin-1(2H)-yl)-3phenylprop-2-yn-1-one 6a (88 mg, $0.3 \mathrm{mmol}$ ), 2-oxobutanoic acid $\mathbf{2 b}$ ( $61 \mathrm{mg}, 0.6 \mathrm{mmol}$ ) and $\mathrm{K}_{2} \mathrm{~S}_{2} \mathrm{O}_{8}$ ( $\left.406 \mathrm{mg}, 1.5 \mathrm{mmol}\right)$ in $5 \mathrm{~mL}$ of Acetonitrile: water (1:1), silver carbonate $(8.3 \mathrm{mg}, 10 \mathrm{~mol}$ $\%$ ) was added at room temperature. The reaction mixture was stirred at $70{ }^{\circ} \mathrm{C}$ for $7 \mathrm{~h}$. After completion of the reaction (monitored by TLC), the reaction mixture was concentrated under reduced pressure. The crude product was purified by column chromatography on silica gel (EtOAc-hexanes) to afford the corresponding product 1-Phenyl-2-propionyl-6,7-dihydro-3H-pyrrolo[2,1-j]quinoline3,9(5H)-dione 7a, $74 \mathrm{mg}, 74 \%$ yield, yellow solid, $\mathrm{mp}: 138-140{ }^{\circ} \mathrm{C}, \mathrm{Rf}=0.2$ (Petroleum ether : EtOAc $=5: 5) ;{ }^{1} \mathrm{H}$ NMR $\left(400 \mathrm{MHz}, \mathrm{CDCl}_{3}\right) \delta 7.40-7.33(\mathrm{~m}, 1 \mathrm{H}), 7.33-7.27(\mathrm{~m}, 2 \mathrm{H}), 7.02-6.96$ 
$(\mathrm{m}, 2 \mathrm{H}), 6.54(\mathrm{~d}, J=9.8 \mathrm{~Hz}, 1 \mathrm{H}), 6.37(\mathrm{t}, J=1.5 \mathrm{~Hz}, 1 \mathrm{H}), 6.22(\mathrm{dd}, J=9.8,1.6 \mathrm{~Hz}, 1 \mathrm{H}), 4.32-4.12$ (m, 1H), $2.85-2.78(\mathrm{~m}, 1 \mathrm{H}), 2.77-2.67(\mathrm{~m}, 2 \mathrm{H}), 2.59-2.46(\mathrm{~m}, 2 \mathrm{H}), 2.17-2.04(\mathrm{~m}, 1 \mathrm{H}), 1.94-$ $1.82(\mathrm{~m}, 1 \mathrm{H}), 1.02(\mathrm{t}, J=7.2 \mathrm{~Hz}, 3 \mathrm{H}) ;{ }^{13} \mathrm{C} \mathrm{NMR}\left(125 \mathrm{MHz}, \mathrm{CDCl}_{3}\right) \delta 193.9,179.4,166.3,154.7$, $152.4,140.7,131.4,127.8,125.3,124.9,124.8,123.5,123.1,66.7,31.8,31.6,22.1,21.5,2.5$; IR $(\mathrm{KBr}): \operatorname{vmax}=2976,1698,1673,1376,1270,746 \mathrm{~cm}-1$; HRMS $(\mathrm{ESI}): \mathrm{m} / z$ calcd for $\mathrm{C}_{21} \mathrm{H}_{20} \mathrm{NO}_{3}$ $(\mathrm{M}+\mathrm{H})^{+}: 334.1443$, found: 334.1436 .

\section{2-(3-Methylbutanoyl)-1-phenyl-6,7-dihydro-3H-pyrrolo[2,1-j]quinoline-3,9(5H)-dione (7b):}

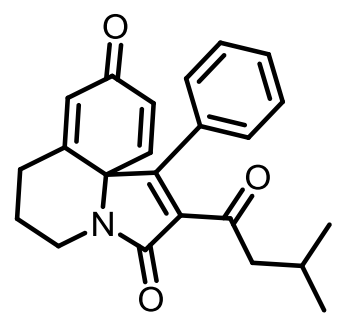

Following the general procedure, to the solution of 1-(6-methoxy-3,4-dihydroquinolin-1(2H)-yl)-3phenylprop-2-yn-1-one 6a ( $88 \mathrm{mg}, 0.3 \mathrm{mmol}$ ), 4-methyl-2-oxovaleric acid $\mathbf{2 d}$ ( $71 \mathrm{mg}, 0.6 \mathrm{mmol}$ ) and $\mathrm{K}_{2} \mathrm{~S}_{2} \mathrm{O}_{8}$ ( $\left.406 \mathrm{mg}, 1.5 \mathrm{mmol}\right)$ in $5 \mathrm{~mL}$ of Acetonitrile: water (1:1), silver carbonate ( $\left.8.3 \mathrm{mg}, 10 \mathrm{~mol} \%\right)$ was added at room temperature. The reaction mixture was stirred at $70{ }^{\circ} \mathrm{C}$ for $7 \mathrm{~h}$. After completion of the reaction (monitored by TLC), the reaction mixture was concentrated under reduced pressure. The crude product was purified by column chromatography on silica gel (EtOAc-hexanes) to afford the corresponding product 2-(3-methylbutanoyl)-1-phenyl-6,7-dihydro-3H-pyrrolo[2,1-j]quinoline3,9(5H)-dione 7b, $85 \mathrm{mg}, 78 \%$ yield, pale yellow solid, mp: $142-144{ }^{\circ} \mathrm{C}, \mathrm{R}_{\mathrm{f}}=0.2$ (Petroleum ether : EtOAc $=5: 5) ;{ }^{1} \mathrm{H}$ NMR $\left(400 \mathrm{MHz}, \mathrm{CDCl}_{3}\right) \delta 7.40-7.33(\mathrm{~m}, 1 \mathrm{H}), 7.33-7.26(\mathrm{~m}, 2 \mathrm{H}), 7.03-6.96$ $(\mathrm{m}, 2 \mathrm{H}), 6.55(\mathrm{~d}, J=9.8 \mathrm{~Hz}, 1 \mathrm{H}), 6.38(\mathrm{t}, J=1.4 \mathrm{~Hz}, 1 \mathrm{H}), 6.21(\mathrm{dd}, J=9.8,1.6 \mathrm{~Hz}, 1 \mathrm{H}), 4.30-4.15$ (m, 1H), $2.90-2.72(\mathrm{~m}, 1 \mathrm{H}), 2.57(\mathrm{~d}, J=6.8 \mathrm{~Hz}, 2 \mathrm{H}), 2.56-2.46(\mathrm{~m}, 2 \mathrm{H}), 2.15-2.01(\mathrm{~m}, 2 \mathrm{H}), 1.96$ $-1.79(\mathrm{~m}, 1 \mathrm{H}), 0.84(\mathrm{dd}, J=6.7,1.5 \mathrm{~Hz}, 6 \mathrm{H}) ;{ }^{13} \mathrm{C} \mathrm{NMR}\left(100 \mathrm{MHz}, \mathrm{CDCl}_{3}\right) \delta 198.1,184.2,171.0$, $159.1,157.2$, 145.5, 136.6, 132.5, 130.1, 129.5, 128.2, 128.0, 71.4, 51.8, 36.5, 26.8, 26.3, 24.3, 22.5, 22.4; IR (KBr):vmax $=2956,1701,1377,1275,702 \mathrm{~cm}-1$; HRMS (ESI): $\mathrm{m} / z$ calcd for $\mathrm{C}_{23} \mathrm{H}_{24} \mathrm{NO}_{3}$ $(\mathrm{M}+\mathrm{H})^{+}: 362.1756$, found: 362.1762 .

2-Benzoyl-1-phenyl-6,7-dihydro-3H-pyrrolo[2,1-j]quinoline-3,9(5H)-dione (7c):

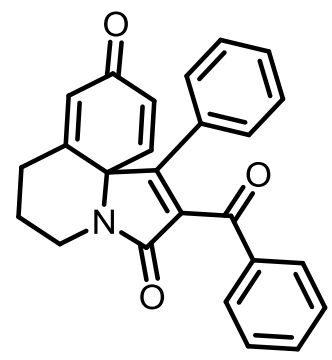


Following the general procedure, to the solution of 1-(6-methoxy-3,4-dihydroquinolin-1(2H)-yl)-3phenylprop-2-yn-1-one 6a (88 mg, $0.3 \mathrm{mmol})$, 2-oxo-2-phenylacetic acid $2 \mathbf{e}$ ( $92 \mathrm{mg}, 0.6 \mathrm{mmol}$ ) and $\mathrm{K}_{2} \mathrm{~S}_{2} \mathrm{O}_{8}$ ( $\left.406 \mathrm{mg}, 1.5 \mathrm{mmol}\right)$ in $5 \mathrm{~mL}$ of Acetonitrile: water (1:1), silver carbonate (8.3 mg, $10 \mathrm{~mol}$ $\%$ ) was added at room temperature. The reaction mixture was stirred at $70{ }^{\circ} \mathrm{C}$ for $7 \mathrm{~h}$. After completion of the reaction (monitored by TLC), the reaction mixture was concentrated under reduced pressure. The crude product was purified by column chromatography on silica gel (EtOAc-hexanes) to afford the corresponding product 2-benzoyl-1-phenyl-6,7-dihydro-3H-pyrrolo[2,1-j]quinoline-3,9(5H)-dione 7c, $86 \mathrm{mg}, 75 \%$ yield, yellow solid, mp:183-185 ${ }^{\circ} \mathrm{C}, \mathrm{R}_{\mathrm{f}}=0.2$ (Petroleum ether : EtOAc $=5: 5$ ); ${ }^{1} \mathrm{H}$ NMR (400 MHz, $\left.\mathrm{CDCl}_{3}\right) \delta 7.82-7.77(\mathrm{~m}, 2 \mathrm{H}), 7.56-7.50(\mathrm{~m}, 1 \mathrm{H}), 7.41-7.36(\mathrm{~m}, 2 \mathrm{H}), 7.25-7.22$ (m, 1H), $7.20-7.14(\mathrm{~m}, 2 \mathrm{H}), 7.09-7.04(\mathrm{~m}, 2 \mathrm{H}), 6.66(\mathrm{~d}, J=9.8 \mathrm{~Hz}, 1 \mathrm{H}), 6.48(\mathrm{t}, J=1.5 \mathrm{~Hz}, 1 \mathrm{H})$, $6.26(\mathrm{dd}, J=9.8,1.6 \mathrm{~Hz}, 1 \mathrm{H}), 4.24(\mathrm{dd}, J=14.1,8.0 \mathrm{~Hz}, 1 \mathrm{H}), 2.93-2.78(\mathrm{~m}, 1 \mathrm{H}), 2.68-2.53(\mathrm{~m}$, $2 \mathrm{H}), 2.38-2.24(\mathrm{~m}, 1 \mathrm{H}), 2.01-1.86(\mathrm{~m}, 1 \mathrm{H}) ;{ }^{13} \mathrm{C} \mathrm{NMR}\left(100 \mathrm{MHz}, \mathrm{CDCl}_{3}\right) \delta 190.6,184.4,171.1$, 157.7, 156.8, 146.1, 137.1, 135.7, 134.1, 132.3, 130.3, 129.7, 129.4, 128.7, 128.5, 127.9, 73.7, 36.6, 27.0, 26.6; IR (KBr): vmax $=2946,1701,1665,1332,899,755 \mathrm{~cm}^{-}{ }^{1}$; HRMS (ESI): $\mathrm{m} / z$ calcd for $\mathrm{C}_{25} \mathrm{H}_{20} \mathrm{NO}_{3}(\mathrm{M}+\mathrm{H})^{+}:$382.1443, found: 382.1438 .

\section{1-(4-Chlorophenyl)-2-propionyl-6,7-dihydro-3H-pyrrolo[2,1-j]quinoline-3,9(5H)-dione (7d) :}

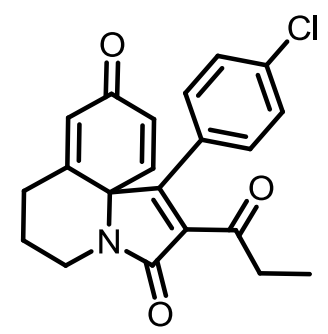

Following the general procedure, to the solution of 3-(4-chlorophenyl)-1-(6-methoxy-3,4dihydroquinolin-1(2H)-yl)prop-2-yn-1-one $\mathbf{6 b}(99 \mathrm{mg}, 0.3 \mathrm{mmol})$, 2-oxobutanoic acid $2 \mathbf{b}$ ( $61 \mathrm{mg}, 0.6$ mmol) and $\mathrm{K}_{2} \mathrm{~S}_{2} \mathrm{O}_{8}(409 \mathrm{mg}, 1.5 \mathrm{mmol})$ in $5 \mathrm{~mL}$ of Acetonitrile: water (1:1), silver carbonate (8.3 mg, $10 \mathrm{~mol} \%$ ) was added at room temperature. The reaction mixture was stirred at $70{ }^{\circ} \mathrm{C}$ for $7 \mathrm{~h}$. After completion of the reaction (monitored by TLC), the reaction mixture was concentrated under reduced pressure. The crude product was purified by column chromatography on silica gel (EtOAc-hexanes) to afford the corresponding product 1-(4-chlorophenyl)-2-propionyl-6,7-dihydro-3H-pyrrolo[2,1j]quinoline-3,9(5H)-dione 7d, $84 \mathrm{mg}, 76 \%$ yield, yellow solid, mp: $115-117{ }^{\circ} \mathrm{C}, \mathrm{R}_{f}=0.2$ (Petroleum ether : EtOAc = 5:5 ); ${ }^{1} \mathrm{H}$ NMR $\left(500 \mathrm{MHz}, \mathrm{CDCl}_{3}\right) \delta 7.30-7.28(\mathrm{~m}, 1 \mathrm{H}), 7.27(\mathrm{~d}, J=1.9 \mathrm{~Hz}, 1 \mathrm{H})$, $6.95-6.93(\mathrm{~m}, 1 \mathrm{H}), 6.93-6.92(\mathrm{~m}, 1 \mathrm{H}), 6.52(\mathrm{~d}, J=9.8 \mathrm{~Hz}, 1 \mathrm{H}), 6.38(\mathrm{~d}, J=1.4 \mathrm{~Hz}, 1 \mathrm{H}), 6.25(\mathrm{dd}$, $J=9.8,1.6 \mathrm{~Hz}, 1 \mathrm{H}), 4.21(\mathrm{dd}, J=13.6,8.7 \mathrm{~Hz}, 1 \mathrm{H}), 2.91-2.75(\mathrm{~m}, 3 \mathrm{H}), 2.52(\mathrm{~m}, 2 \mathrm{H}), 2.05(\mathrm{~m}, 1 \mathrm{H})$, $1.93-1.83(\mathrm{~m}, 1 \mathrm{H}), 1.04(\mathrm{t}, J=7.2 \mathrm{~Hz}, 3 \mathrm{H}) ;{ }^{13} \mathrm{C} \mathrm{NMR}\left(100 \mathrm{MHz}, \mathrm{CDCl}_{3}\right) \delta 198.4,184.0,170.8$, 158.6, 157.0, 145.1, 136.4, 136.3, 132.7, 129.6, 129.3, 128.6, 128.0, 71.3, 36.5, 36.4, 26.8, 26.3, 7.2; IR (KBr):vmax = 2944, 1701, 1381, 1161, 1021, $757 \mathrm{~cm}^{-1}$; HRMS (ESI): $\mathrm{m} / z$ calcd for $\mathrm{C}_{21} \mathrm{H}_{19} \mathrm{NO}_{3} \mathrm{Cl}$ $(\mathrm{M}+\mathrm{H})^{+}:$368.1045, found: 368.1053 . 

dione(7e):

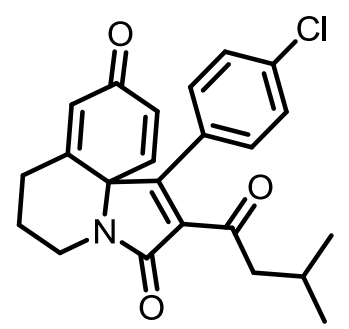

Following the general procedure, to the solution of 3-(4-chlorophenyl)-1-(6-methoxy-3,4dihydroquinolin-1(2H)-yl)prop-2-yn-1-one $\mathbf{6 b}$ (99 mg, $0.3 \mathrm{mmol}$ ), 4-methyl-2-oxovaleric acid $2 \mathbf{d}$ ( 71 $\mathrm{mg}, 0.6 \mathrm{mmol}$ ) and $\mathrm{K}_{2} \mathrm{~S}_{2} \mathrm{O}_{8}(409 \mathrm{mg}, 1.5 \mathrm{mmol})$ in $5 \mathrm{~mL}$ of Acetonitrile: water (1:1), silver carbonate $(8.3 \mathrm{mg}, 10 \mathrm{~mol} \%)$ was added at room temperature. The reaction mixture was stirred at $70{ }^{\circ} \mathrm{C}$ for $7 \mathrm{~h}$. After completion of the reaction (monitored by TLC), the reaction mixture was concentrated under reduced pressure. The crude product was purified by column chromatography on silica gel (EtOAchexanes) to afford the corresponding product 1-(4-chlorophenyl)-2-(3-methylbutanoyl)-6,7-dihydro3H-pyrrolo[2,1-j]quinoline-3,9(5H)-dione 7e, $94 \mathrm{mg}, 79 \%$ yield, yellow solid, mp: $110-112{ }^{\circ} \mathrm{C}, \mathrm{Rf}=$ 0.2 (Petroleum ether : EtOAc $=5: 5) ;{ }^{1} \mathrm{H}$ NMR $\left(400 \mathrm{MHz}, \mathrm{CDCl}_{3}\right) \delta 7.23-7.19(\mathrm{~m}, 2 \mathrm{H}), 6.89-6.84$ (m, 2H), 6.45 (d, $J=9.8 \mathrm{~Hz}, 1 \mathrm{H}), 6.31(\mathrm{t}, J=1.5 \mathrm{~Hz}, 1 \mathrm{H}), 6.17$ (dd, $J=9.8,1.6 \mathrm{~Hz}, 1 \mathrm{H}), 4.14$ (ddd, $J$ $=14.1,9.2,1.2 \mathrm{~Hz}, 1 \mathrm{H}), 2.78-2.69(\mathrm{~m}, 1 \mathrm{H}), 2.65-2.54(\mathrm{~m}, 2 \mathrm{H}), 2.51-2.39(\mathrm{~m}, 2 \mathrm{H}), 2.07-1.94$ $(\mathrm{m}, 2 \mathrm{H}), 1.86-1.76(\mathrm{~m}, 1 \mathrm{H}), 0.80(\mathrm{dd}, J=6.7,2.7 \mathrm{~Hz}, 6 \mathrm{H}) ;{ }^{13} \mathrm{C} \mathrm{NMR}\left(100 \mathrm{MHz}, \mathrm{CDCl}_{3}\right) \delta 197.8$, 184.0, 170.8, 158.2, 157.0, 145.2, 136.8, 136.4, 132.7, 129.6, 129.4, 128.6, 127.9, 71.3, 51.7, 36.5, 26.8, 26.3, 24.3, 22.5, 22.4; IR (KBr):vmax= 2956, 1699, 1377, 1274, 849, $757 \mathrm{~cm}^{-1}$; HRMS (ESI): $\mathrm{m} / \mathrm{z}$ calcd for $\mathrm{C}_{23} \mathrm{H}_{23} \mathrm{NO}_{3} \mathrm{Cl}(\mathrm{M}+\mathrm{H})+:$ 396.1366, found: 396.1382 .

2-Benzoyl-1-(4-chlorophenyl)-6,7-dihydro-3H-pyrrolo[2,1-j]quinoline-3,9(5H)-dione (7f):

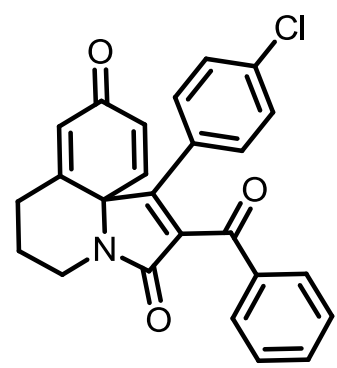

Following the general procedure, to the solution of 3-(4-chlorophenyl)-1-(6-methoxy-3,4dihydroquinolin-1(2H)-yl)prop-2-yn-1-one $\mathbf{6 b}$ (99 mg, $0.3 \mathrm{mmol}), \quad 2$-oxo-2-phenylacetic acid $2 \mathbf{e}$ ( 92 $\mathrm{mg}, 0.6 \mathrm{mmol}$ ) and $\mathrm{K}_{2} \mathrm{~S}_{2} \mathrm{O}_{8}$ ( $406 \mathrm{mg}, 1.5 \mathrm{mmol}$ ) in $5 \mathrm{~mL}$ of Acetonitrile: water (1:1), silver carbonate $(8.3 \mathrm{mg}, 10 \mathrm{~mol} \%)$ was added at room temperature. The reaction mixture was stirred at $70{ }^{\circ} \mathrm{C}$ for $9 \mathrm{~h}$. After completion of the reaction (monitored by TLC), the reaction mixture was concentrated under 
reduced pressure. The crude product was purified by column chromatography on silica gel (EtOAchexanes) to afford the corresponding product 2-benzoyl-1-(4-chlorophenyl)-6,7-dihydro-3Hpyrrolo[2,1-j]quinoline-3,9(5H)-dione 7f, $94 \mathrm{mg}, 75 \%$ yield, Pale yellow solid, mp:103- $105{ }^{\circ} \mathrm{C}, \mathrm{Rf}=$ 0.2 (Petroleum ether : EtOAc $=5: 5) ;{ }^{1} \mathrm{H}$ NMR $\left(400 \mathrm{MHz}, \mathrm{CDCl}_{3}\right) \delta 7.80(\mathrm{dt}, J=8.5,1.5 \mathrm{~Hz}, 2 \mathrm{H})$, $7.60-7.53(\mathrm{~m}, 1 \mathrm{H}), 7.46-7.38(\mathrm{~m}, 2 \mathrm{H}), 7.21-7.13(\mathrm{~m}, 2 \mathrm{H}), 7.06-6.97(\mathrm{~m}, 2 \mathrm{H}), 6.65(\mathrm{~d}, J=9.8$ Hz, 1H), 6.49 (t, $J=1.5 \mathrm{~Hz}, 1 \mathrm{H}), 6.29$ (dd, $J=9.8,1.6 \mathrm{~Hz}, 1 \mathrm{H}), 4.27-4.17(\mathrm{~m}, 1 \mathrm{H}), 2.90-2.76(\mathrm{~m}$, $1 \mathrm{H}), 2.65-2.53(\mathrm{~m}, 2 \mathrm{H}), 2.31-2.21(\mathrm{~m}, 1 \mathrm{H}), 1.98-1.87(\mathrm{~m}, 1 \mathrm{H}) ;{ }^{13} \mathrm{C} \mathrm{NMR}\left(100 \mathrm{MHz}, \mathrm{CDCl}_{3}\right) \delta$ 190.4, 184.2, 170.8, 157.6, 155.4, 145.9, 137.7, 136.7, 135.5, 134.4, 132.4, 129.8, 129.4, 129.2, 128.9, 128.8, 128.1, 71.4, 36.6, 27.0, 26.6; IR (KBr):vmax= 2962, 1701, 1659, 1377, 1091, $751 \mathrm{~cm}^{-1}$; HRMS (ESI): $m / z$ calcd for $\mathrm{C}_{25} \mathrm{H}_{19} \mathrm{NO}_{3} \mathrm{Cl}(\mathrm{M}+\mathrm{H})^{+}: 416.1053$, found: 416.1046 .

\section{1-(4-Acetylphenyl)-2-propionyl-6,7-dihydro-3H-pyrrolo[2,1-j]quinoline-3,9(5H)-dione} (7g):

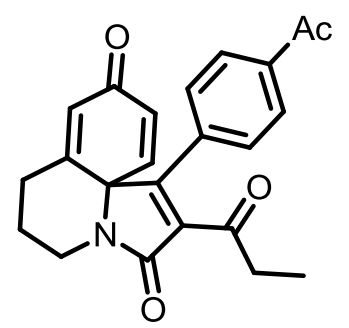

Following the general procedure, to the solution of 3-(4-acetylphenyl)-1-(6-methoxy-3,4dihydroquinolin-1(2H)-yl)prop-2-yn-1-one $\mathbf{6 c}$ (100 mg, $0.3 \mathrm{mmol}$ ), 2-oxobutanoic acid $2 \mathbf{b}$ ( $61 \mathrm{mg}, 0.6$ mmol) and $\mathrm{K}_{2} \mathrm{~S}_{2} \mathrm{O}_{8}$ ( $\left.403 \mathrm{mg}, 1.5 \mathrm{mmol}\right)$ in $5 \mathrm{~mL}$ of Acetonitrile: water (1:1), silver carbonate (8.3 $\mathrm{mg}, 10 \mathrm{~mol} \%$ ) was added at room temperature. The reaction mixture was stirred at $70{ }^{\circ} \mathrm{C}$ for $7 \mathrm{~h}$. After completion of the reaction (monitored by TLC), the reaction mixture was concentrated under reduced pressure. The crude product was purified by column chromatography on silica gel (EtOAc-hexanes) to afford the corresponding product 1-(4-acetylphenyl)-2-propionyl-6,7-dihydro-3H-pyrrolo[2,1j]quinoline-3,9(5H)-dione 7g, $78 \mathrm{mg}$. $69 \%$ yield, light brown liquid, $\mathrm{Rf}=0.2$ (Petroleum ether : EtOAc $=5: 5) ;{ }^{1} \mathrm{H}$ NMR $\left(400 \mathrm{MHz}, \mathrm{CDCl}_{3}\right) \delta 7.88(\mathrm{~d}, J=8.4 \mathrm{~Hz}, 2 \mathrm{H}), 7.07(\mathrm{~d}, J=8.4 \mathrm{~Hz}, 2 \mathrm{H}), 6.56$ $(\mathrm{d}, J=9.8 \mathrm{~Hz}, 1 \mathrm{H}), 6.37(\mathrm{t}, J=1.3 \mathrm{~Hz}, 1 \mathrm{H}), 6.24(\mathrm{dd}, J=9.8,1.6 \mathrm{~Hz}, 1 \mathrm{H}), 4.23(\mathrm{dd}, J=13.7,8.7 \mathrm{~Hz}$, $1 \mathrm{H}), 3.06-2.75(\mathrm{~m}, 4 \mathrm{H}), 2.58(\mathrm{~s}, 3 \mathrm{H}), 2.57-2.48(\mathrm{~m}, 2 \mathrm{H}), 2.18-2.01(\mathrm{~m}, 1 \mathrm{H}), 1.97-1.82(\mathrm{~m}, 1 \mathrm{H})$, $1.04(\mathrm{t}, J=7.2 \mathrm{~Hz}, 2 \mathrm{H}) ;{ }^{13} \mathrm{C} \mathrm{NMR}\left(100 \mathrm{MHz}, \mathrm{CDCl}_{3}\right) \delta 198.0,197.0,183.8,170.7,159.2,156.9$, $144.8,137.7,136.2,134.3,132.8,129.6,128.3,128.0,71.3,36.5,36.3,26.8,26.6,26.3,7.2$; IR $(\mathrm{KBr}): \operatorname{vmax}=3506,2959,1694,1376,1039,759 \mathrm{~cm}-1 ; \mathrm{IR}(\mathrm{KBr}): \operatorname{vmax}=2929,1681$, $1453,1373,1267,759 \mathrm{~cm}^{-1}$; HRMS (ESI): $\mathrm{m} / \mathrm{z}$ calcd for $\mathrm{C}_{23} \mathrm{H}_{22} \mathrm{NO}_{4}(\mathrm{M}+\mathrm{H})^{+}: 376.1549$, found: 376.1545 .

1-(4-Acetylphenyl)-2-(3-methylbutanoyl)-6,7-dihydro-3H-pyrrolo[2,1-j]quinoline3,9(5H)-dione (7h): 


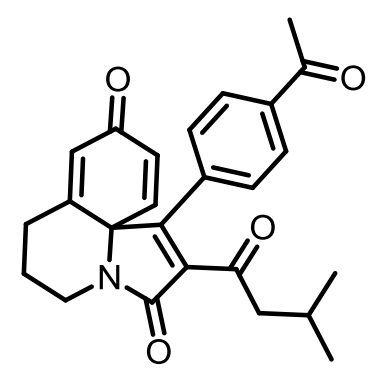

Following the general procedure, to the solution of 1-(4-acetylphenyl)-1-(6-methoxy-3,4dihydroquinolin-1(2H)-yl)prop-2-yn-1-one $\mathbf{6 c}(100 \mathrm{mg}, 0.3 \mathrm{mmol})$ and 4-methyl-2-oxovaleric acid 2d ( $71 \mathrm{mg}, 0.6 \mathrm{mmol}$ ) and $\mathrm{K}_{2} \mathrm{~S}_{2} \mathrm{O}_{8}(403 \mathrm{mg}, 1.5 \mathrm{mmol})$ in $5 \mathrm{~mL}$ of Acetonitrile: water (1:1), silver carbonate $(8.3 \mathrm{mg}, 10 \mathrm{~mol} \%)$ was added at room temperature. The reaction mixture was stirred at $70{ }^{\circ} \mathrm{C}$ for $7 \mathrm{~h}$. After completion of the reaction (monitored by TLC), the reaction mixture was concentrated under reduced pressure. The crude product was purified by column chromatography on silica gel (EtOAc-hexanes) to afford the corresponding product 1-(4-acetylphenyl)-2-(3-methylbutanoyl)-6,7dihydro-3H-pyrrolo[2,1-j]quinoline-3,9(5H)-dione $7 \mathbf{h}, 86 \mathrm{mg}, 71 \%$ yield, yellow oil, $\mathrm{Rf}=0.2$ (Petroleum ether : EtOAc = 5:5 ); ${ }^{1} \mathrm{H}$ NMR $\left(400 \mathrm{MHz}, \mathrm{CDCl}_{3}\right) \delta 7.91-7.85(\mathrm{~m}, 2 \mathrm{H}), 7.11-7.05(\mathrm{~m}$, 2H), $6.57(\mathrm{~d}, J=9.8 \mathrm{~Hz}, 1 \mathrm{H}), 6.37(\mathrm{t}, J=1.4 \mathrm{~Hz}, 1 \mathrm{H}), 6.24(\mathrm{dd}, J=9.8,1.6 \mathrm{~Hz}, 1 \mathrm{H}), 4.23(\mathrm{dd}, J=$ 13.7, $8.6 \mathrm{~Hz}, 1 \mathrm{H}), 2.88-2.78(\mathrm{~m}, 1 \mathrm{H}), 2.73-2.69(\mathrm{~m}, 2 \mathrm{H}), 2.60-2.48(\mathrm{~m}, 5 \mathrm{H}), 2.15-2.04(\mathrm{~m}, 2 \mathrm{H})$, $1.95-1.84(\mathrm{~m}, 1 \mathrm{H}), 0.87(\mathrm{dd}, J=6.7,2.1 \mathrm{~Hz}, 6 \mathrm{H}) ;{ }^{13} \mathrm{C} \mathrm{NMR}\left(100 \mathrm{MHz}, \mathrm{CDCl}_{3}\right) \delta 197.4,197.0$, 183.9, 170.6, 158.9, 156.9, 144.9, 137.7, 136.7, 134.2, 132.8, 129.6, 128.4, 127.9, 71.2, 51.6, 36.5, 26.8, 26.6, 26.3, 24.3, 22.5, 22.4; IR (KBr): vmax=2957, 1678, 1454,1372, 1021, $755 \mathrm{~cm}^{-1}$; HRMS (ESI): $m / z$ calcd for $\mathrm{C}_{25} \mathrm{H}_{26} \mathrm{NO}_{4}(\mathrm{M}+\mathrm{H})^{+}: 404.1862$, found: 404.1856 .

\section{1-(4-Acetylphenyl)-2-benzoyl-6,7-dihydro-3H-pyrrolo[2,1-j]quinoline-3,9(5H)-dione} (7i):

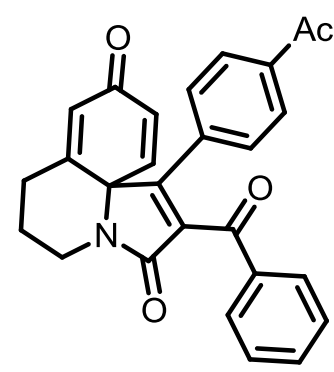

Following the general procedure, to the solution of 3-(4-acetylphenyl)-1-(6-methoxy-3,4dihydroquinolin-1(2H)-yl)prop-2-yn-1-one $\mathbf{6 c}(100 \mathrm{mg}, 0.3 \mathrm{mmol})$ and 2-oxo-2-phenylacetic acid 2e ( $92 \mathrm{mg}, 0.6 \mathrm{mmol})$ in $5 \mathrm{~mL}$ of Acetonitrile: water (1:1), silver carbonate $(8.3 \mathrm{mg}, 10 \mathrm{~mol} \%)$ was added at room temperature. The reaction mixture was stirred at $70{ }^{\circ} \mathrm{C}$ for $9 \mathrm{~h}$. After completion of the reaction 
(monitored by TLC), the reaction mixture was concentrated under reduced pressure. The crude product was purified by column chromatography on silica gel (EtOAc-hexanes) to afford the corresponding product 1-(4-acetylphenyl)-2-benzoyl-6,7-dihydro-3H-pyrrolo[2,1-j]quinoline-3,9(5H)dione 7i, $86 \mathrm{mg}, 70 \%$ yield, yellow oil, $\mathrm{Rf}=0.2$ (Petroleum ether : EtOAc $=5: 5) ;{ }^{1} \mathrm{H}$ NMR $(400$ $\left.\mathrm{MHz}, \mathrm{CDCl}_{3}\right) \delta 7.85-7.70(\mathrm{~m}, 4 \mathrm{H}), 7.62-7.50(\mathrm{~m}, 1 \mathrm{H}), 7.48-7.36(\mathrm{~m}, 2 \mathrm{H}), 7.24-7.10(\mathrm{~m}, 2 \mathrm{H})$, $6.68(\mathrm{~d}, J=9.8 \mathrm{~Hz}, 1 \mathrm{H}), 6.48(\mathrm{t}, J=1.4 \mathrm{~Hz}, 1 \mathrm{H}), 6.28(\mathrm{dd}, J=9.8,1.6 \mathrm{~Hz}, 1 \mathrm{H}), 4.35-4.16(\mathrm{~m}, 1 \mathrm{H})$, $2.93-2.80(\mathrm{~m}, 1 \mathrm{H}), 2.68-2.54(\mathrm{~m}, 2 \mathrm{H}), 2.50(\mathrm{~s}, 3 \mathrm{H}), 2.37-2.22(\mathrm{~m}, 1 \mathrm{H}), 2.02-1.85(\mathrm{~m}, 1 \mathrm{H}) ;{ }^{13} \mathrm{C}$ NMR $\left(126 \mathrm{MHz}, \mathrm{CDCl}_{3}\right) \delta 196.9,190.2,184.1,170.7,157.5,155.7,145.6,138.2,137.9,135.5$, 134.5, 134.1, 132.6, 129.8, 129.4, 128.8, 128.4, 128.3, 71.4, 36.6, 27.0, 26.6, 26.6; IR (KBr):vmax= 2927, 1669, 1601, 1329, 1264,748 $\mathrm{cm}^{-1}$; HRMS (ESI): $\mathrm{m} / \mathrm{z}$ calcd for $\mathrm{C}_{27} \mathrm{H}_{22} \mathrm{NO}_{4}(\mathrm{M}+\mathrm{H})^{+}: 424.1549$, found: 424.1544.

\section{2-(Tert-butyl)-1-phenyl-6,7-dihydro-3H-pyrrolo[2,1-j]quinoline-3,9(5H)-dione (7j):}

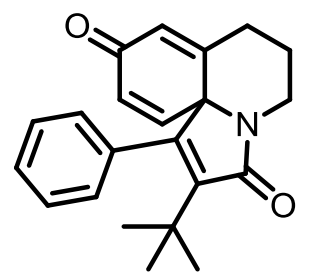

Following the general procedure, to the solution of 1-(6-methoxy-3,4-dihydroquinolin-1(2H)-yl)-3phenylprop-2-yn-1-one 6a (90 mg, $0.3 \mathrm{mmol}$ ), Pivalic acid $4 \mathbf{d}$ ( $70 \mathrm{mg}, 0.6 \mathrm{mmol}$ ) and $\mathrm{K}_{2} \mathrm{~S}_{2} \mathrm{O}_{8}(415 \mathrm{mg}$, $1.5 \mathrm{mmol})$ in $5 \mathrm{~mL}$ of Acetonitrile: water (1:1), silver carbonate $(8.3 \mathrm{mg}, 10 \mathrm{~mol} \%)$ was added at room temperature. The reaction mixture was stirred at $70{ }^{\circ} \mathrm{C}$ for $7 \mathrm{~h}$. After completion of the reaction (monitored by TLC), the reaction mixture was concentrated under reduced pressure. The crude product was purified by column chromatography on silica gel (EtOAc-hexanes) to afford the corresponding product 2-(tert-butyl)-1-phenyl-6,7-dihydro-3H-pyrrolo[2,1-j]quinoline-3,9(5H)-dione (7j), $97 \mathrm{mg}, 85 \%$ yield, Yellow Oil, Rf $=0.3$ (Petroleum ether : EtOAc $=7: 3$ ); ${ }^{1} \mathrm{H}$ NMR $(400 \mathrm{MHz}$, $\mathrm{CDCl} 3) \delta 7.25-7.24(\mathrm{~m}, 1 \mathrm{H}), 7.22-7.16(\mathrm{~m}, 2 \mathrm{H}), 6.91(\mathrm{~d}, \mathrm{~J}=6.6 \mathrm{~Hz}, 1 \mathrm{H}), 6.72(\mathrm{~d}, \mathrm{~J}=7.0 \mathrm{~Hz}, 1 \mathrm{H})$, $6.58(\mathrm{~d}, \mathrm{~J}=9.7 \mathrm{~Hz}, 1 \mathrm{H}), 6.21-6.09(\mathrm{~m}, 2 \mathrm{H}), 4.23-4.10(\mathrm{~m}, 1 \mathrm{H}), 2.81-2.69(\mathrm{~m}, \mathrm{~J}=13.9,10.1,7.9$ $\mathrm{Hz}, 1 \mathrm{H}), 2.57-2.44(\mathrm{~m}, 1 \mathrm{H}), 2.41-2.31(\mathrm{~m}, \mathrm{~J}=13.1,8.0 \mathrm{~Hz}, 1 \mathrm{H}), 2.05-1.95(\mathrm{~m}, 1 \mathrm{H}), 1.87-1.75$ $(\mathrm{m}, 1 \mathrm{H}), 1.14(\mathrm{~s}, 9 \mathrm{H}) ;{ }^{13} \mathrm{C} \mathrm{NMR}\left(101 \mathrm{MHz}, \mathrm{CDCl}_{3}\right) \delta 184.6,174.7,159.5,149.1,146.9,144.4,132.3$, 131.6, 130.3, 130.1, 129.1, 128.4, 128.3, 127.2, 127.0, 71.6, 36.5, 34.4, 29.7, 27.1, 26.0; IR (KBr): vmax $=3506,2959,1694,1376,1039,759 \mathrm{~cm}^{-1}$; HRMS (ESI) : m/z calcd for $\mathrm{C}_{22} \mathrm{H}_{24} \mathrm{NO}_{2}(\mathrm{M}+\mathrm{H})^{+}$: 334.1807, Found : 334.1805 .

\section{Gram scale reaction procedure for $3 \mathrm{~b}$ :}

To the solution of 3-(3,5-dimethylphenyl)-N-(4-methoxyphenyl)-N-methylpropiolamide $\mathbf{1 b}$ (1 g, $3.41 \mathrm{mmol}$ ), Pyruvic acid 2a ( $0.6 \mathrm{~g}, 6.8 \mathrm{mmol})$ and $\mathrm{K}_{2} \mathrm{~S}_{2} \mathrm{O}_{8}(4.5 \mathrm{~g}, 17.05 \mathrm{mmol})$ in $20 \mathrm{~mL}$ of Acetonitrile: water (9:1), silver carbonate $(93 \mathrm{mg}, 10 \mathrm{~mol} \%)$ was added at room temperature. 
The reaction mixture was stirred at $70{ }^{\circ} \mathrm{C}$ for $7 \mathrm{~h}$. After completion of the reaction (monitored by TLC), the reaction mixture was concentrated under reduced pressure. The crude product was purified by column chromatography on silica gel (EtOAc-hexanes) to afford the corresponding product 3-acetyl-4-(3,5-dimethylphenyl)-1-methyl-1-azaspiro[4.5]deca-3,6,9triene-2,8-dione $\mathbf{3 b}, 0.89 \mathrm{~g}, 81 \%$ yield, off-white solid.

\section{References:}

1] (a) Liu, Y.; Wang, Q.-L.; Zhou, C.-Z.; Xiong, B.-Q.; Zhang, P.-L.; Yang, C.-A.; Tang, K.-W. J. Org. Chem. 2018, 83, 2210-2218. (b) Jin, D.-P.; Gao, P.; Chen, D.-Q.; Chen, S.; Wang, J.; Liu, X.-Y.; Liang, Y. -M. Org. Lett. 2016, 18, 3486-3489. (c) Beinart, L.L.; Sun, X.; Sidman, E.; Kesharwani, T. Tetrahedron Lett. 2013, 54, 1344-1347. 
6. ${ }^{1} \mathrm{H}$ NMR and ${ }^{13} \mathrm{C}$ NMR and ${ }^{19}$ FNMR Spectral Copies of compounds

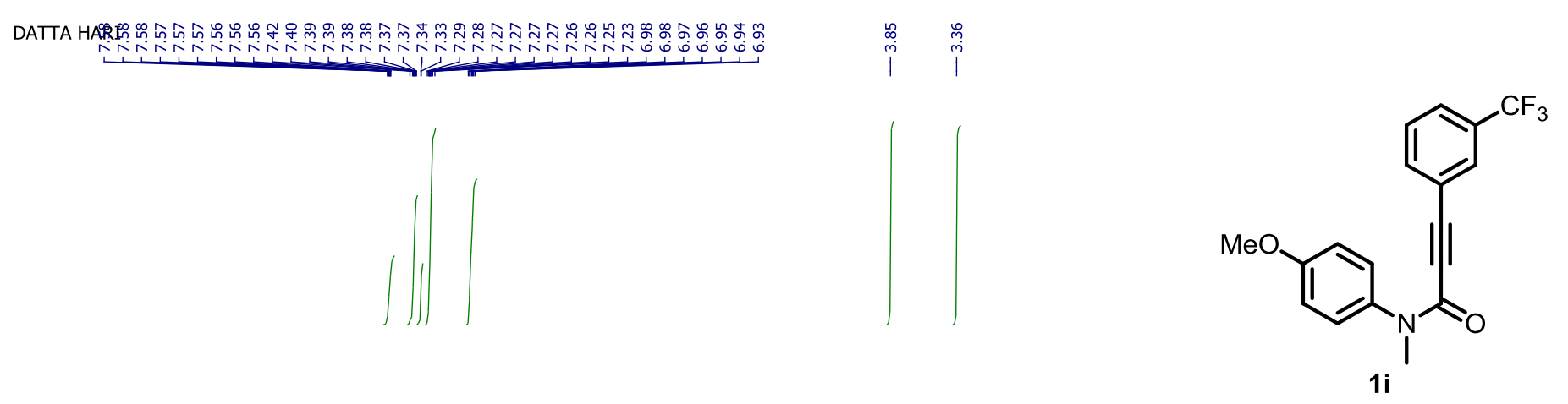

${ }^{1} \mathrm{H} \mathrm{NMR,} \mathrm{CDCl}_{3}, 400 \mathrm{MHz}$

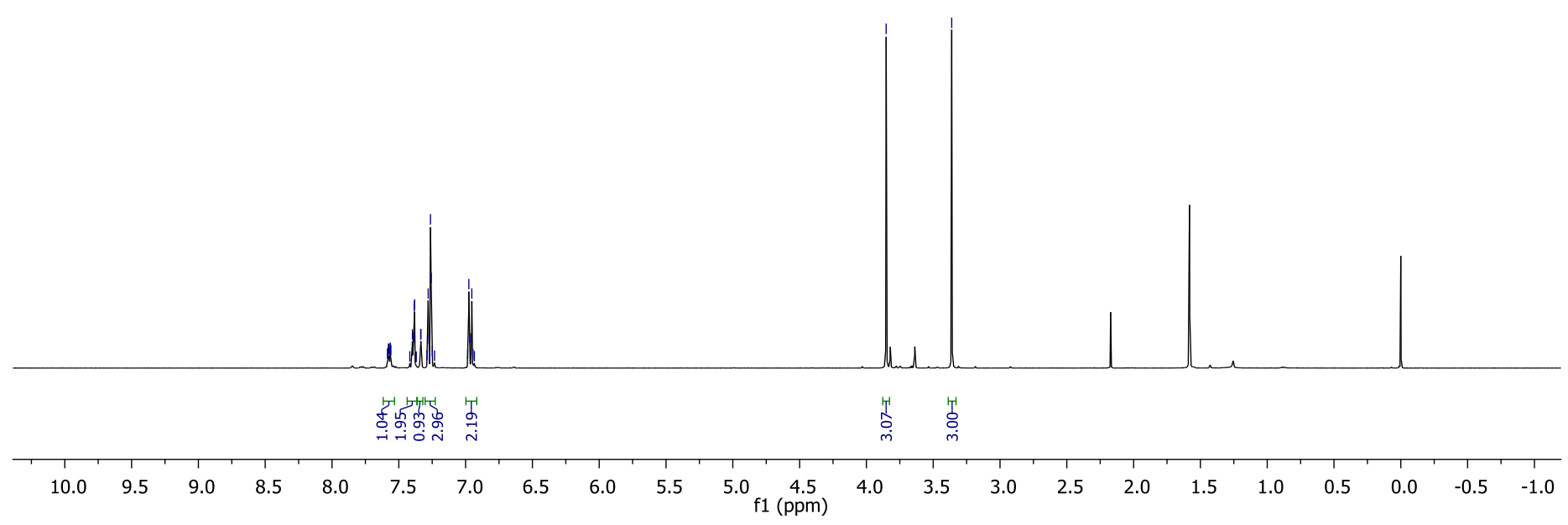




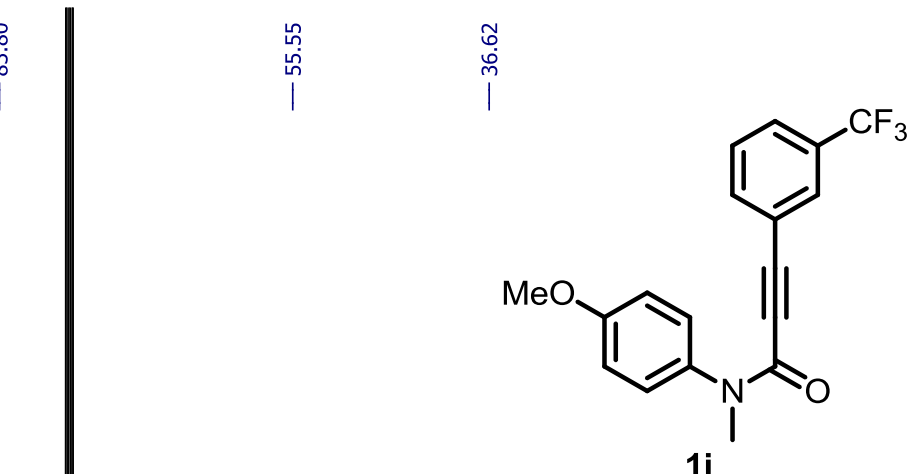

${ }^{13} \mathrm{C} \mathrm{NMR}, \mathrm{CDCl}_{3}, 100 \mathrm{MHz}$
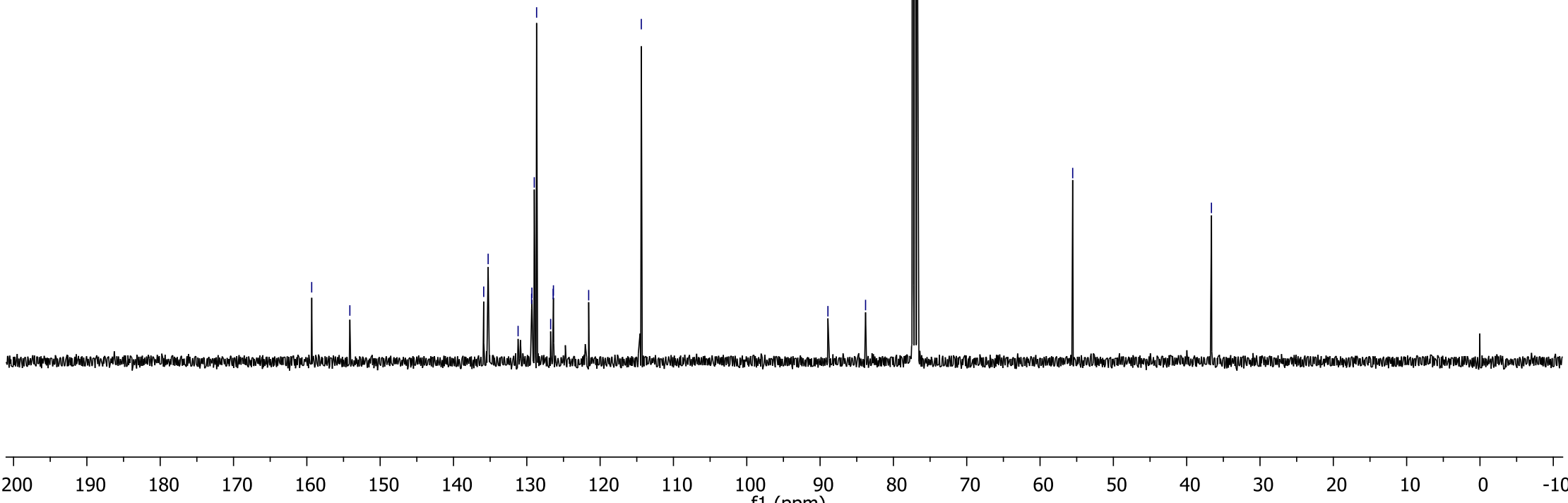


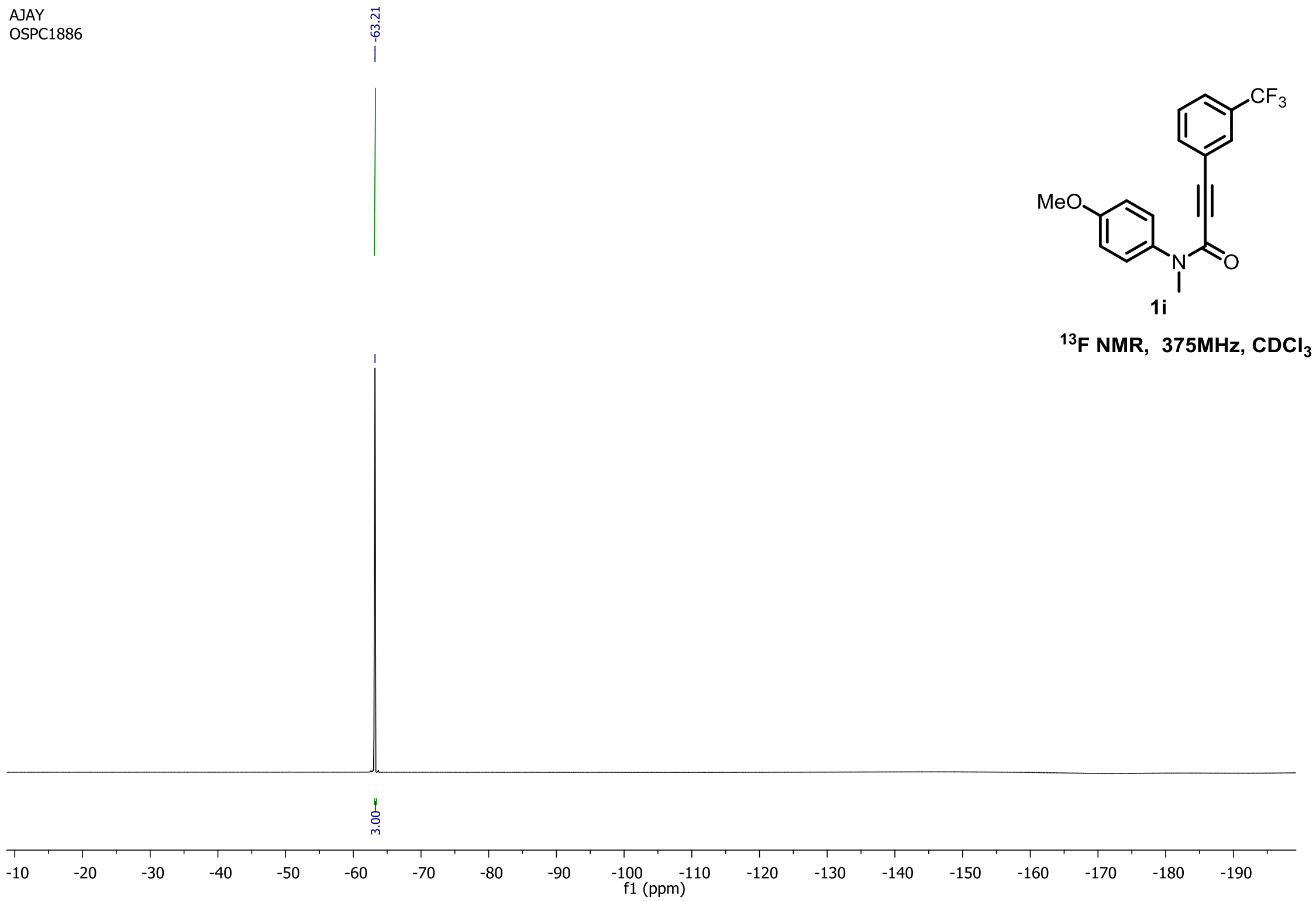



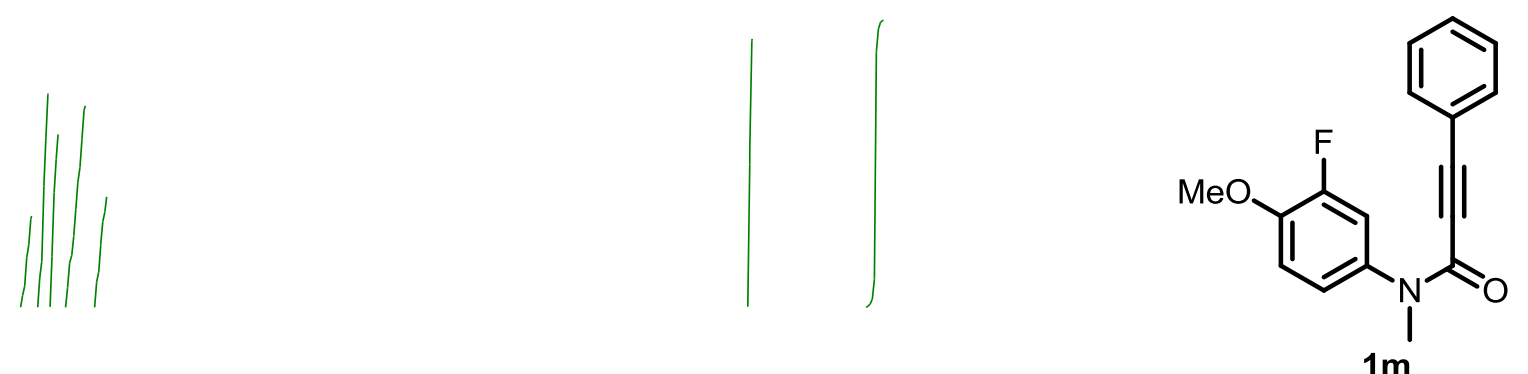

${ }^{1} \mathrm{H}$ NMR, $\mathrm{CDCl}_{3}, 400 \mathrm{MHz}$

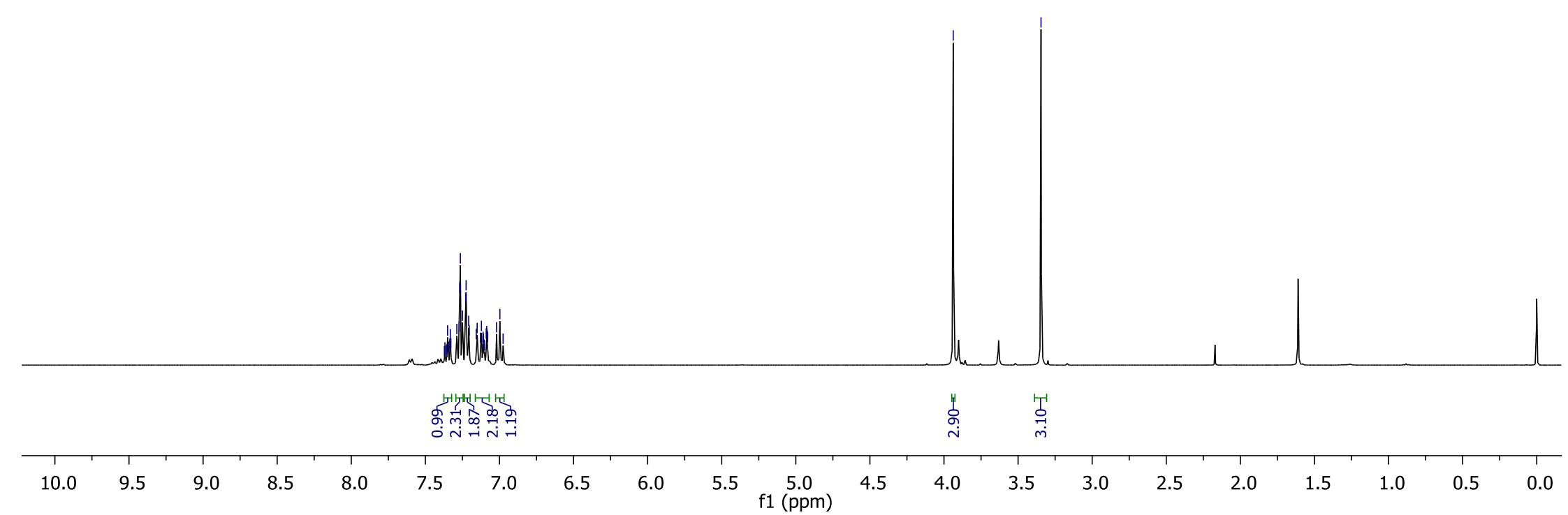



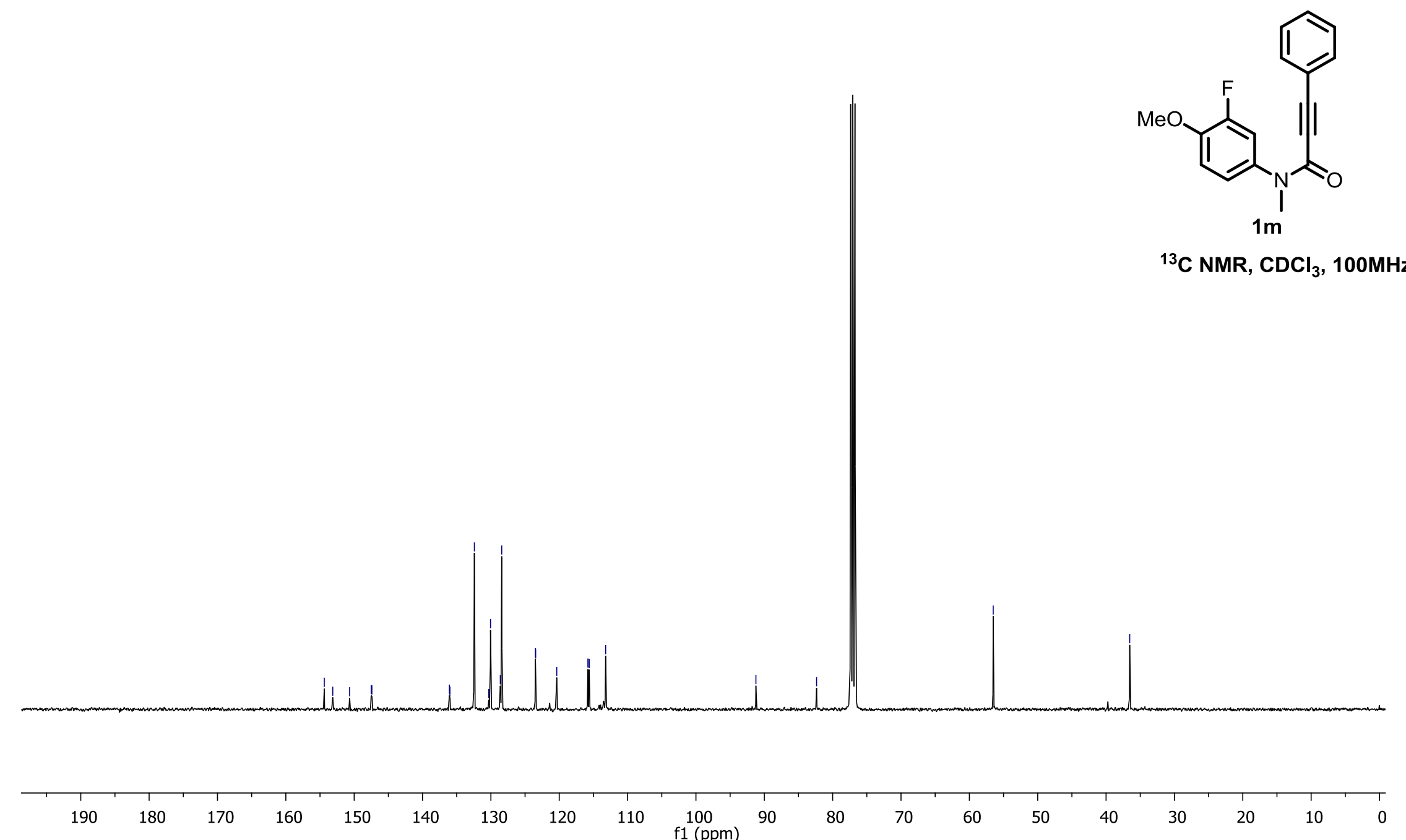
DATTAHAR OSPC1886

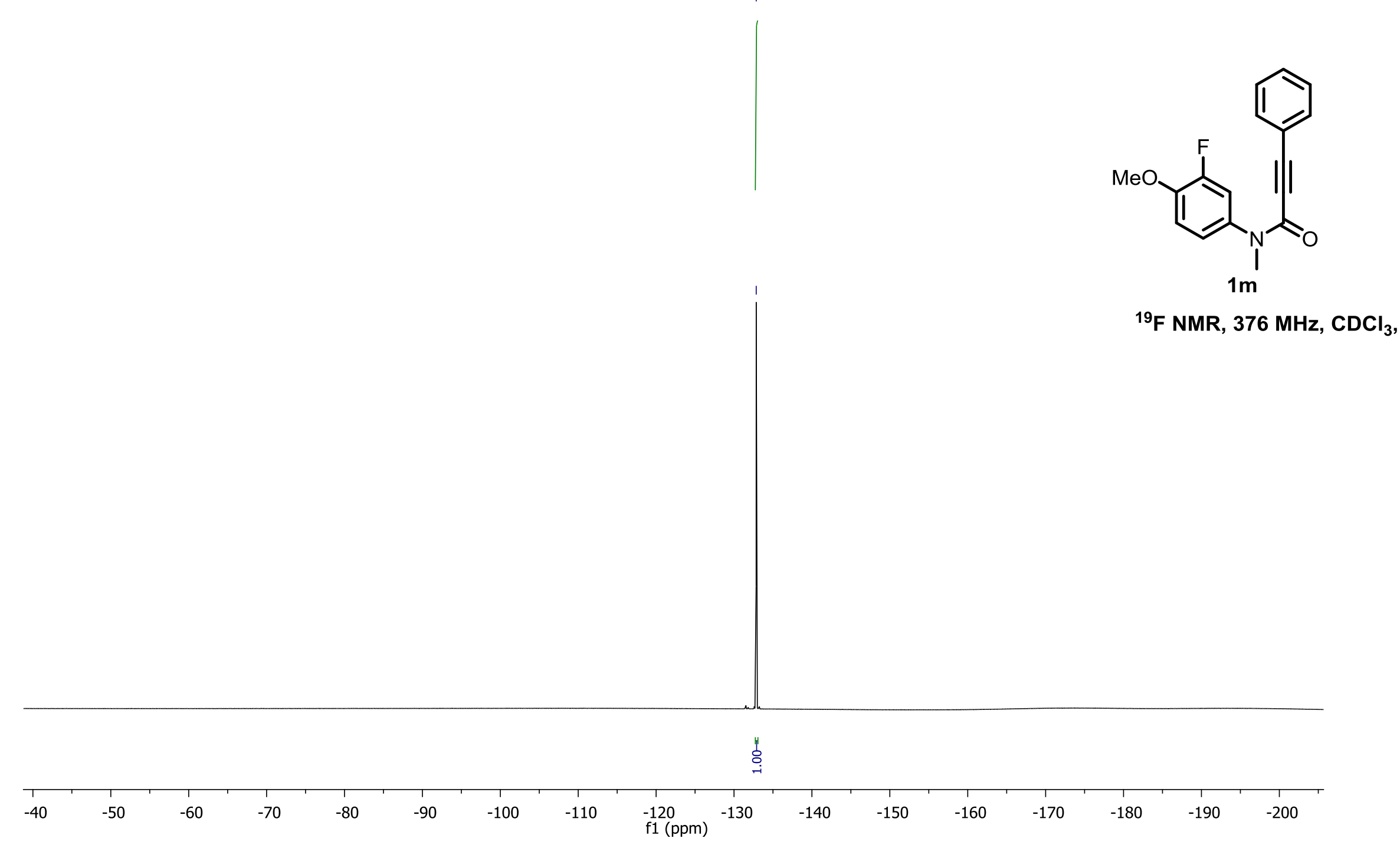




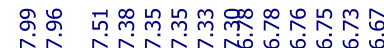

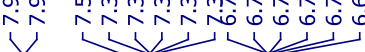

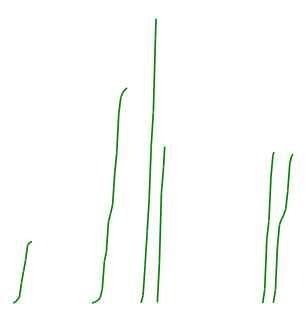

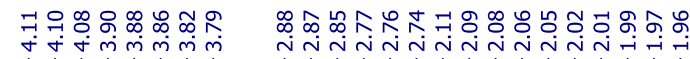

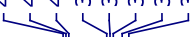
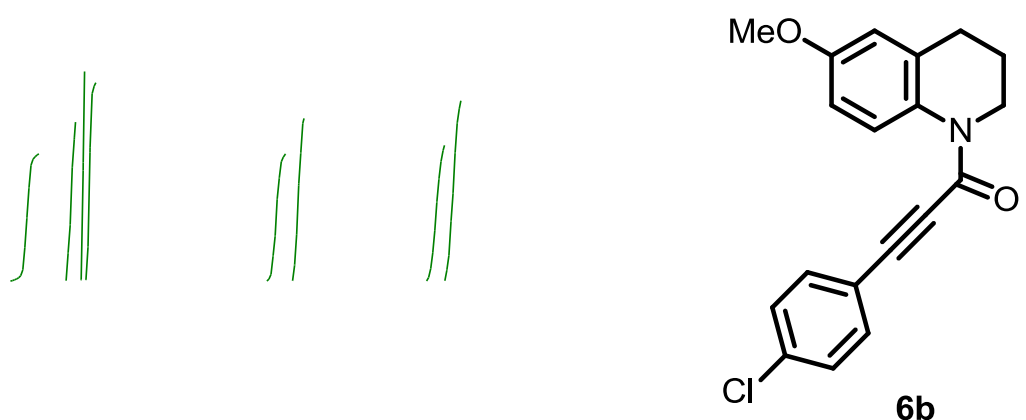

${ }^{1} \mathrm{H}$ NMR, $400 \mathrm{MHz}, \mathrm{CDCl}_{3}$

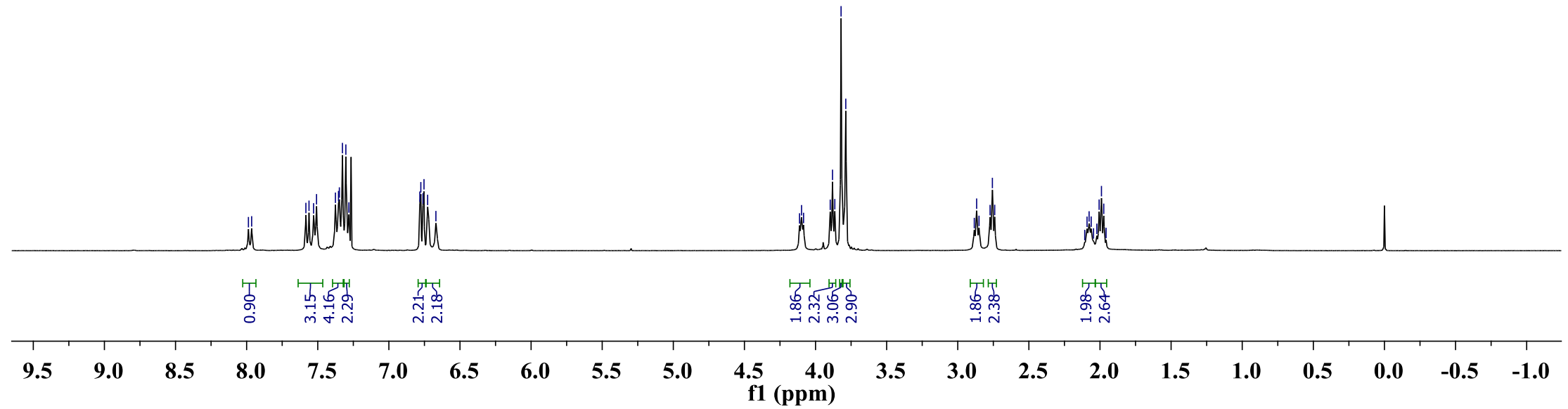




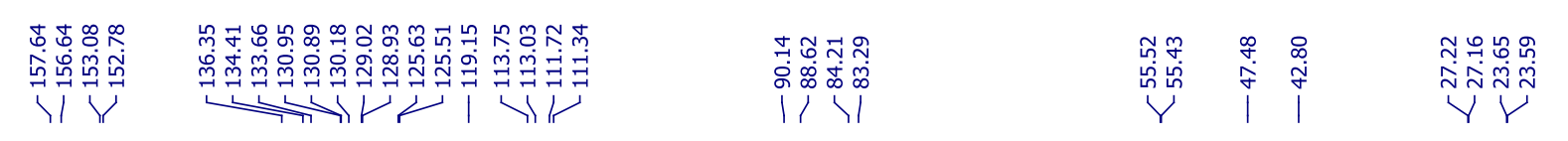

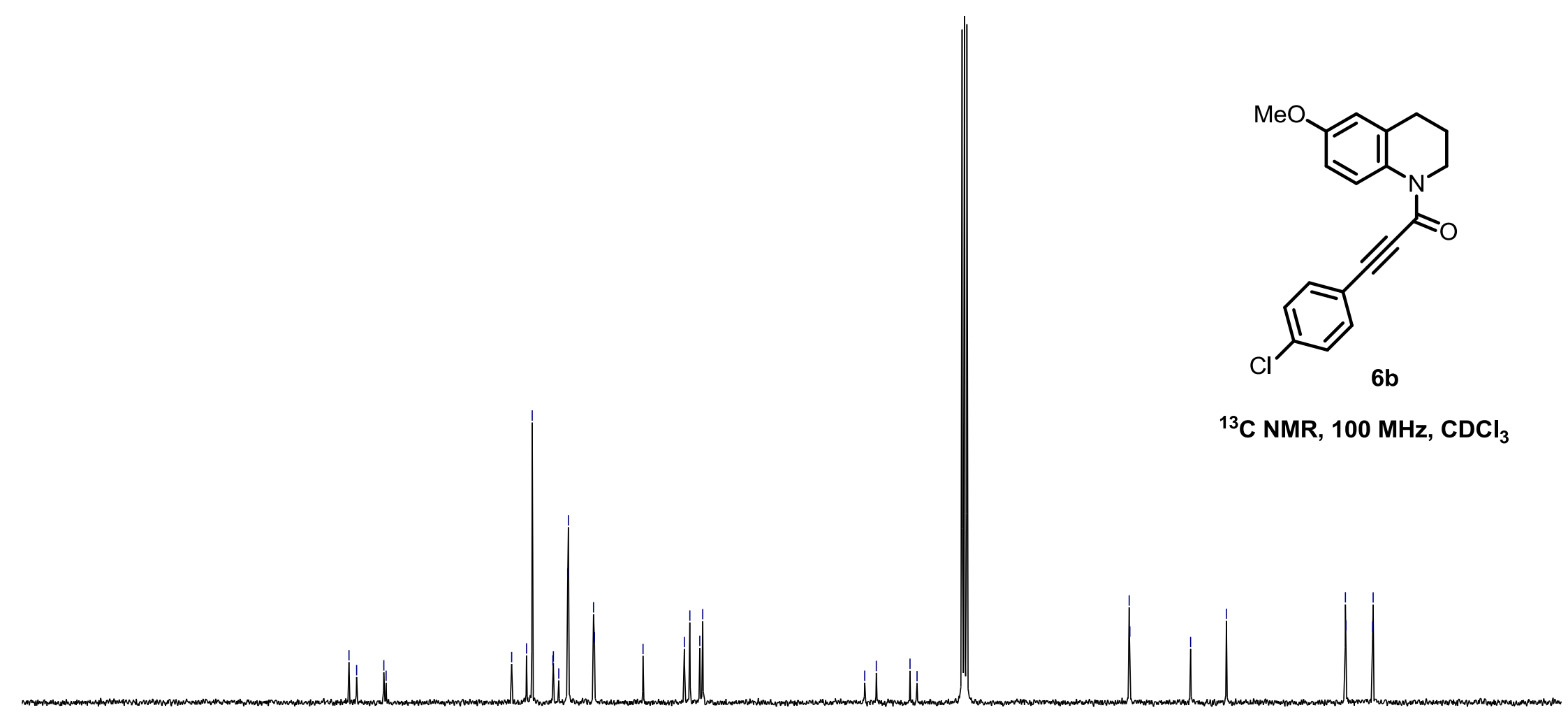




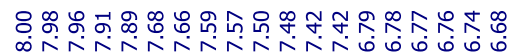

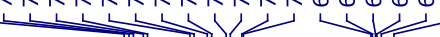

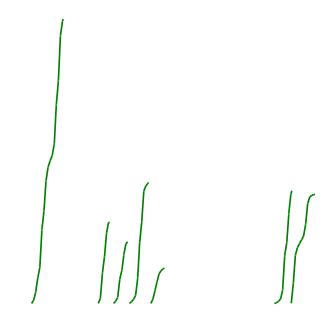

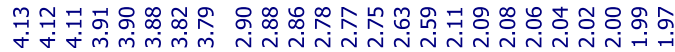

1)

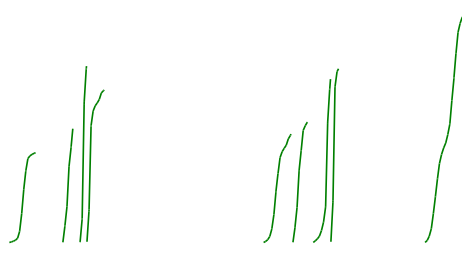

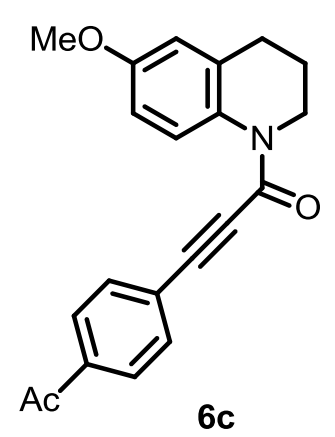

${ }^{1} \mathrm{H}$ NMR, $400 \mathrm{MHz}, \mathrm{CDCl}_{3}$

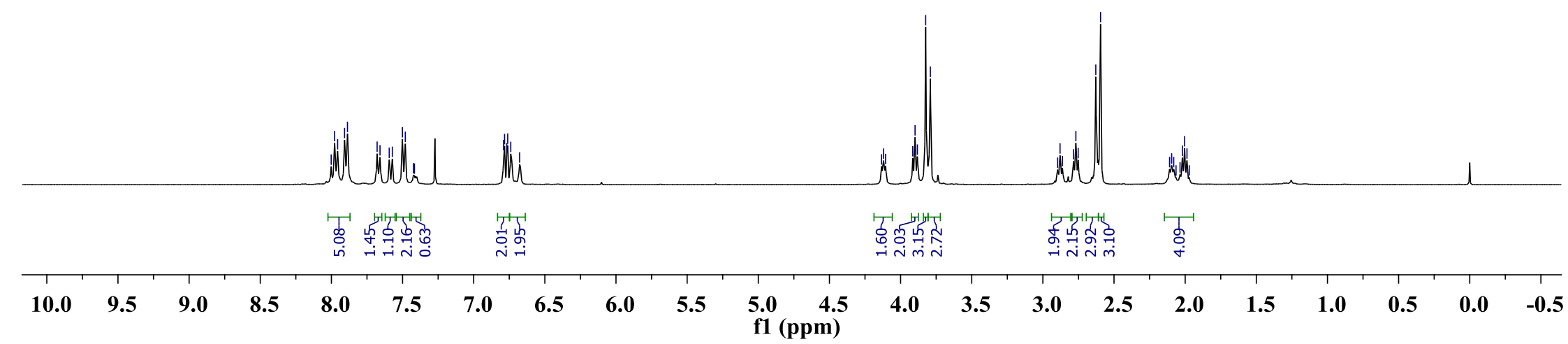



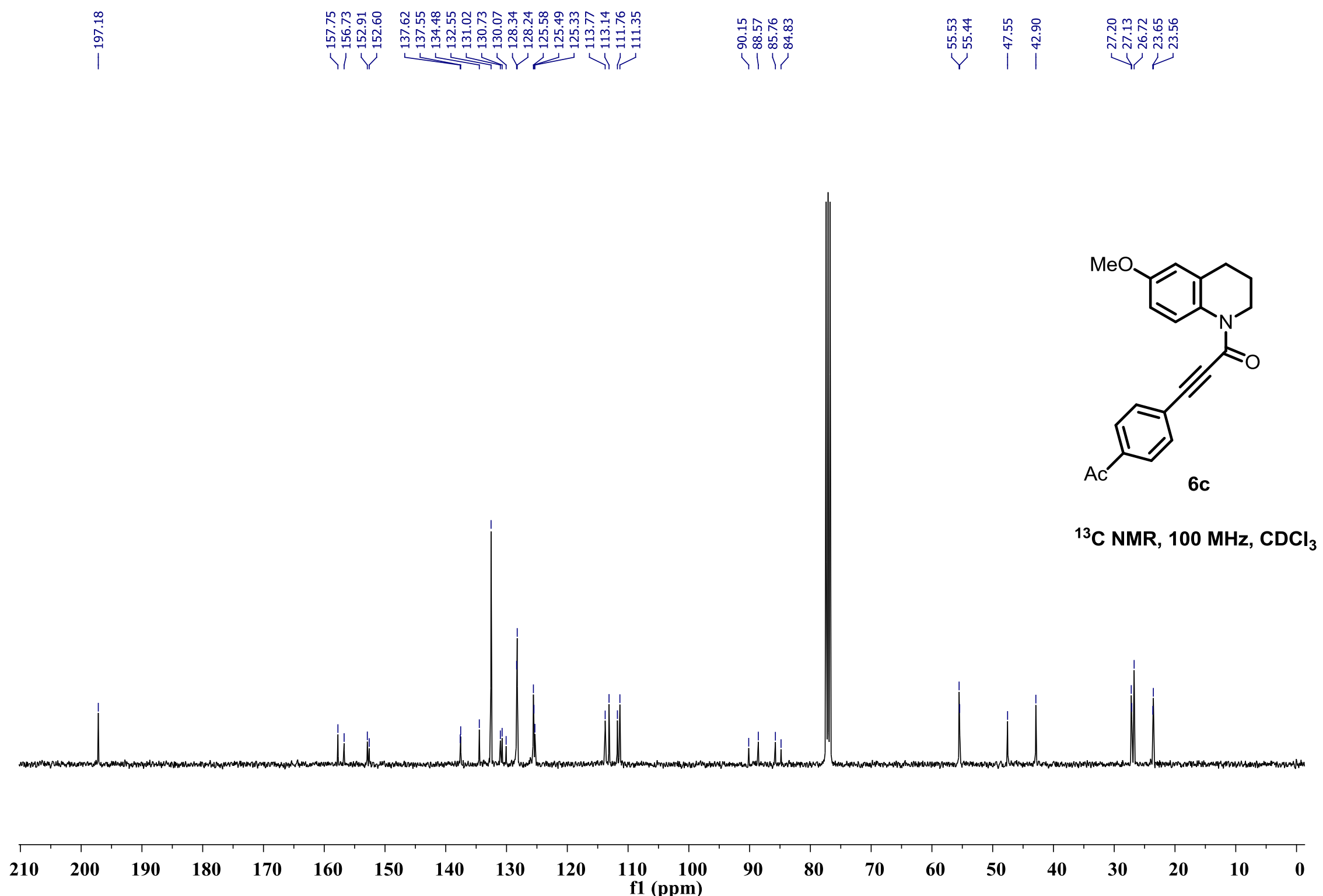


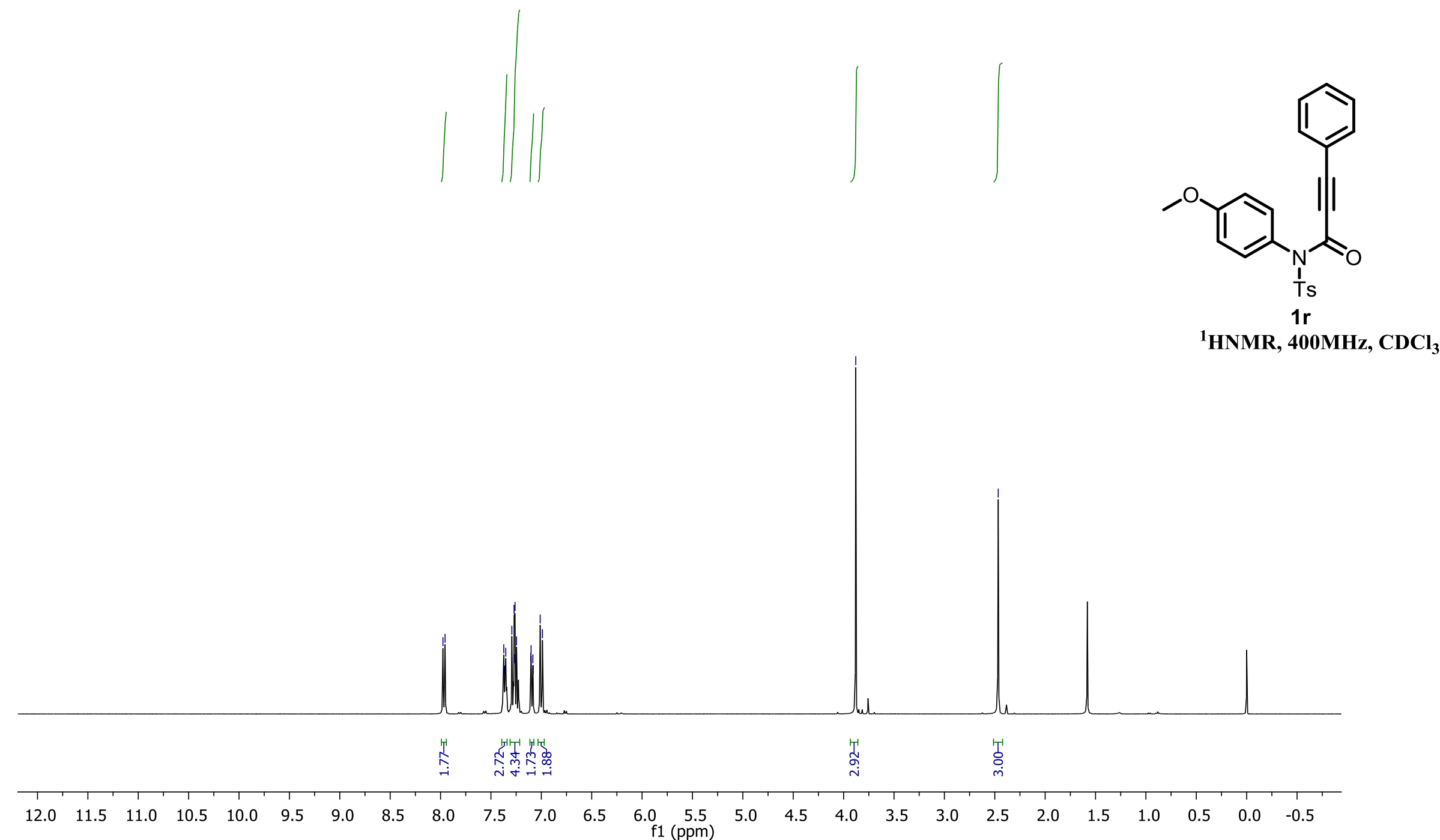




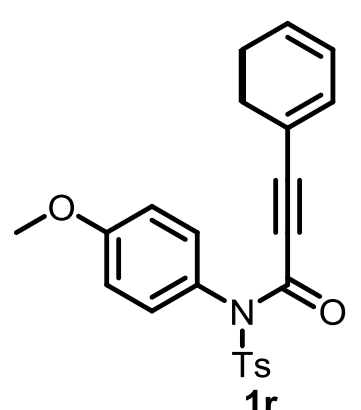

${ }^{13} \mathrm{CNMR}, 100 \mathrm{MHz}, \mathrm{CDCl}_{3}$,
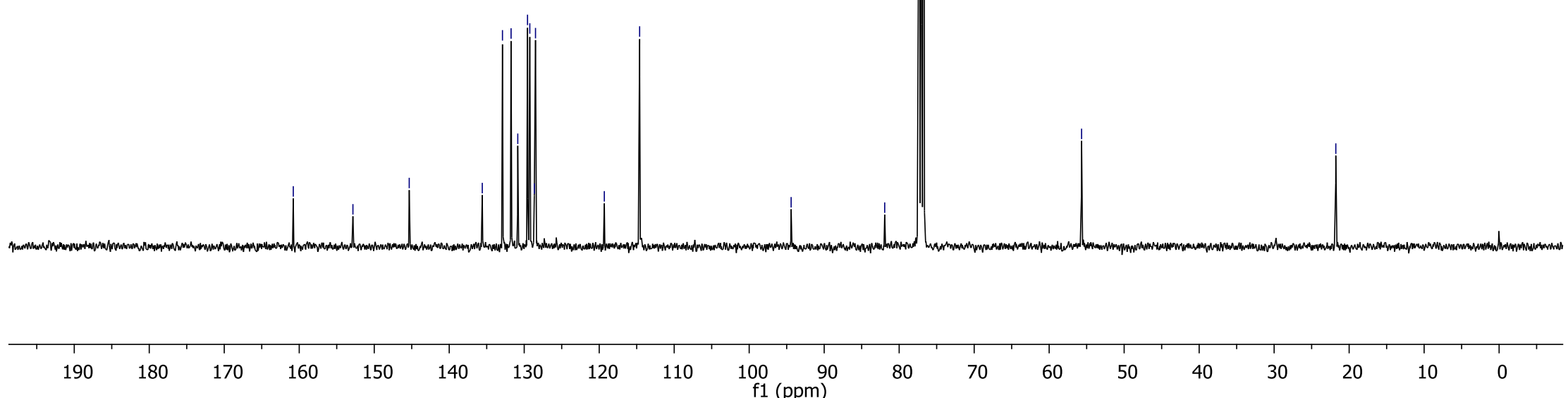


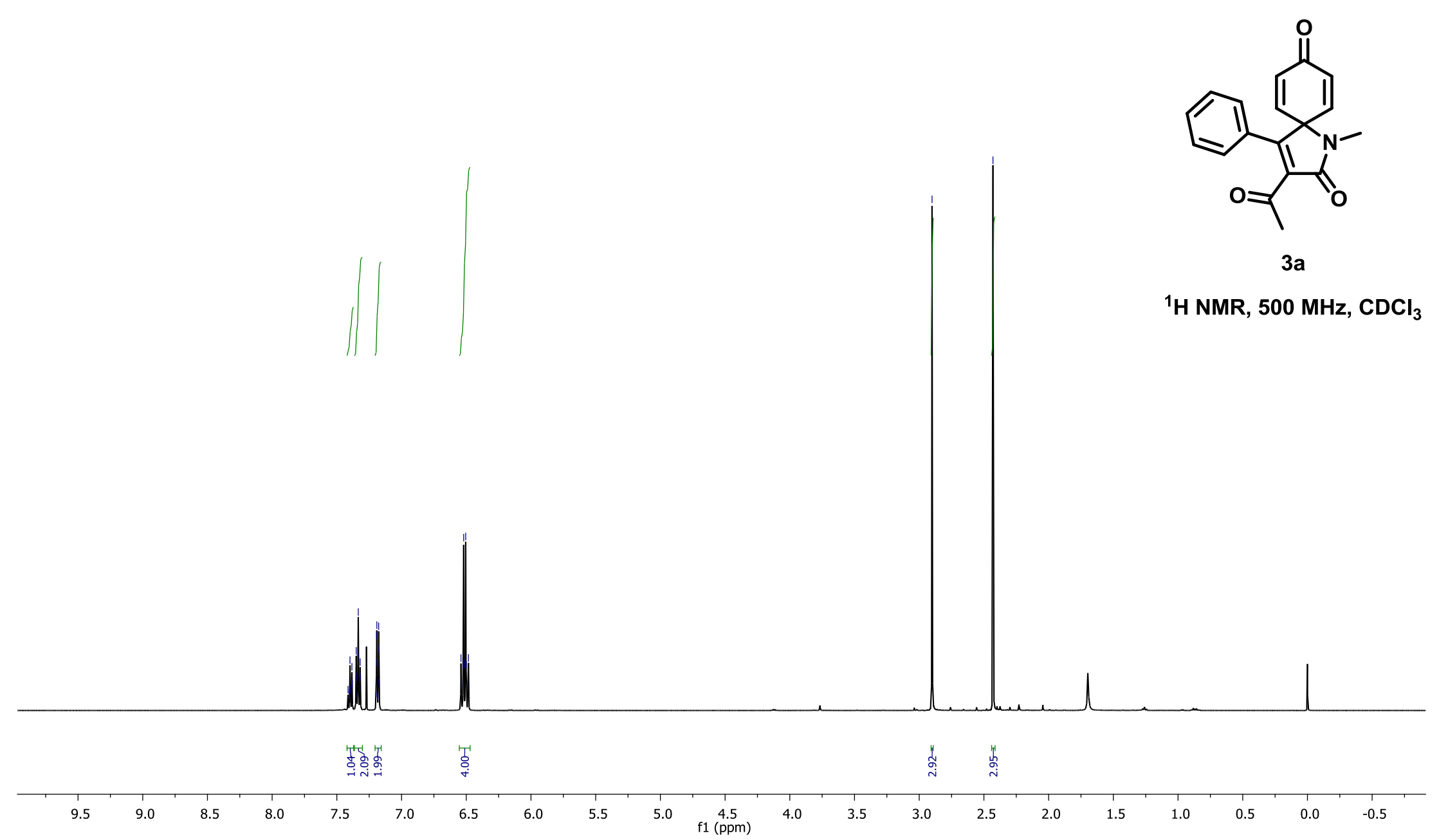




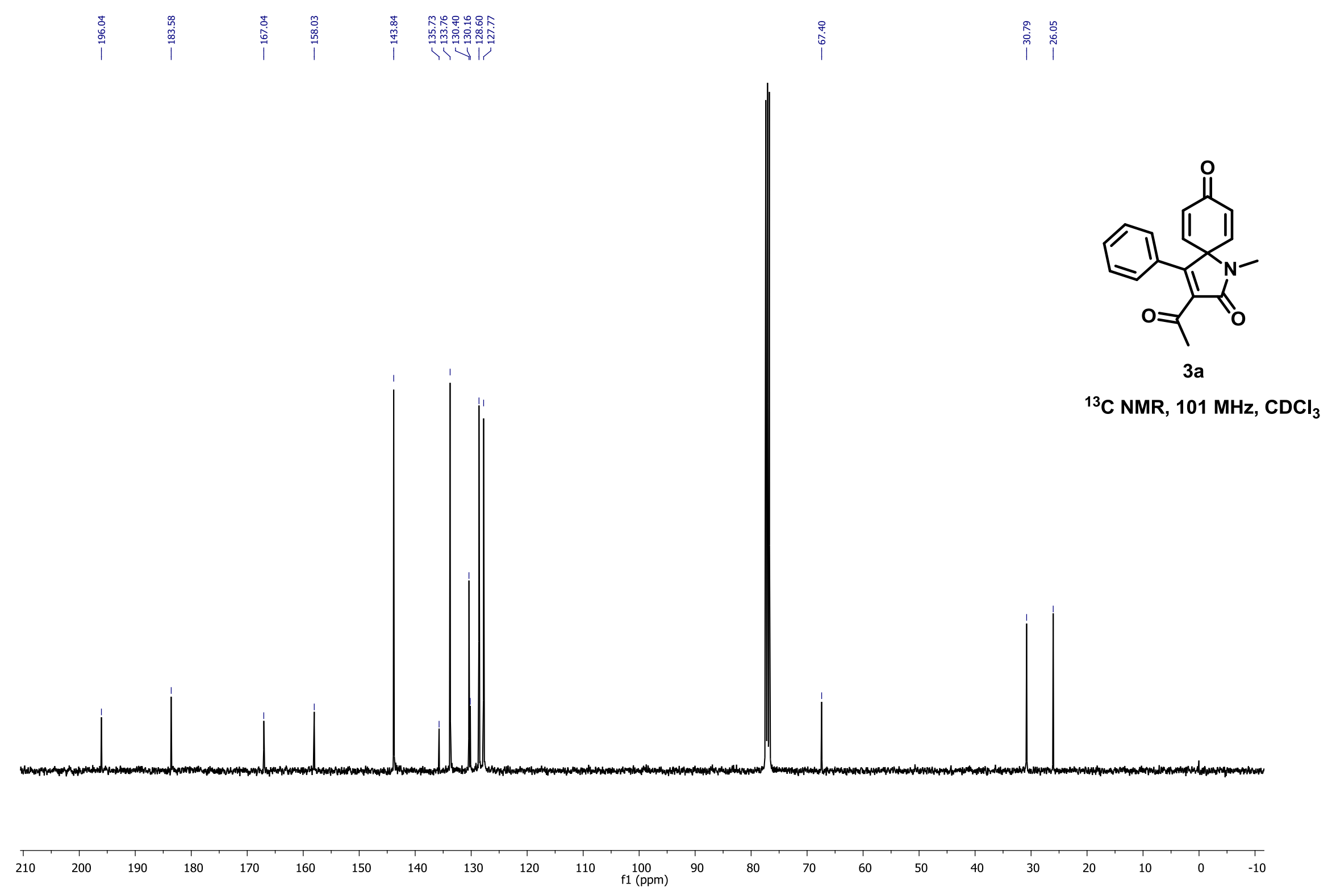




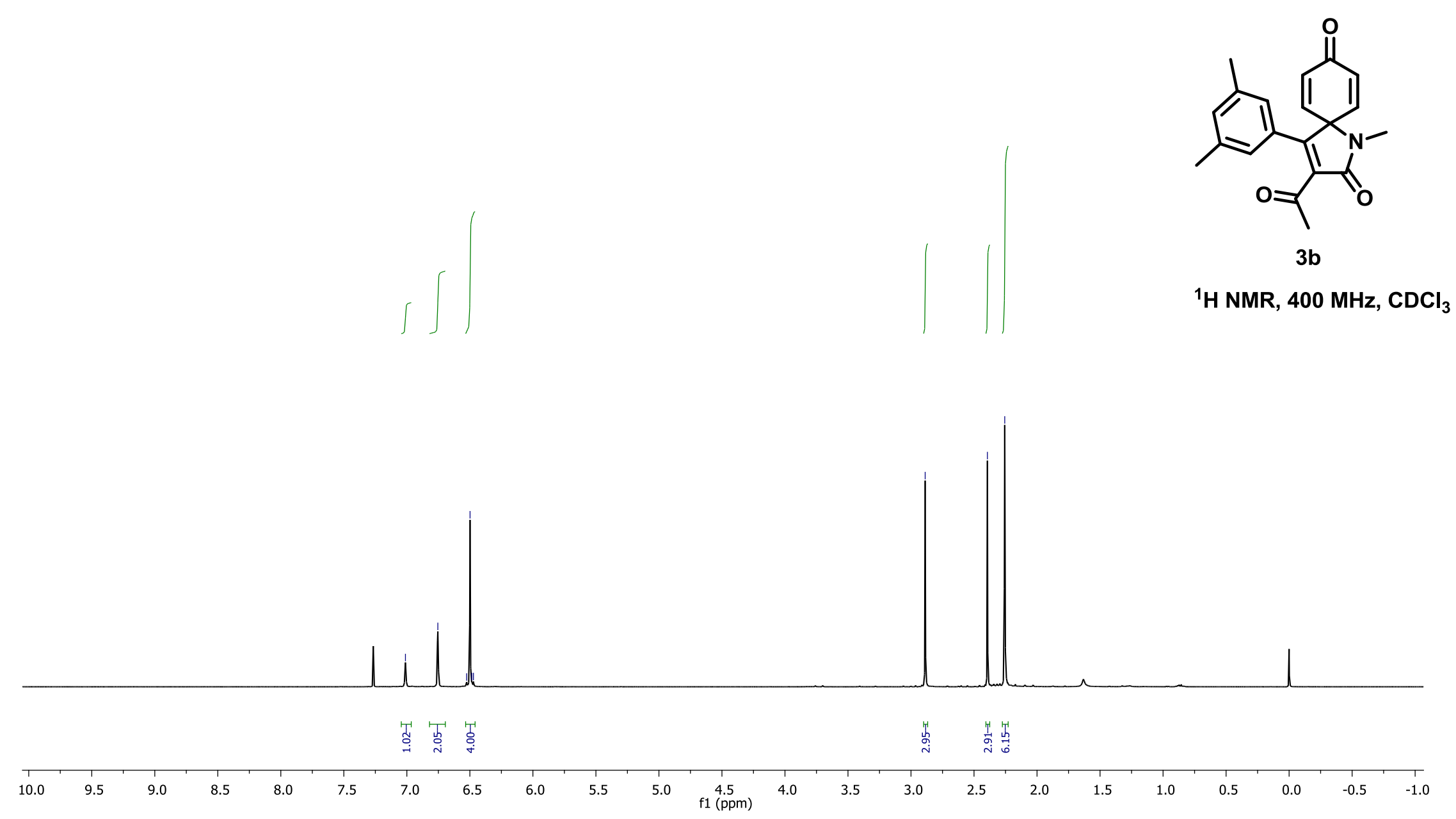



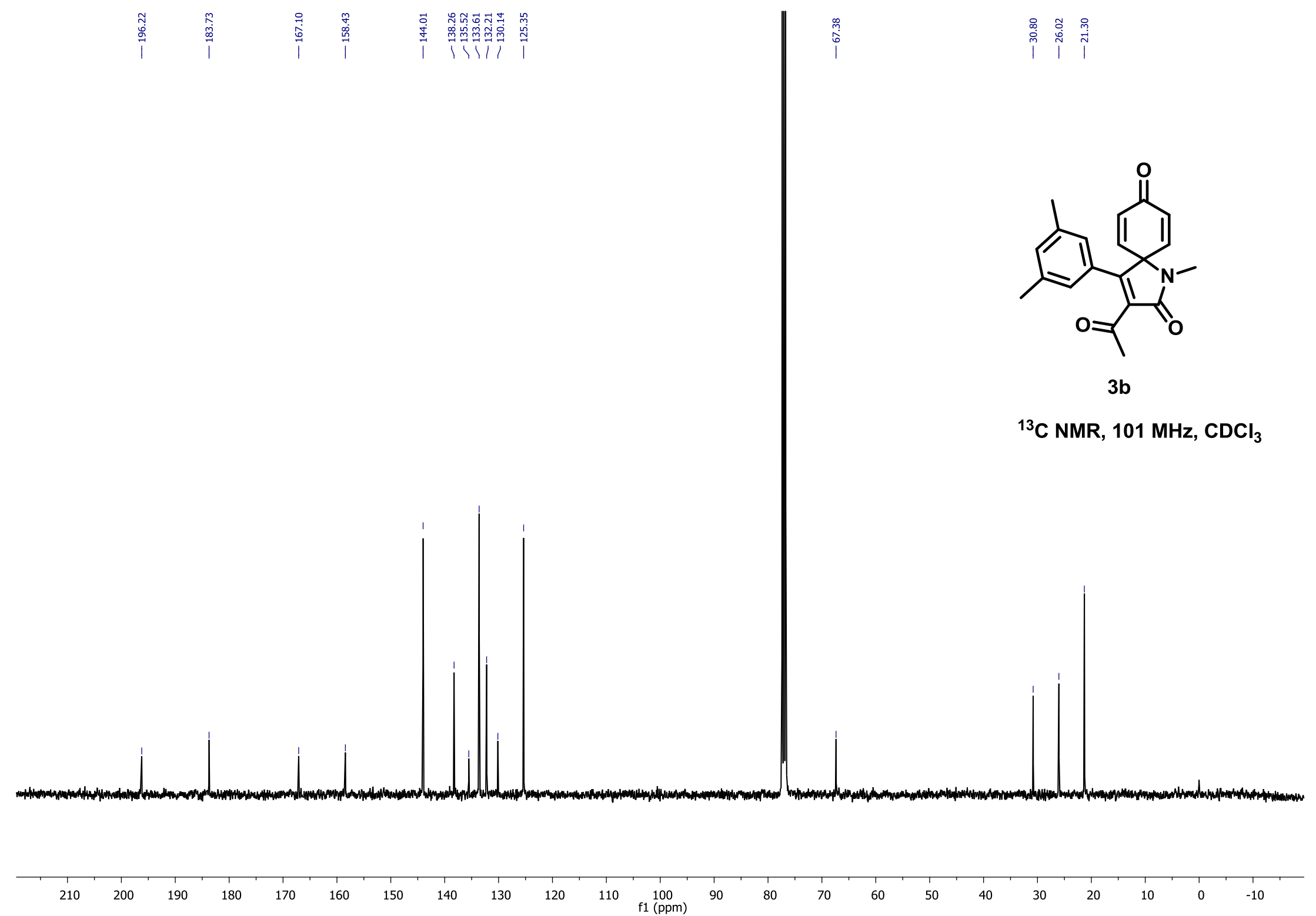


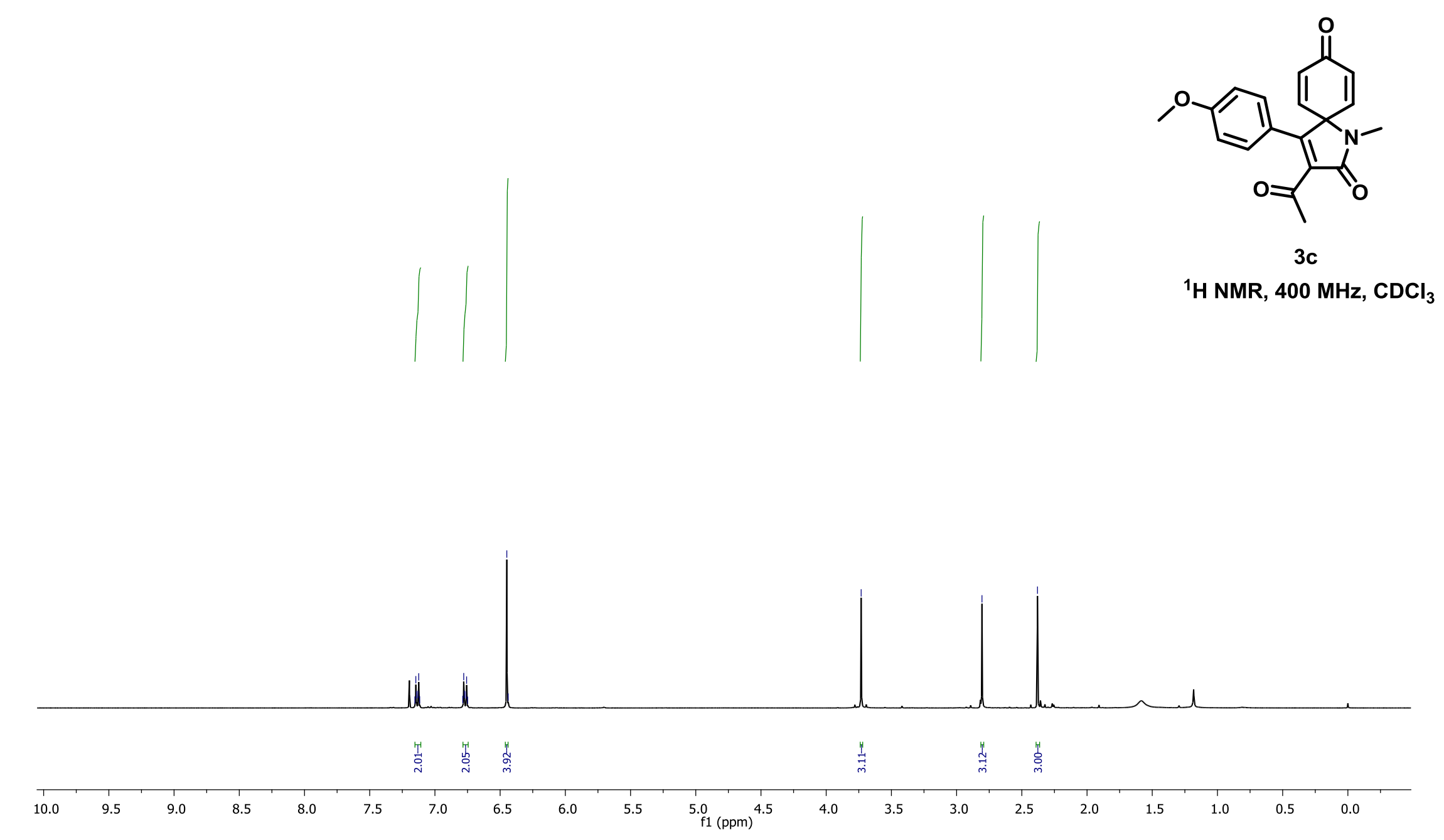




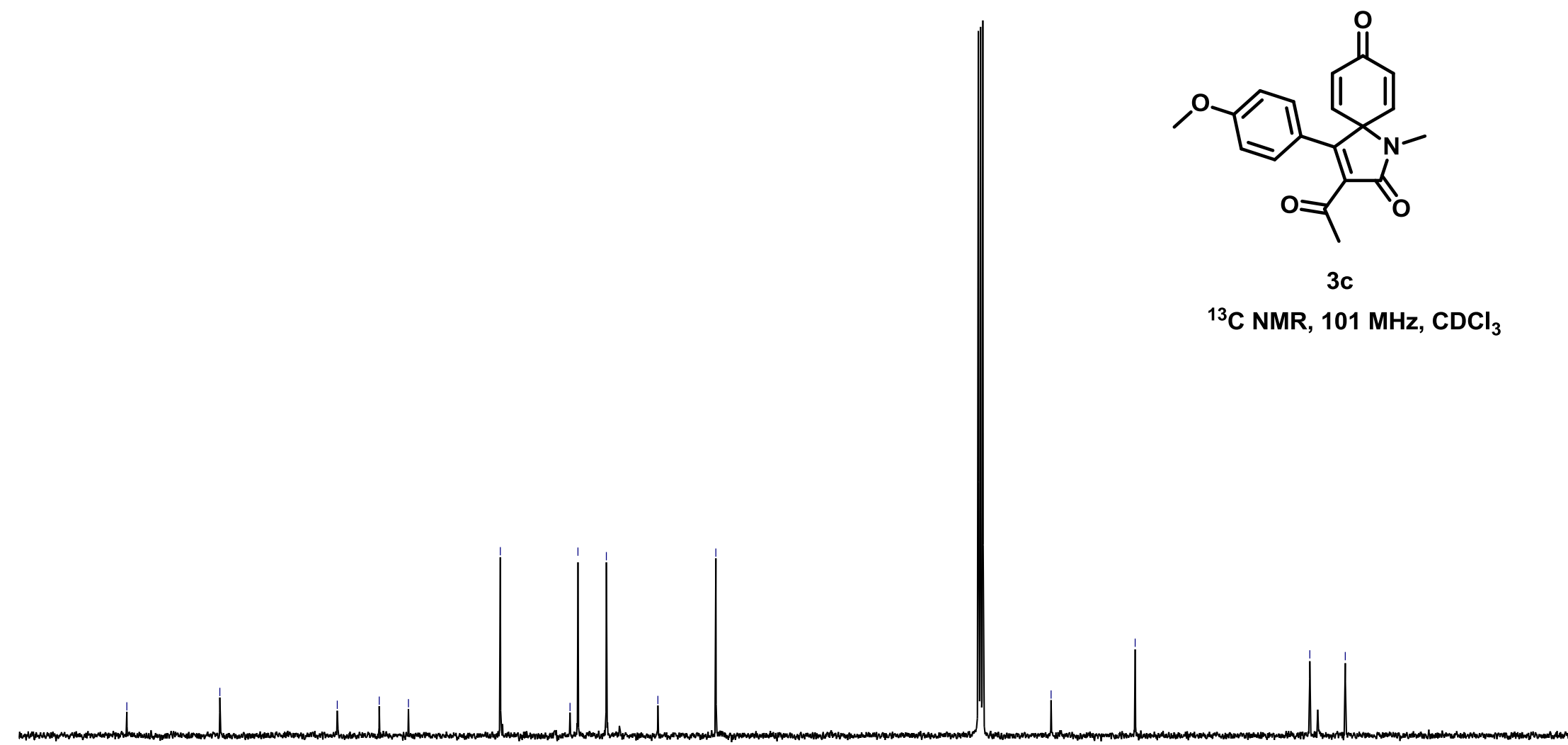




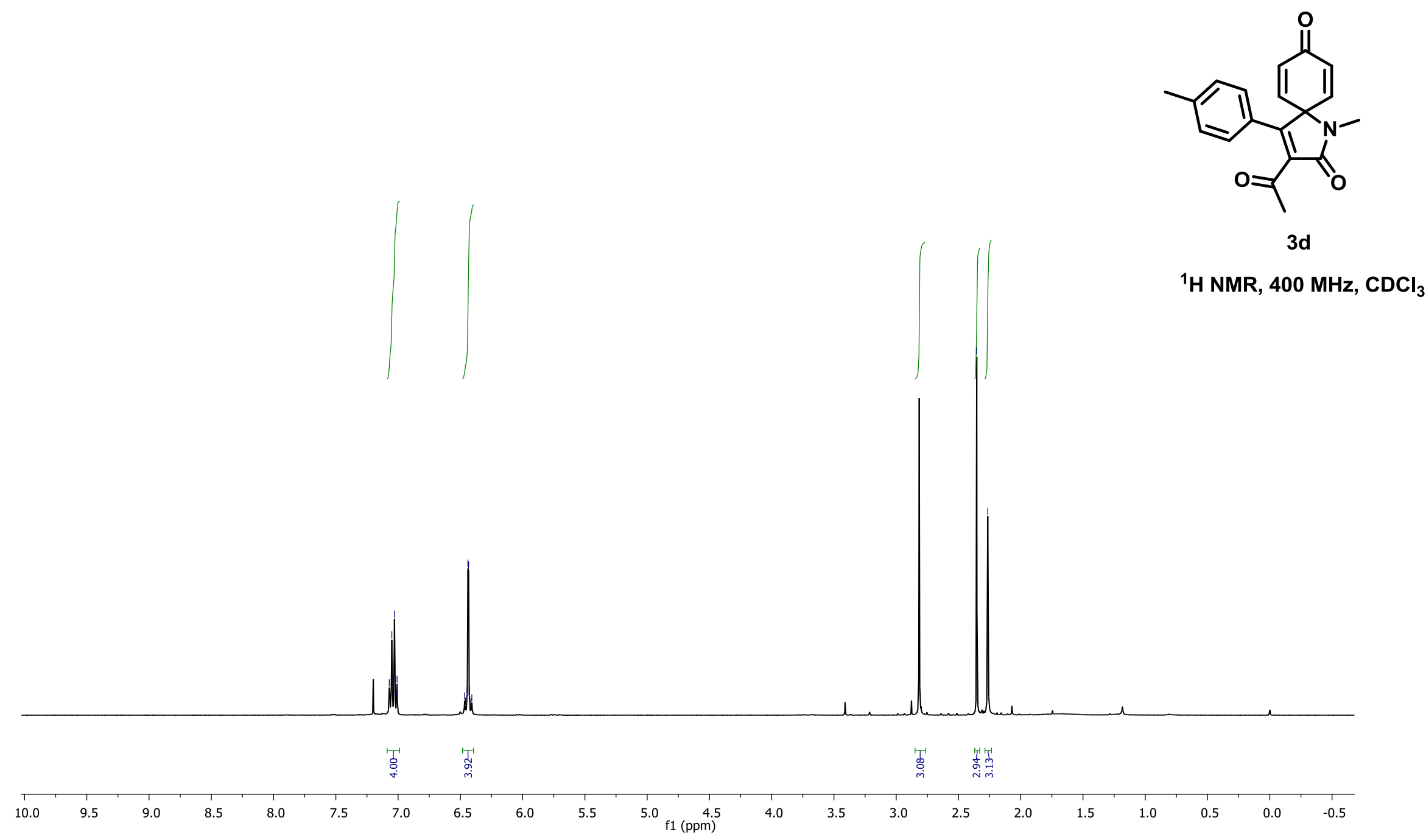



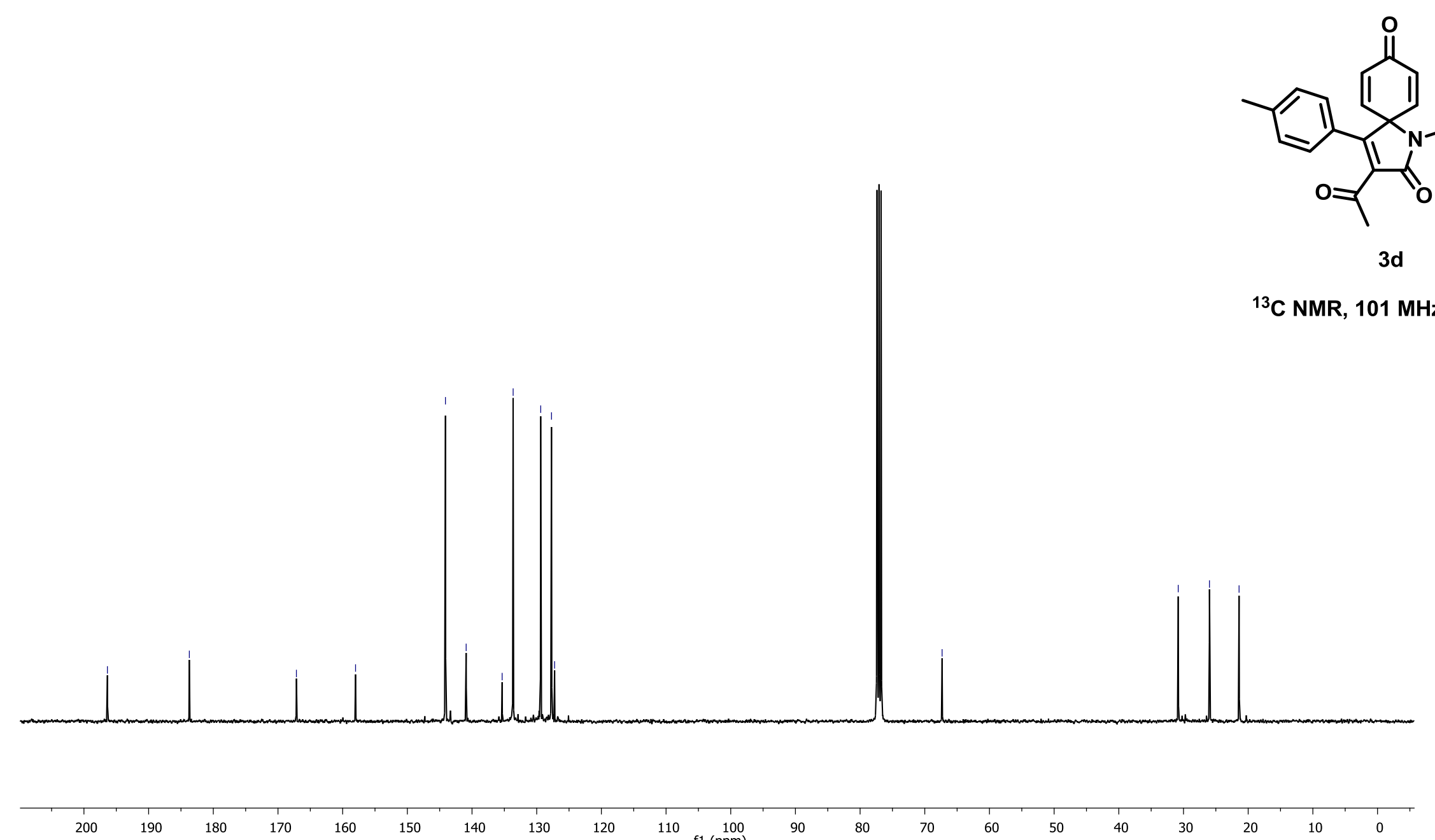


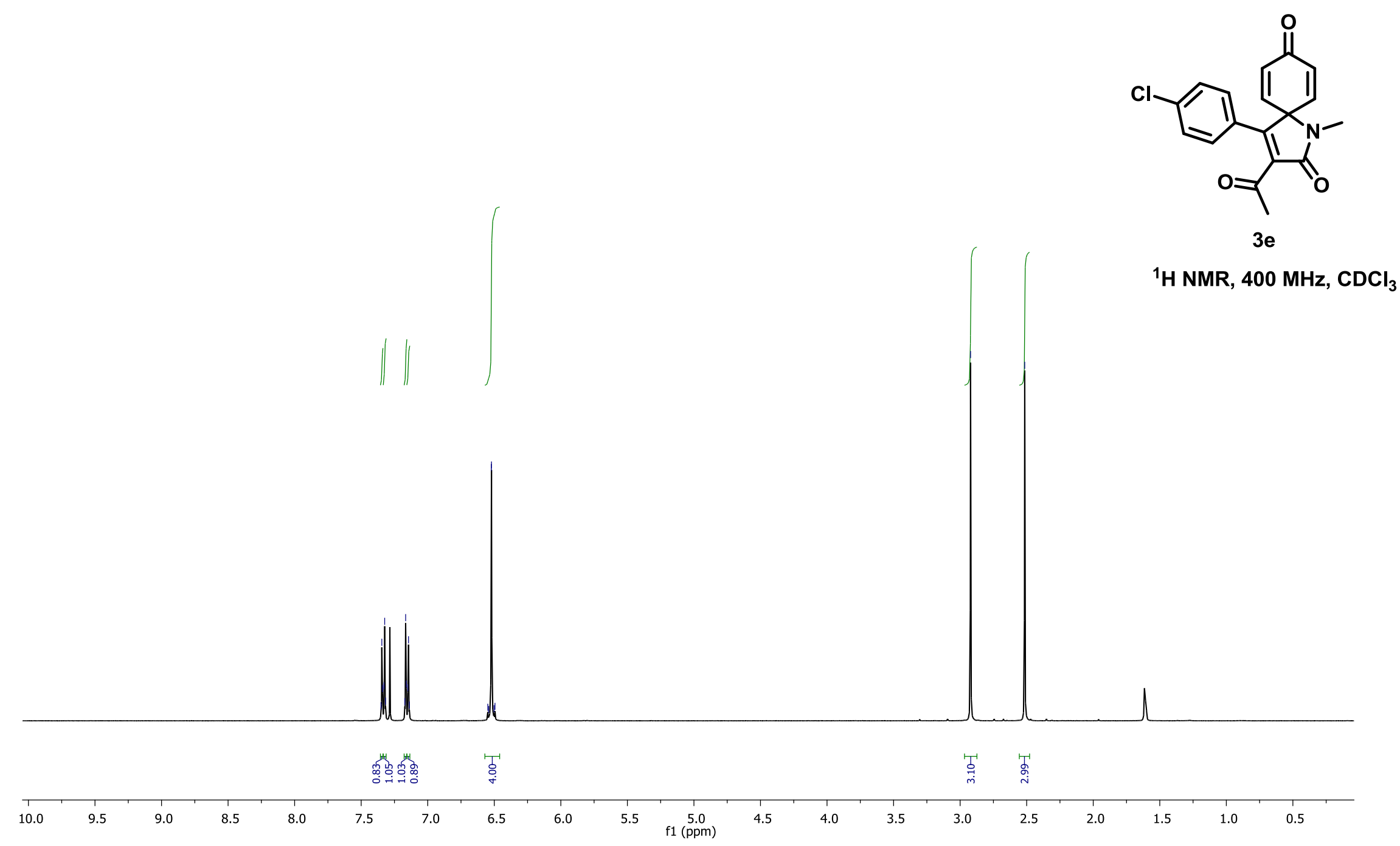



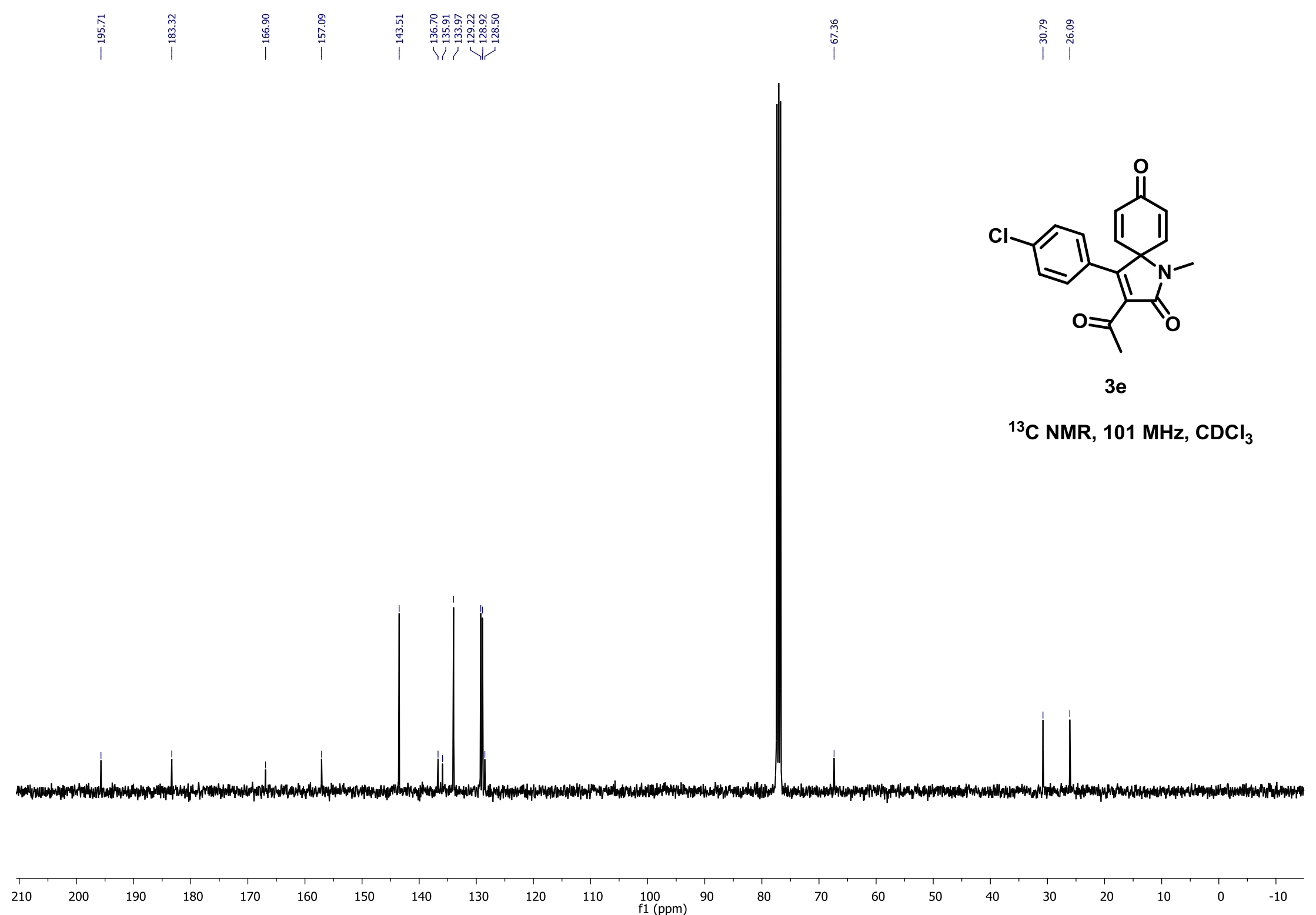


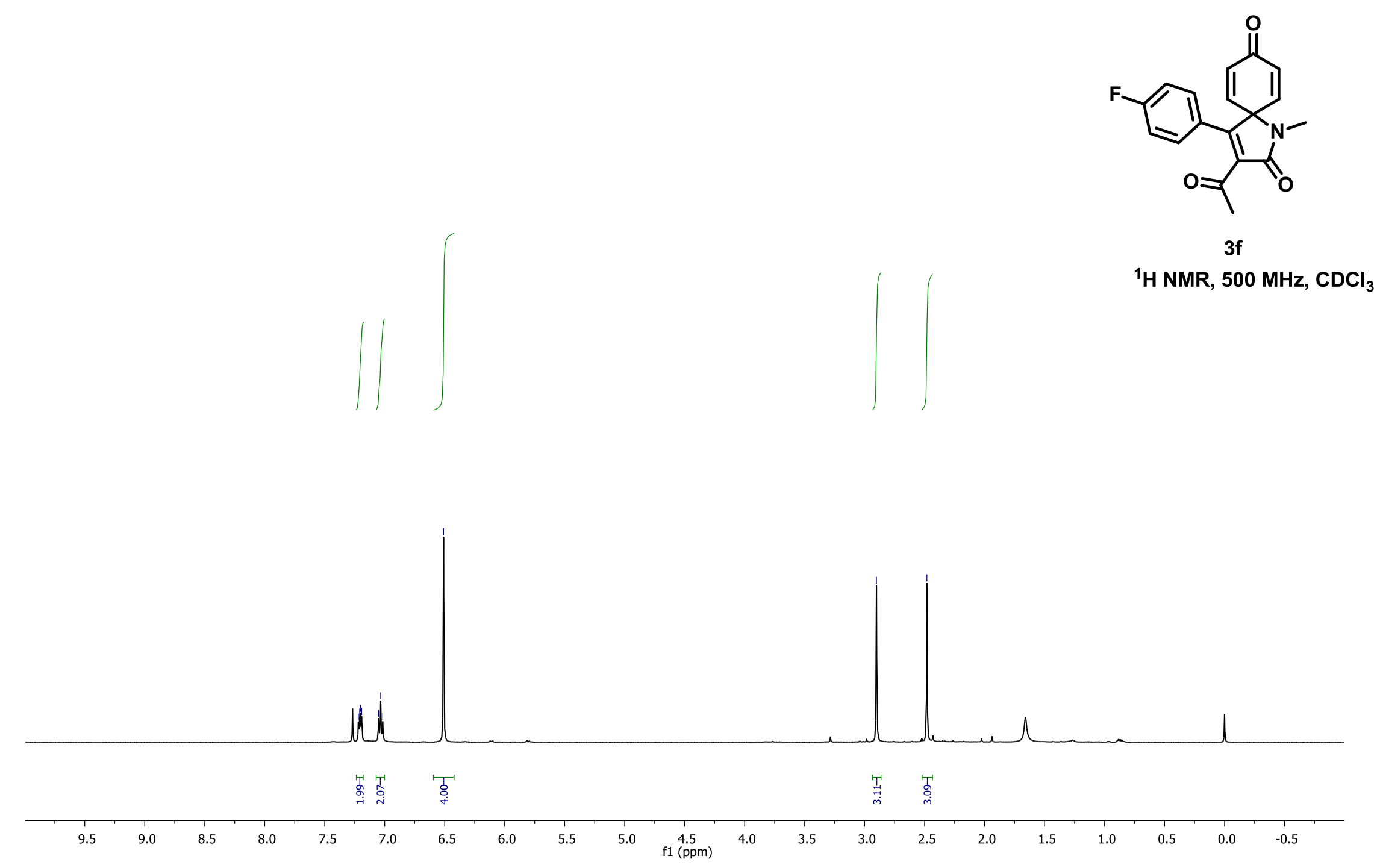




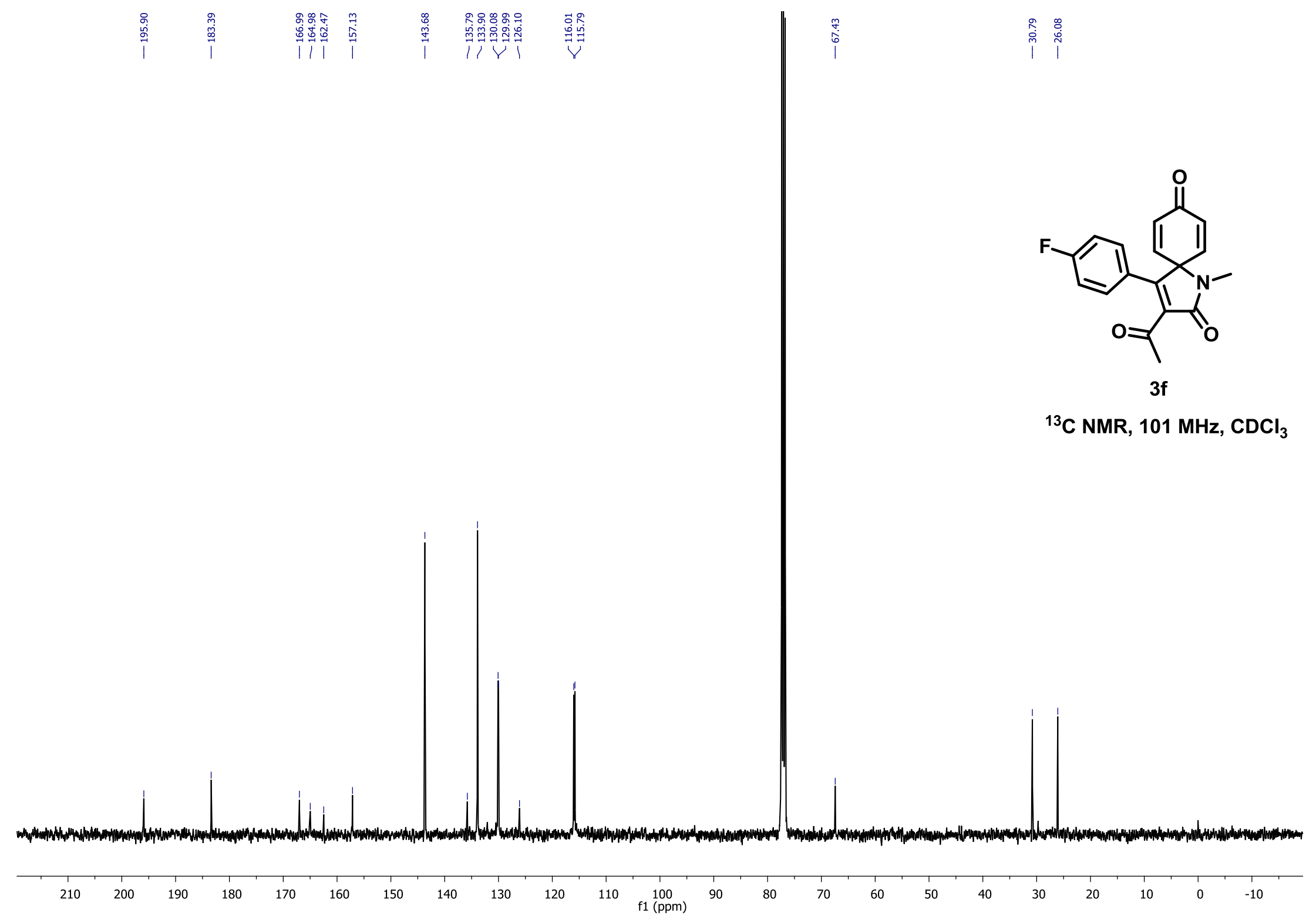


M SUBBA RAO

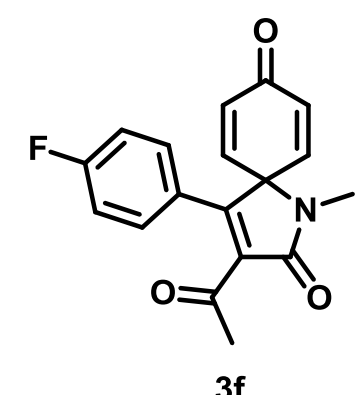

${ }^{19} \mathrm{~F}$ NMR, $376 \mathrm{MHz}^{\mathrm{CDCl}}$

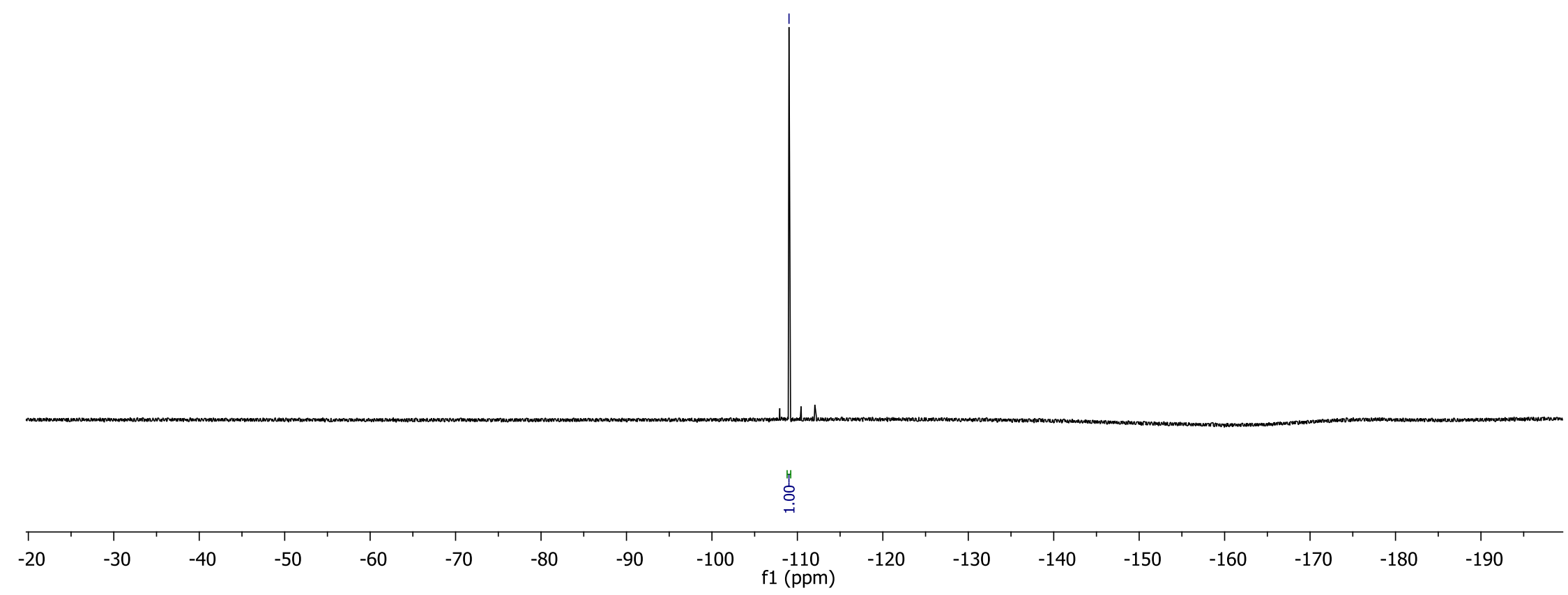




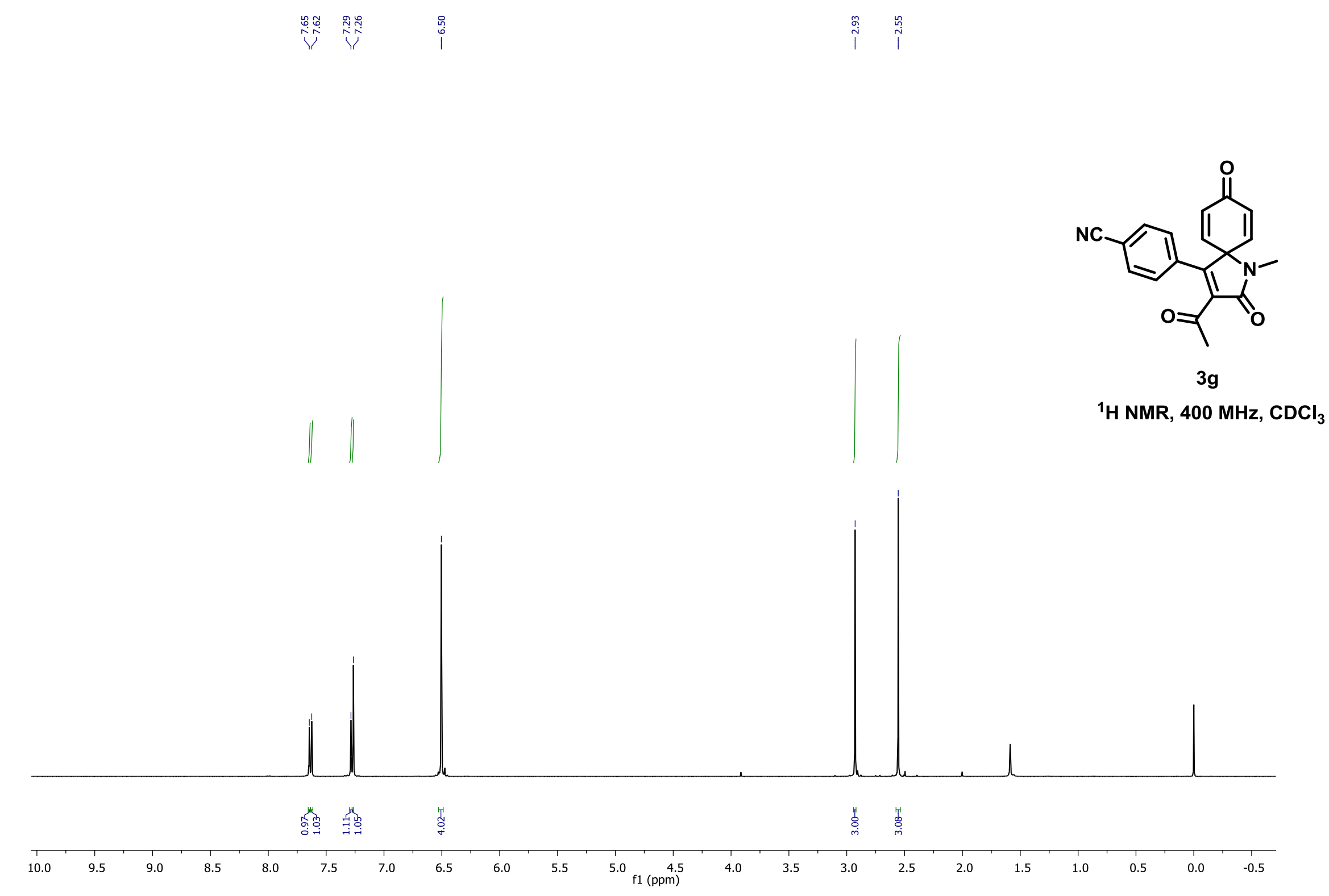



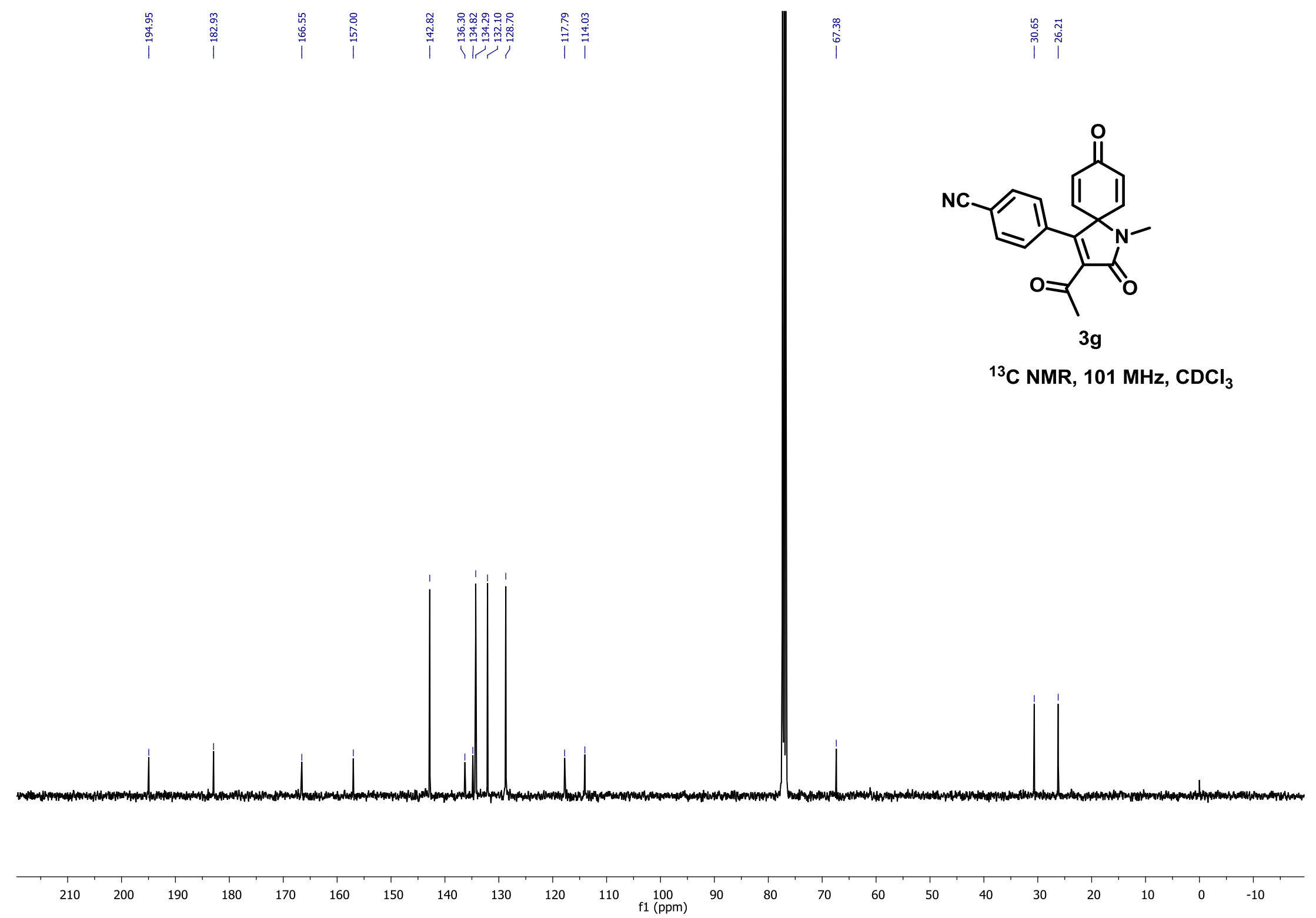


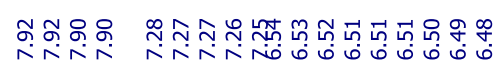

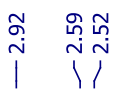
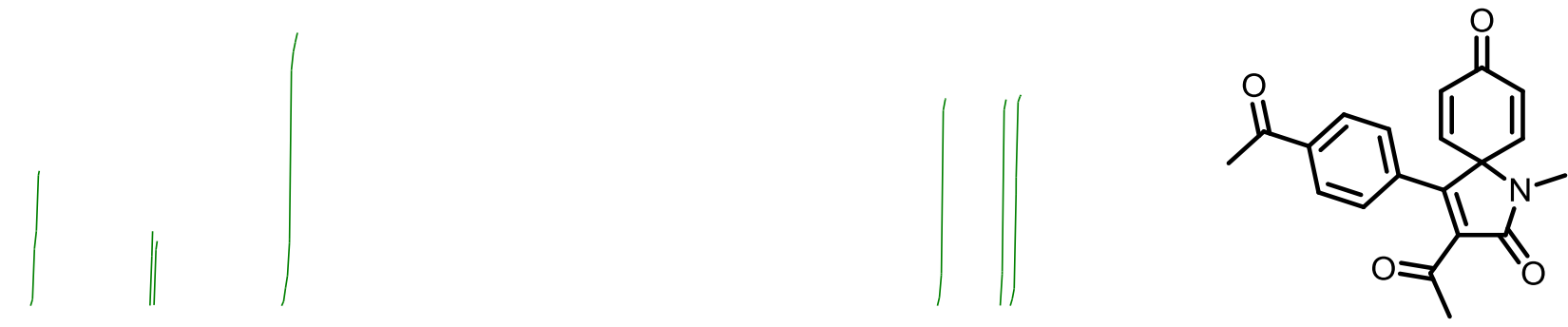

3h

${ }^{1} \mathrm{H}$ NMR, $400 \mathrm{MHz}, \mathrm{CDCl}_{3}$

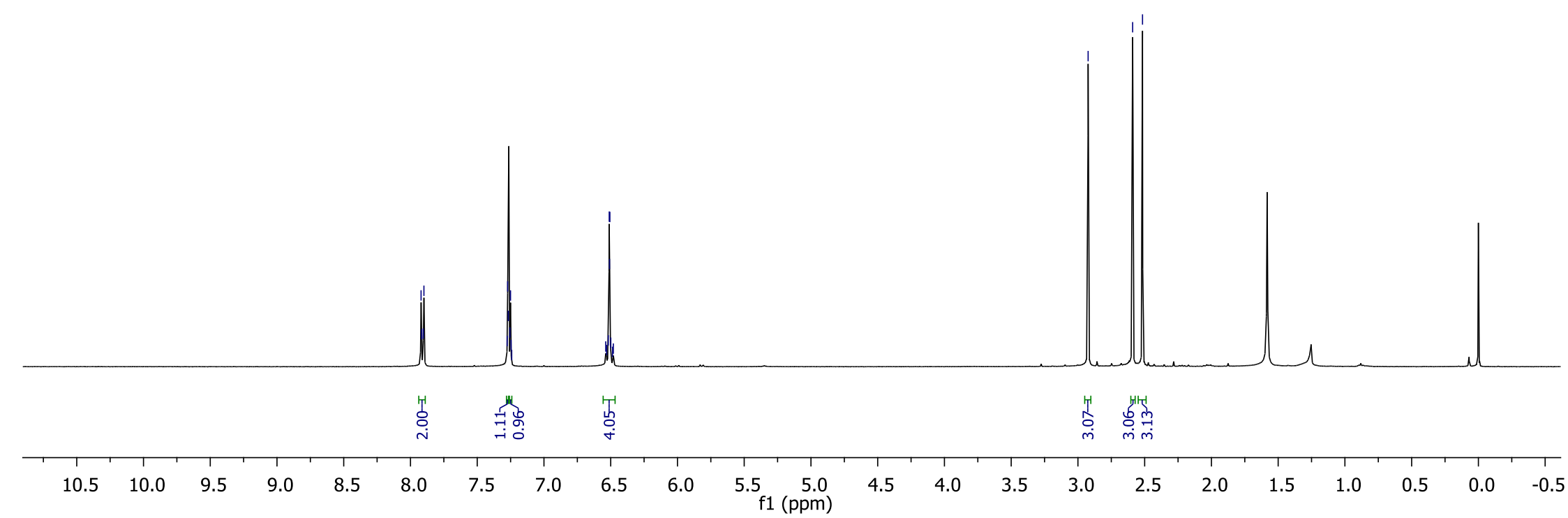




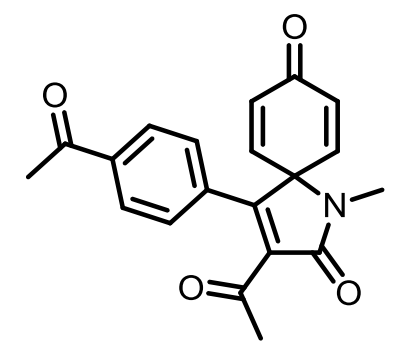

3h

${ }^{13} \mathrm{C}$ NMR, $101 \mathrm{MHz}, \mathrm{CDCl}_{3}$

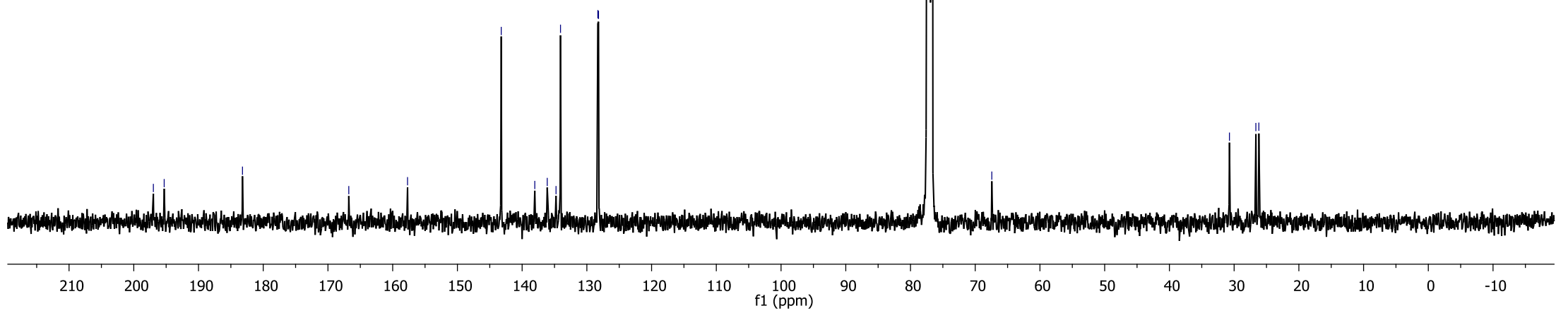




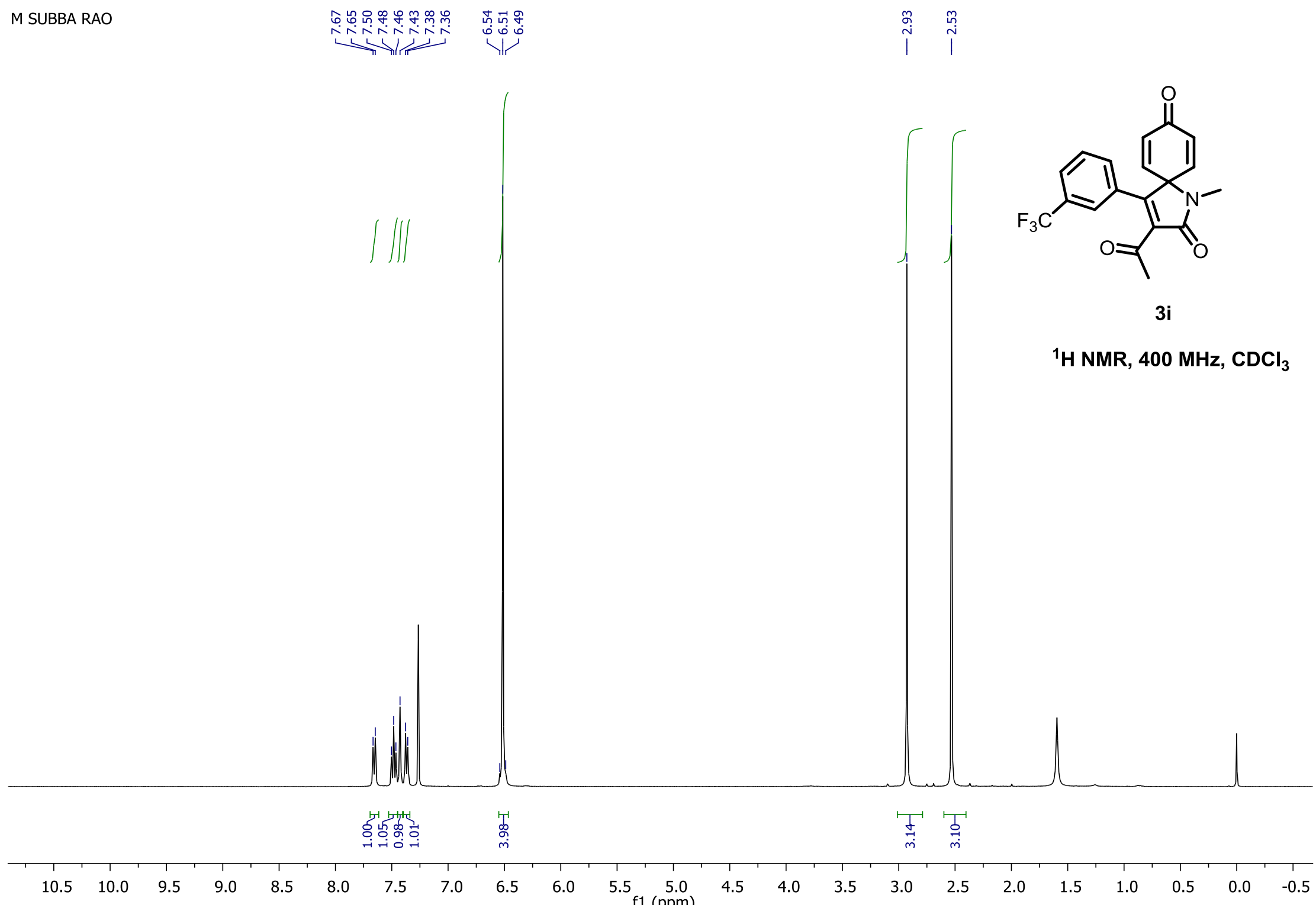




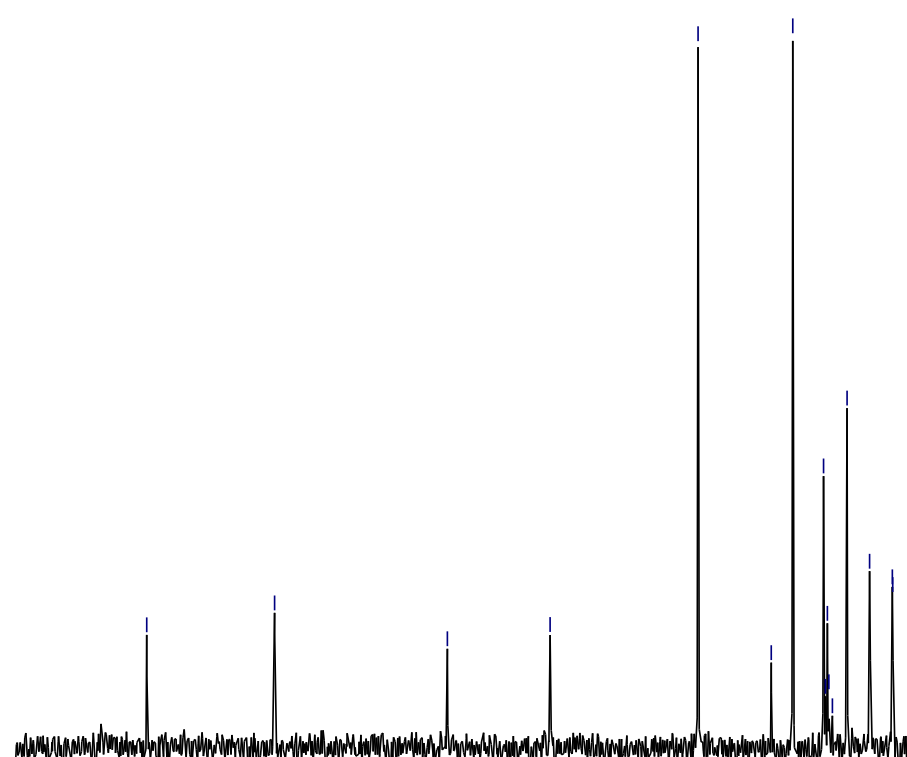

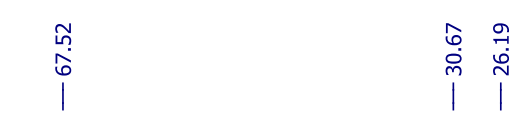

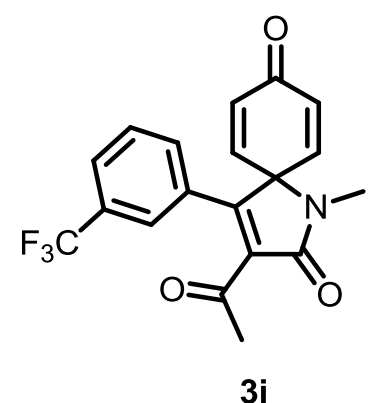

${ }^{13} \mathrm{C} \mathrm{NMR}, 101 \mathrm{MHz}, \mathrm{CDCl}_{3}$

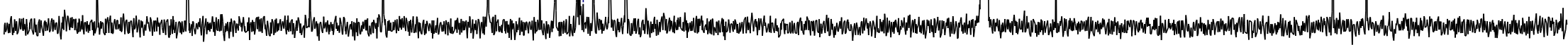
200
180
160
$150 \quad 140$
$130 \quad 120$
$110 \quad 100$
80
70
$60 \quad 50$
$40 \quad 30$
$20 \quad 10$ 


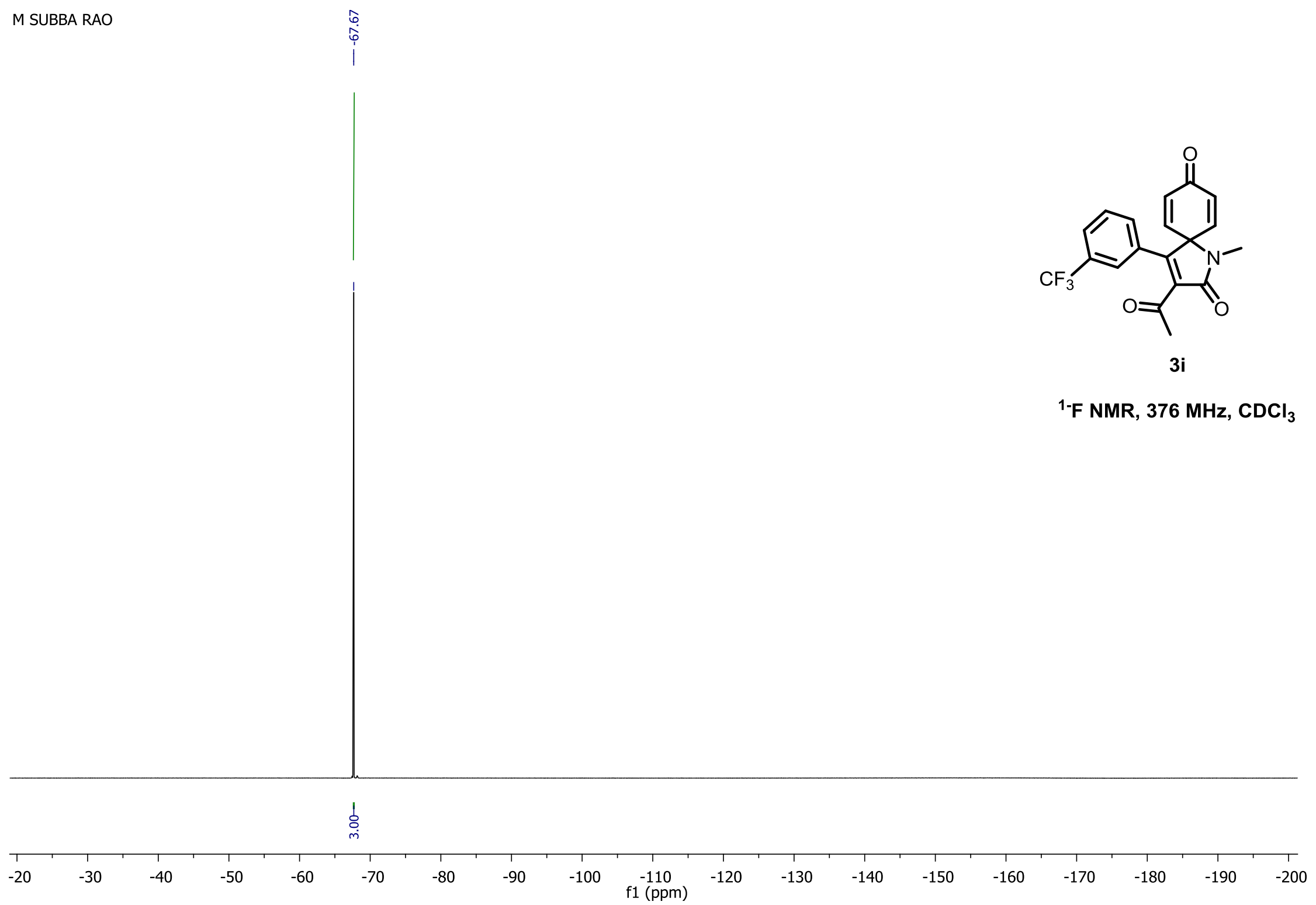



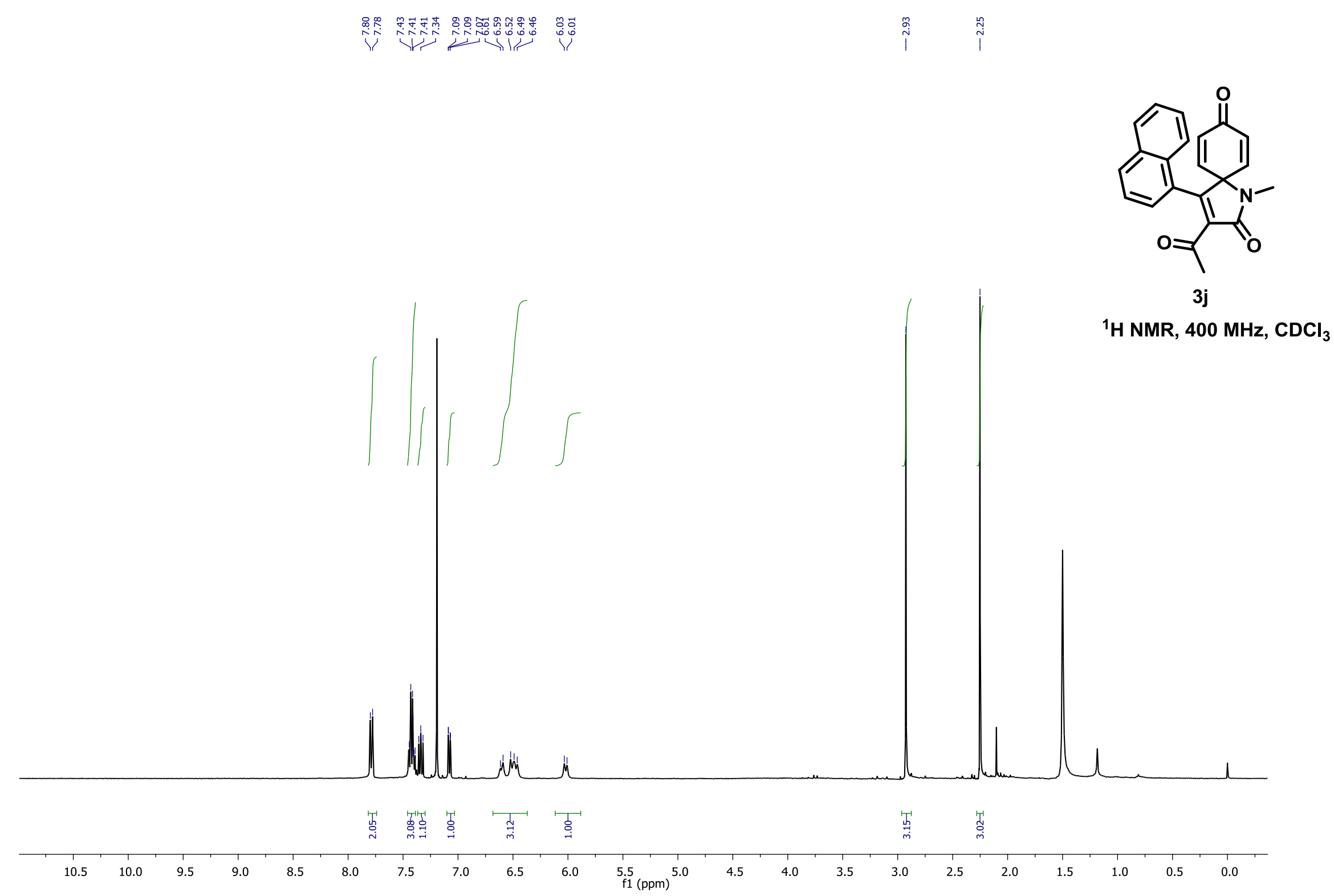


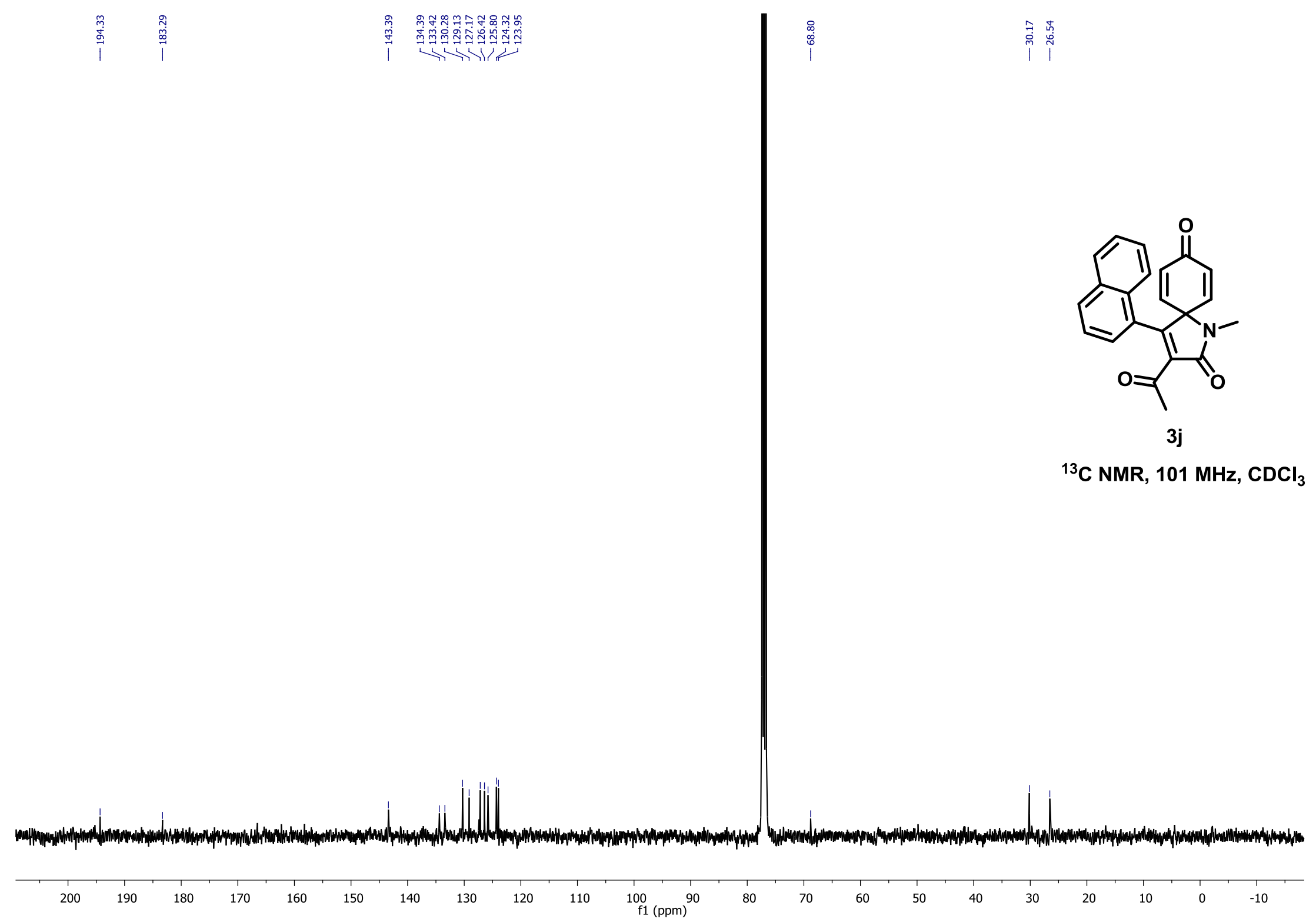




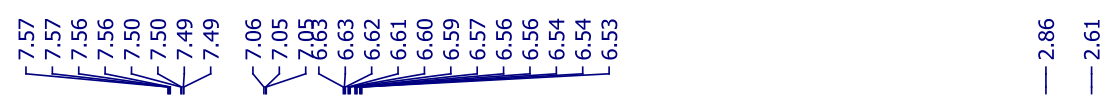

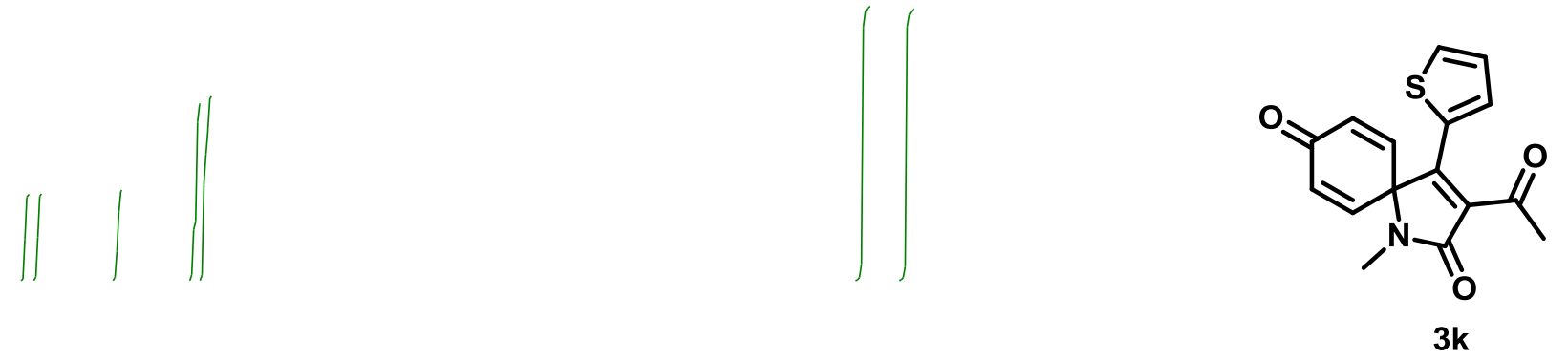

${ }^{1} \mathrm{H}$ NMR, $400 \mathrm{MHz}, \mathrm{CDCl}_{3}$

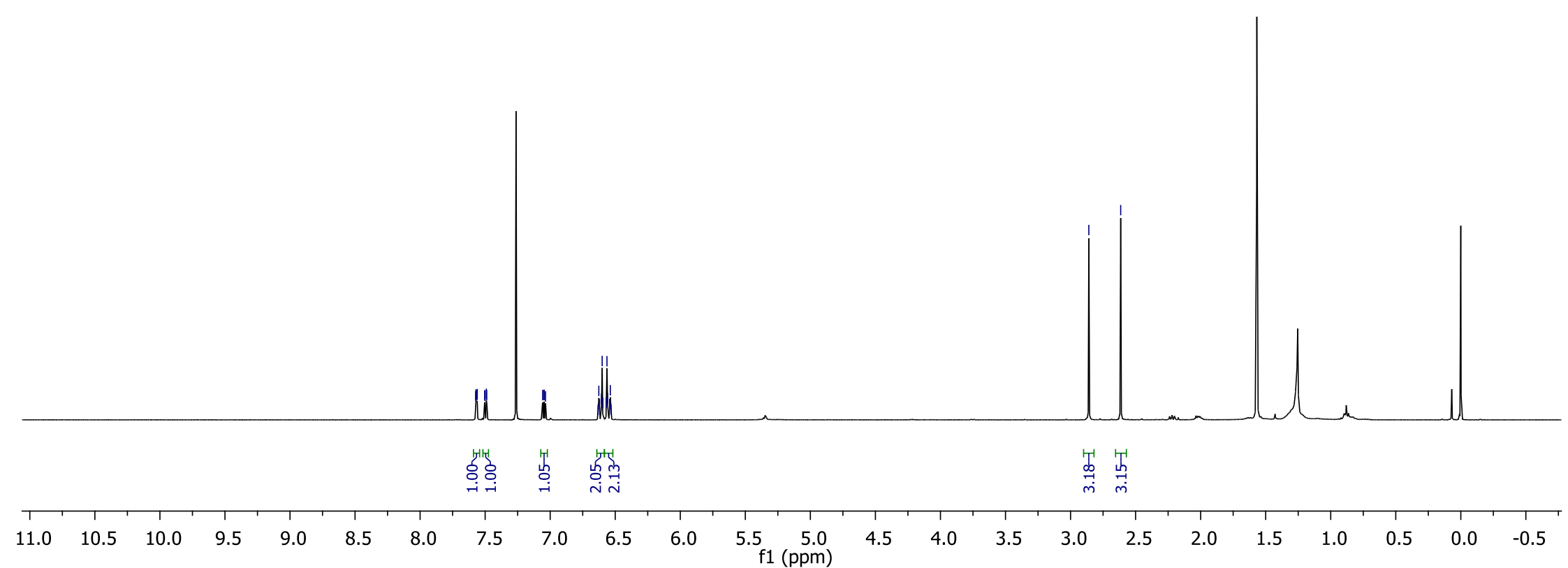




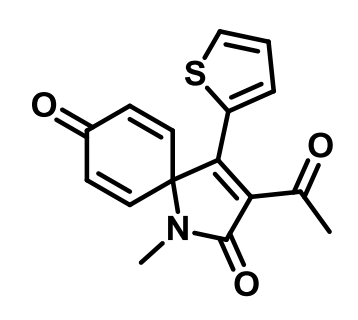

${ }^{13} \mathrm{C}$ NMR, $101 \mathrm{MHz}, \mathrm{CDCl}_{3}$

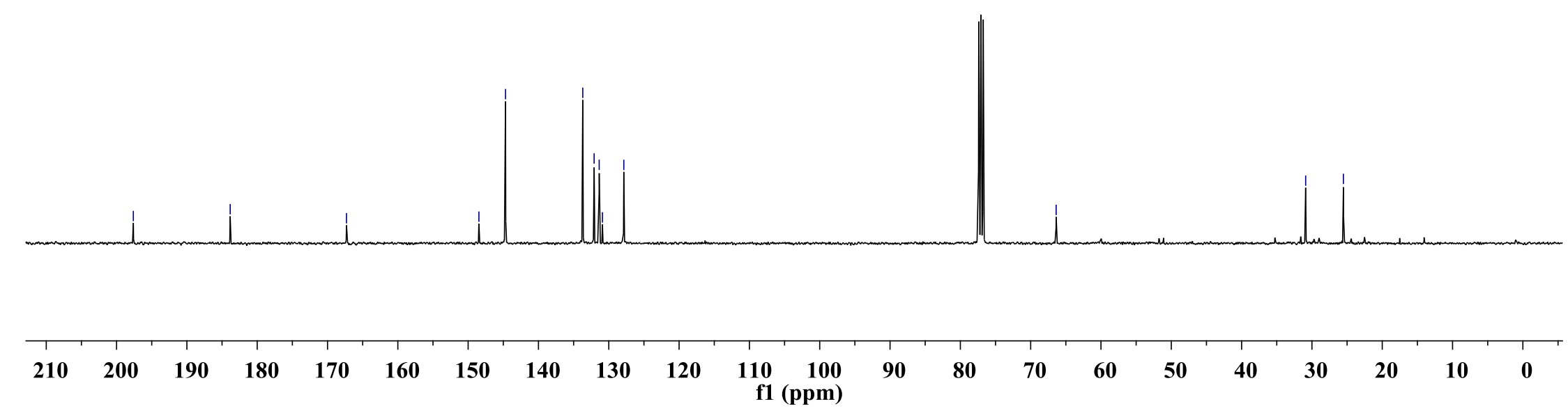




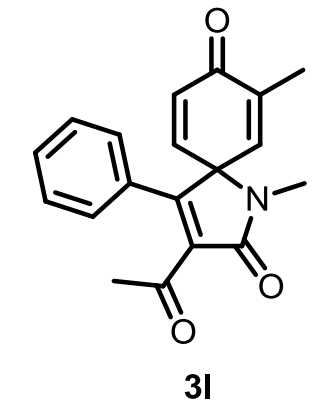

${ }^{1} \mathrm{H} \mathrm{NMR,}, 400 \mathrm{MHz}, \mathrm{CDCl}_{3}$

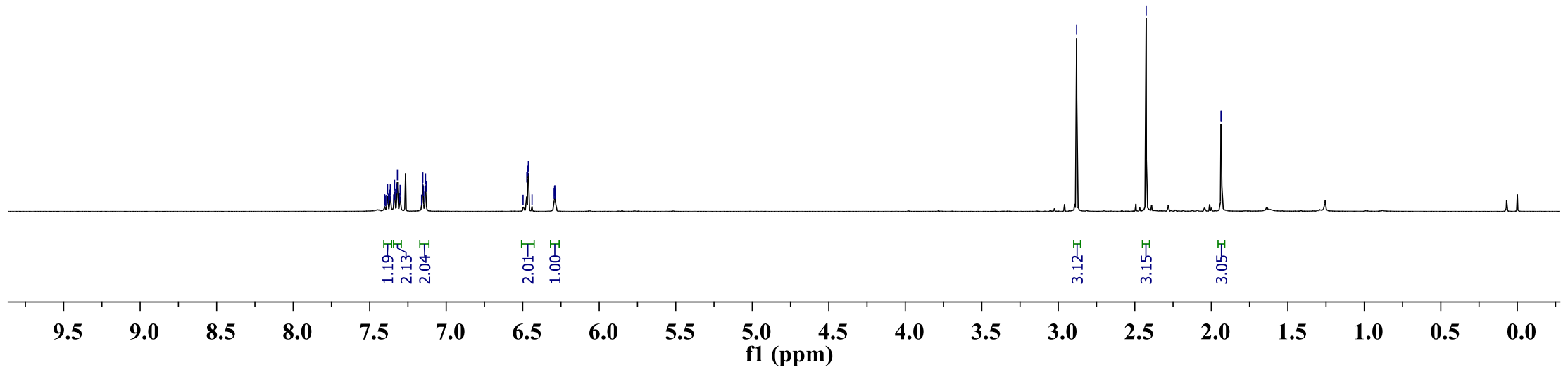




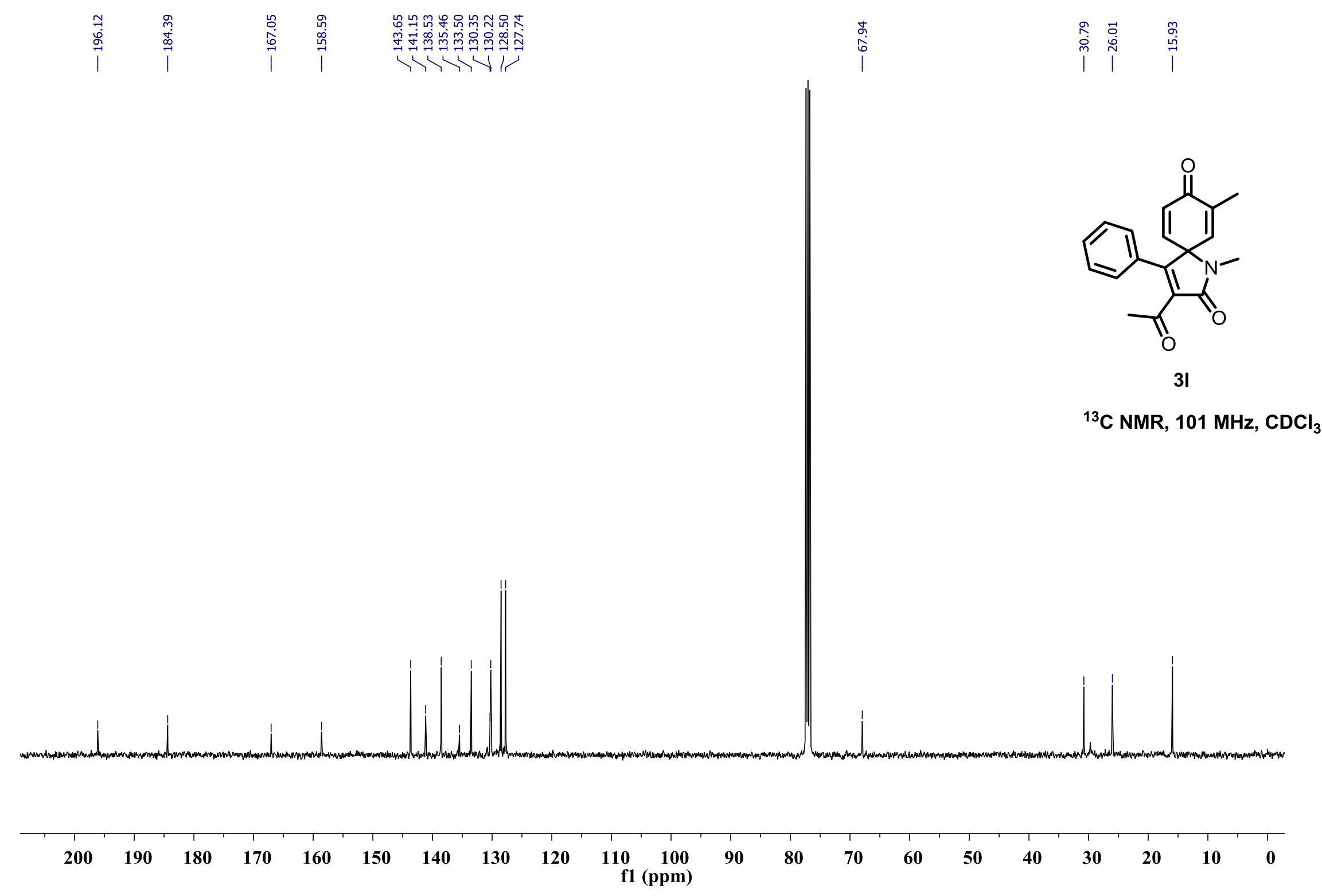




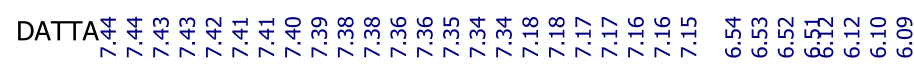

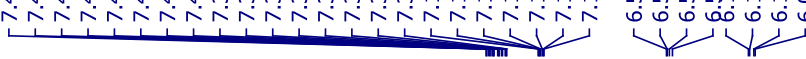

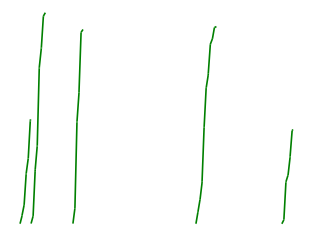

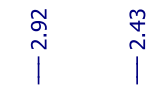
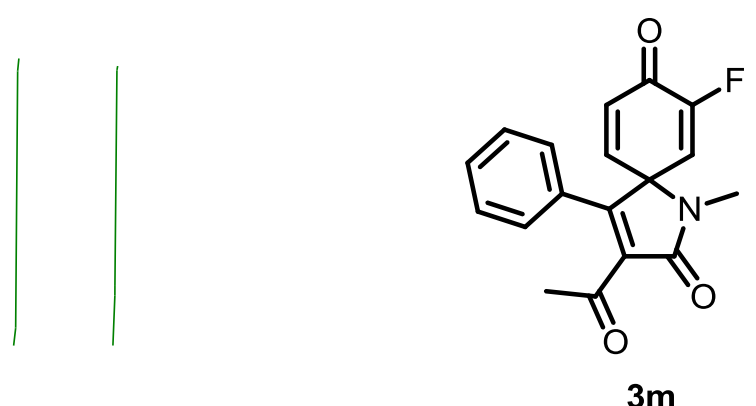

${ }^{1} \mathrm{H}$ NMR, $400 \mathrm{MHz}, \mathrm{CDCl}_{3}$

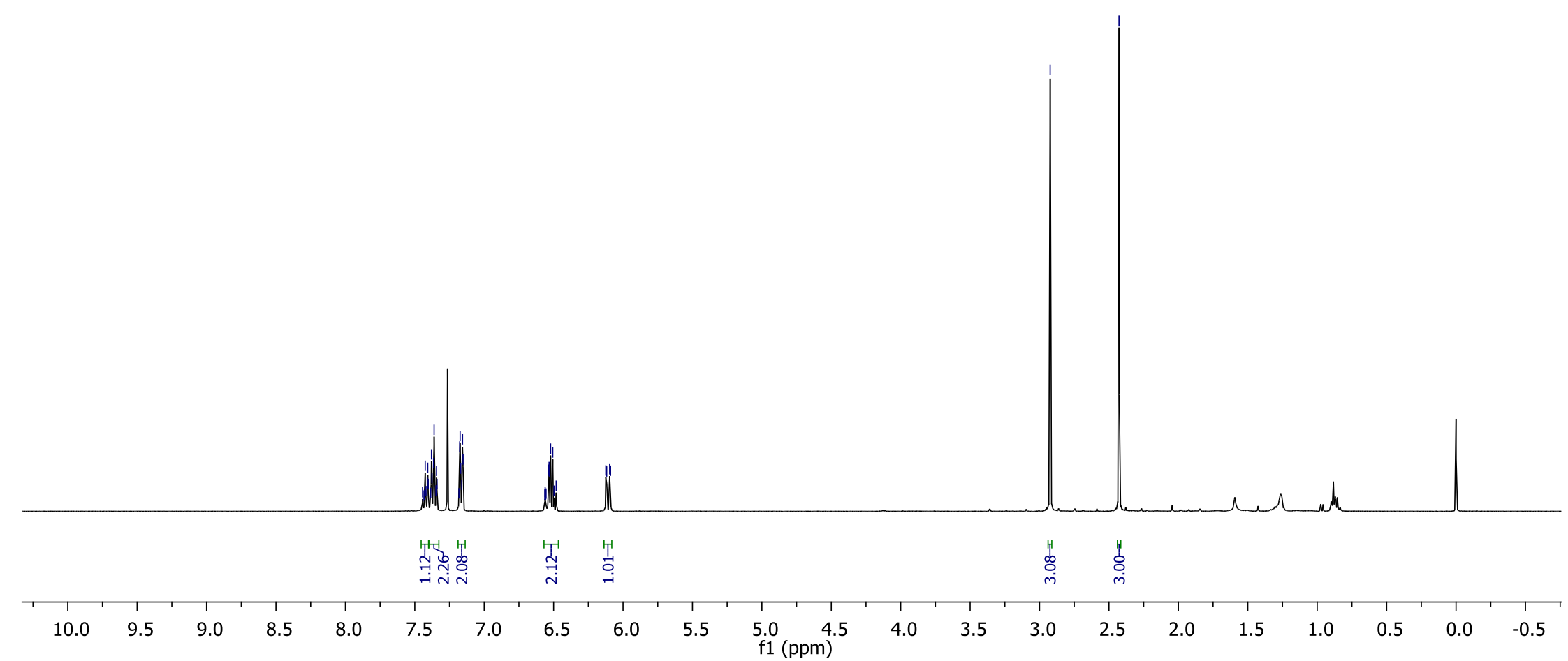



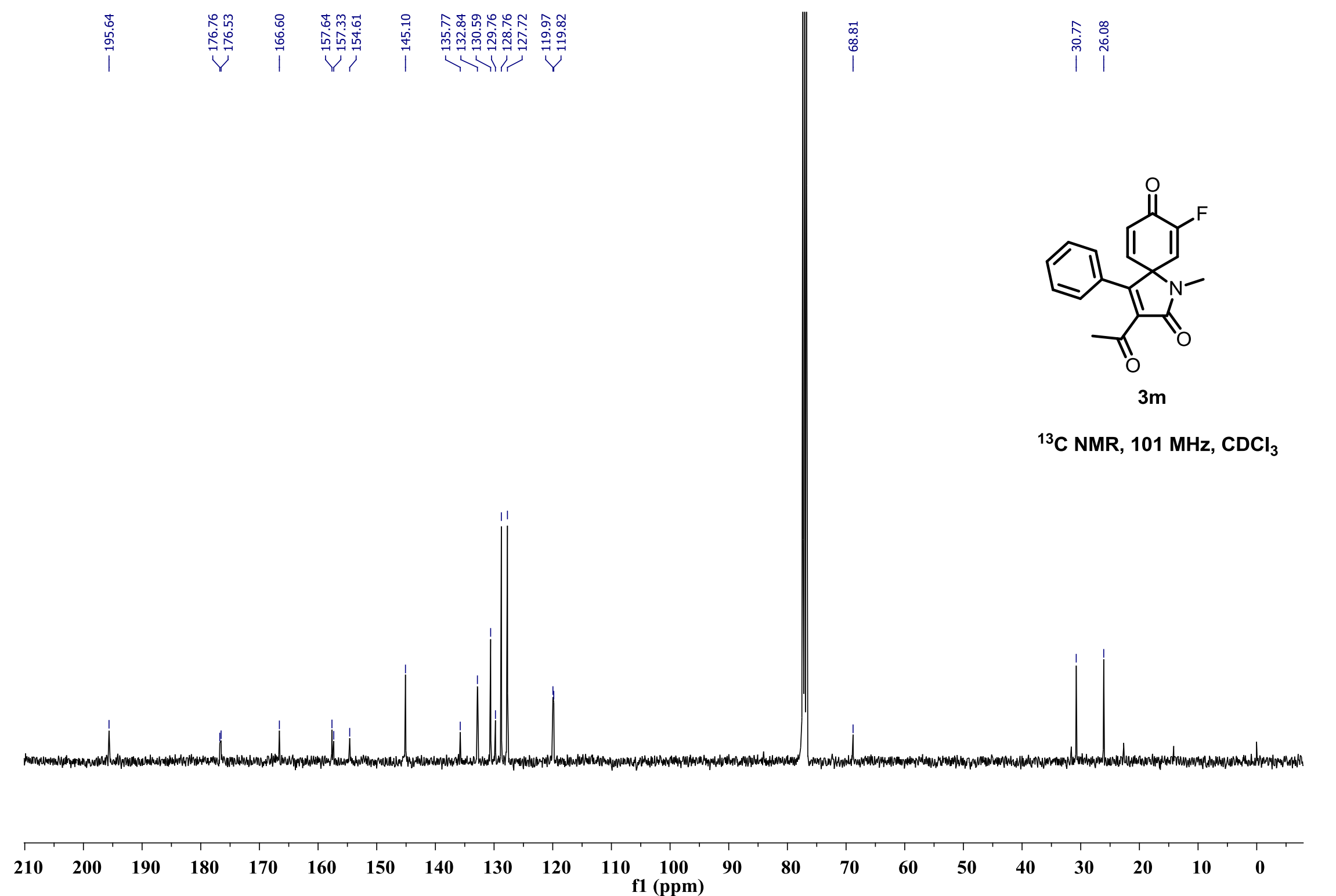
M SUBBA RAO

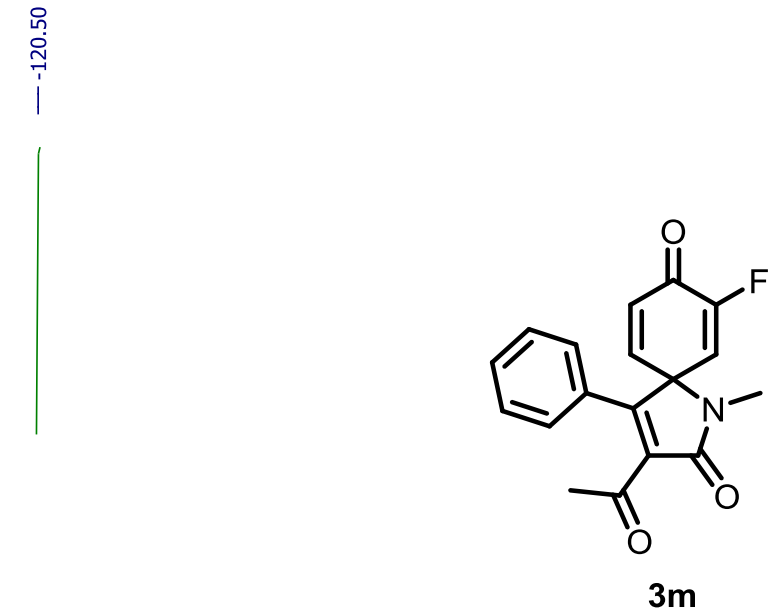

${ }^{19} \mathrm{~F}$ NMR, $375 \mathrm{MHz}^{\mathrm{CDCl}}{ }_{3}$

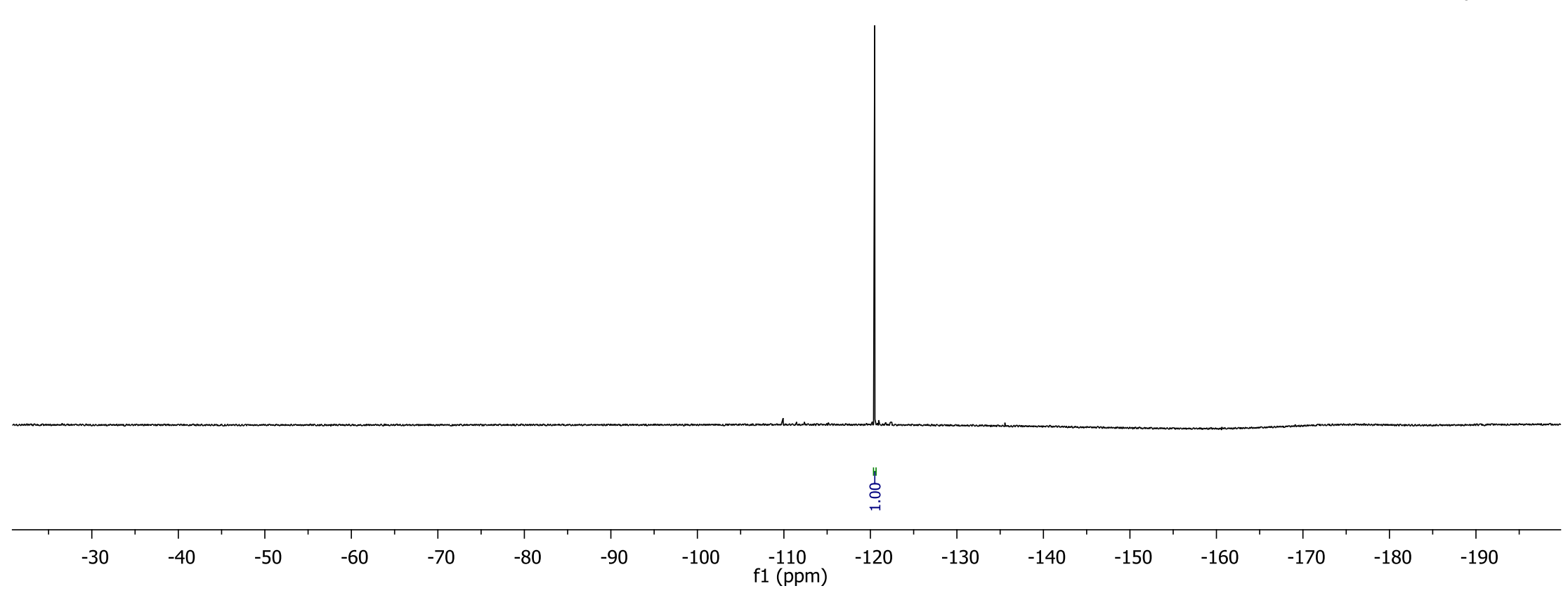



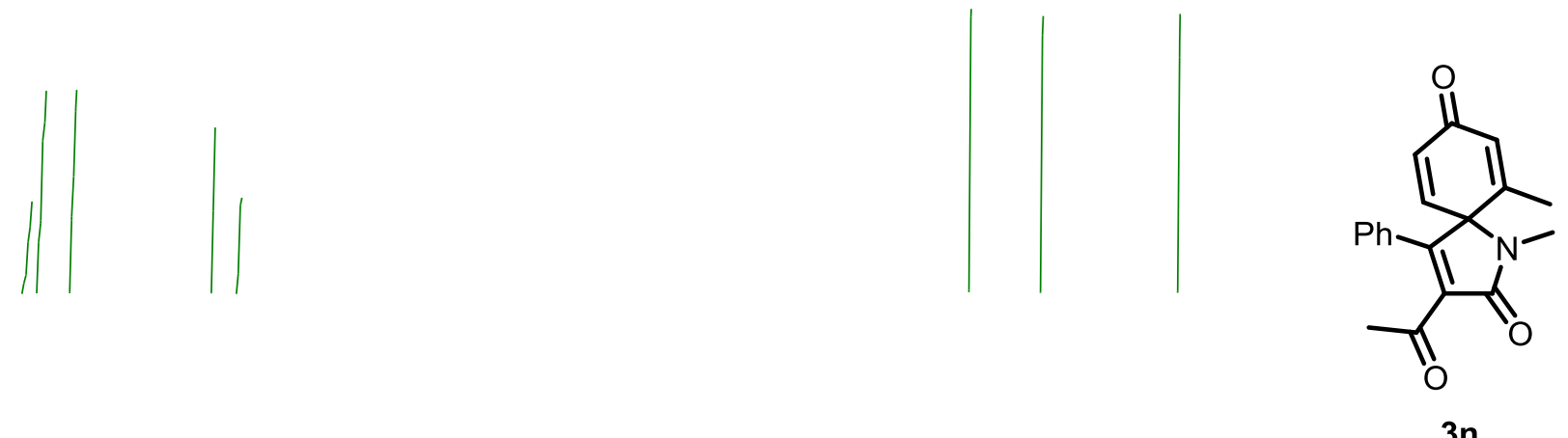

${ }^{1} \mathrm{HNMR}, 400 \mathrm{MHz}, \mathrm{CDCl}_{3}$

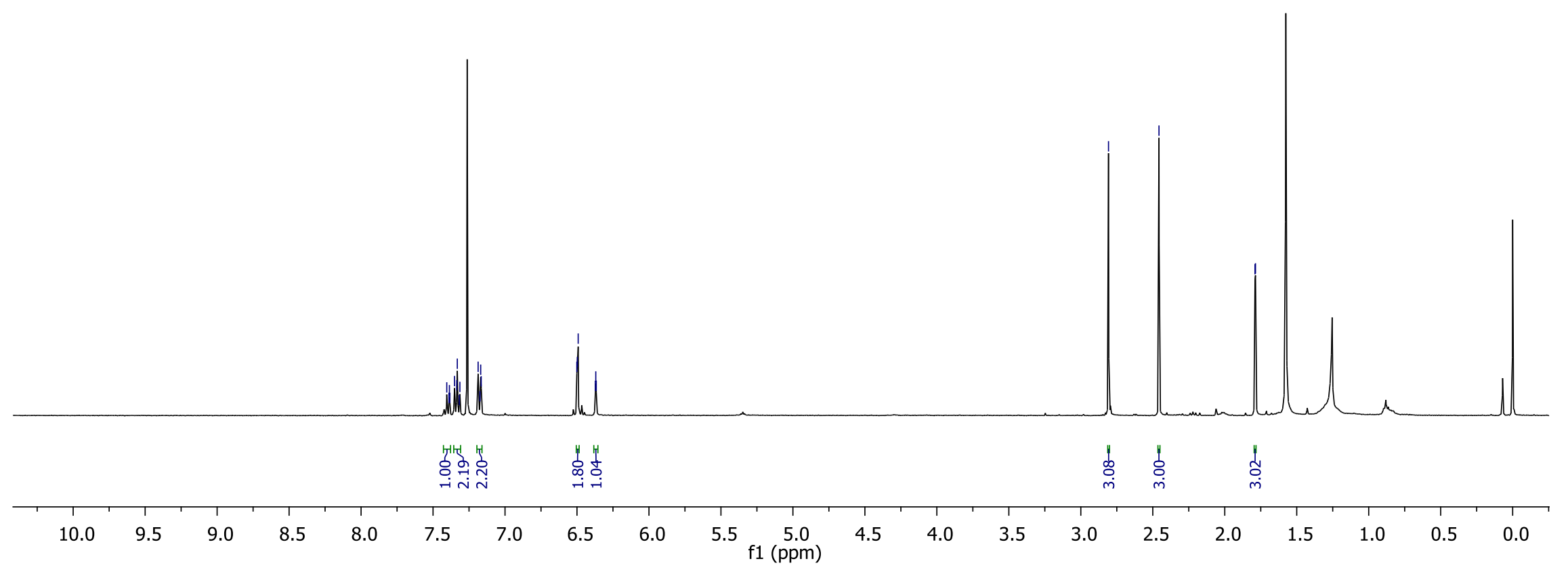




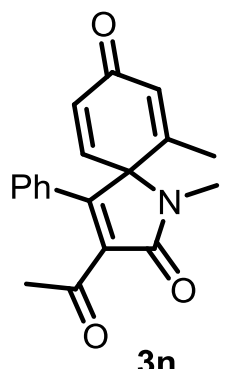

${ }^{13} \mathrm{CNMR}, 100 \mathrm{MHz}, \mathrm{CDCl}_{3}$
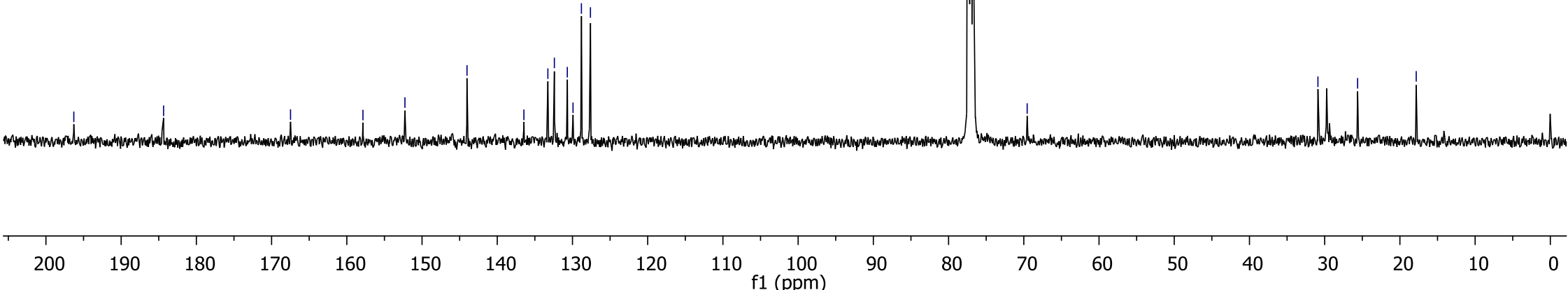


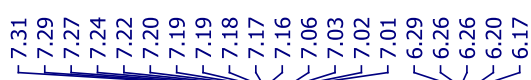
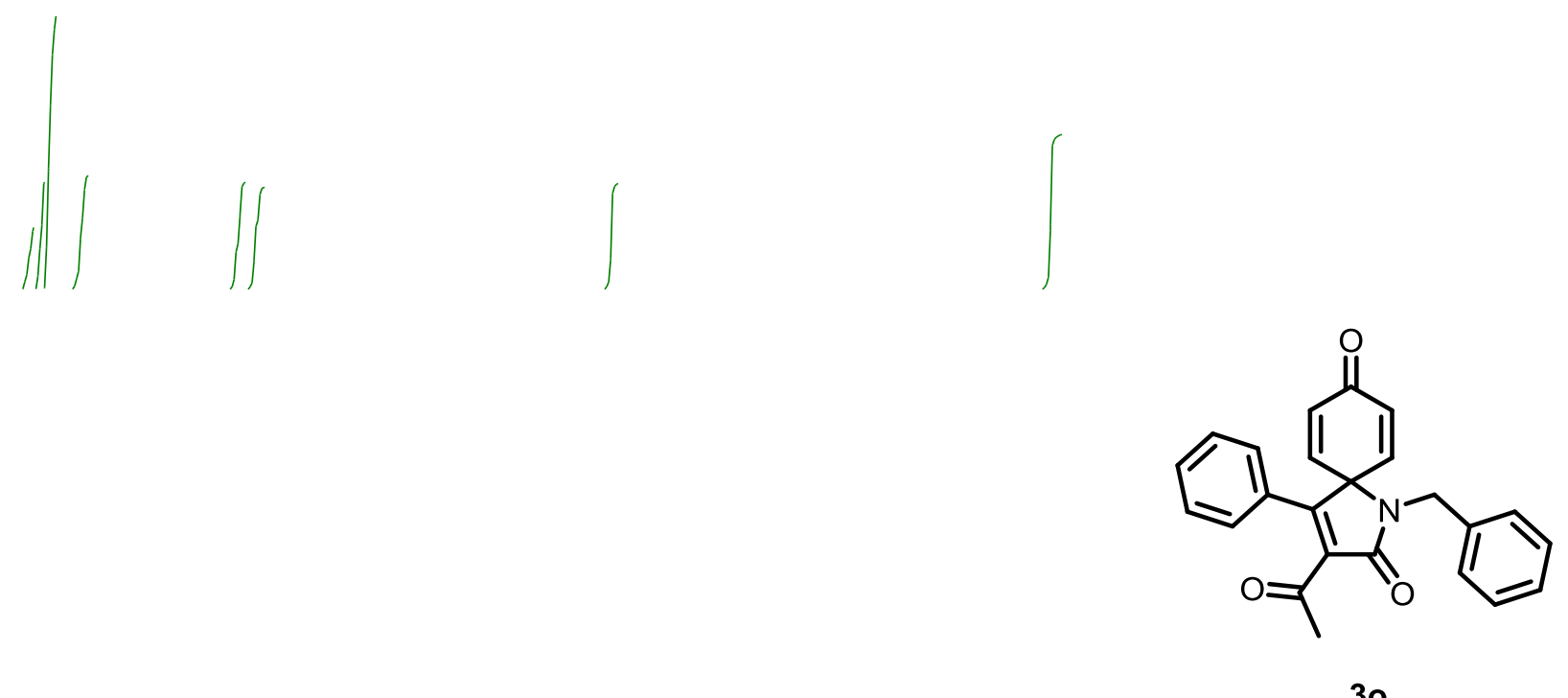

${ }^{1} \mathrm{H}$ NMR, $400 \mathrm{MHz} \mathrm{CDCl}_{3}$

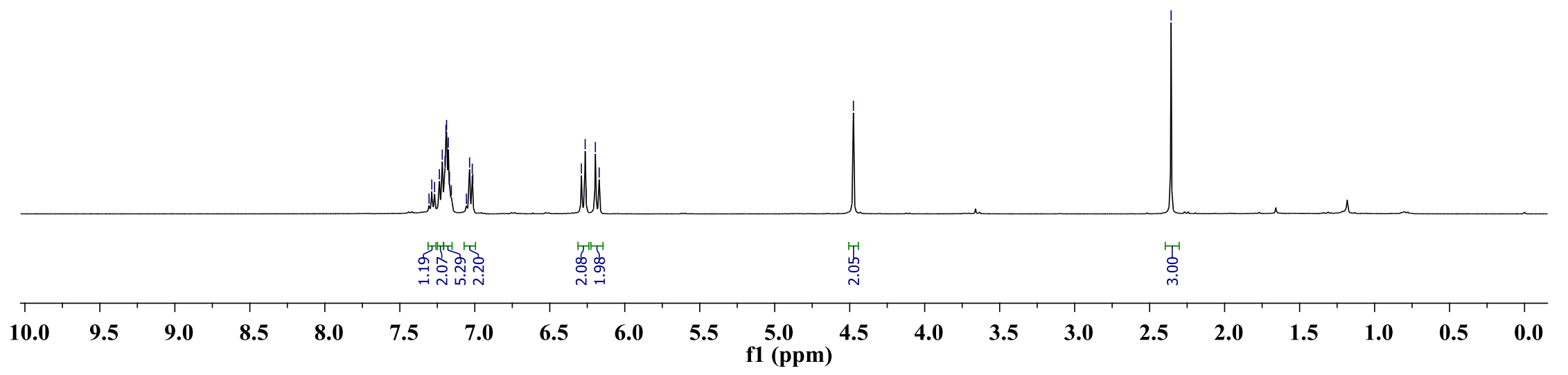




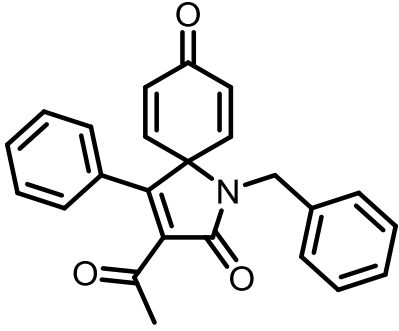

30

${ }^{13} \mathrm{C}$ NMR, $101 \mathrm{MHz}, \mathrm{CDCl}_{3}$

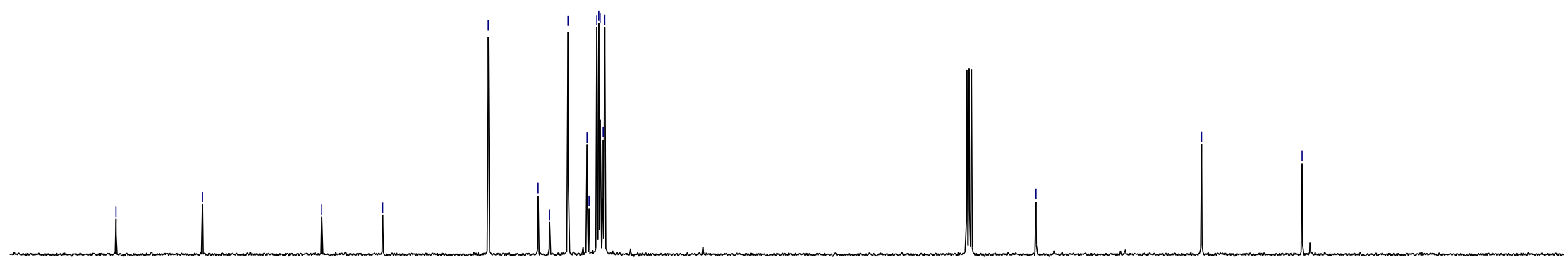




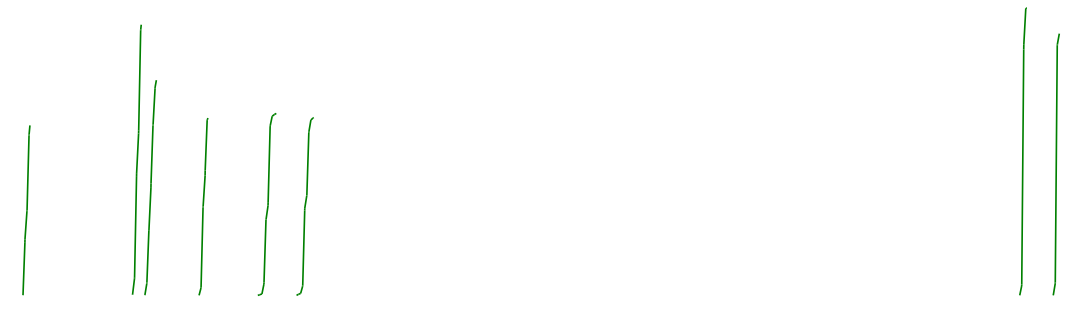

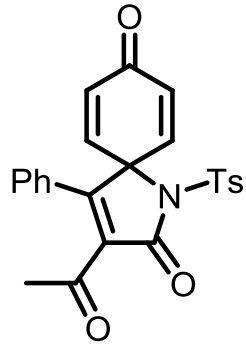

$3 p$

${ }^{1}$ HNMR, 400MHz, $\mathrm{CDCl}_{3}$

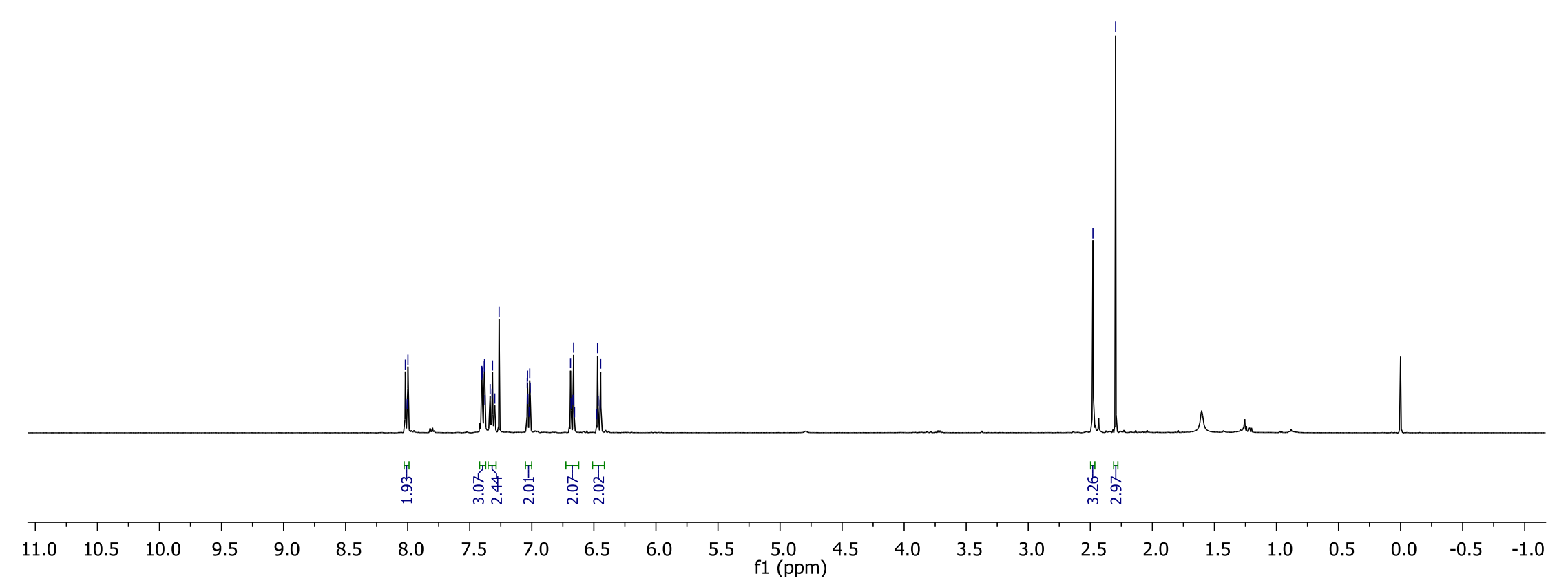




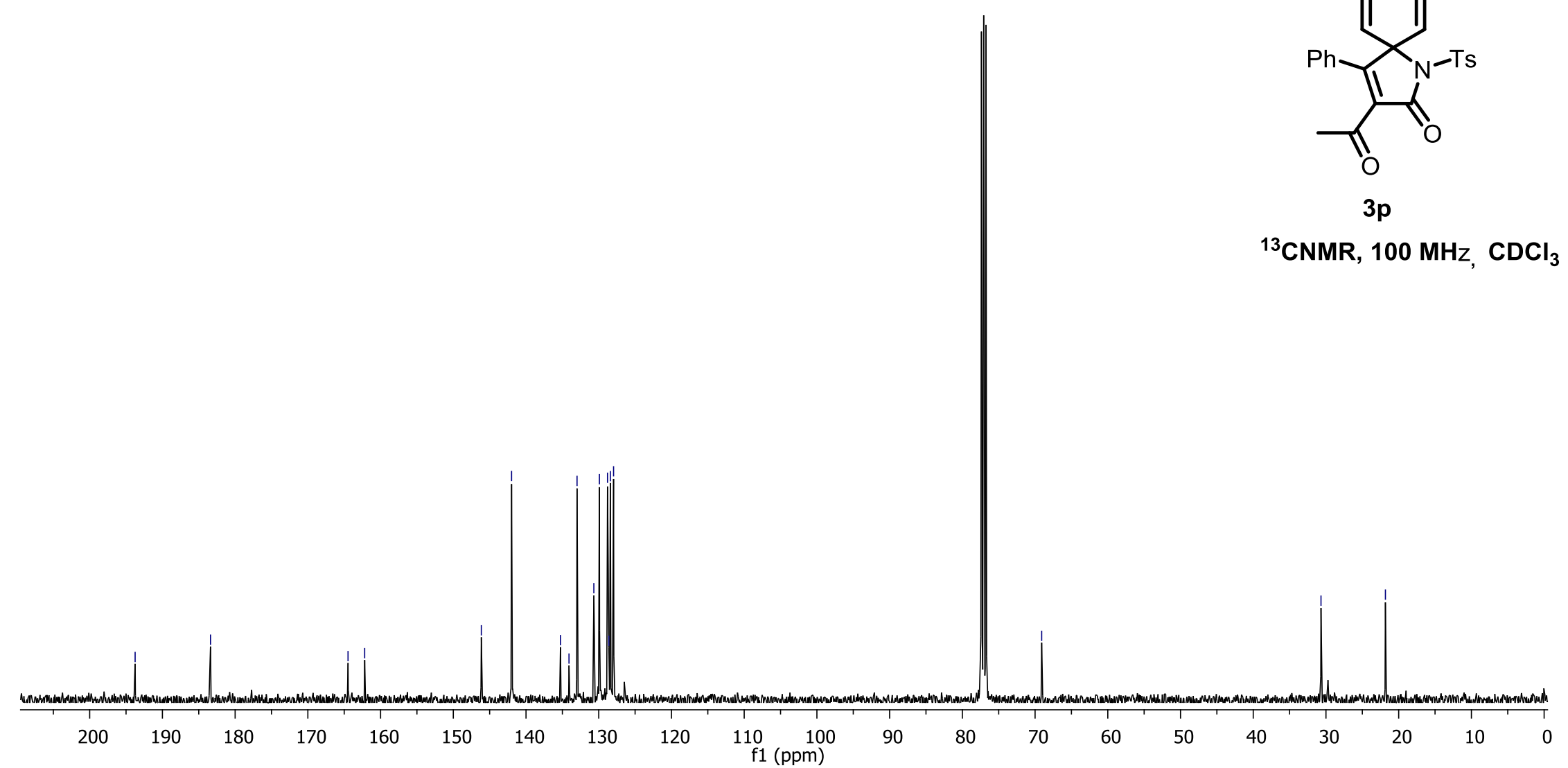




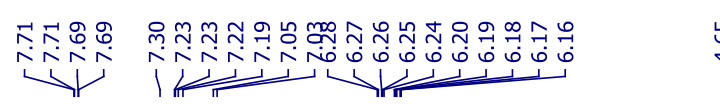

总
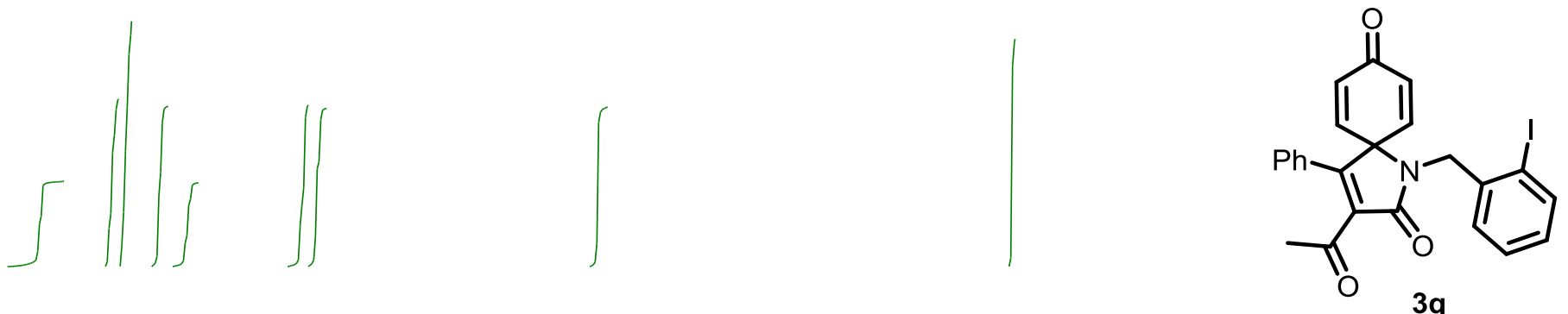

${ }^{1} \mathrm{H}$ NMR, $400 \mathrm{MHz}, \mathrm{CDCl}_{3}$

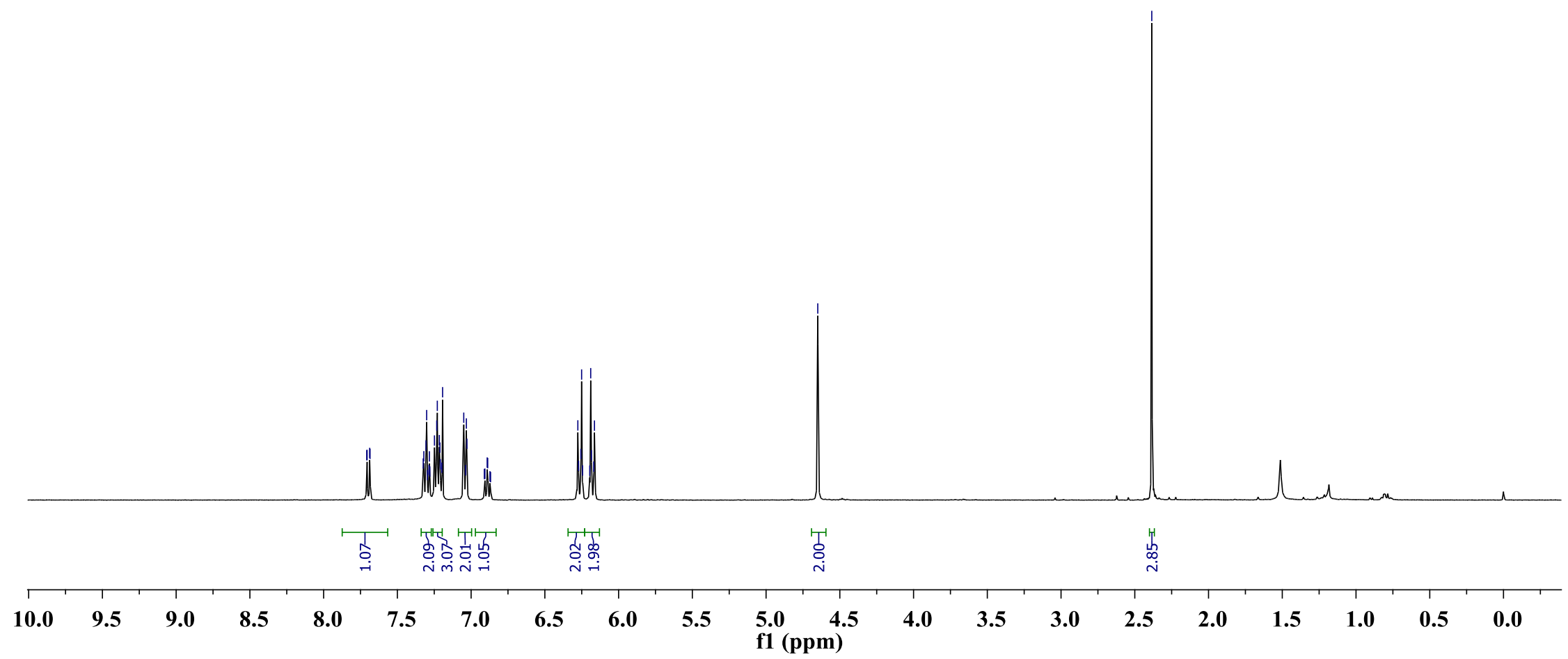




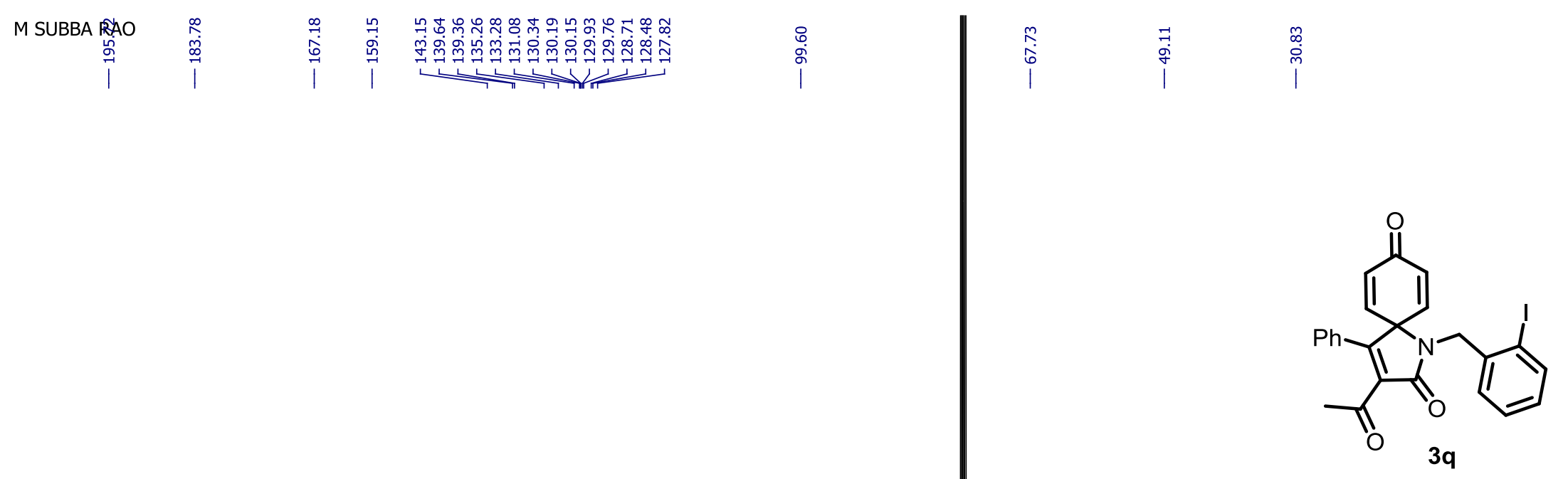

${ }^{13} \mathrm{C} \mathrm{NMR}, 100 \mathrm{MHz}, \mathrm{CDCl}_{3}$

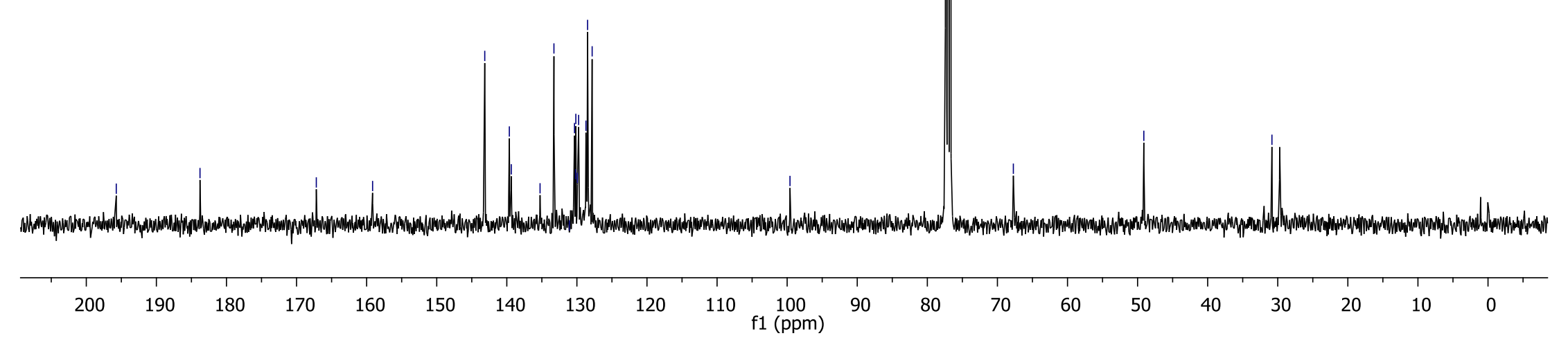




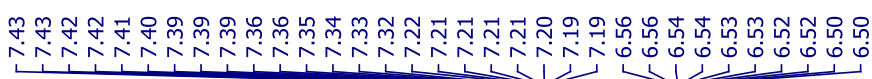

ํำ

$\sqrt{1 / 4}$

îjo
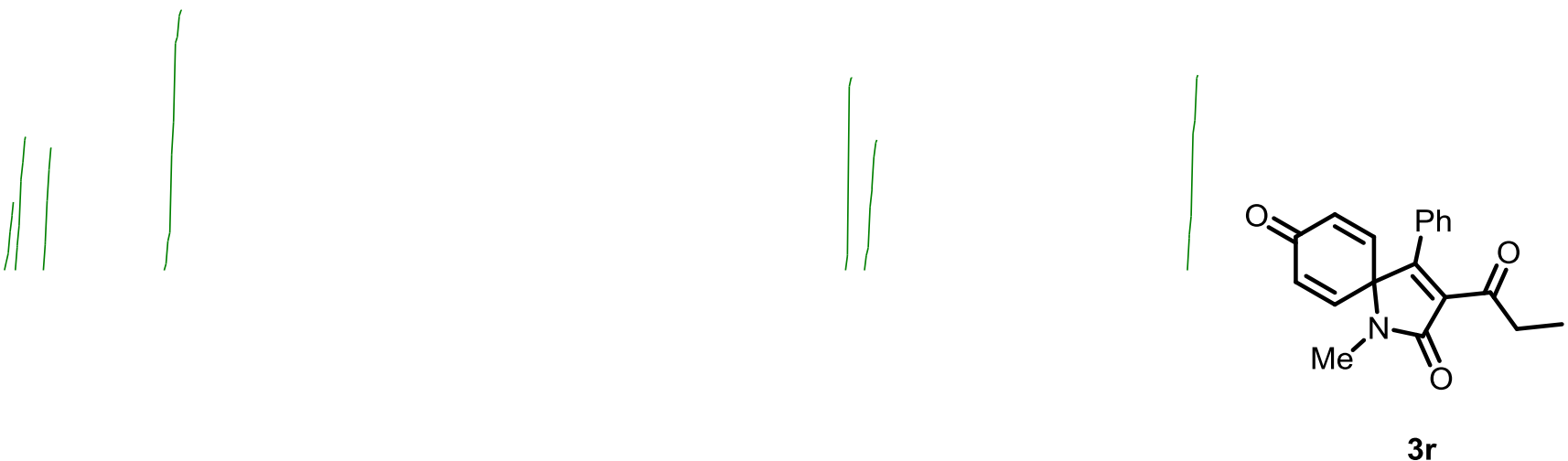

${ }^{1} \mathrm{H}$ NMR, $400 \mathrm{MHz}, \mathrm{CDCl}_{3}$

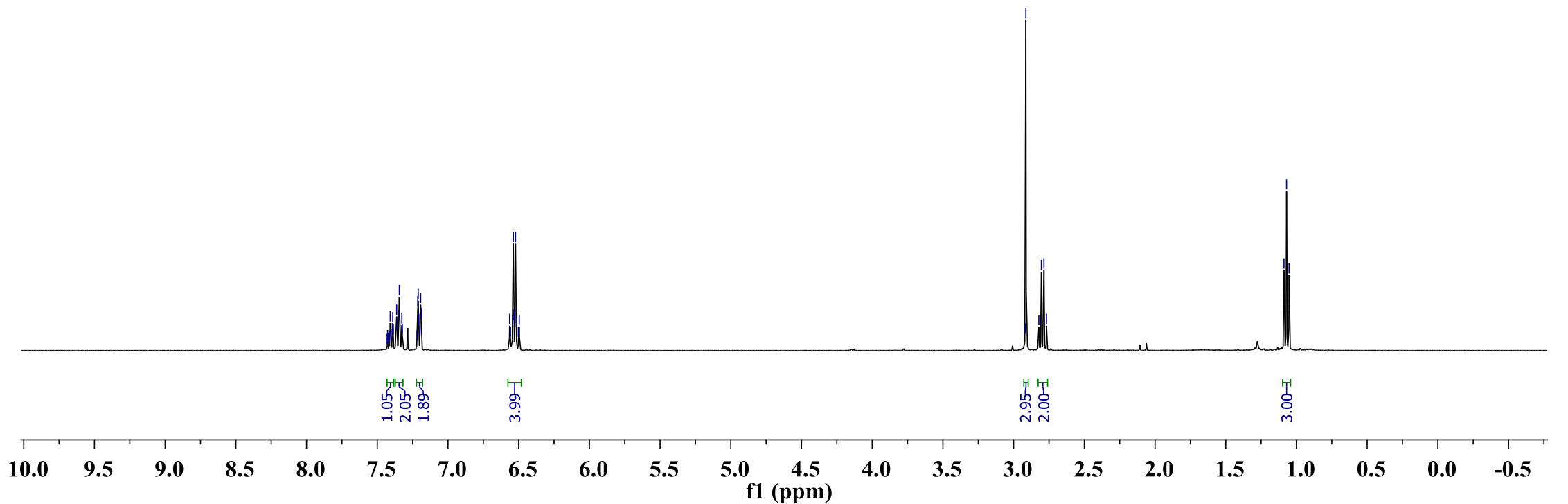




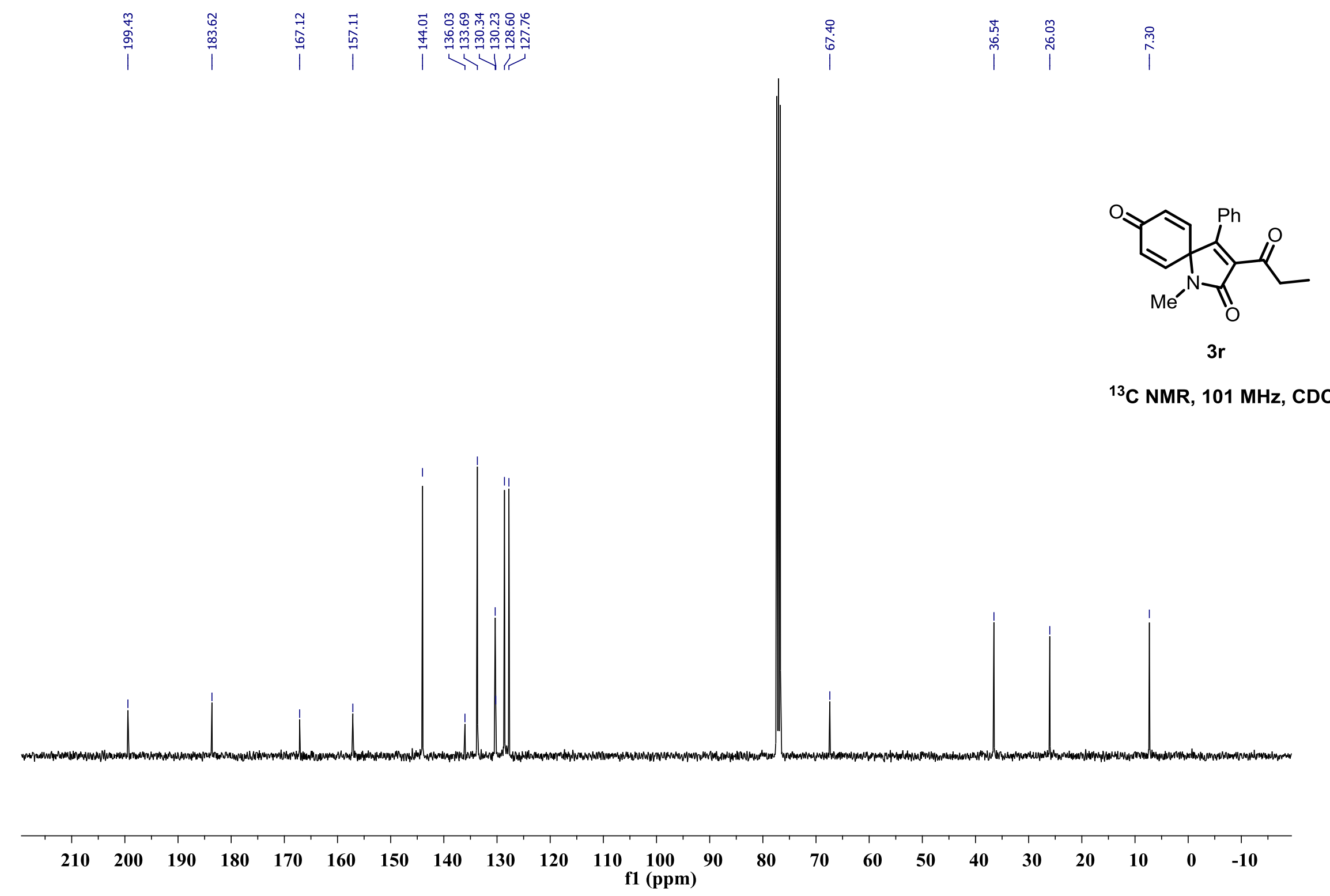




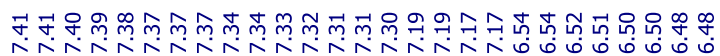

(1)

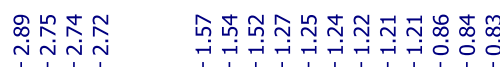

西
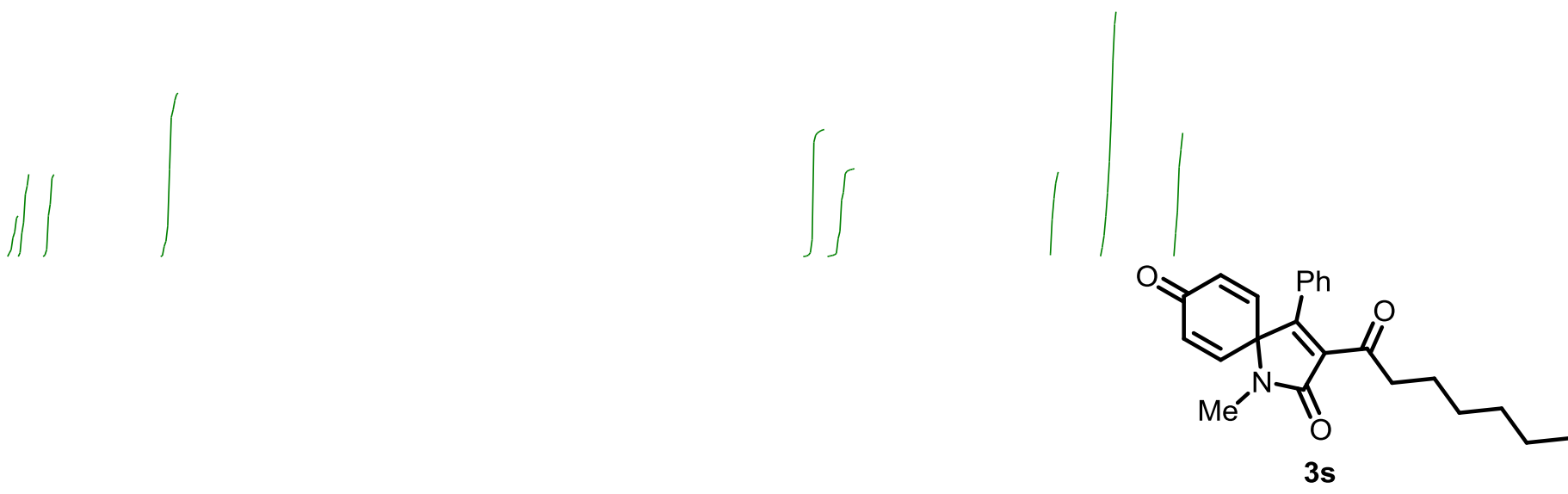

${ }^{1} \mathrm{H}$ NMR, $400 \mathrm{MHz}^{\mathrm{CDCl}}{ }_{3}$

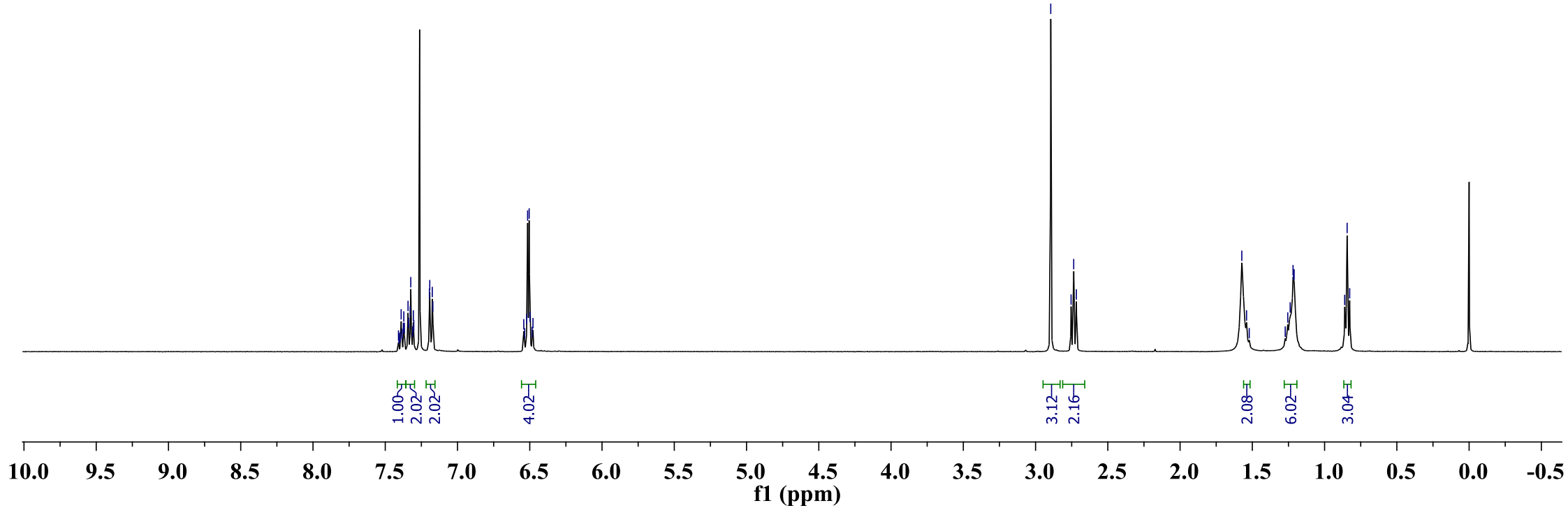




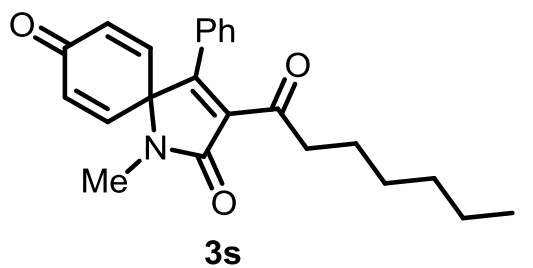

${ }^{13} \mathrm{C}$ NMR, $100 \mathrm{MHz}^{\mathrm{CDCl}} \mathrm{C}_{3}$

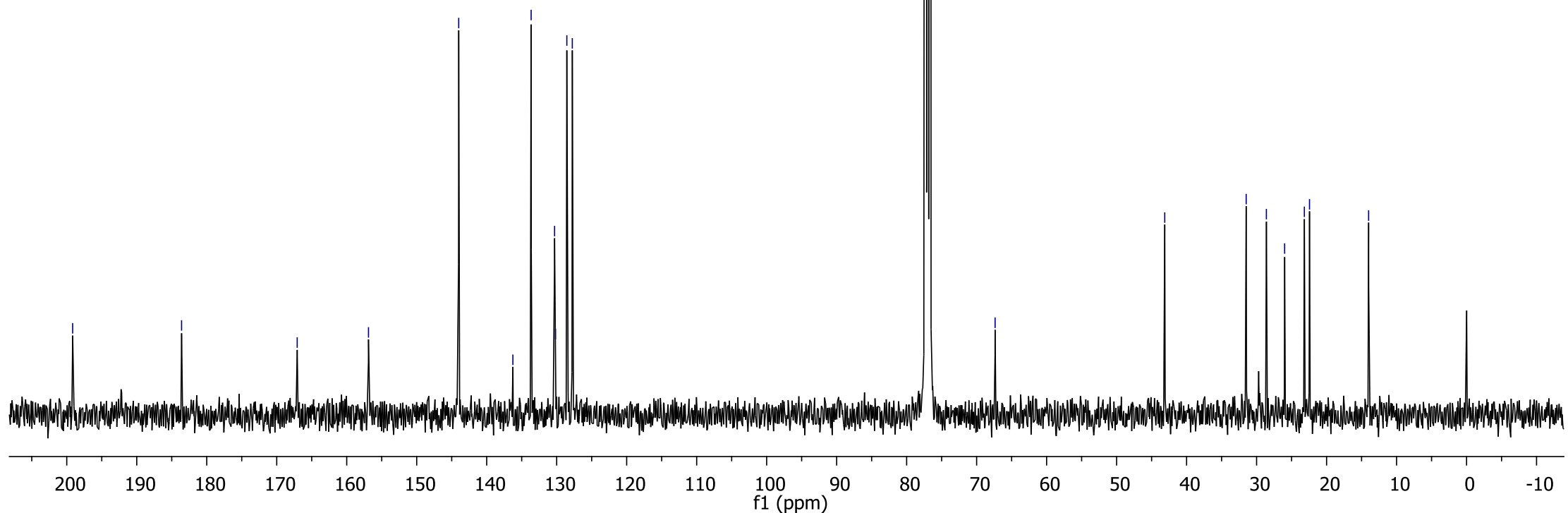




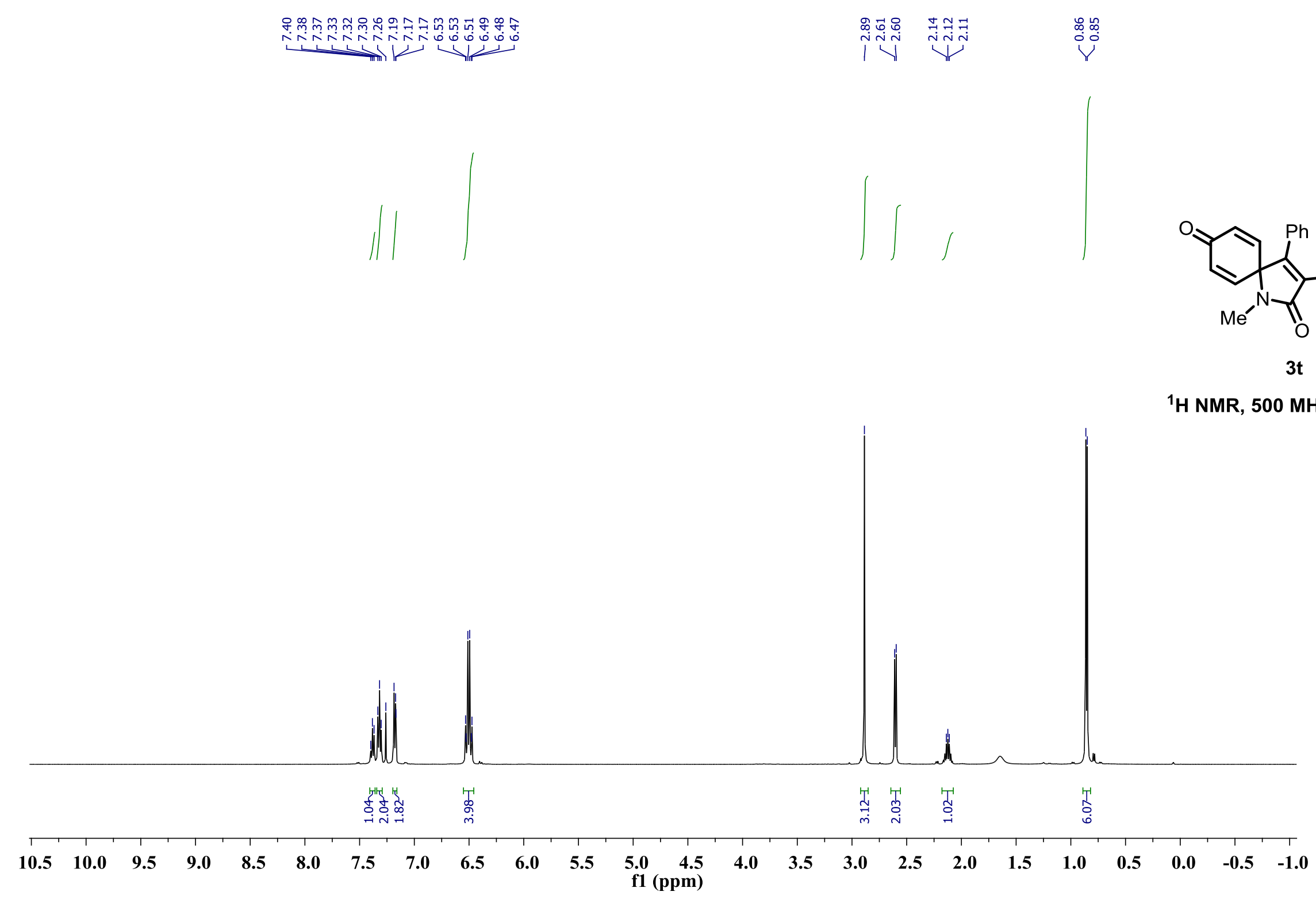



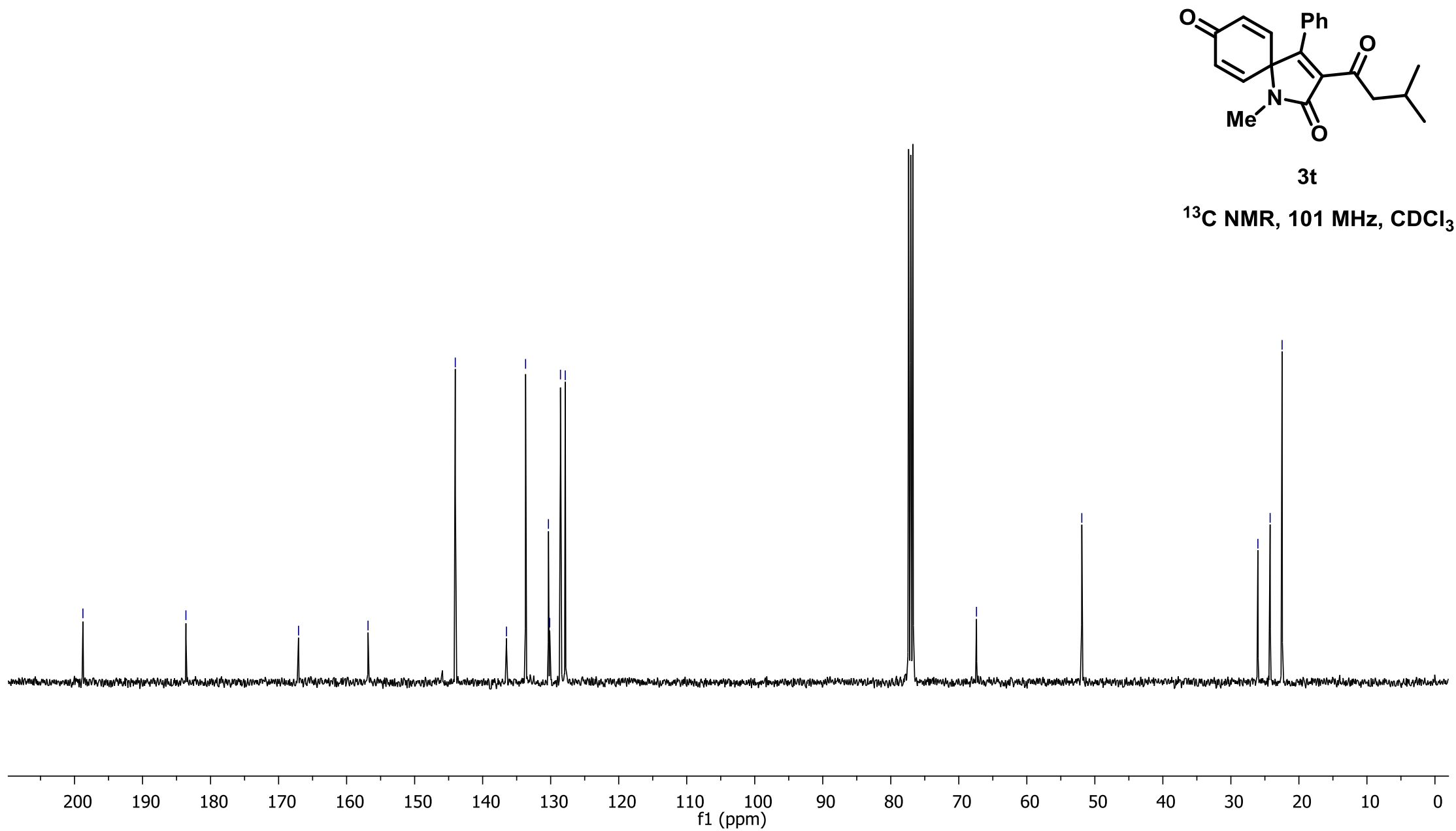


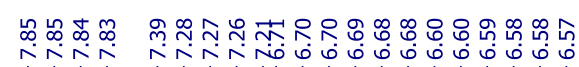

N 1
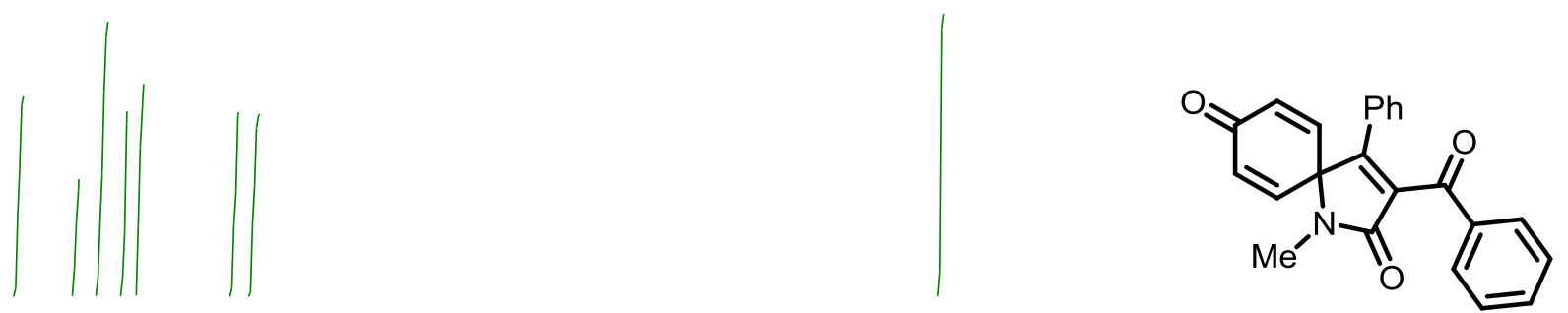

3u

${ }^{1} \mathrm{H}$ NMR, $500 \mathrm{MHz}, \mathrm{CDCl}_{3}$

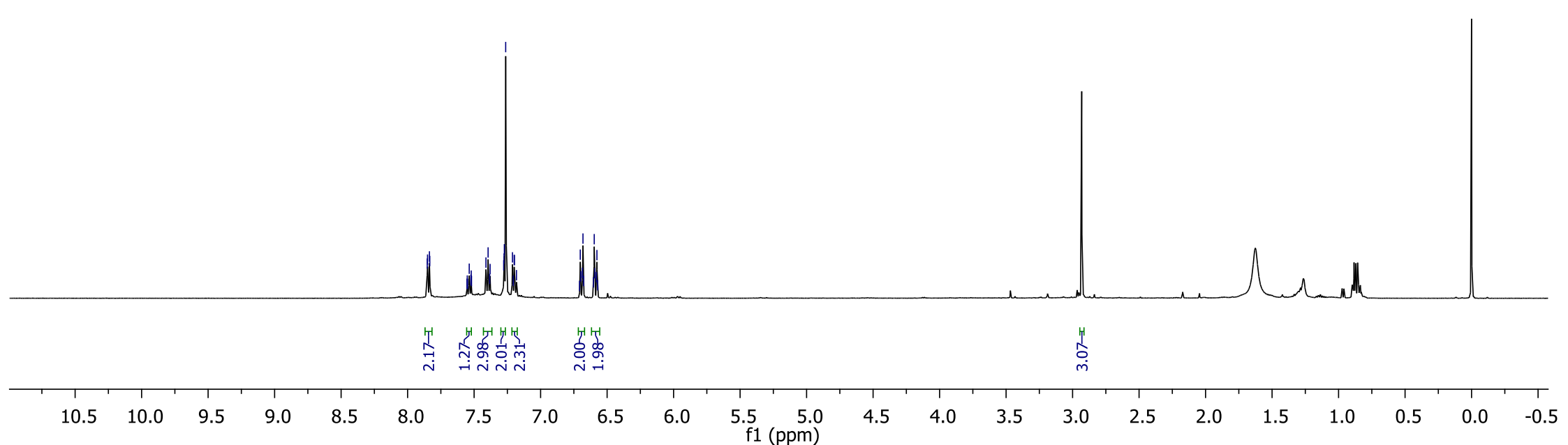




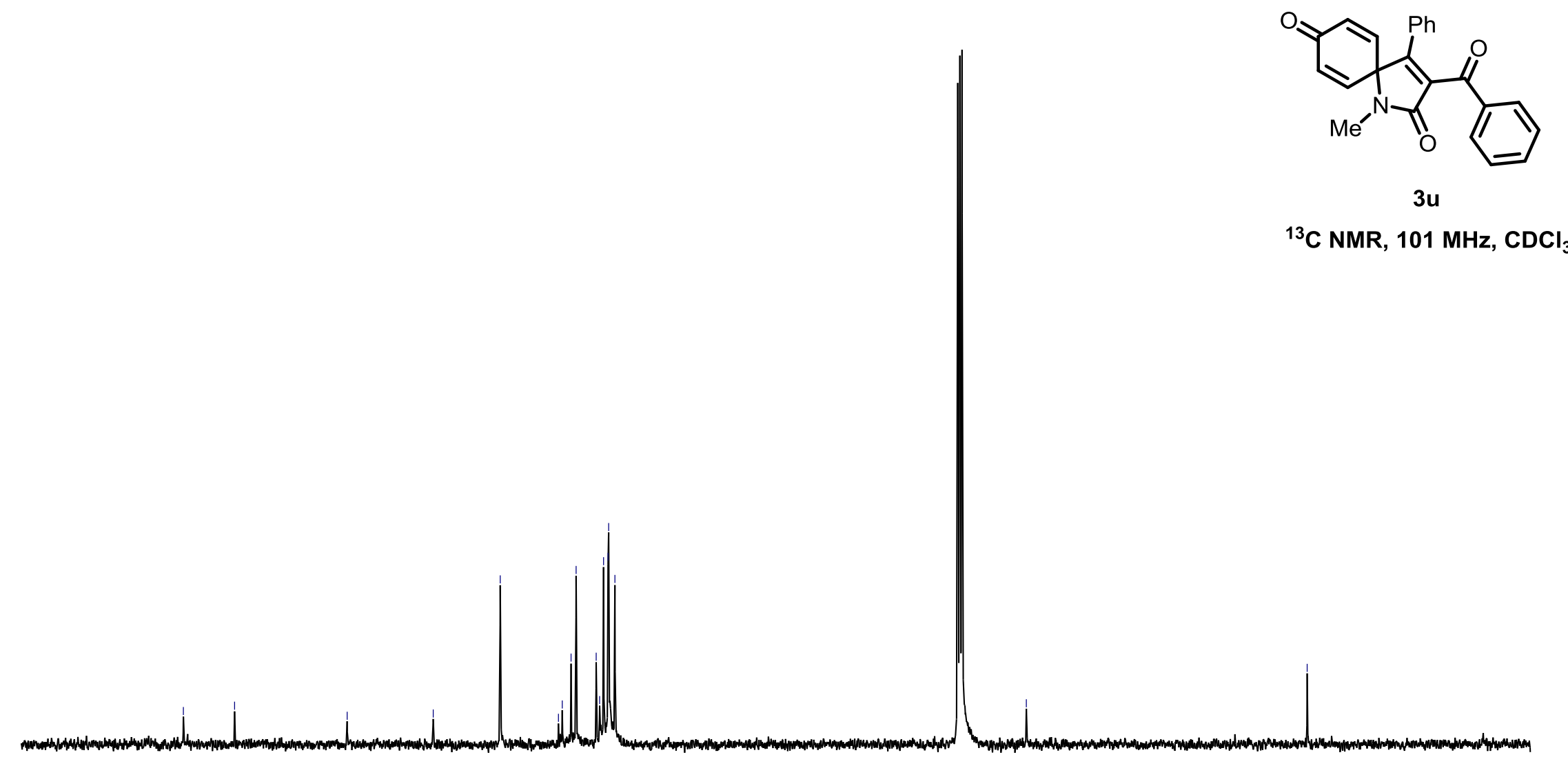

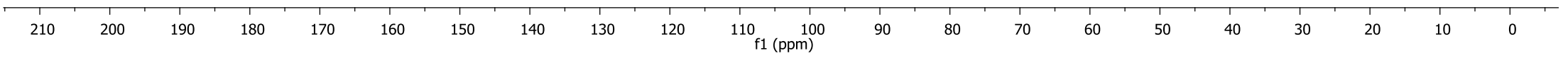



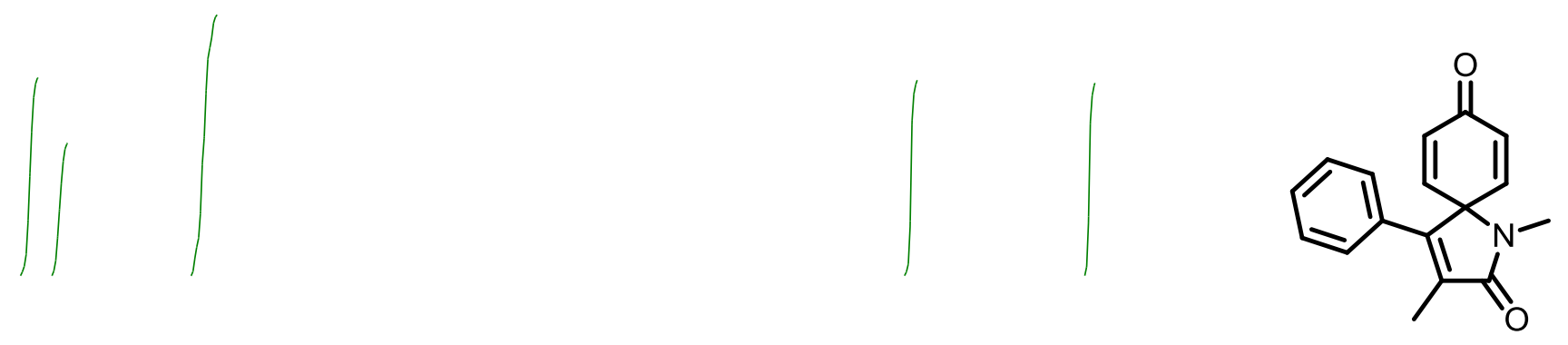

$5 a$

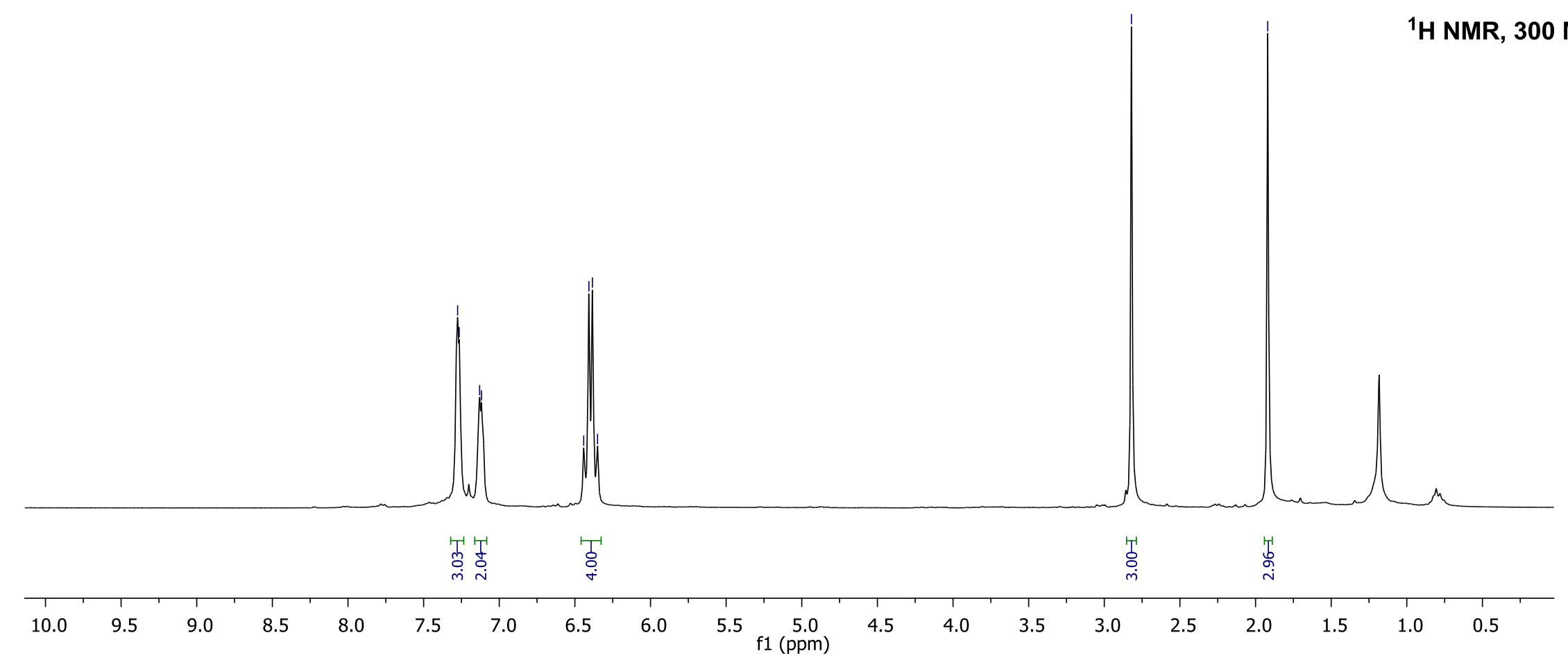




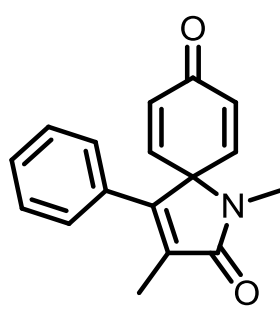

$5 a$

${ }^{13} \mathrm{C}$ NMR, $101 \mathrm{MHz}, \mathrm{CDCl}_{3}$

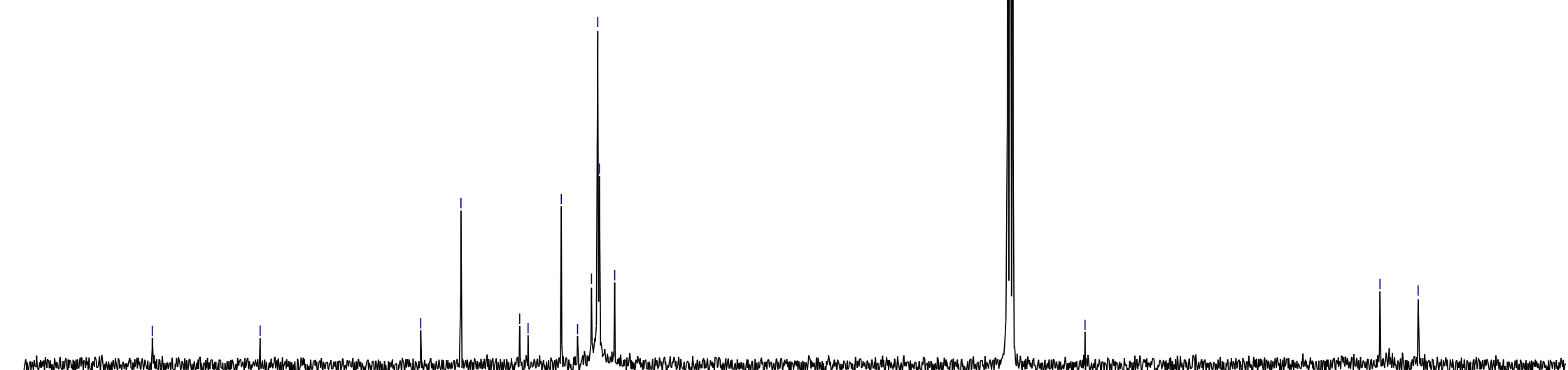




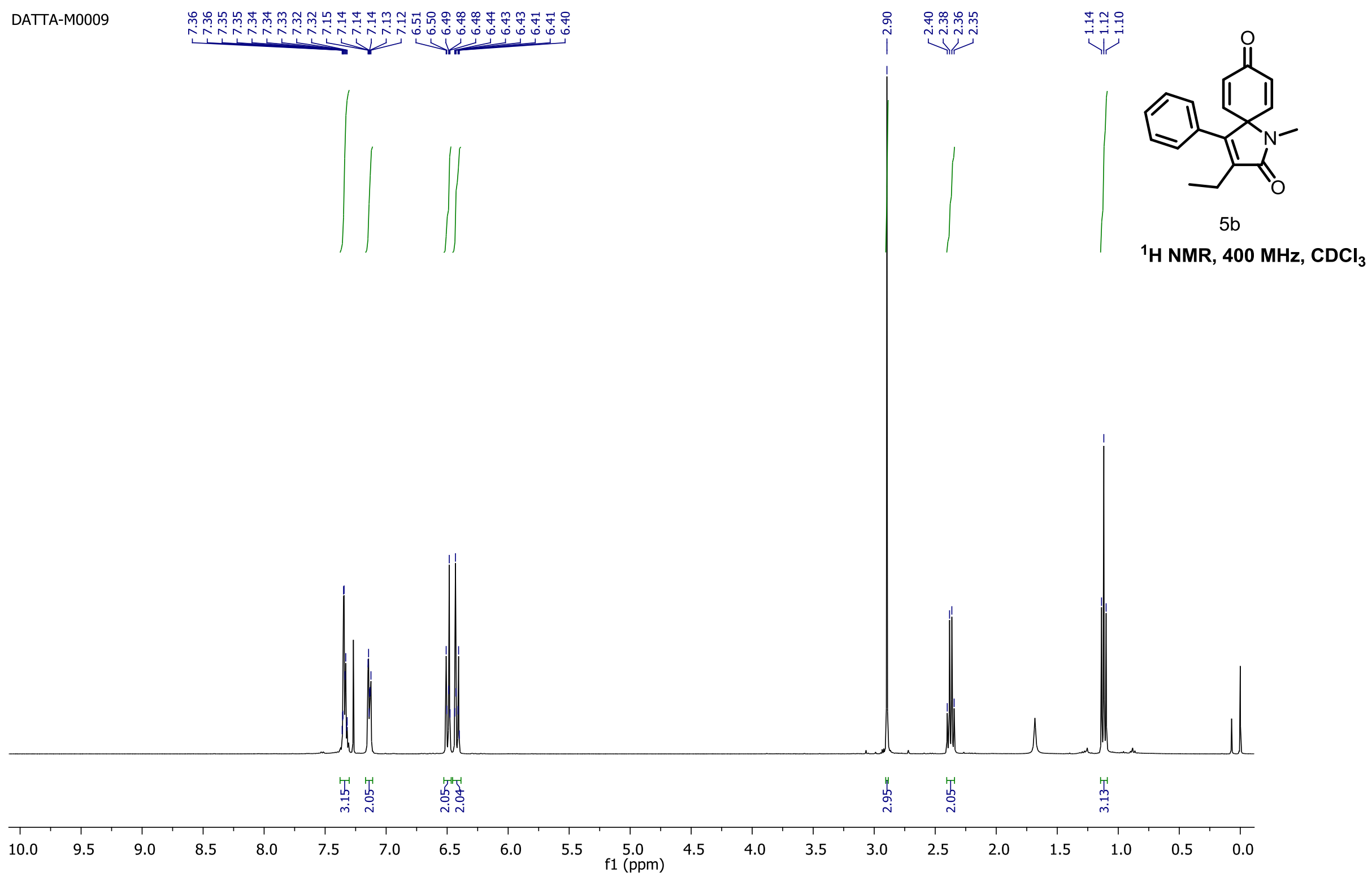




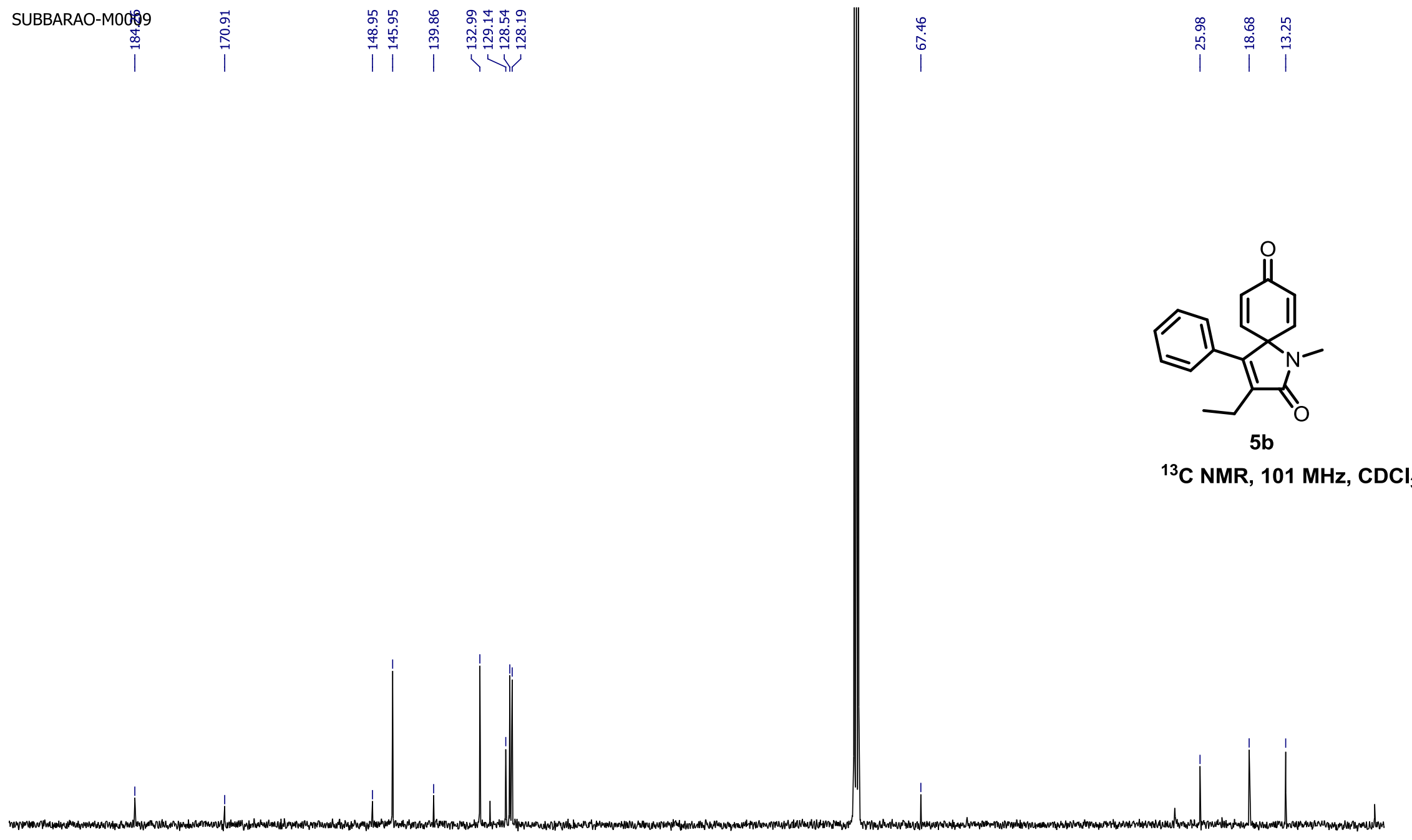

\begin{tabular}{|c|c|c|c|c|c|c|c|c|c|c|c|c|c|c|c|c|c|c|c|c|}
\hline 200 & 190 & 180 & 170 & 160 & 150 & 140 & 130 & 120 & 110 & $\begin{array}{c}100 \\
\mathrm{f} 1(\mathrm{ppm})\end{array}$ & 90 & 80 & 70 & 60 & 50 & 40 & 30 & 20 & 10 & 0 \\
\hline
\end{tabular}




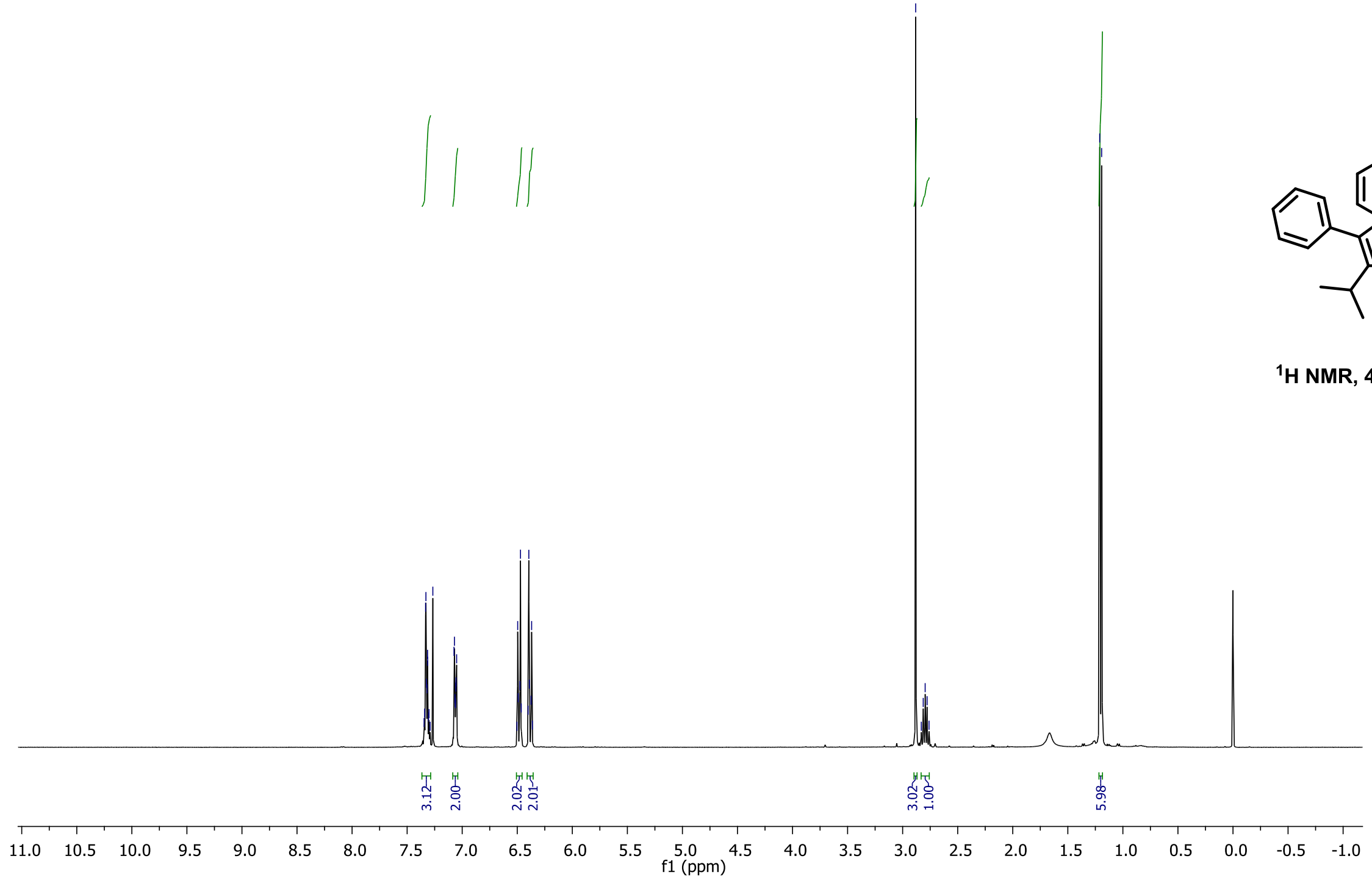



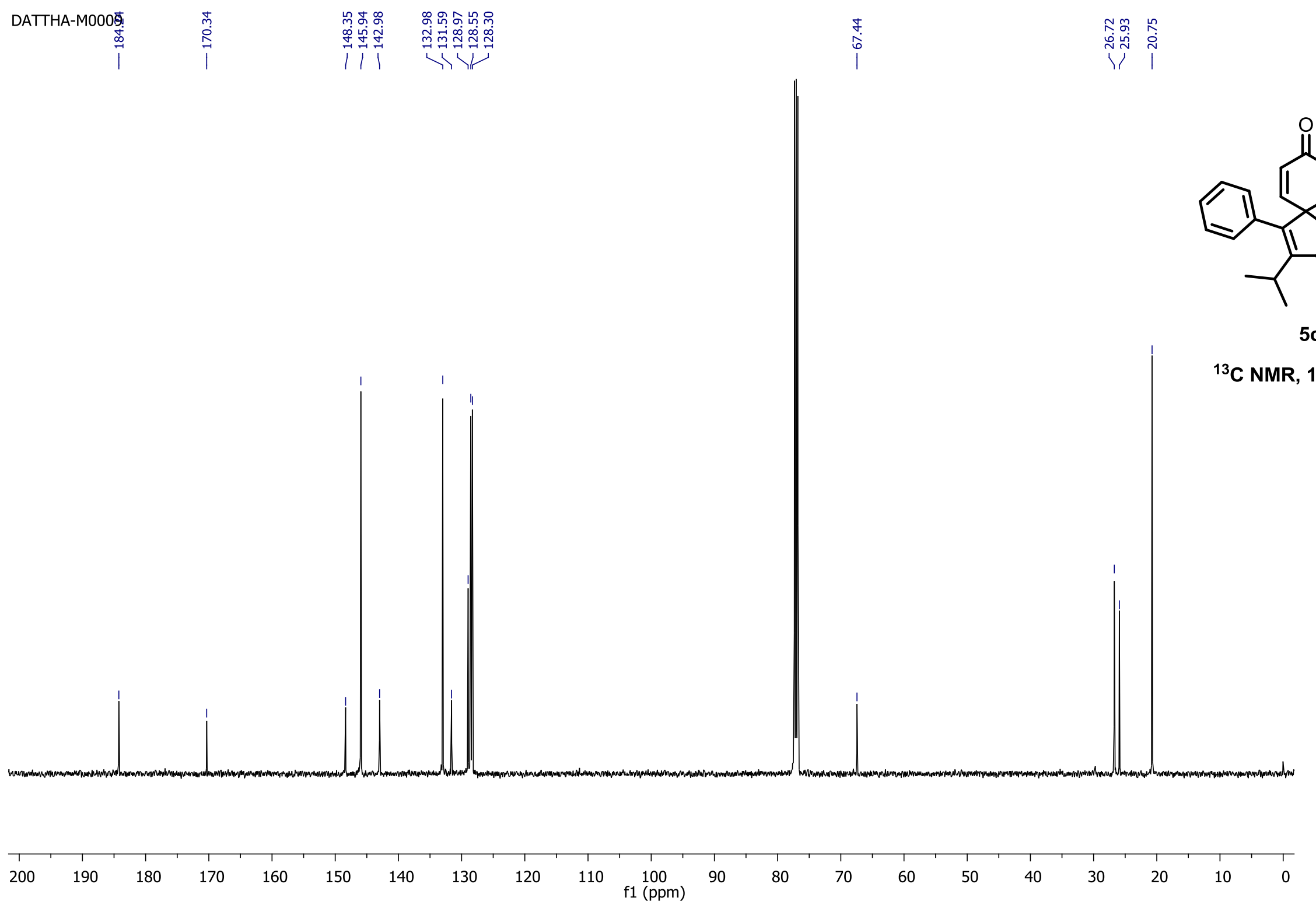


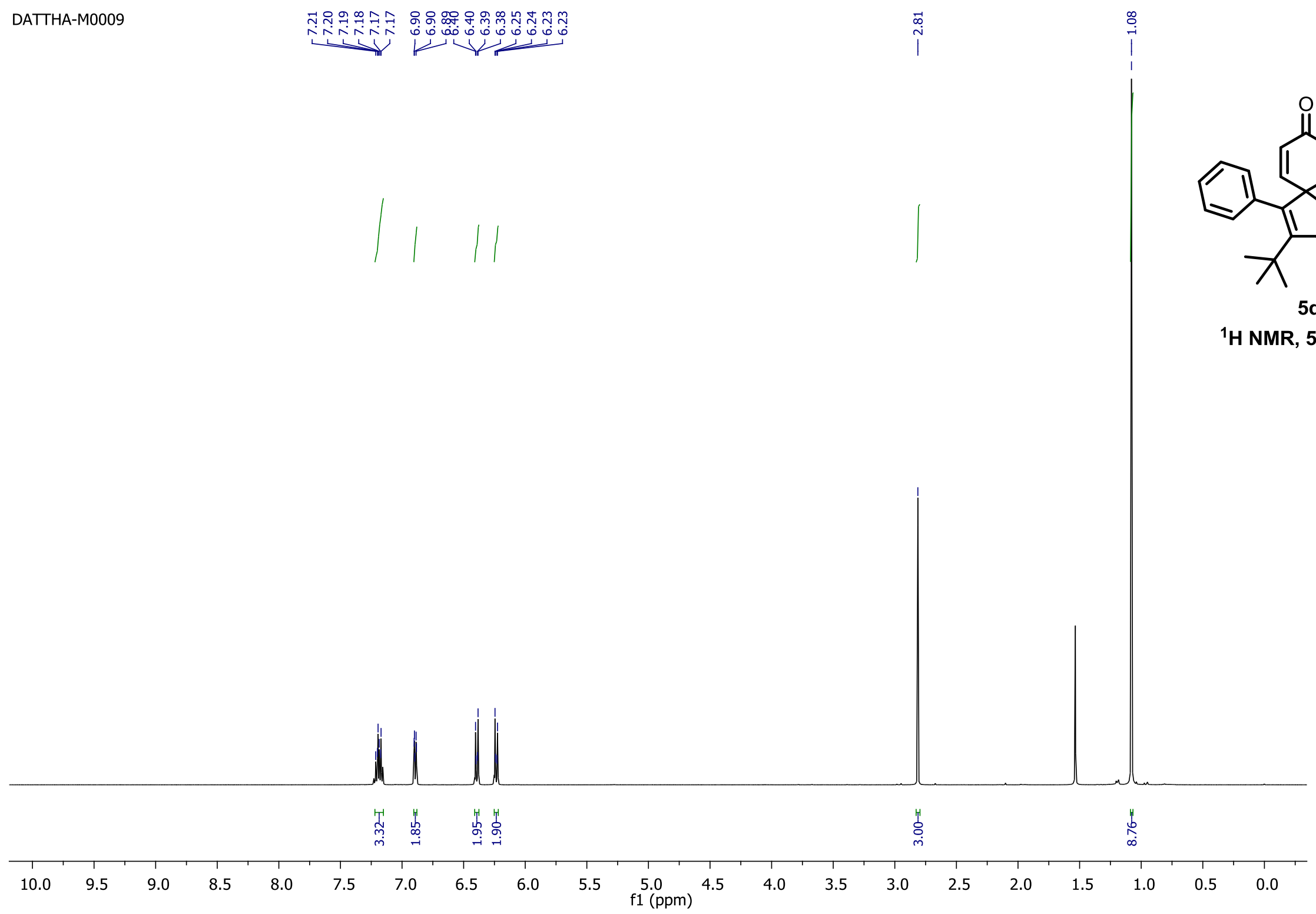




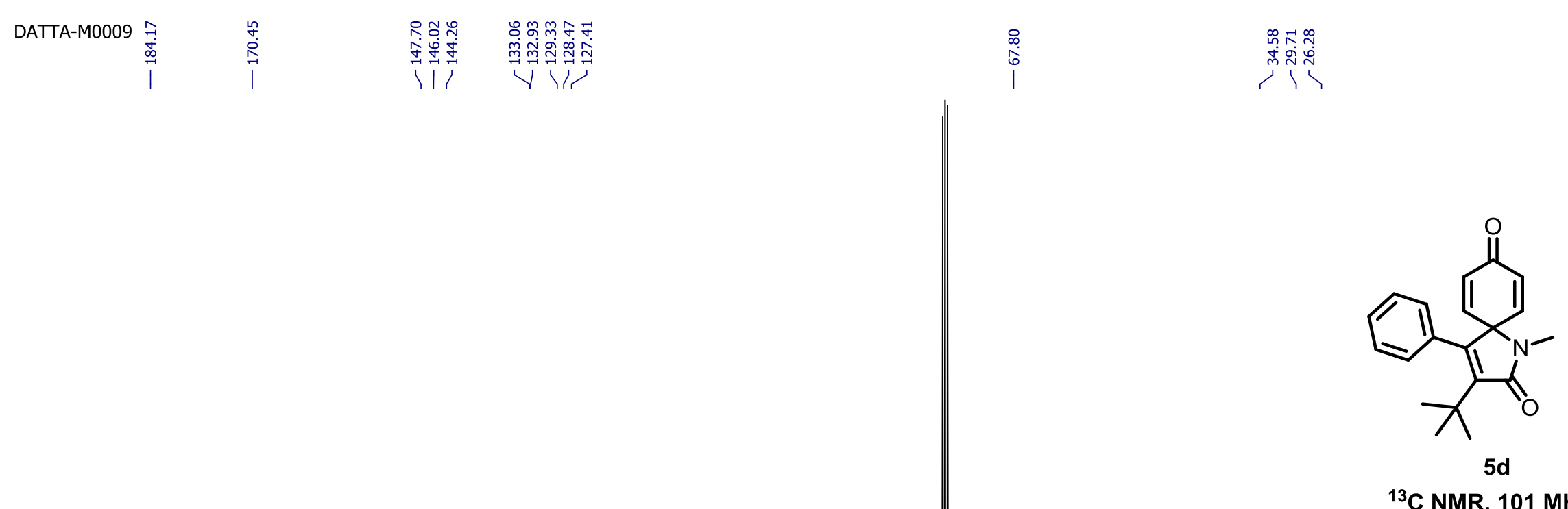

${ }^{13} \mathrm{C}$ NMR, $101 \mathrm{MHz}, \mathrm{CDCl}_{3}$
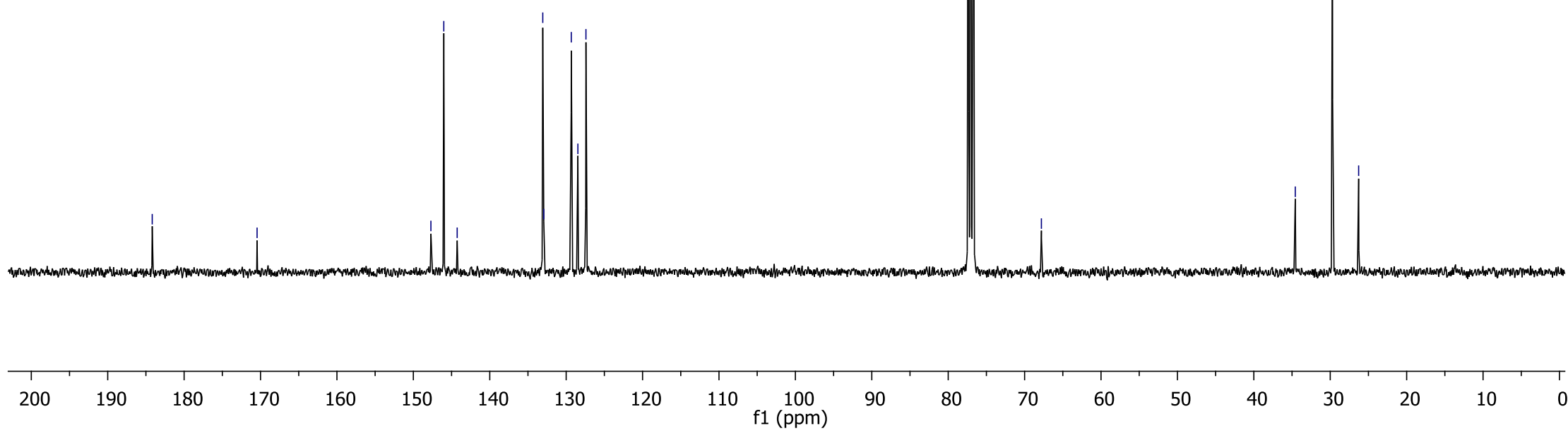


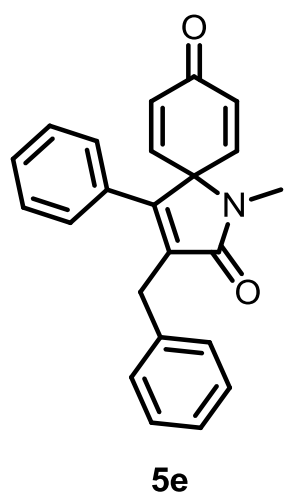

${ }^{1} \mathrm{H}$ NMR, $400 \mathrm{MHz}, \mathrm{CDCl}_{3}$

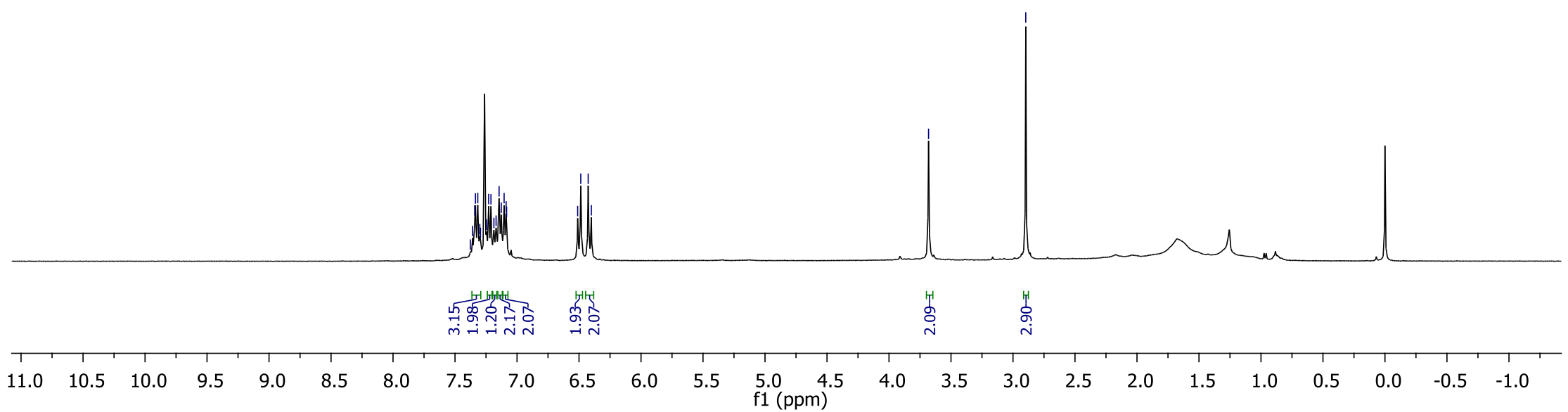




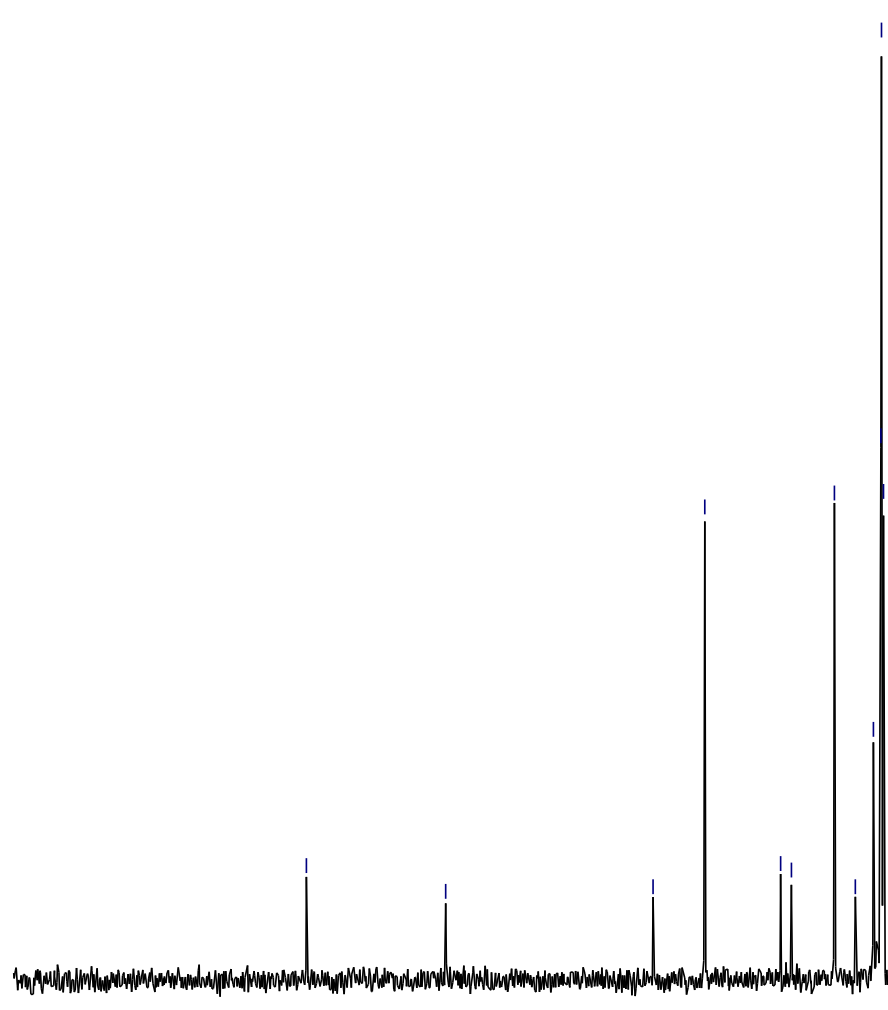

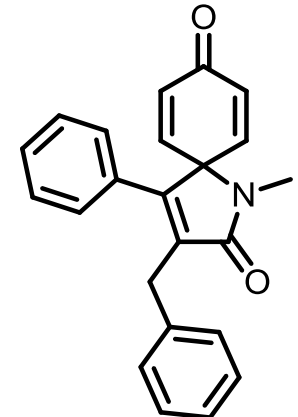

$5 e$

${ }^{13} \mathrm{C} \mathrm{NMR}, 101 \mathrm{MHz}, \mathrm{CDCl}_{3}$ 


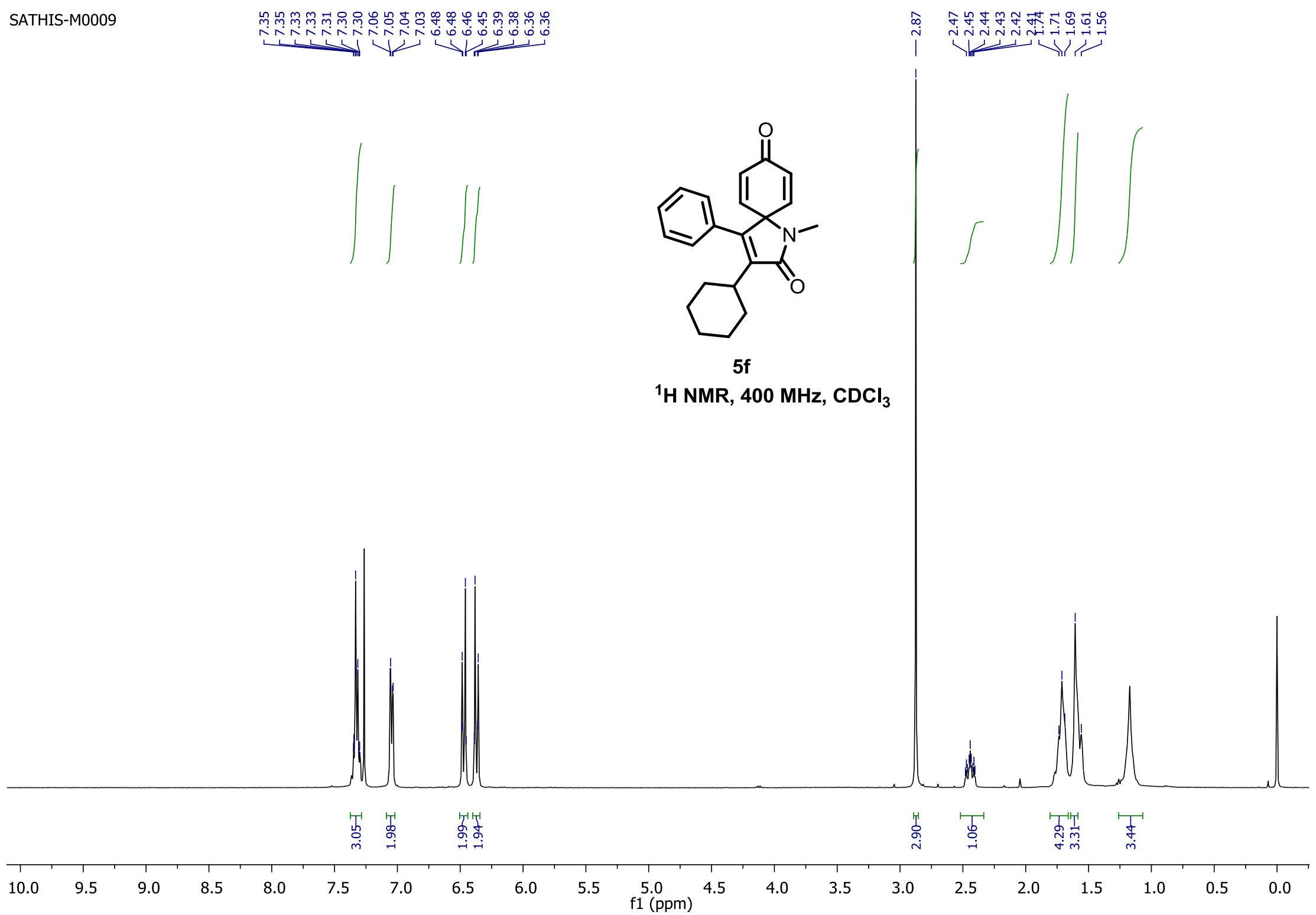




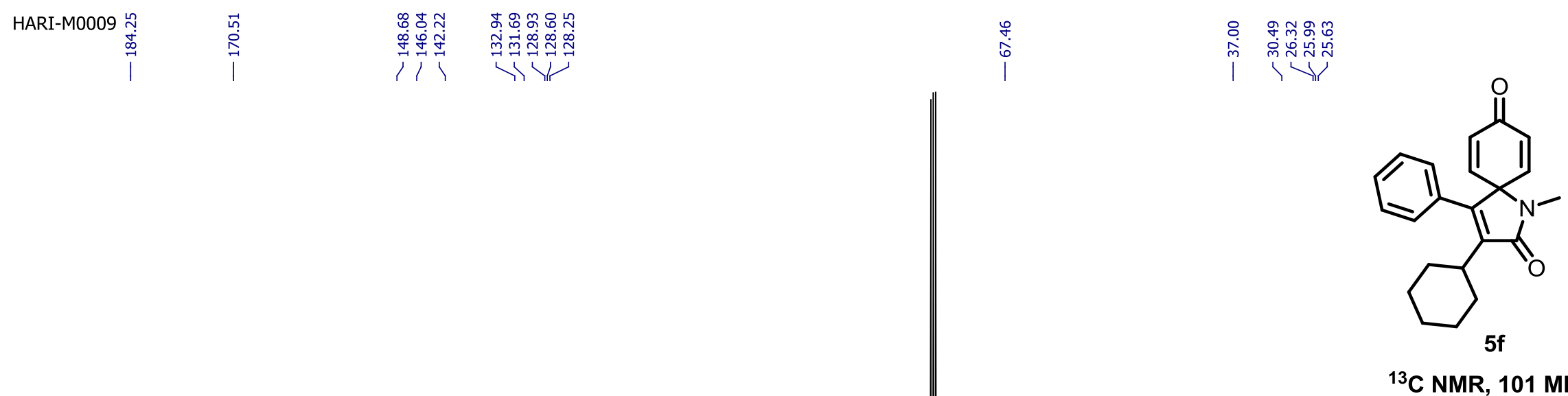

${ }^{13} \mathrm{C} \mathrm{NMR}, 101 \mathrm{MHz}, \mathrm{CDCl}_{3}$
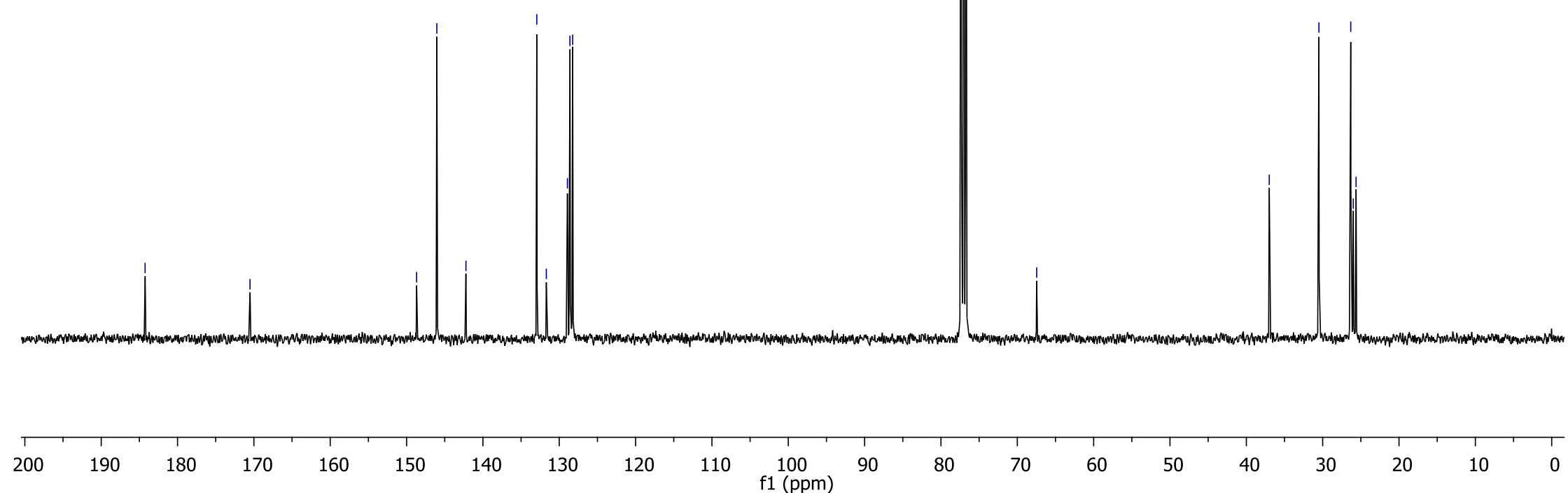
ROSAN M0009

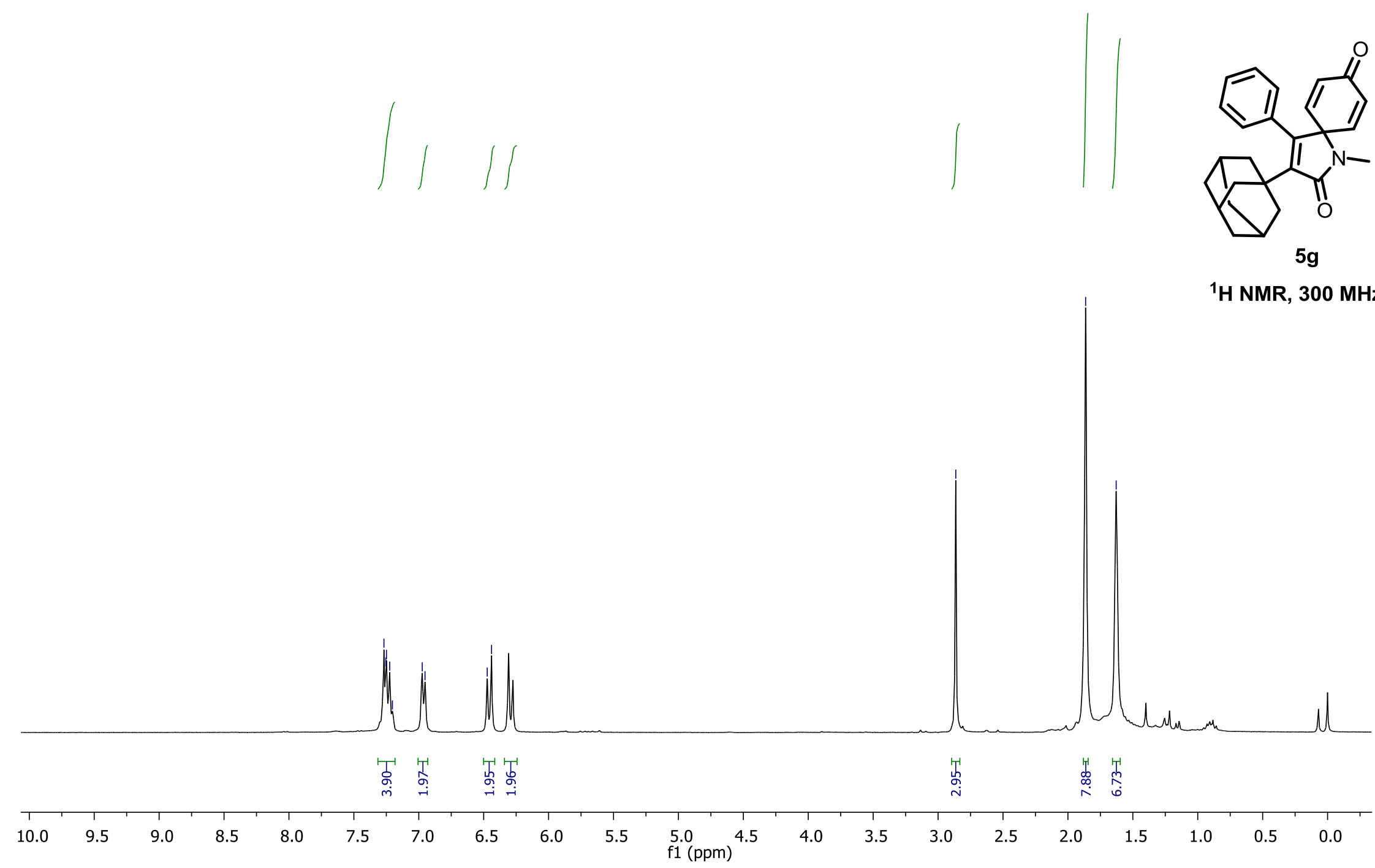


SRINIVAS-M000委

:

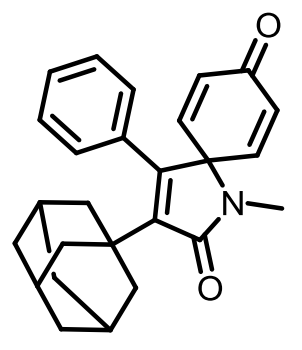

$5 g$

${ }^{13} \mathrm{C}$ NMR, $101 \mathrm{MHz}, \mathrm{CDCl}_{3}$

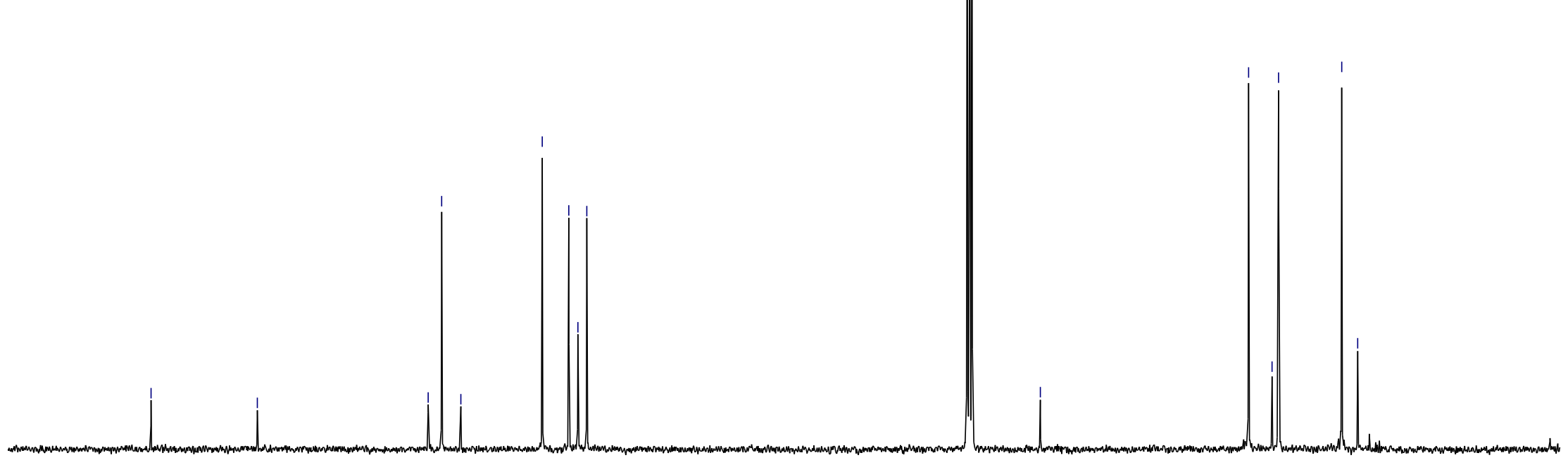




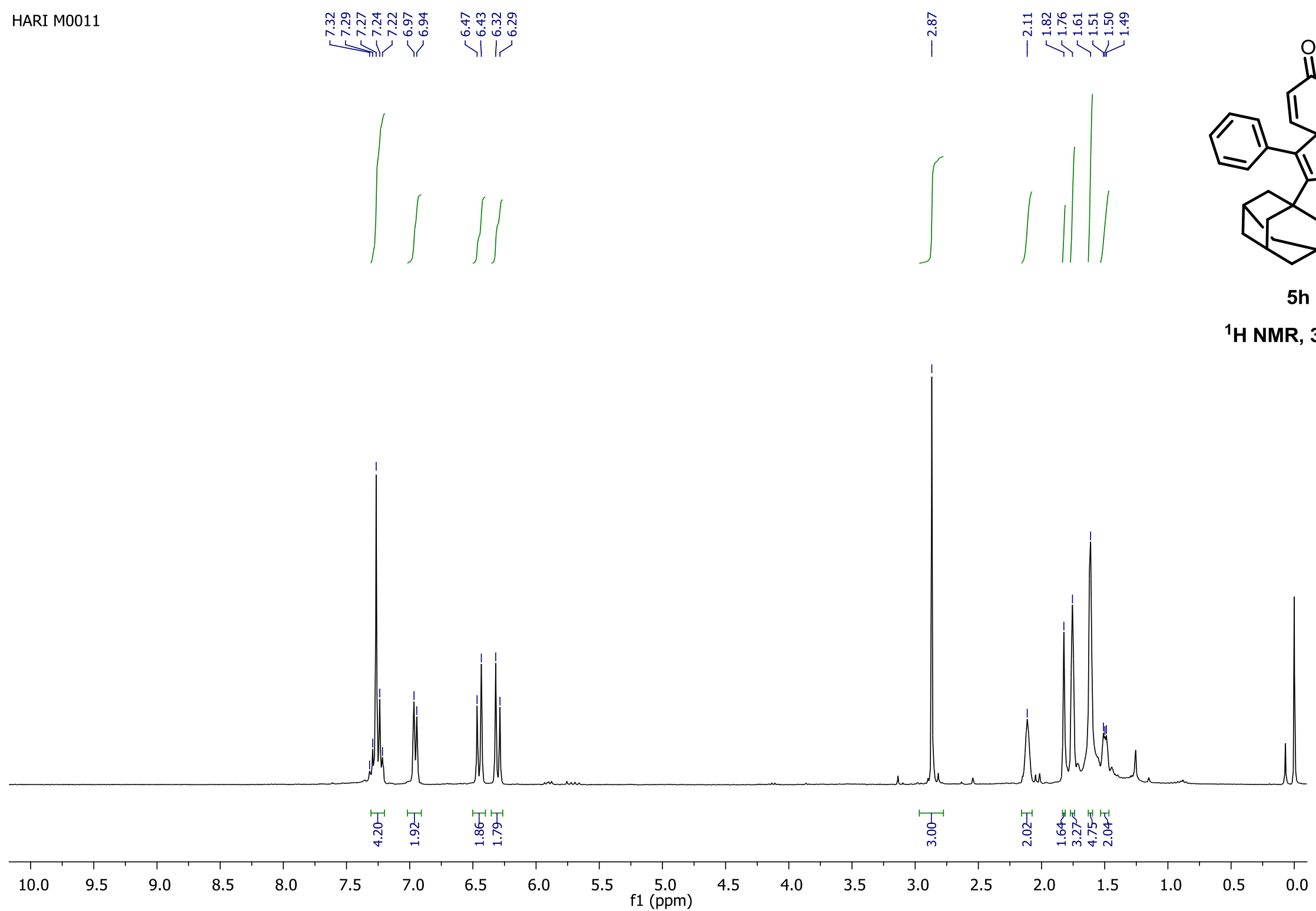



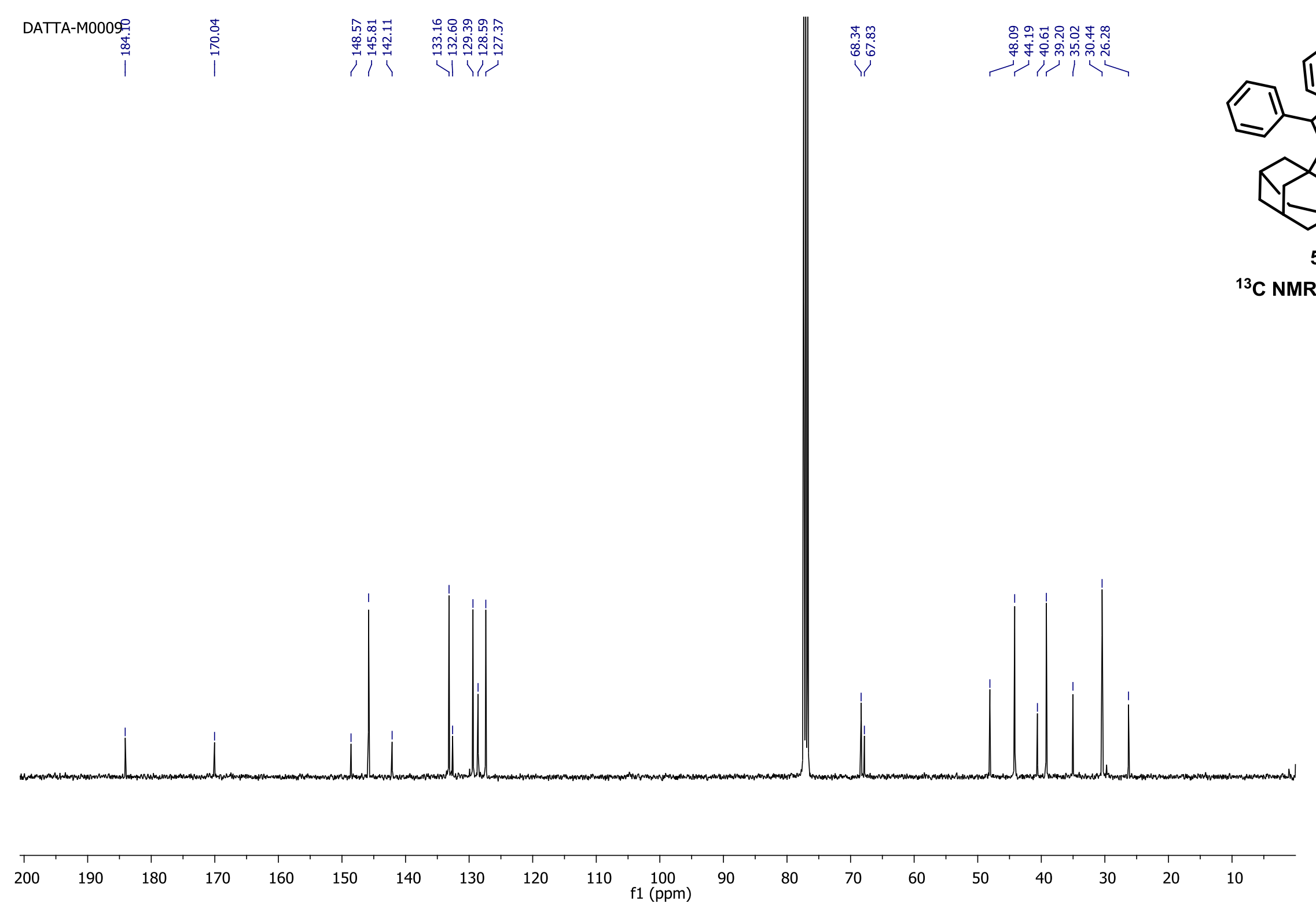


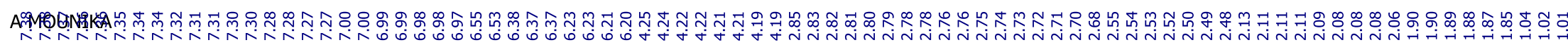

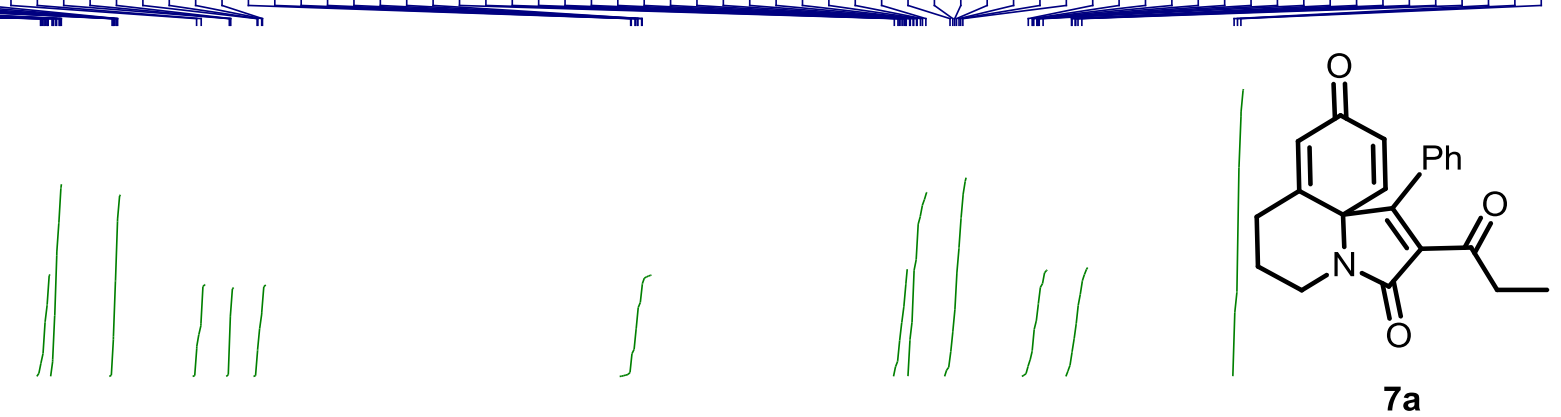

${ }^{1} \mathrm{H}$ NMR, $400 \mathrm{MHz}, \mathrm{CDCl}_{3}$

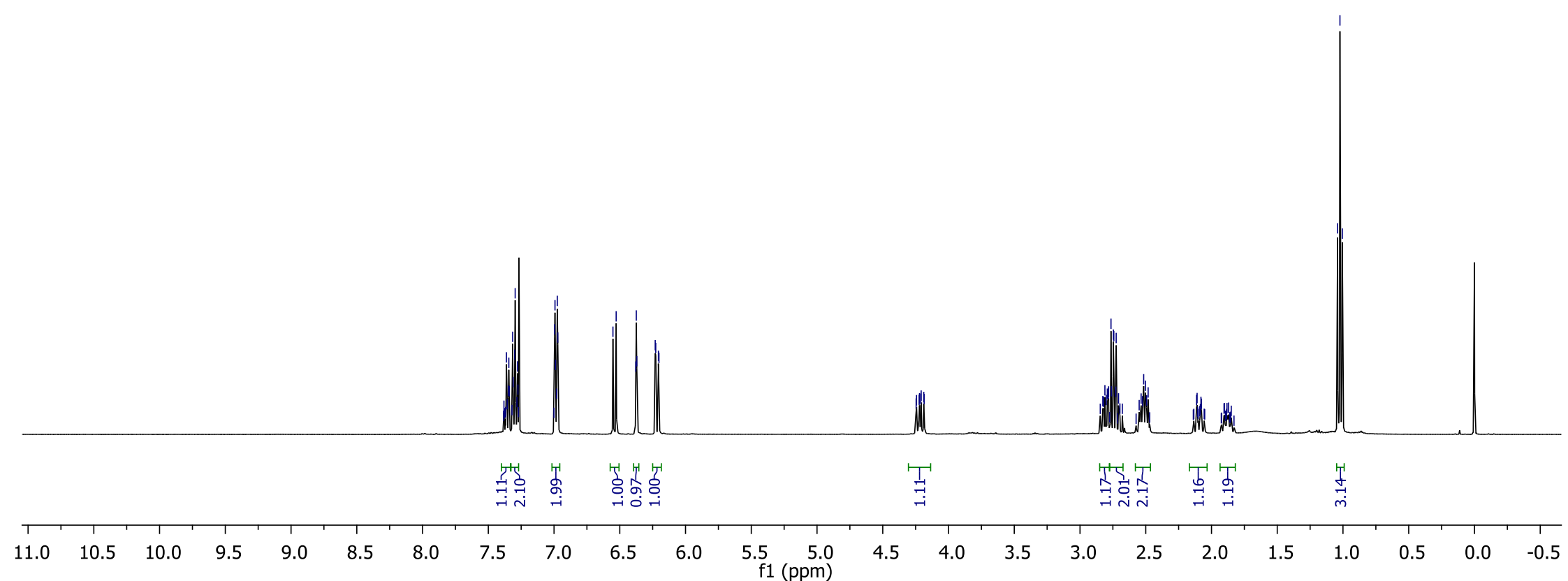



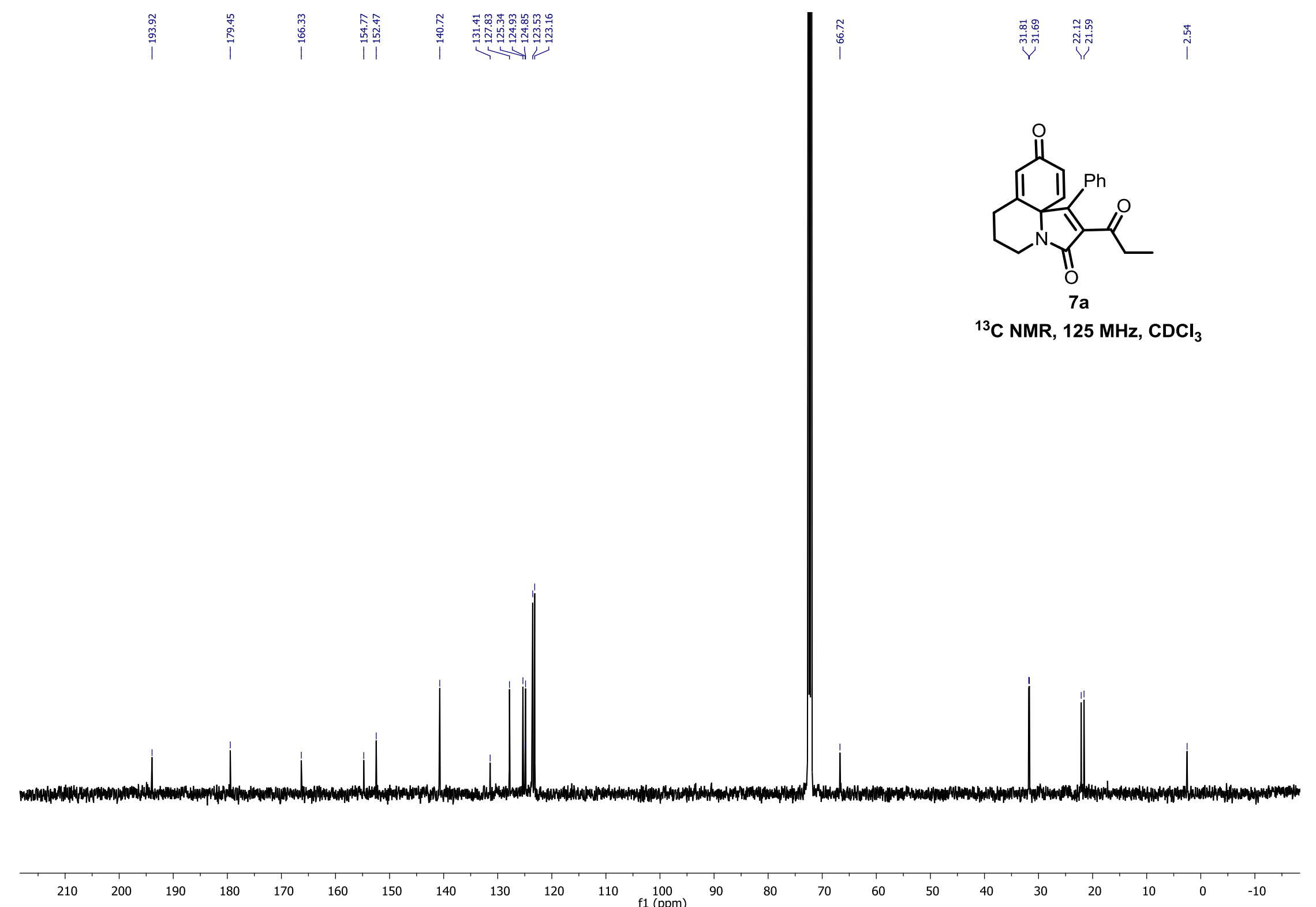


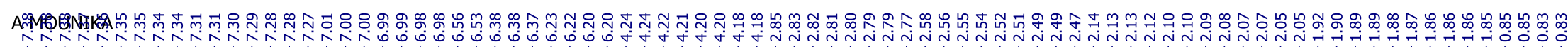

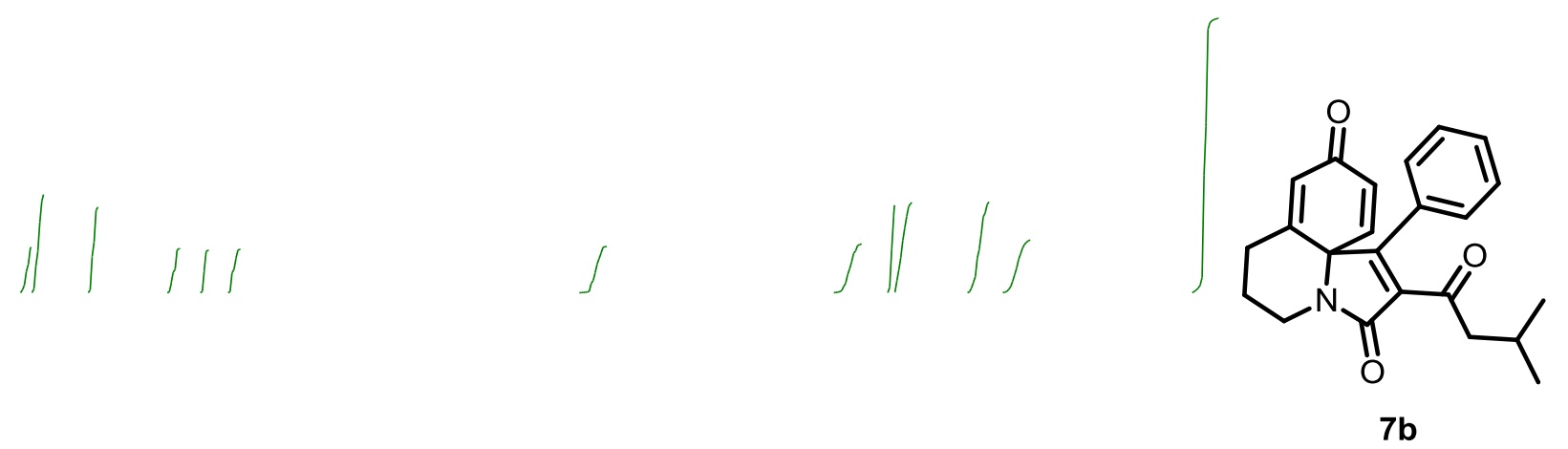

${ }^{1} \mathrm{H}$ NMR, $500 \mathrm{MHz}, \mathrm{CDCl}_{3}$

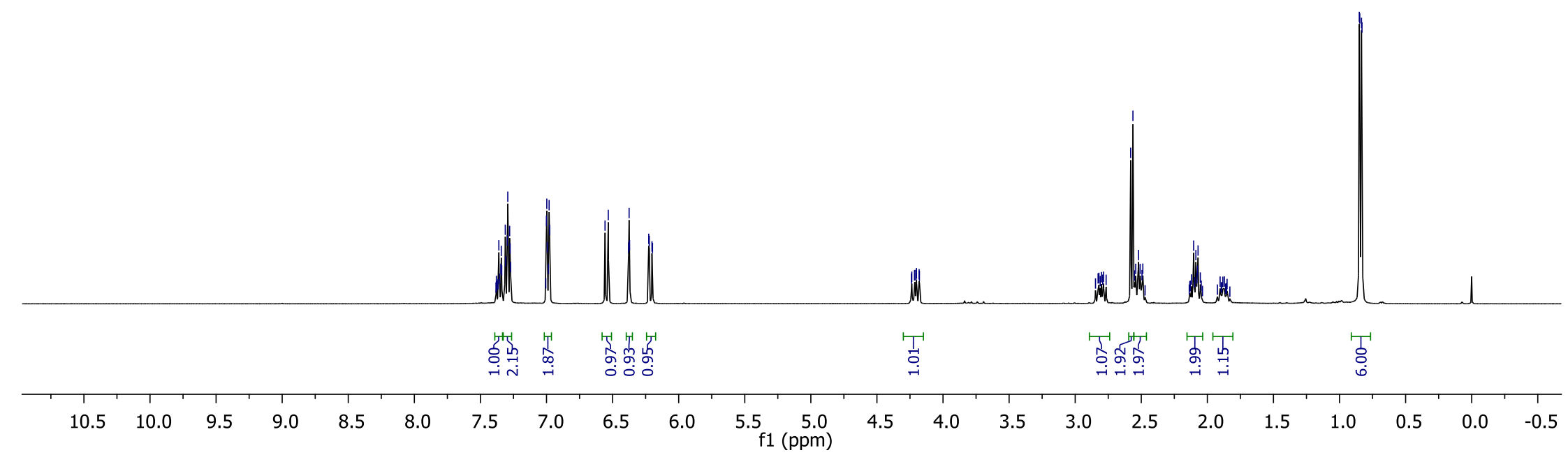




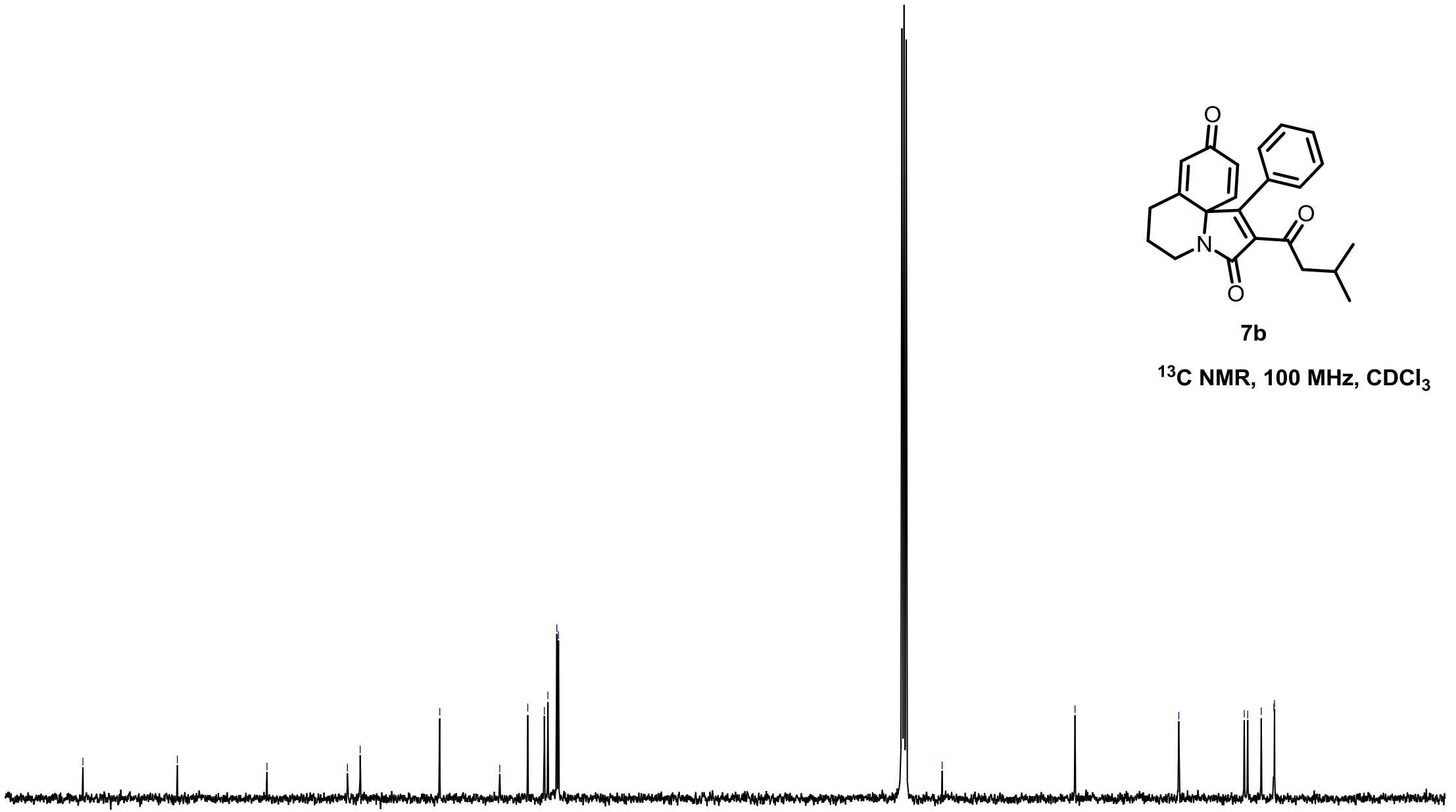




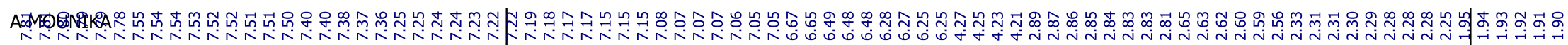

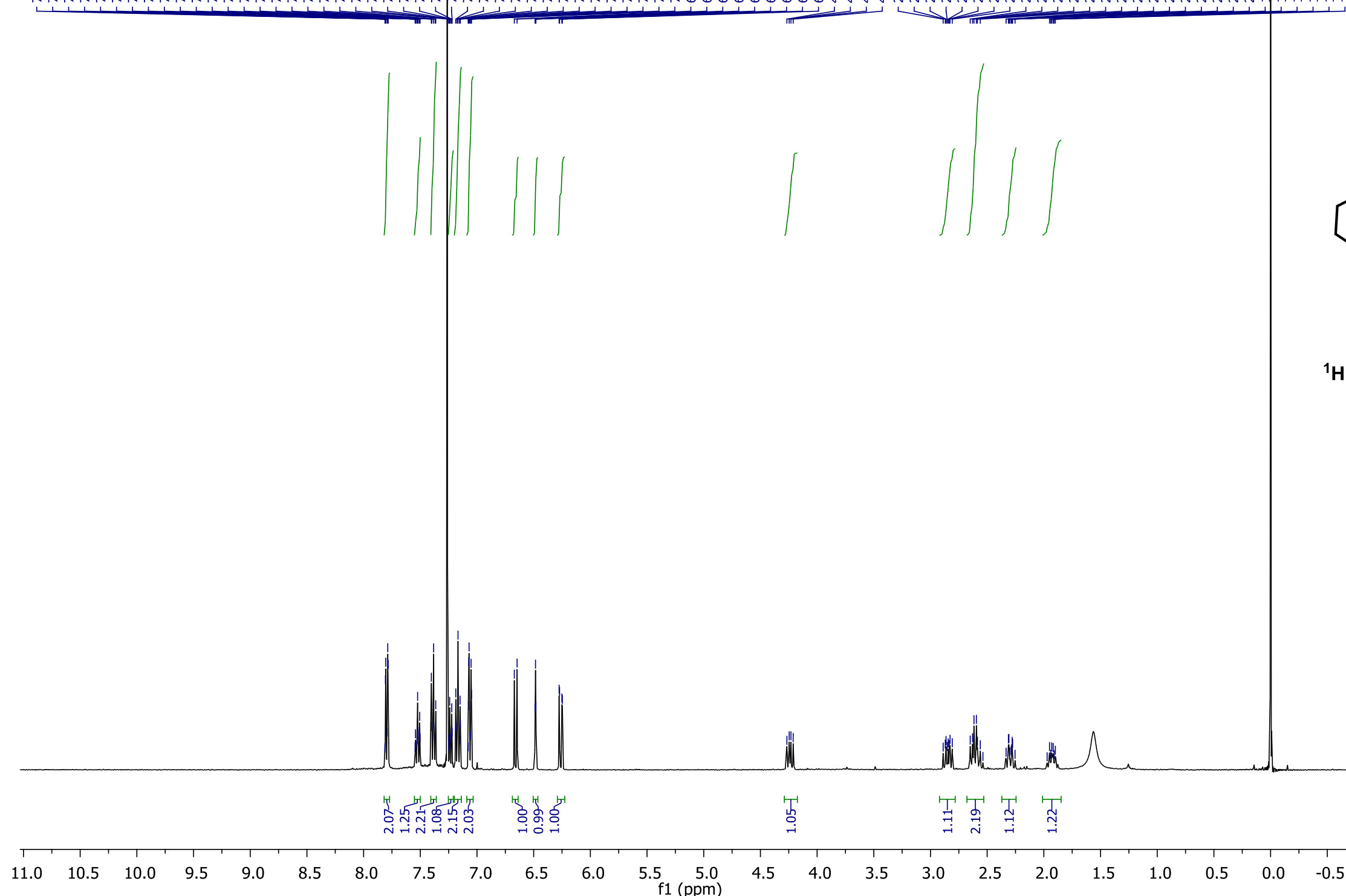




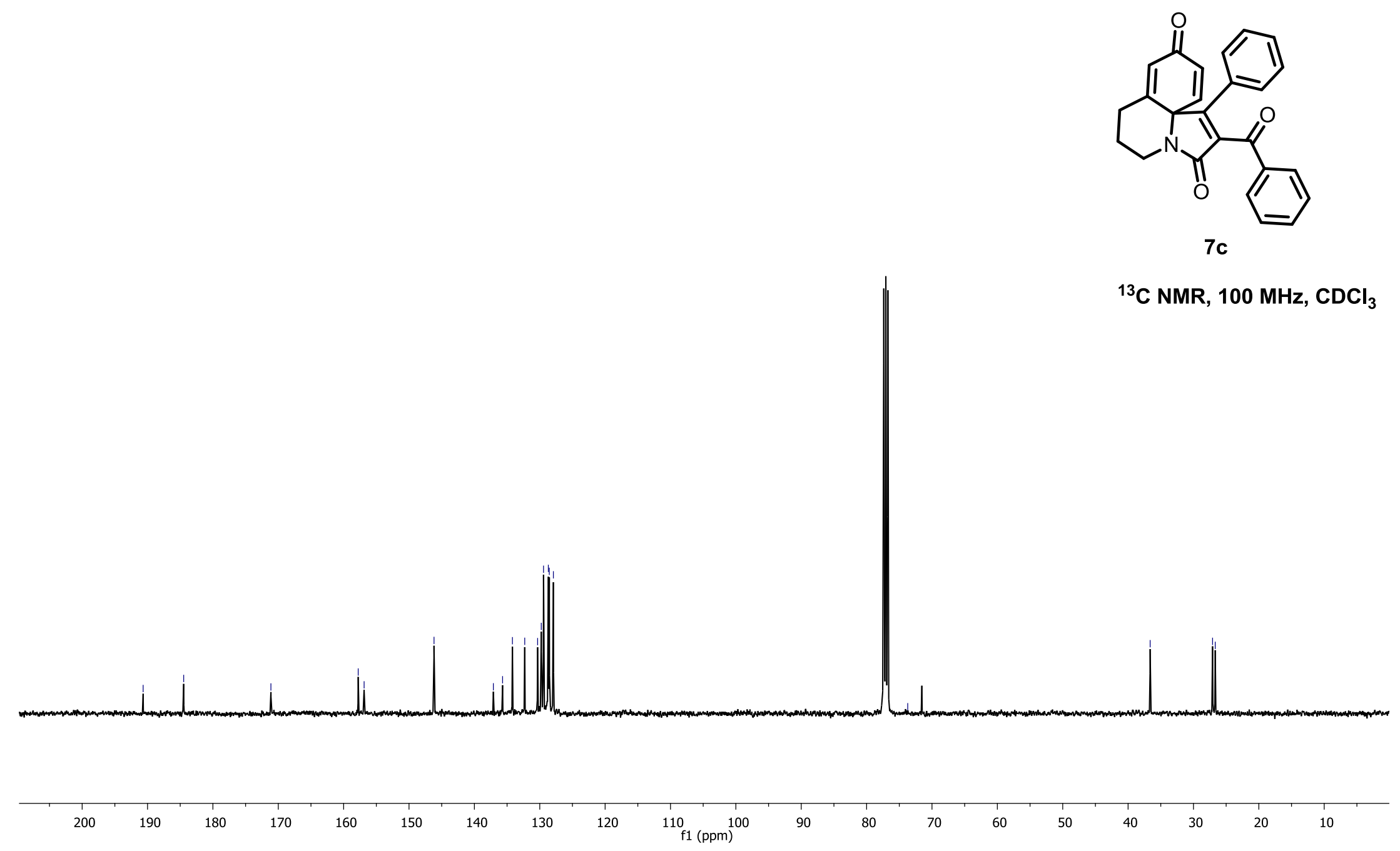




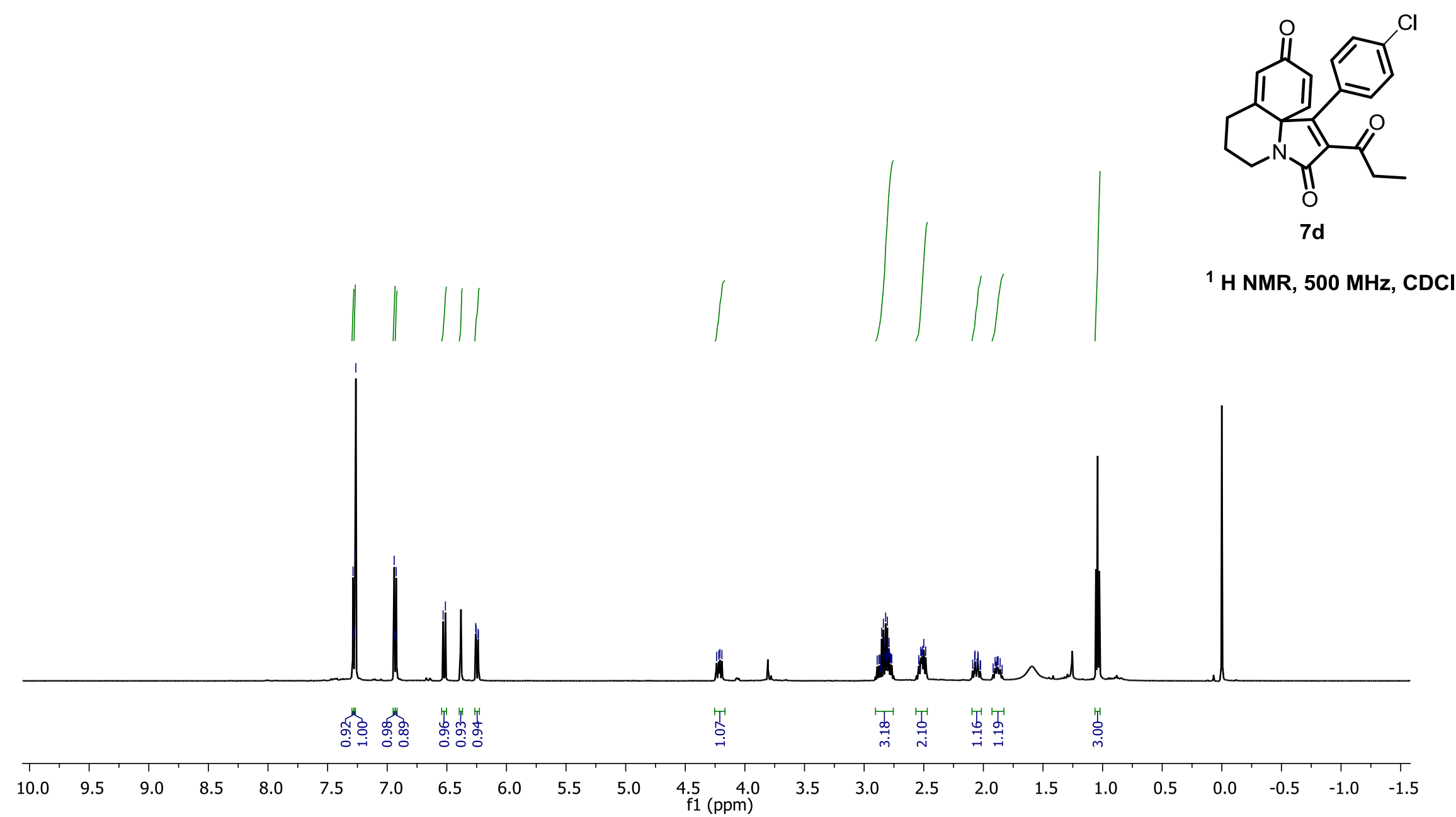




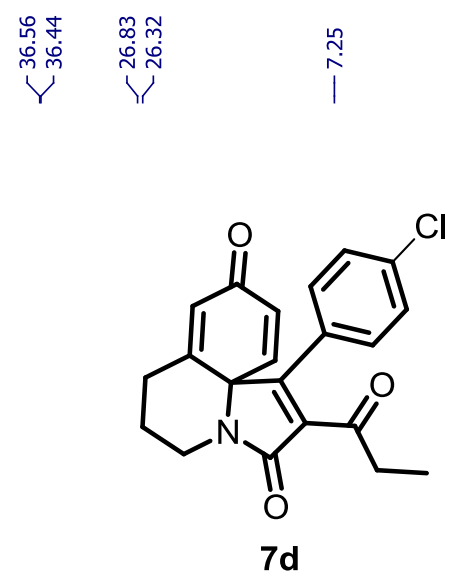

${ }^{13} \mathrm{C} \mathrm{NMR}, 100 \mathrm{MHz}, \mathrm{CDCl}_{3}$
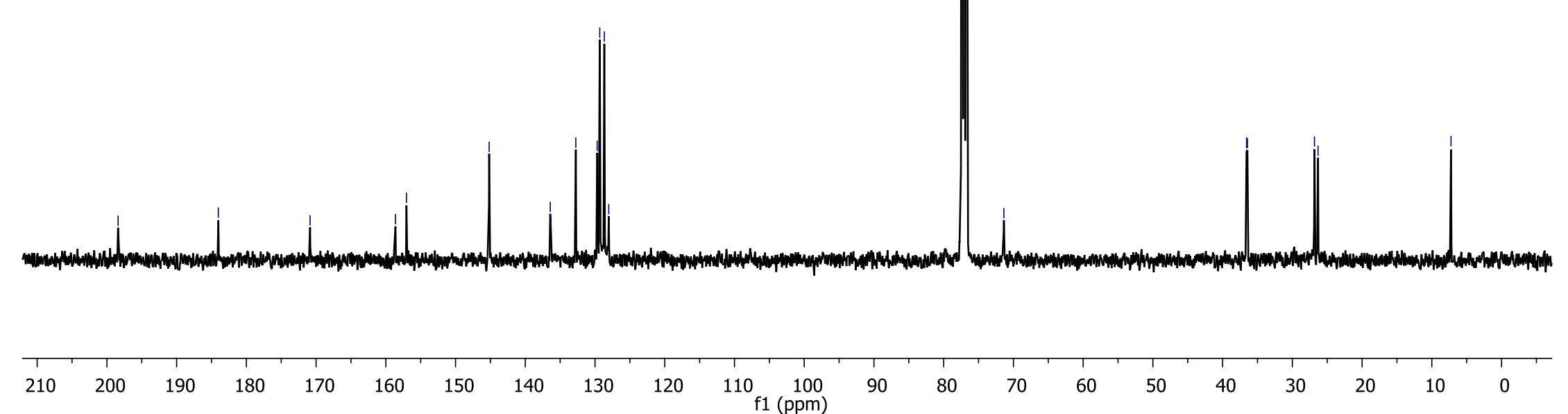


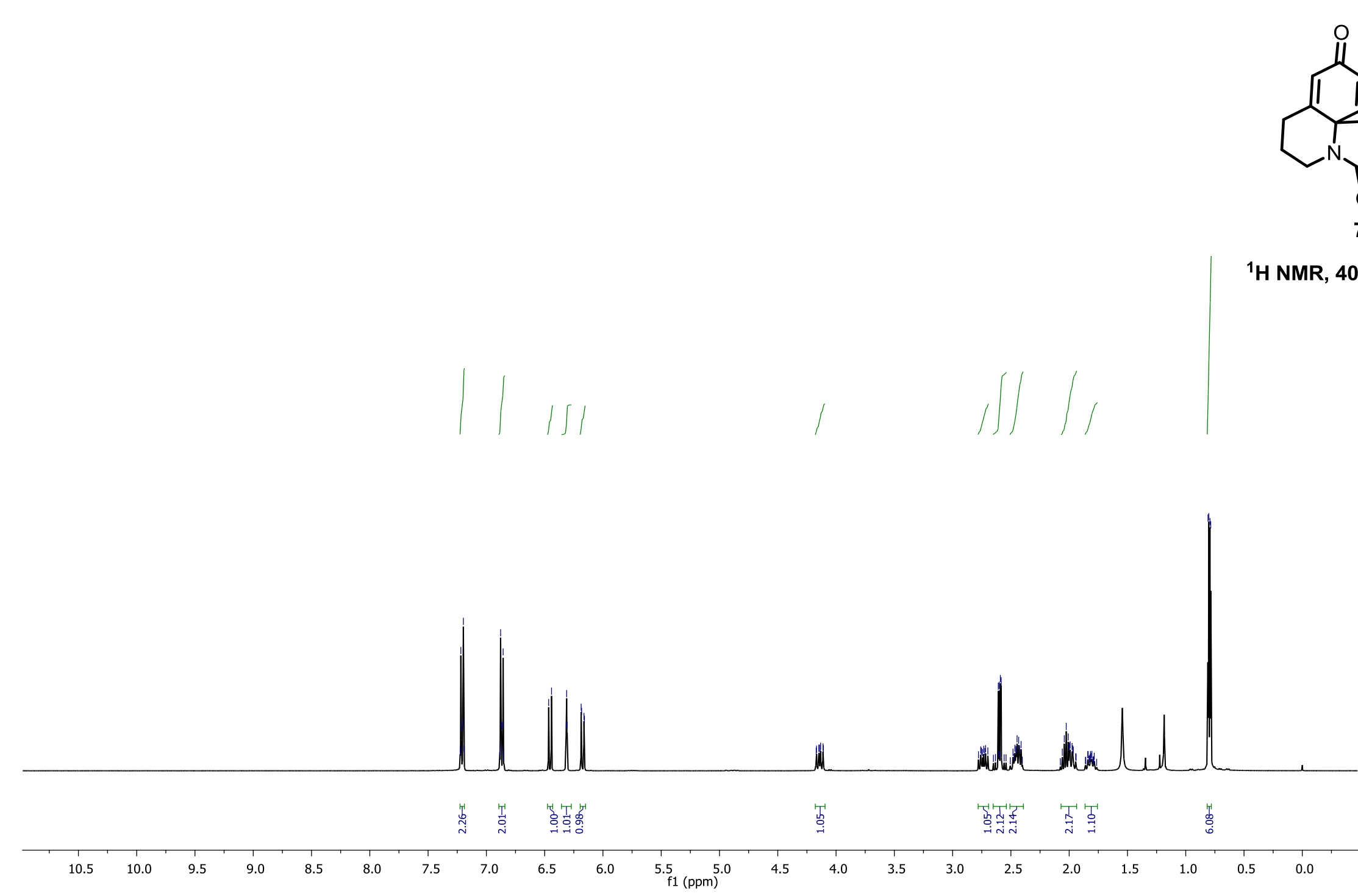



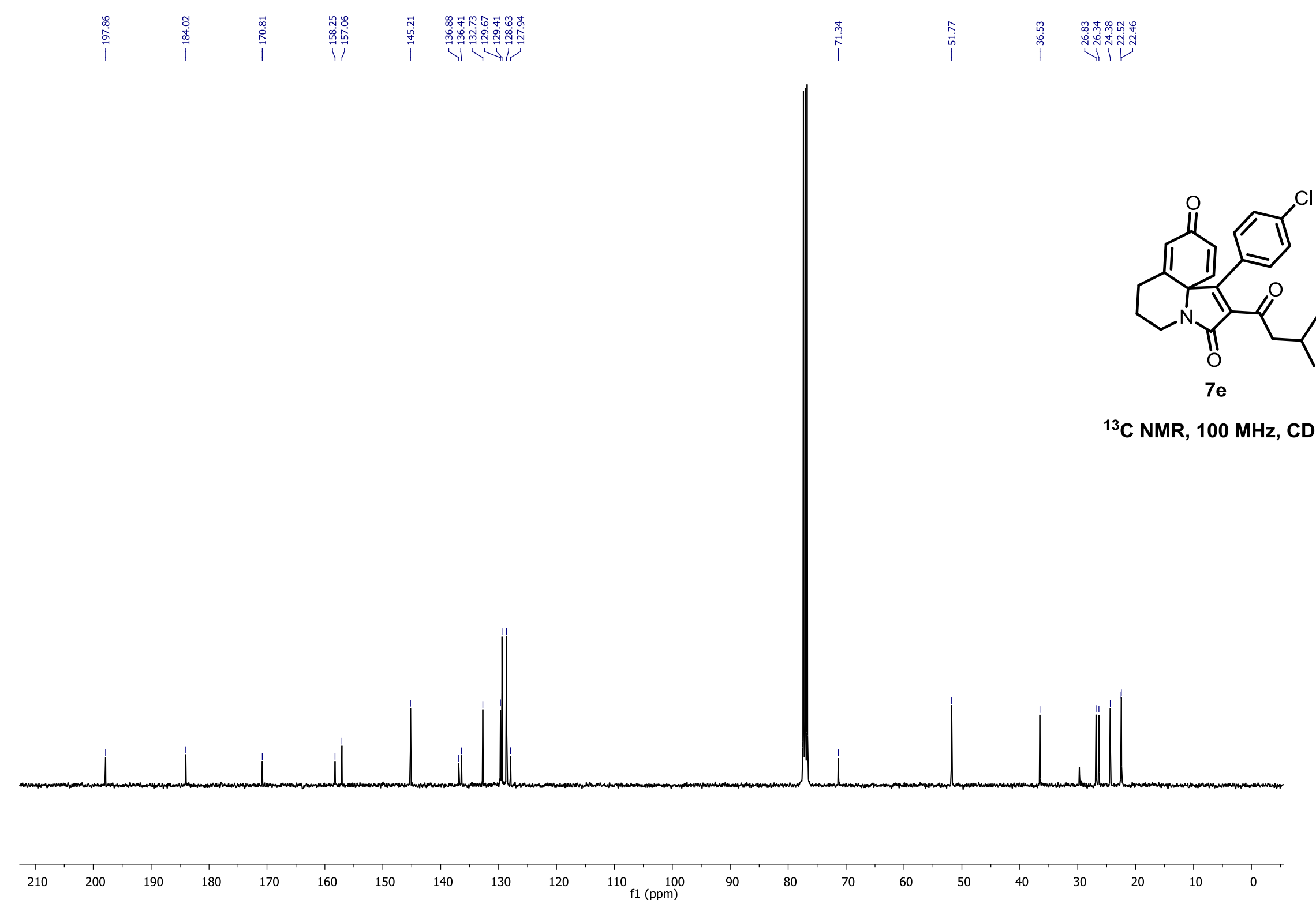


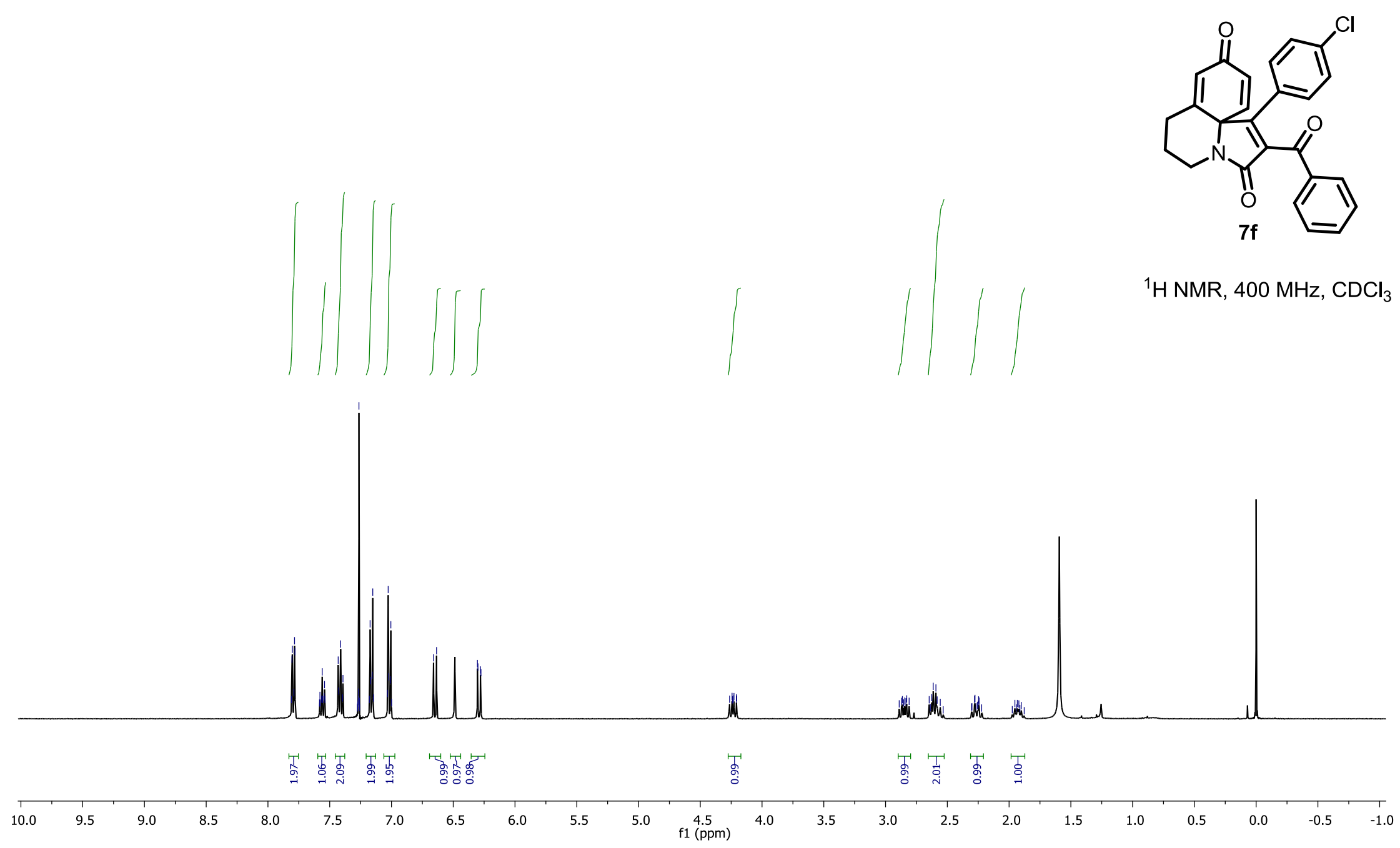




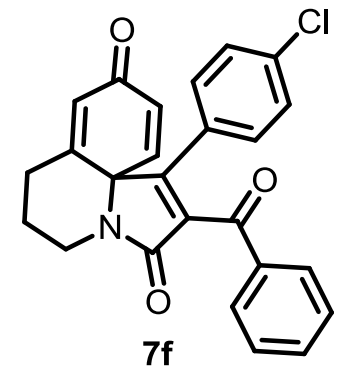

${ }^{13} \mathrm{C} \mathrm{NMR,}, 100 \mathrm{MHz}, \mathrm{CDCl}_{3}$
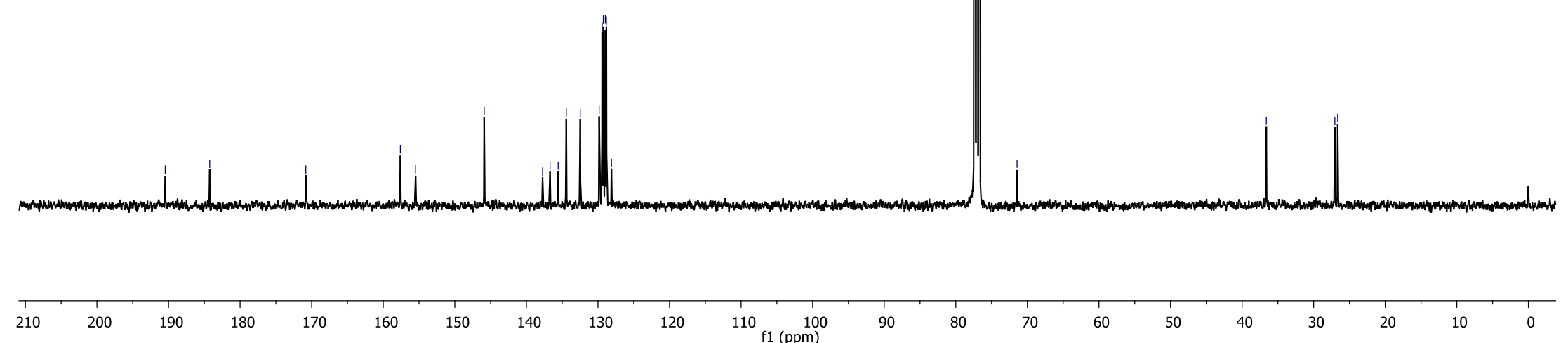


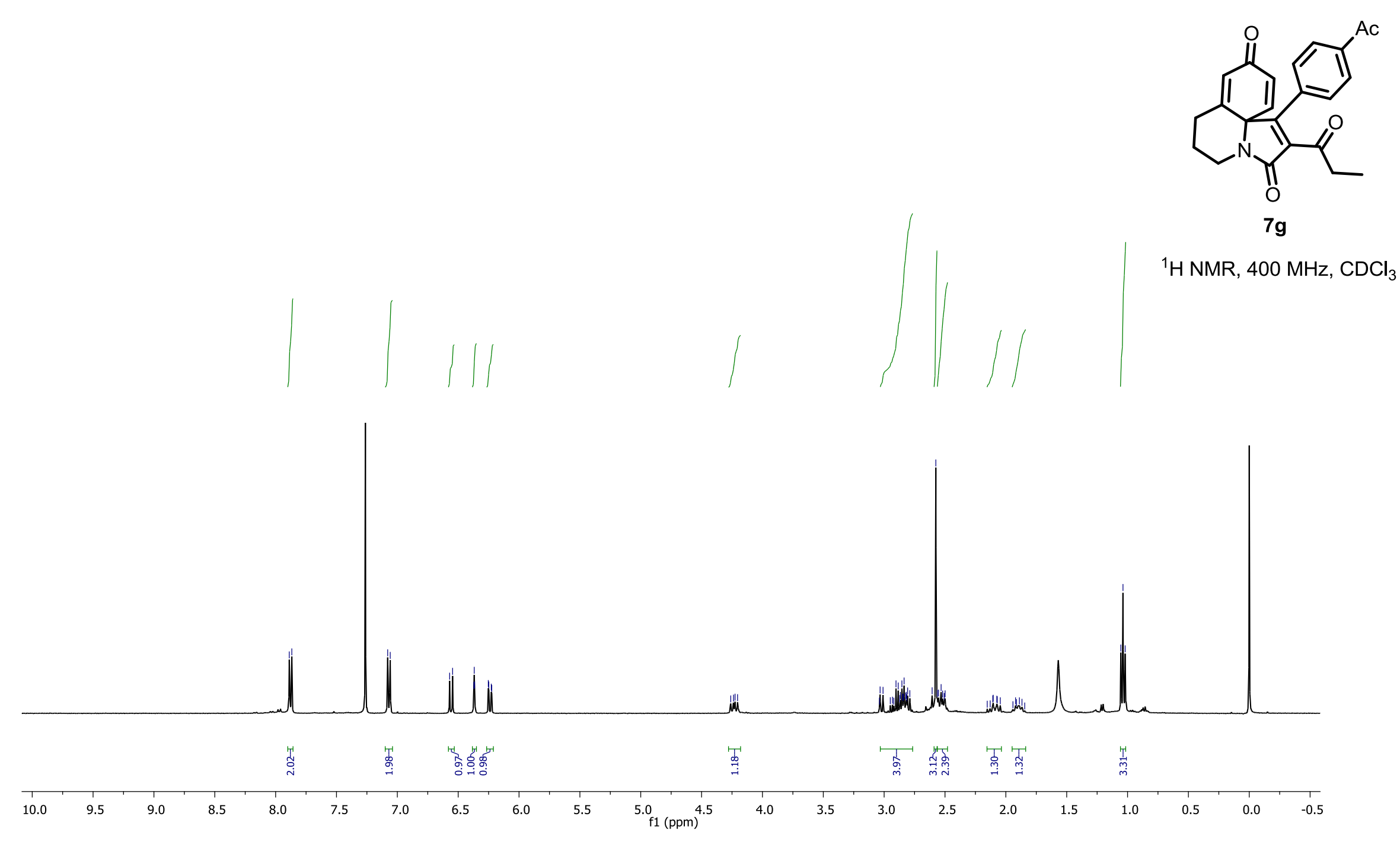




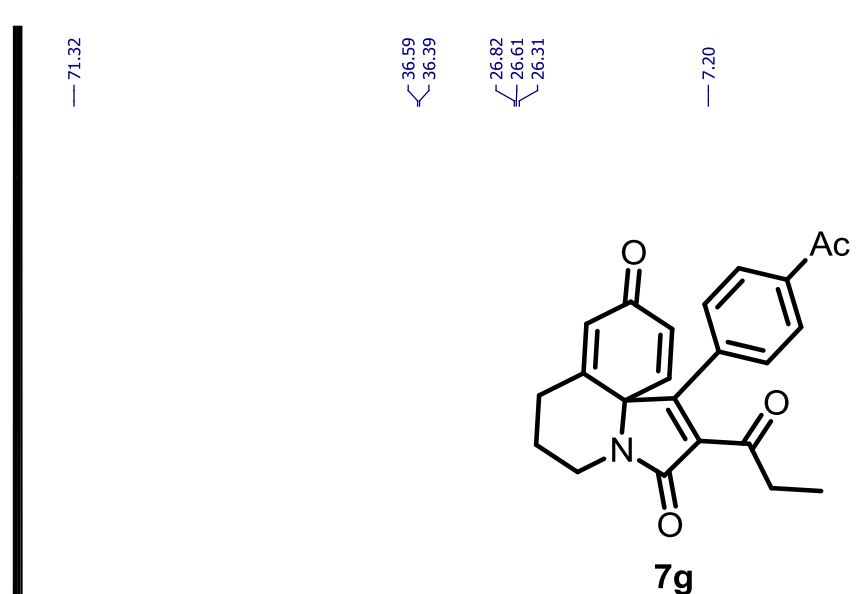

${ }^{13} \mathrm{C} \mathrm{NMR,} 100 \mathrm{MHz}^{\mathrm{CDCl}}{ }_{3}$

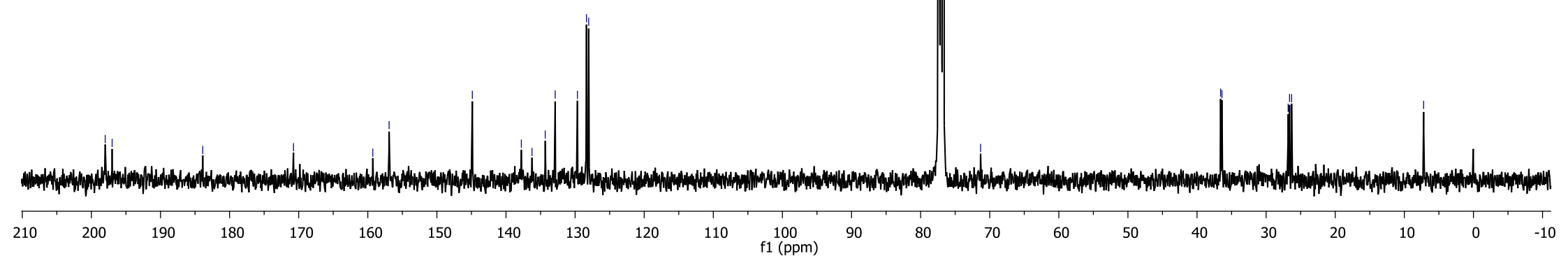




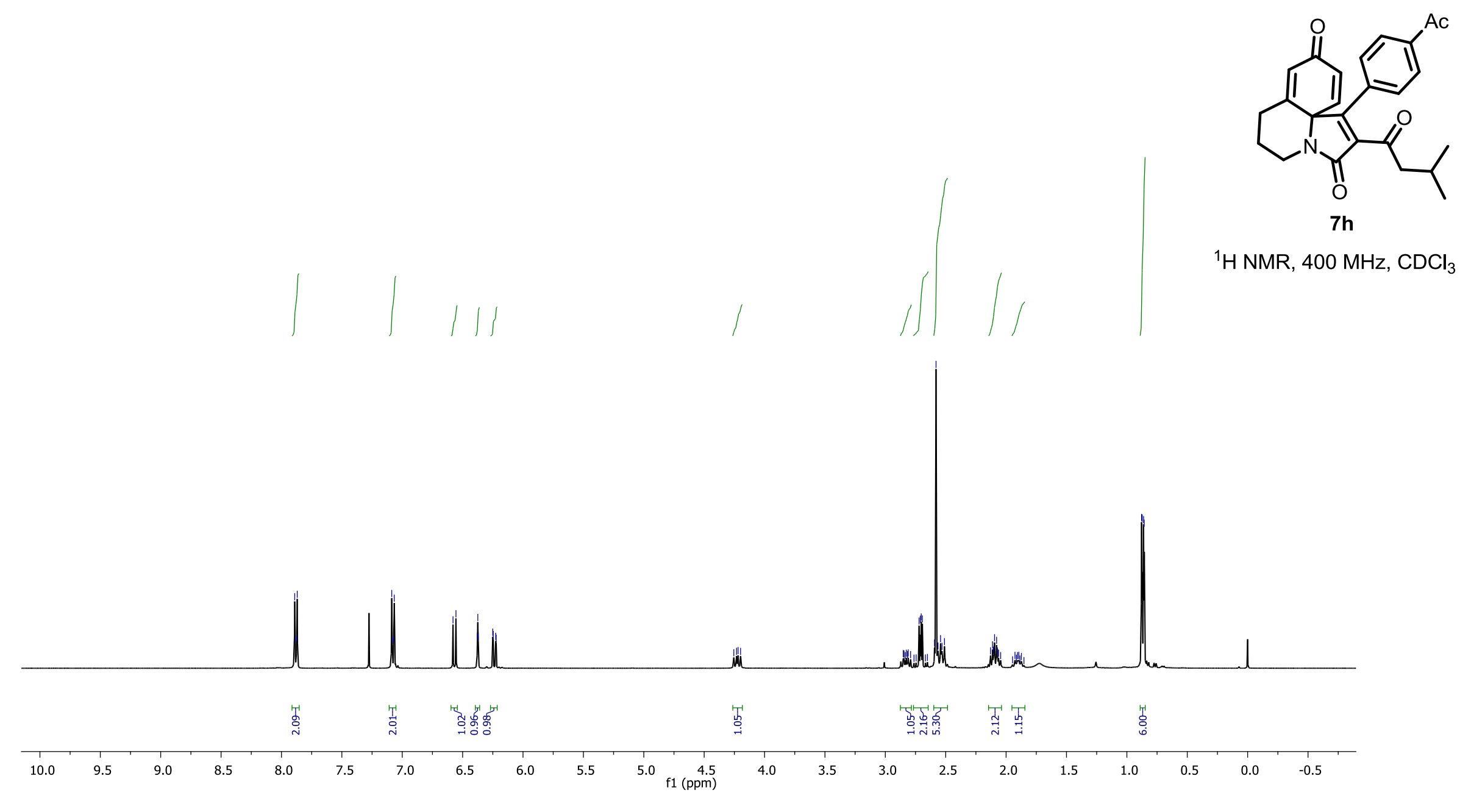




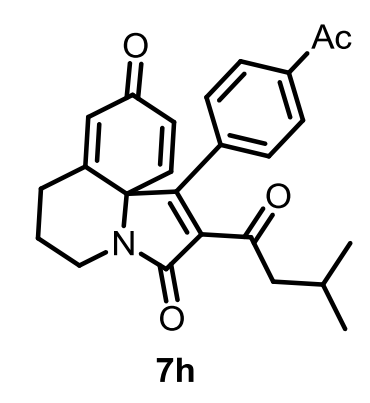

${ }^{13} \mathrm{C}$ NMR, $100 \mathrm{MHz}, \mathrm{CDCl}_{3}$
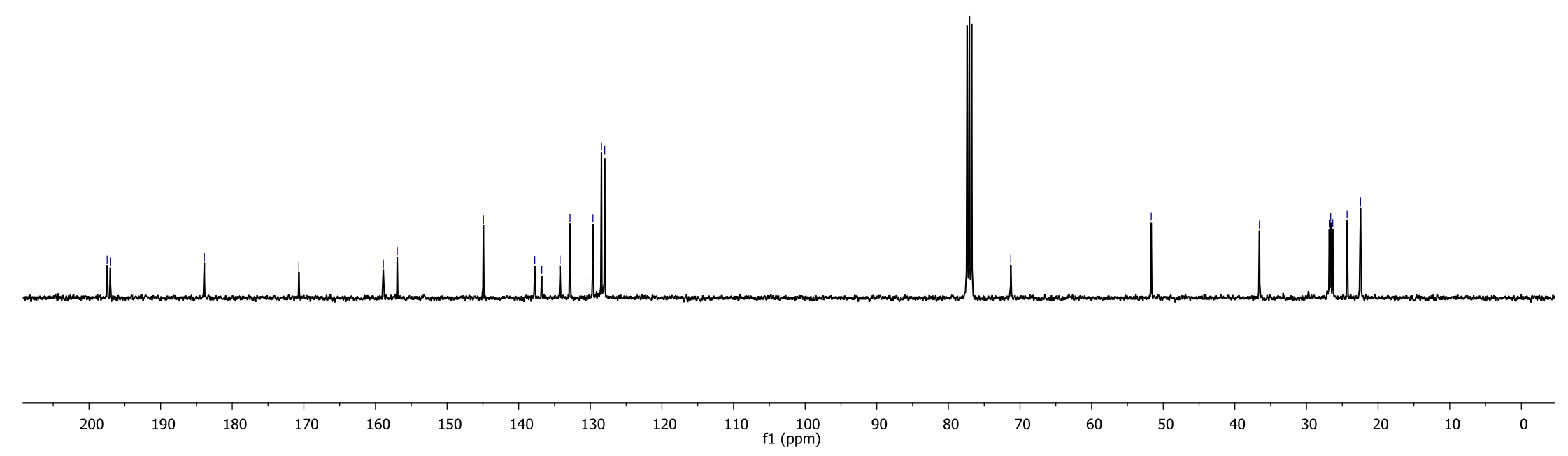


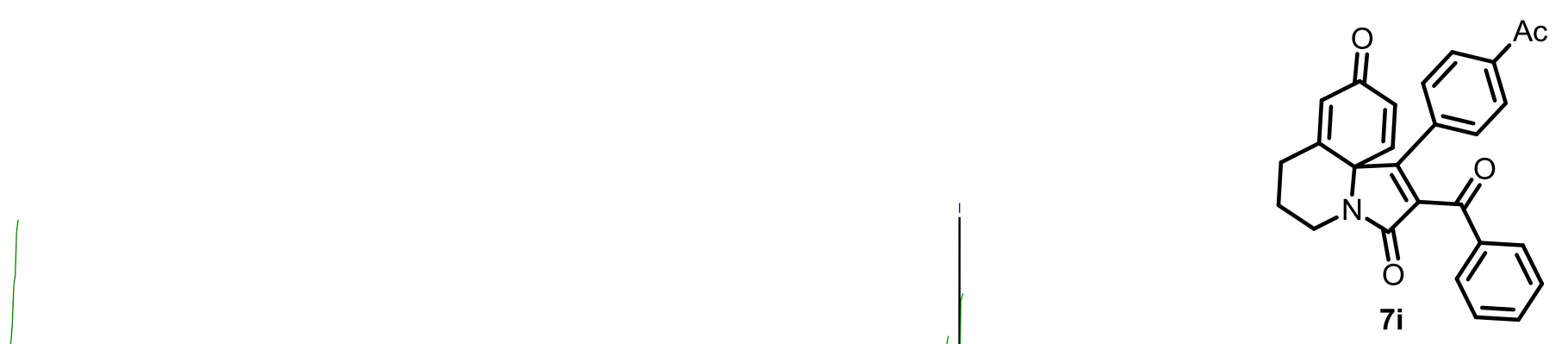

${ }^{1} \mathrm{H} \mathrm{NMR}, 500 \mathrm{MHz}, \mathrm{CDCl}_{3}$

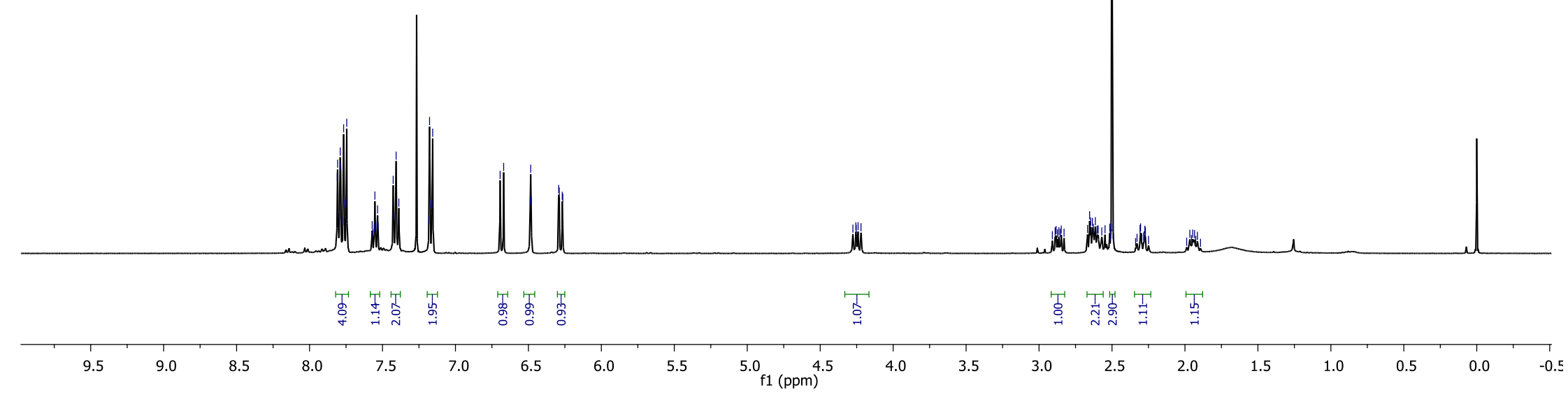




$$
\text { 1 }
$$




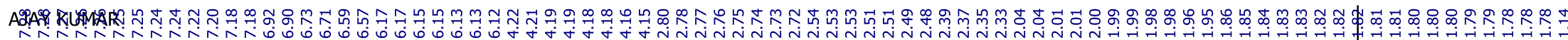<smiles>CC(C)(C)C1=C(c2ccccc2)C23C=CC(=O)C=C2CCCN3C1=O</smiles>

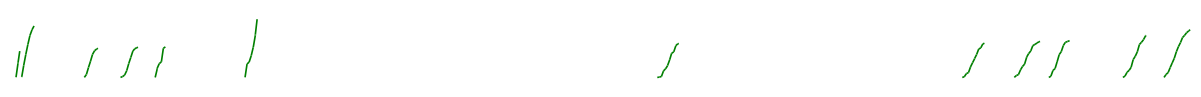

${ }^{1} \mathrm{H}$ NMR, $\mathrm{CDCl} 3,400 \mathrm{MHz}$

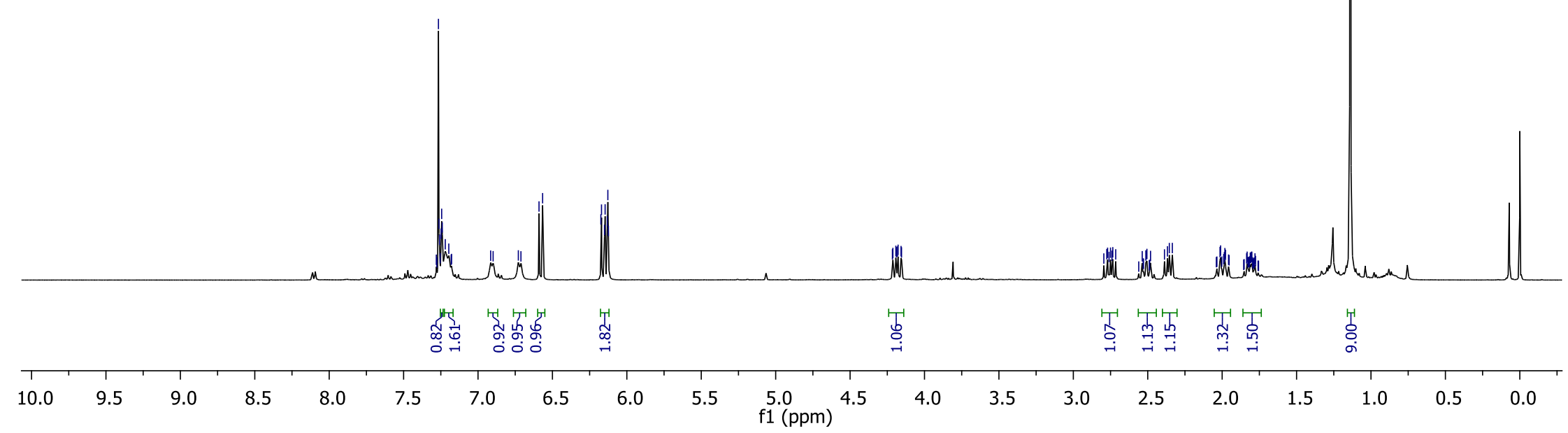


AJAY KUMAR
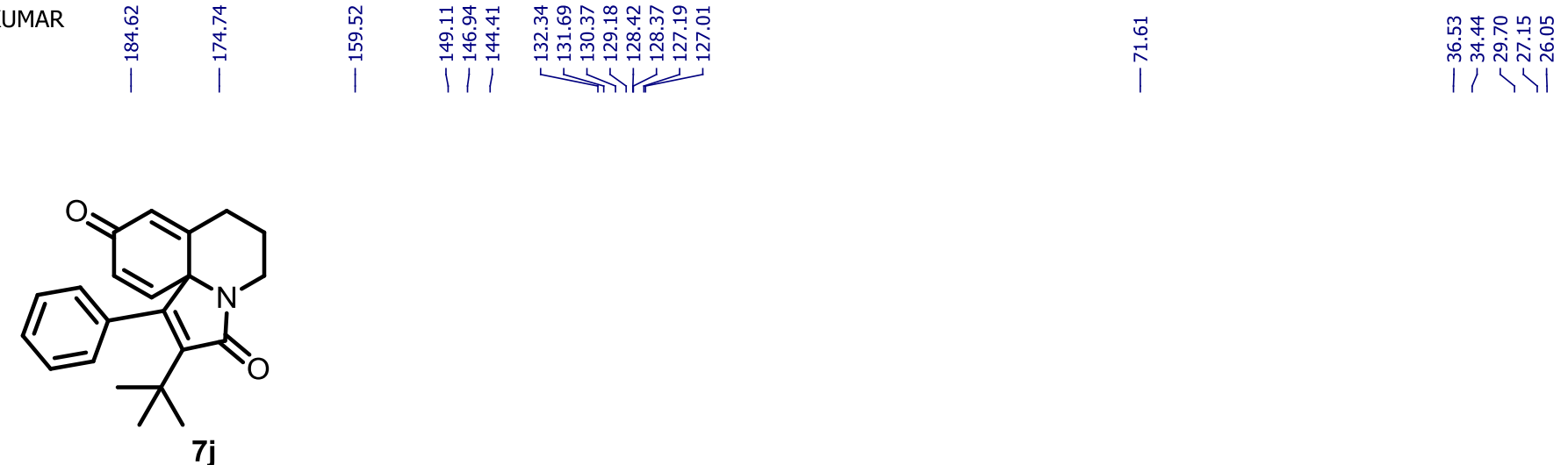

${ }^{13} \mathrm{C}$ NMR, $\mathrm{CDCI} 3,100 \mathrm{MHz}$

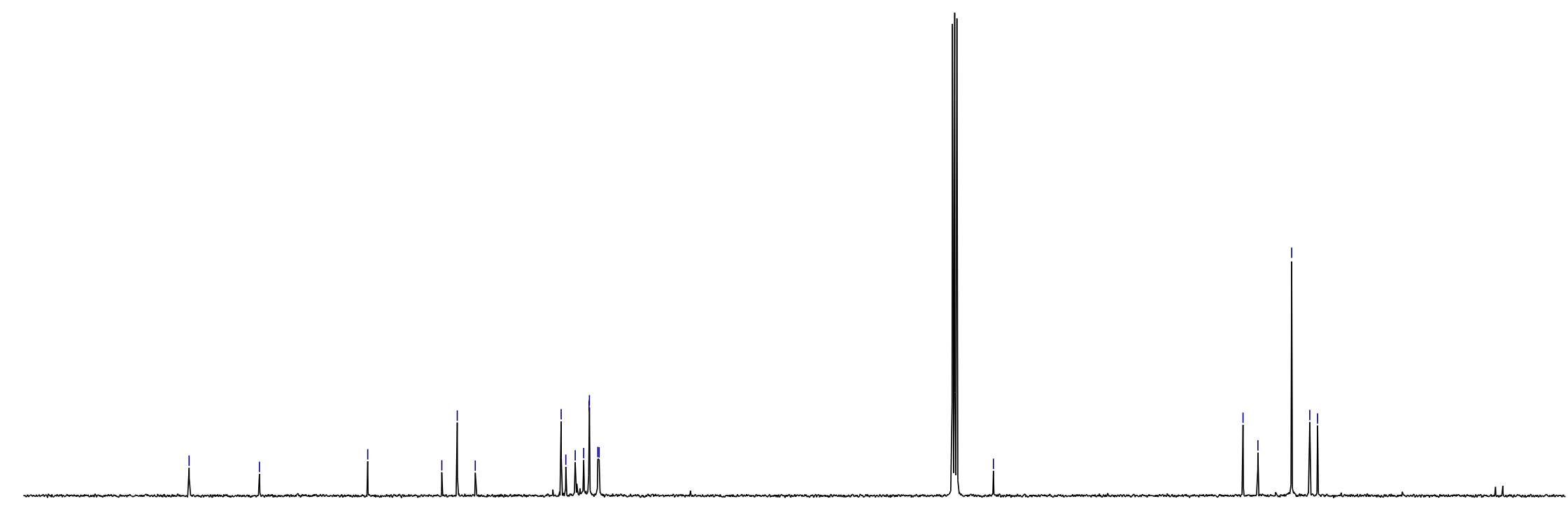

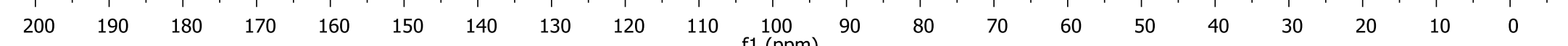




\section{HRMS spectral copy of $\mathrm{H}_{2}{ }^{18} \mathrm{O}$ experiment:}

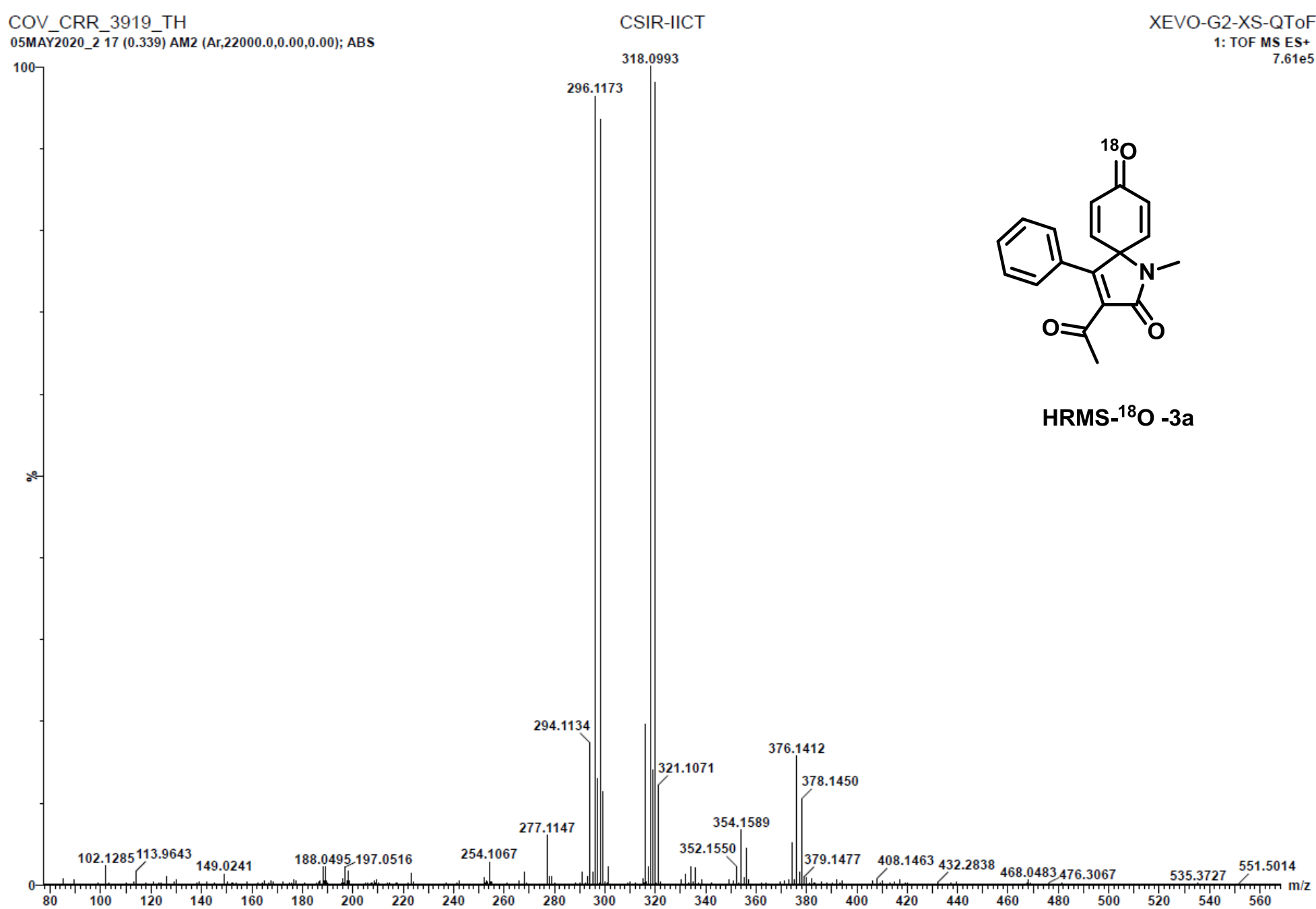




\section{Elemental Composition Report}

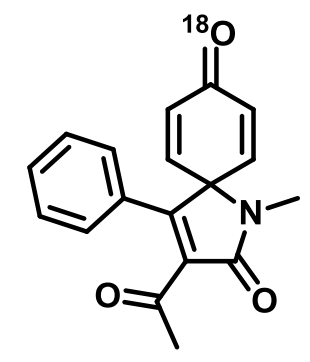

\section{Single Mass Analysis}

Tolerance $=5.0$ PPM / DBE: $\min =-1.5, \max =50.0$

Element prediction: Off

Number of isotope peaks used for $\mathrm{i}-\mathrm{FIT}=3$

HRMS $-{ }^{18} \mathrm{O}-3 \mathrm{a}$

Monoisotopic Mass, Even Electron lons

376 formula(e) evaluated with 1 results within limits (up to 50 closest results for each mass)

Elements Used:

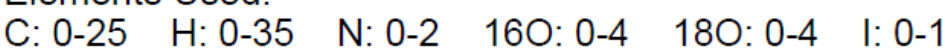

COV_CRR_3919_TH

CSIR-IICT

XEVO-G2-XS-QToF

1: TOF MS ES+

$7.61 \mathrm{e}+005$

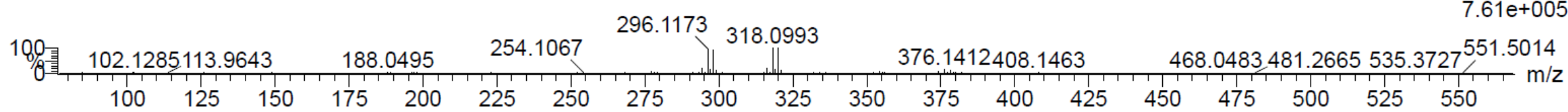

Minimum:

Maximum:

$-1.5$

Mass

Calc. Mass

5.0

5.0

50.0

296.1173

296.1173

0.0

0.0

$11.5688 .1 \mathrm{n} / \mathrm{a}$

Conf (음 Formula 
8. HRMS spectral copy Control experiment:

\section{Elemental Composition Report}

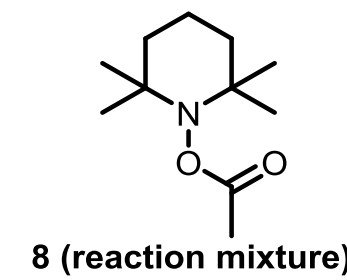

Page 1

\section{Single Mass Analysis}

Tolerance $=20.0$ PPM / DBE: $\min =-1.5, \max =50.0$

Element prediction: Off

Number of isotope peaks used for $\mathrm{I}-\mathrm{FIT}=3$

Monoisotopic Mass, Even Electron lons

16 formula(e) evaluated with 1 results within limits (up to 50 closest results for each mass)

Elements Used:

$\begin{array}{llll}\mathrm{C}: 0-12 & \mathrm{H}: 0-25 & \mathrm{~N}: 0-1 & \mathrm{O}: 0-2 \\ \mathrm{Na}: 0-1\end{array}$

CRR T $\quad$ CSIR-IICT

16JUNE2020_18 21 (0.406) AM2 (Ar,22000.0,0.00,0.00); ABS; Cm (16:33-(1:16+27:49))

XEVO-G2-XS-QTOF 1: TOF MS ES+

$3.28 \mathrm{e}+003$

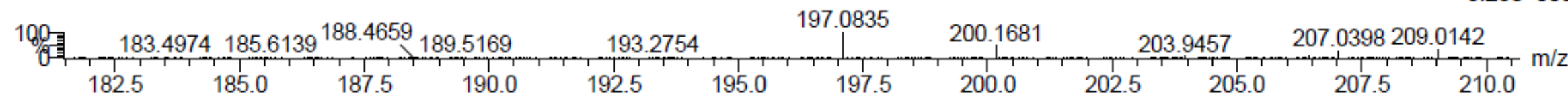

Minimum:

Maximum:

$-1.5$

Mass

$5.0 \quad 20.0 \quad 50.0$

200.1681

Calc. Mass mDa

PPM

$\mathrm{DBE}$

i-FIT

Norm

Conf (웅) Formula

200.1681

200.1651

3.0

15.0

$1.5 \quad 234.9$

$\mathrm{n} / \mathrm{a}$

$\mathrm{n} / \mathrm{a}$

C11 H22 N 02 